\title{
Homing in on consciousness in the nervous system: An action-based synthesis
}

\author{
Ezequiel Morsella \\ Department of Psychology, San Francisco State University, San Francisco, \\ CA 94132-4168; Department of Neurology, University of California, \\ San Francisco, San Francisco, CA 94158 \\ morsella@sfsu.edu \\ http://online.sfsu.edu/morsella/people.html \\ Christine A. Godwin \\ School of Psychology, Georgia Institute of Technology, Atlanta, GA 30318 \\ cgodwin9@gatech.edu \\ http://control.gatech.edu/people/graduate/cgodwin/ \\ Tiffany K. Jantz \\ Department of Psychology, University of Michigan, Ann Arbor, MI 48109-1043 \\ tkjantz@umich.edu \\ http://prod.Isa.umich.edu/psych/people/graduate-students/tkjantz.html
}

\author{
Stephen C. Krieger \\ Department of Neurology, Mount Sinai Medical Center, New York, \\ NY 10029-6574 \\ stephen.krieger@mssm.edu \\ http://www.mountsinai.org/profiles/stephen-krieger
}

\section{Adam Gazzaley}

Department of Neurology, Department of Psychiatry, and Department of Physiology, University of California, San Francisco, San Francisco, CA, 94158 adam.gazzaley@ucsf.edu

http://gazzaleylab.ucsf.edu/people-profiles/adam-gazzaley/

\begin{abstract}
What is the primary function of consciousness in the nervous system? The answer to this question remains enigmatic, not so much because of a lack of relevant data, but because of the lack of a conceptual framework with which to interpret the data. To this end, we have developed Passive Frame Theory, an internally coherent framework that, from an action-based perspective, synthesizes empirically supported hypotheses from diverse fields of investigation. The theory proposes that the primary function of consciousness is well-circumscribed, serving the somatic nervous system. For this system, consciousness serves as a frame that constrains and directs skeletal muscle output, thereby yielding adaptive behavior. The mechanism by which consciousness achieves this is more counterintuitive, passive, and "low level" than the kinds of functions that theorists have previously attributed to consciousness. Passive frame theory begins to illuminate (a) what consciousness contributes to nervous function, (b) how consciousness achieves this function, and (c) the neuroanatomical substrates of conscious processes. Our untraditional, action-based perspective focuses on olfaction instead of on vision and is descriptive (describing the products of nature as they evolved to be) rather than normative (construing processes in terms of how they should function). Passive frame theory begins to isolate the neuroanatomical, cognitivemechanistic, and representational (e.g., conscious contents) processes associated with consciousness.
\end{abstract}

Keywords: Consciousness; neural correlates of consciousness; subjective experience; unconscious processing; voluntary action

What does consciousness contribute to the functioning of the nervous system? What is the primary role of this elusive phenomenon? The answers to these questions remain enigmatic, not so much because of a lack of relevant data, but because of the lack of a conceptual, internally coherent framework with which to interpret the data
(Grossberg 1987). Hence, we developed Passive Frame Theory, a framework that, from an action-based perspective, synthesizes empirically supported hypotheses from diverse fields of investigation. The framework begins to illuminate (a) what consciousness contributes to nervous function, (b) how consciousness achieves this function, 
Morsella et al.: Homing in on consciousness in the nervous system

and (c) the neuroanatomical substrates of conscious processes. Passive frame theory proposes that the primary function of consciousness is well circumscribed, serving the somatic nervous system. For this system, it serves as a frame that constrains and directs skeletal muscle output, thereby yielding adaptive behavior. The mechanism by which consciousness achieves this is more counterintuitive,

EzEquiel Morsella is a theoretician and experimentalist who has devoted his entire career to investigating the differences in the brain between the conscious and unconscious circuits in the control of human action. He is the lead author of Oxford Handbook of Human Action (2009, Oxford University Press) and was the editor of the Festschrift in honor of Robert M. Krauss. His research has appeared in leading journals in neuroscience and experimental psychology, including Psychological Review, Neurocase, and Consciousness and Cognition. After his undergraduate studies at the University of Miami, he carried out his doctoral and postdoctoral studies at Columbia University and Yale University, respectively.

Christine A. Godwin is currently a Ph.D. student in the School of Psychology at the Georgia Institute of Technology. She received her Master of Arts in Psychological Research from San Francisco State University, where she co-authored several publications in the area of action and consciousness. Her current work focuses on the temporal dynamics of memory encoding processes and cognitive control.

Tiffany K. Jantz is currently a Ph.D. student of Psychology with an emphasis in cognition and cognitive neuroscience at the University of Michigan. She received her Master of Arts in Psychological Research from San Francisco State University, where she was awarded the Graduate Student Award for Distinguished Achievement in Psychology. She has published in the area of cognition, including consciousness, imagery, working memory, attention, and cognitive aging.

Stephen C. Krieger, MD, is an Associate Professor of Neurology at the Icahn School of Medicine at Mount Sinai, where he is the Director of the Neurology Residency Program and a specialist in multiple sclerosis. A noted neurology educator, he has won numerous mentorship and teaching awards at Mount Sinai, and received an American Academy of Neurology A. B. Baker teaching recognition award in 2010. His research has emphasized examining diagnosis and misdiagnosis in multiple sclerosis specifically, and clinical neurology more broadly.

Adam Gazzaley, MD, Ph.D., is Professor in Neurology, Physiology, and Psychiatry at the University of California, San Francisco, the founding director of the Neuroscience Imaging Center, and director of the Gazzaley Lab. His laboratory's most recent studies explore neuroplasticity and how we can optimize our cognitive abilities via engagement with custom-designed video games, as well as how this can be bolstered by closedloop systems using neurofeedback and transcranial electrical stimulation. He has filed multiple patents based on his research, authored more than 100 scientific articles, and delivered more than 425 invited presentations around the world. passive, and "low level" than the kinds of functions that theorists have attributed to consciousness.

Our unique perspective and conclusions provide a comprehensive approach to the enigma of the primary function of consciousness. To solve this puzzle, an overarching coherent framework is a necessary first step to the development of more concrete advances (e.g., hypotheses for the derivation of experiment-specific predictions). Before discussing the hypotheses that serve as the tenets of passive frame theory, it is necessary to define some terms and describe the nature of our untraditional approach.

\section{Purview, terms, and assumptions}

\subsection{The scientific approach should focus on the most basic form of consciousness}

We believe that, to advance the study of consciousness, one should focus not on high forms of consciousness (e.g., "selfconsciousness"), but on the most basic forms of consciousness (e.g., the experience of a smell, visual afterimages, tooth pain, or urges to scratch an itch). This form of consciousness has fallen under the rubrics of "sentience" (Pinker 1997), "primary consciousness" (Edelman 1989), "phenomenal consciousness" (Block 1995b), "qualia” (J. A. Gray 2004), "phenomenal states" (Tye 1999), and "subjective experience." In our framework, we refer to a thing of which one is conscious (e.g., an afterimage) as a conscious content (Merker 2007; Seth 2007). All of the contents of which one is conscious at one time can be construed as composing the conscious field (Freeman 2004; Köhler 1947; Searle 2000). The contents of the conscious field change over time.

\subsection{The approach should be descriptive, non-normative}

We believe that the approach to consciousness should be a descriptive, naturalistically based one (which describes the products of nature as they evolved to be) rather than a normative one (which construes processes in terms of how they should function). Nervous mechanisms have been fashioned by the happenstance and tinkering process of evolution, whose products can be counterintuitive and suboptimal (de Waal 2002; Gould 1977; Lorenz 1963; Marcus 2008; Roe \& Simpson 1958; Simpson 1949), far unlike the kinds of things humans design into machines (Arkin 1998). Hence, the ethologist Konrad Lorenz (1963) cautions, "To the biologist who knows the ways in which selection works and who is also aware of its limitations it is in no way surprising to find, in its constructions, some details which are unnecessary or even detrimental to survival" (p. 260). Similarly, when speaking about the reverse engineering of biological products, the roboticist Ronald Arkin concludes, "Biological systems bring a large amount of evolutionary baggage unnecessary to support intelligent behavior in their silicon based counterparts" (Arkin 1998, p. 32). The difference between the products of evolution and human artifacts is obvious when one considers the stark contrast between human locomotion and artificial locomotion - legs versus wheels (Morsella \& Poehlman 2013).

When adopting a descriptive standpoint, even the most cursory examination of the brain reveals a contrast between conscious and unconscious processes (see Bleuler 1924). Thus, in every field of inquiry, there is the de facto distinction between the two kinds of processes, 
Morsella et al.: Homing in on consciousness in the nervous system

though often without mention of the taboo term "consciousness." For example, in perception research, there exists the distinction between supra-versus subliminal. In memory research, there is the distinction between "declarative" (explicit) processes and "procedural" (implicit) processes (Schacter 1996; Squire 1987). In motor and language research, the conscious aspects of voluntary action or of speech production are contrasted with the unconscious aspects of, say, motor programming (Levelt 1989; Rosenbaum 2002; J. A. Taylor \& Ivry 2013). Various fields also contrast "controlled" processing (which tends to be conscious) and "automatic" processing (which is often unconscious; Lieberman 2007). In summary, from a descriptive approach, the contrast between conscious and unconscious processes in the brain is somewhat inevitable (Morsella \& Poehlman 2013).

\subsection{The approach should be minimalistic, focusing on simple cases}

When attempting to unravel a phenomenon as perplexing as consciousness, it is important to adopt a strategy in which scientific inquiry begins with the examination of the most basic, elemental instantiation of the phenomenon of interest (Panksepp 2007). Such a strategy proved fruitful in the development of physics (Einstein \& Infeld 1938/ 1967). Hence, in our approach, we focus on the actions of a hypothetical, simplified, human-like mammal that, though conscious (for a treatment of consciousness in mammals, see J. A. Gray [2004]), is not equipped with many of the complicated abilities/states associated with adult humans (e.g., syntax and music appreciation). Capable of having approach-avoidance conflicts (Lewin 1935; N. E. Miller 1959), this hypothetical organism is occupied only with basic operations (e.g., breathing, locomoting, and avoiding tissue damage) rather than with higher-level phenomena (e.g., mental rotation and sustained, directed thinking). This organism is also incapable of indirect cognitive control (Morsella et al. 2009b), whereby one can, for instance, make oneself hungry or scared by deliberately imagining the kinds of things that would induce these states. Such higher-level phenomena are more likely to be predicated upon (a) extensive learning, (b) cultural influences, (c) intricate interactions among more elemental conscious processes, and (d) adaptations that are less phylogenetically primitive than those of the basic operations of interest (Morsella 2005).

In our "simple case," this hypothetical organism is resting in a warm enclosure (e.g., a cave). It can consciously perceive an opening from which it could exit. For hours, the organism performs no notable locomotive act toward the opening nor to anything else, but then it perceives a noxious smell (e.g., smoke) from within the enclosure. Because of this new conscious content, it now exits hesitantly through the opening, even though it was inclined to remain within the warm enclosure. To illuminate the nature of consciousness, we will revisit this "creature in the cave" scenario throughout our treatise. We refer to the first events composing the scenario (e.g., the percept of the opening and the warmth) as Stage 1, and the subsequent events (e.g., the smell and the inclination to stay in the cave) as Stage 2.

In contrast to our strategy, descriptive accounts of consciousness have tended to focus on high-level functions, leading to conclusions such as that the function of consciousness pertains to social interaction (Frith 2010; Prinz 2012), language (Banks 1995; Carlson 1994; Macphail 1998), "theory of mind" (Stuss \& Anderson 2004), the formation of the self (Greenwald \& Pratkanis 1984), semantic processing (Kouider \& Dupoux 2004; Mudrik et al. 2014; Thagard \& Stewart 2014), the meaningful interpretation of situations (Roser \& Gazzaniga 2004), and simulations of behavior and perception (Hesslow 2002). (It is worth noting that, for good reasons, it has also been proposed that, contrary to the present account, consciousness does not contribute to ongoing action; see Hommel 2013; Jackson 1986; Kinsbourne 1996; 2000; Masicampo \& Baumeister 2013; Pinker 1997.)

\subsection{Overview of present, untraditional approach}

Our approach is untraditional in several ways. First, instead of focusing on the relationship between consciousness and perception (which has been the dominant approach; Crick \& Koch 2003; Rosenbaum 2005), we focus on the relationship between consciousness and overt action. Second, unlike traditional stimulus $\rightarrow$ response approaches, we "work backward" from overt action to the underlying processes responsible for it (Sperry 1952). Thus, from our untraditional, action-based approach, we subscribe to an uncommon theoretical position - that the nature of consciousness is best understood by examining the requirements of adaptive (efferent) action control rather than the needs of perceptual analysis. From this unconventional approach to consciousness, one can appreciate that the requirements of adaptive skeletomotor action reveal much about the nature of both the conscious field and the generation of conscious contents. Third, building on Morsella and Bargh (2007), instead of focusing on vision to understand consciousness (which has been the prevalent approach; Crick \& Koch 2003), we focus on the (possibly) more tractable system of olfaction, as illustrated in our "creature in the cave" example. The olfactory system possesses several features that render it a fruitful system in which to study consciousness. ${ }^{1}$

To summarize, our approach is elemental, action-based, simple, and evolutionary-based (or, for short, "EASE," meaning "to make something less difficult"). We believe that an EASE perspective provides the most fruitful approach to the perplexing problem of consciousness and the brain. Whenever in our enterprise we encountered an obstacle for theoretical progress (e.g., the neural regions associated with consciousness), it was through our EASE perspective that progress was made. In the next three sections, we discuss from an EASE perspective the empirically supported hypotheses that serve as the tenets of passive frame theory. Through the process, we begin to isolate the neuroanatomical, cognitive-mechanistic, and representational (e.g., conscious contents; sect. 3) processes associated with consciousness.

\section{The circumscribed role of consciousness in the nervous system}

\subsection{Tenet: Consciousness is associated with only a subset of nervous function}

Based on developments of the past four decades, there is a growing subset consensus - that consciousness is associated with only a subset of all of the processes and regions of the 
Morsella et al.: Homing in on consciousness in the nervous system

nervous system ${ }^{2}$ (Aru et al. 2012; Crick \& Koch 1995; 2003; Dehaene \& Naccache 2001; J. A. Gray 2004; Grossberg 1999; Koch 2004; Koch \& Greenfield 2007; Logothetis \& Schall 1989; Merker 2007; 2013c; Penfield \& Jasper 1954; Weiskrantz 1992; Zeki \& Bartels 1999). This subset seems to be qualitatively distinct - in terms of its functioning, physical makeup/organization, or mode of activity from that of its unconscious counterparts in the brain (Bleuler 1924; Coenen 1998; Edelman \& Tononi 2000; Goodale \& Milner 2004; J. A. Gray 2004; Llinás et al. 1998; Merker 2007; Ojemann 1986).

Consistent with the subset consensus, many aspects of nervous function are unconscious. ${ }^{3}$ Complex processes of an unconscious nature can be found at all stages of processing (Velmans 1991), including low-level perceptual analysis (e.g., motion detection, color detection, auditory analysis; Zeki \& Bartels 1999), semantic-conceptual processing (Harley 1993; Lucas 2000), and motor programming (discussed in sect. 3.1). Evidence for the complexity of unconscious processing is found in cases in which the entire stimulus-response arc is mediated unconsciously, as in the case of unconsciously mediated actions (e.g., automatisms). There is a plethora of evidence that action plans can be activated, selected, and even expressed unconsciously. ${ }^{4}$ In summary, it seems that much in the nervous system is achieved unconsciously. This insight from the subset consensus leads one to the following question: What does consciousness contribute to nervous function?

\subsection{Tenet: The conscious field serves an integrative role}

The integration consensus (Baars 1988; 1998; 2002; 2005; Boly et al. 2011; Clark 2002; Damasio 1989; Dehaene \& Naccache 2001; Del Cul et al. 2007; Doesburg et al. 2009; Edelman \& Tononi 2000; Freeman 1991; Koch 2012; Kriegel 2007; Llinás \& Ribary 2001; Merker 2007; Ortinski \& Meador 2004; Sergent \& Dehaene 2004; Srinivasan et al. 1999; Tallon-Baudry 2012; Tononi 2012; Tononi \& Edelman 1998; Uhlhaas et al. 2009; Varela et al. 2001; Zeki \& Bartels 1999) proposes that consciousness integrates neural activities and information-processing structures that would otherwise be independent. Most of the hypotheses comprising this consensus speak of conscious information as being available "globally," in some kind of workspace, as in Baars's (1988) influential global workspace theory. For present purposes, we construe the contents occupying such a workspace as composing the conscious field (defined in sect. 1.1 above).

Consistent with the integration consensus, the conscious processing of a percept involves a wider and more diverse network of regions than does the subliminal (unconscious) processing of the same percept (Singer 2011; Uhlhaas et al. 2009). The latter is subjected only to "local" processing. This evidence stemmed initially from research on perception (Del Cul et al. 2007; Uhlhaas et al. 2009), anesthesia (Alkire et al. 2008; Boveroux et al. 2010; Långsjö et al. 2012; Lee et al. 2009; Lewis et al. 2012; Schroter et al. 2012; Schrouff et al. 2011), and unresponsive states (e.g., coma or vegetative state; Laureys 2005). Regarding perception research, it has been proposed that, during binocular rivalry, ${ }^{5}$ the neural processing of the conscious percept requires special interactions between both perceptual regions and other, traditionally non-perceptual regions (e.g., frontal cortex; Doesburg et al. 2009). This supports the view that some mode of interaction between widespread brain areas is important for consciousness (Buzsáki 2006; Doesburg et al. 2009; Fries 2005; Hummel \& Gerloff 2005).

Evidence for the integration consensus is found also in action-based research. Conscious actions involve more widespread activations in the brain than do similar but unconscious actions (Kern et al. 2001; McKay et al. 2003; Ortinski \& Meador 2004). Moreover, when actions are decoupled from consciousness (e.g., in neurological disorders), the actions often appear impulsive or inappropriate, as if they are not adequately influenced by the kinds of information by which they should be influenced (Morsella \& Bargh 2011).

\subsection{Advances regarding the physiological processes engendering consciousness depend on advances regarding the neuroanatomy of consciousness}

The nature of the neuroanatomical network engendering the physiological processes (e.g., neural oscillations) proposed to be associated with consciousness remains controversial. $^{6}$ Progress regarding the neurophysiology of consciousness depends on advances regarding the identification of the neuroanatomical substrates of this state (Aru et al. 2012). Regarding neuroanatomy, when attempting to isolate the anatomical underpinnings of consciousness, investigators have followed Crick and Koch's (2003) recommendation and have focused on vision. (See reviews of neural correlates of visual consciousness in Blake and Logothetis [2002], Dehaene [2014], Koch [2004], Lamme and Spekreijse [2000], Metzinger [2000], and Tong [2003].) In vision research, controversy remains regarding whether consciousness depends on higher-order perceptual regions (Crick \& Koch 1995; 1998; Panagiotaropoulos et al. 2012; 2013) or lower-order regions (Aru et al. 2012; Damasio, 2010; Friedman-Hill et al. 1995; Lamme 2001; Liu et al. 2012; Robertson 2003; Tallon-Baudry 2012; Tong 2003). Moreover, as noted in Note 2, whether cortical matter is necessary for consciousness remains controversial.

Theorists focusing on vision have proposed that, although the cortex may elaborate the contents of consciousness, consciousness is primarily a function of subcortical structures (Merker 2007; Penfield \& Jasper 1954; Ward 2011). Penfield and Jasper (1954) based this hypothesis on their studies involving both the direct stimulation of, and ablation of, cortical regions. Based on these and other findings (e.g., observations of patients with anencephaly; Merker 2007), it has been proposed that consciousness is associated with subcortical areas (e.g., Merker 2007; 2013c). This has led to the cortical-subcortical controversy (Morsella et al. 2011). While data from studies on patients with profound disorders of consciousness (e.g., vegetative state) suggest that signals from the frontal cortex may be critical for the instantiation of any form of consciousness (Boly et al. 2011; Dehaene \& Naccache 2001; Lau 2008; Panagiotaropoulos et al. 2012; Velly et al. 2007), research on the psychophysiology of dream consciousness, which involves prefrontal deactivations (Muzur et al. 2002), suggests that, although the prefrontal lobes are involved in cognitive control, they may not be essential for the generation of basic consciousness (Aru et al. 2012; Merker 2007; Ward 2011). Regarding the necessity of the integrity of the frontal lobes for consciousness, it is 
Morsella et al.: Homing in on consciousness in the nervous system

important to consider that the surgical procedure of frontal lobotomy, once a common neurosurgical intervention for the treatment of psychiatric disorders, was never reported to render patients incapable of sustaining consciousness (see also Aleman \& Merker 2014).

The role of subcortical structures in the production of consciousness, and the amount of cortex that may be necessary for the production of consciousness, remains to be elucidated (see further discussion in sect. 3.5). Clearly, more investigation is needed regarding the neural correlates of consciousness, because controversy continues to surround not only the neurophysiological processes underlying consciousness, but even the identification of the gross, neuroanatomical regions that are responsible for this peculiar form of processing (see treatment in Merker 2007; 2013b).

Faced with this challenge, we propose that, because of the intimate liaison between function and structure in the nervous system (Cohen \& Dennett 2011; Merker 2013c), progress can be made regarding the neural underpinnings of consciousness by having a more precise understanding of the role of consciousness in nervous function (Lamme \& Spekreijse 2000). With this in mind, one can reason as follows: If the consensus is that consciousness serves an integrative role, then, from an EASE perspective, what is the most basic form of integration that requires consciousness? Addressing this question allows one to better isolate consciousness within the nervous system, which could, in turn, resolve controversies regarding the neural correlates of consciousness.

\subsection{Tenet: The conscious field is for a specific kind of integration, involving the skeletal muscle output system}

One limitation of the integration consensus is that it fails to specify which kinds of integrations require consciousness and which kinds do not. Consciousness seems unnecessary for various kinds of integrations in the nervous system. For example, integrations across different sensory modalities, as in the case of afference binding (Morsella \& Bargh 2011), can occur unconsciously. This form of integration occurs in feature binding (e.g., the binding of shape to color; Zeki \& Bartels 1999) and in intersensory binding (Vroomen \& de Gelder 2003), as in the ventriloquism and McGurk effects (McGurk \& MacDonald 1976). The latter, for instance, involves interactions between visual and auditory processes: An observer views a speaker mouthing "ga" while presented with the sound "ba." Surprisingly, the observer is unaware of any intersensory interaction, perceiving only "da." (See list of many kinds of unconscious afference binding in Morsella [2005], Appendix A.) Integrations involving smooth muscle effectors (e.g., in peristalsis or in the pupillary reflex), too, can occur unconsciously (Morsella et al. 2009a), as can another form of integration known as efference binding (Haggard et al. 2002).

Efference binding links perceptual processing to action/ motor processing. This kind of stimulus-response binding is mediated unconsciously in actions such as reflexive pain withdrawal or reflexive inhalation. In learned behavior, efference binding allows one to press a button when presented with an arbitrary cue. Such a form of binding can be learned quickly (e.g., from a few trials of stimulus- response mapping; Hommel \& Elsner 2009) and with little effort (Cohen-Kdoshay \& Meiran 2009; Melcher et al. 2008). Learned forms of efference binding can be expressed unconsciously (Fehrer \& Biederman 1962; Fehrer \& Raab 1962; Hallett 2007; J. L. Taylor \& McCloskey 1990; 1996). For example, subjects can select the correct motor response (one of two button presses) when confronted with subliminal stimuli, suggesting that "appropriate programs for two separate movements can be simultaneously held ready for use, and that either one can be executed when triggered by specific stimuli without subjective awareness" (Taylor \& McCloskey 1996, p. 62; see review in Hallett 2007). We return to the topic of efference binding when discussing how conscious contents influence action (sect. 3.2).

In contrast to these unconscious forms of integration, people tend to be very much aware of some integrations, as when one holds one's breath while underwater or experiences an approach-avoidance conflict (Lewin 1935; N. E. Miller 1959). In the former, one experiences the inclinations to both inhale and to not inhale. Similarly, when carrying a hot dish of food, one experiences the inclinations to drop the dish and to not drop the dish (Morsella 2005). Unlike unconscious integrations, such conscious conflicts (Morsella 2005) reflect a form of integration that is associated not with perceptual processing, but rather with action selection. ${ }^{7}$ This form of integration has been distinguished from unconscious integrations/conflicts, such as the McGurk effect and smooth muscle conflicts (e.g., in the pupillary reflex). In short, conflicts at the stage of processing of action selection are experienced consciously, whereas conflicts at perceptual stages of processing are unconscious. It has been proposed that, unlike unconscious integrations, these integrations involve competition for control of the skeletal muscle ("skeletomotor," for short) output system (Morsella 2005). The skeletomotor output system contains the unconscious motor plans that are necessary to enact one skeletomotor act versus another (Bizzi \& MussaIvaldi 2004; Rizzolatti et al. 2004; Rosenbaum 2002). It stores, for example, the unconscious articulatory plans that are necessary for speech production (Buchsbaum 2013) and the plans for blinking (Graziano 2008). When these plans are stimulated sufficiently, overt actions arise.

Involving urges and other action-related inclinations, conscious conflicts occur when two streams of efference binding are trying to influence skeletomotor action simultaneously (Morsella \& Bargh 2011). For example, conscious conflicts occur when one holds one's breath, suppresses uttering something, suppresses a prepotent response in a response interference paradigm, or voluntarily breathes faster for some reward. (The last example illustrates that not all cases of this kind of integration involve suppression.) These conscious conflicts appear to be triggered into existence by the activation of incompatible skeletomotor plans. ${ }^{8}$ In our "creature in the cave" scenario, this form of integration occurs when the organism is inclined to both exit the enclosure (because of the smoke) and remain within it (because of the warmth).

Thus, Morsella (2005) proposes that the primary function of consciousness is to integrate information, but only certain kinds of information - the kinds involving incompatible skeletal muscle intentions for adaptive action (e.g., holding one's breath while underwater). ${ }^{\text {T }}$ From this standpoint, the conscious field is unnecessary to integrate 
Morsella et al.: Homing in on consciousness in the nervous system

perceptual-level processes (as in feature binding or intersensory conflicts), smooth muscle processes (e.g., pupillary reflex; Morsella et al. 2009a), or processes associated with motor control (discussed in sect. 3.1 below). Instead, the conscious field is necessary to integrate what appear to be multiple inclinations toward the skeletomotor output system, as captured by the principle of Parallel Responses into Skeletal Muscle (PRISM; Morsella 2005). From this perspective, and as fleshed out in the next section, it is this third kind of binding that is the most basic form of integration that requires consciousness. PRISM explains why, phenomenologically, a wink is different from a reflexive blink and from the dilation of a pupil.

\subsection{Tenet: The conscious field is for adaptive voluntary action}

In colloquial terms, one can conclude that consciousness is for adaptive "voluntary" action. Scientifically, consciousness can be construed as the medium that allows action processes to influence skeletomotor action collectively, leading to integrated actions (Morsella \& Bargh 2011), such as holding one's breath. Just as a prism combines different colors to yield a single hue, the conscious field permits for multiple response tendencies to yield a single, integrated action. Absent consciousness, skeletomotor behavior can be influenced by only one of the efference streams, leading to unintegrated actions (Morsella \& Bargh 2011), such as unconsciously inhaling while underwater or reflexively removing one's hand from a hot object. Reflecting a lack of integration, unintegrated actions appear as if they are not influenced by all of the kinds of information by which they should be influenced. If a conscious content is not in the field, then it cannot influence voluntary action. For example, if the knowledge representations necessary for, say, "reality monitoring," are not in the field (e.g., due to fever), then nothing else can assume the functional influence of these contents. (This is evident in action selection in dreams, which are often irrational, and in disorders of awareness, such as sensory neglect and anosognosia.) Therefore, in voluntary action, when the appropriate contents are absent, there is no independent system or repository of knowledge that can step in to fill their role. Thus, the conscious field wholly and exclusively determines what in everyday life is called voluntary behavior. Conversely, for every voluntary action, the organism can report a conscious content responsible for that action, regardless of the veracity of the introspection (Poehlman et al. 2012).

These conclusions also reveal that it is no accident that, historically, skeletal muscle has been described as "voluntary" muscle. Since at least the nineteenth century, it has been known that, though often functioning unconsciously (as in the frequent actions of breathing and blinking), skeletal muscle is the only bodily effector that can be consciously controlled, but why this is so has never been addressed theoretically. PRISM introduces a systematic reinterpretation of this age-old fact (Morsella 2005): Skeletomotor actions are at times "consciously mediated" because they are directed by multiple systems that require consciousness to influence action collectively - what we refer to as collective influence.

Regarding the skeletomotor output system, one must consider that all processes trying to influence skeletomotor behavior must, in a sense, "go through it." Each system giving rise to inclinations has its peculiar operating principles and phylogenetic origins (Allman 2000): One system "protests" an exploratory act while another system reinforces that act (Morsella 2005). Because each skeletomotor effector can usually perform only one act at a time (e.g., one can utter only one word at a time; Lashley 1951; Wundt 1900), there must be a way in which the inclinations from the many heterogeneous systems can be "understood" and processed collectively by the skeletomotor output system. To yield adaptive action, this process must also integrate information about other things (e.g., the physical environment). To a degree greater than that of any other effector system (e.g., smooth muscle), distinct regions/ systems of the brain are trying to control the skeletomotor output system in different and often opposing ways. All inclinations toward it, from primitive plans about basic needs to complex plans associated with language, must engage this system. Thus, the skeletomotor output system is the "final common path" for processes capable of influencing skeletomotor function (McFarland \& Sibly 1975; Sherrington 1906). Figuratively speaking, the skeletomotor output system is akin to a single steering wheel that is controlled by multiple drivers (Morsella 2005).

\section{Conscious contents}

If one accepts that consciousness is in the service of voluntary action, then, from an EASE perspective and by working backward from overt action to central processing, one can ask the following question: What kinds of information and knowledge representation (Markman 1999) render voluntary action adaptive? To answer this question, one must examine the nature of conscious contents while appreciating that the varied inputs to the skeletomotor output system must (a) represent information that is essential for adaptive skeletomotor action and (b) be formatted in a manner that is understandable by the unconscious, action-related mechanisms composing the skeletomotor output system (Morsella \& Bargh 2010b).

The three tenets presented in sections 3.1 through 3.3 below suggest that our "creature in the cave" is conscious of things such as external objects and the urge to eat or to affiliate (or to do other things that reflect the inclinations of the many "hot" affective/incentive systems; Metcalfe \& Mischel 1999), because these things should influence the skeletomotor output system. For this creature, it is clear that additions ${ }^{10}$ of conscious content are usually about the world, the body, or action-related inclinations (Brentano 1874; Chomsky 1988; Fodor 1980; 1998; J. A. Gray 1995; 2004; Hume 1739/1888; Koch 2004; Schopenhauer 1818/1819; Sergent \& Dehaene 2004).

\subsection{Tenet: Conscious contents must be "perceptual-like" in nature}

We propose that the cognitive and neural processes associated with the contents of our "creature in the cave" should be perceptual-like in nature. When making this claim, we acknowledge that conscious contents are neither purely sensorial nor purely motor related; instead, they represent well-crafted representations occurring at a stage of processing between sensory analysis and motor programming 
(Jackendoff 1990; Lashley 1956; Merker 2013c; J. Prinz 2007; W. Prinz 2003b). In everyday life, when speaking about this level of representation of external objects, we use the term percept (J. A. Gray 1995); however, this level of representation is more precisely construed as an intermediate representational format (e.g., the color red or the illusion of "da" in the McGurk effect) that links perception to action (W. Prinz 2003b). (To not introduce more jargon, we will continue to use the term percept to refer to conscious contents about the external world or the body, but we do so mindful that the term, because of its sensory connotation, can be misleading.)

The proposal that contents are perceptual-like is based on the synthesis of conclusions from diverse areas of study. First, according to the age-old sensorium hypothesis (Godwin et al. 2013; Goodale \& Milner 2004; J. A. Gray 2004; Grossberg 1999; Harleß 1861; James 1890; Müller 1843; Woodworth 1915), the contents of consciousness are influenced primarily by perceptual-based (and not motor-based) events and processes, because motor processes are largely unconscious. There is substantial phenomenological evidence for this hypothesis. During action, for example, one is unconscious of the efference to the muscles that dictates which fibers should be activated at which time (Rosenbaum 2002). Although one is unconscious of these complex programs (Johnson \& Haggard $2005)$, one $i s$ often aware of their proprioceptive and perceptual consequences (e.g., perceiving the hand grasping; Fecteau et al. 2001; Fourneret \& Jeannerod 1998; Gottlieb \& Mazzoni 2004; J. A. Gray 2004; Heath et al. 2008; Liu et al. 2008; Rossetti 2001). These images tend to be perceptual-like images of action outcomes (Hommel 2009; Jeannerod 2006; Pacherie 2008): "In perfectly simple voluntary acts there is nothing else in the mind but the kinesthetic idea ... of what the act is to be" (James 1890, p. 771). ${ }^{11}$ It seems that we do not have direct, conscious access to motor programs, to syntax, to aspects of executive control (Crick 1995; Suhler \& Churchland 2009; TallonBaudry 2012; van Gaal et al. 2008), or to other kinds of "efference generators" (Grossberg 1999; Morsella \& Bargh 2010b; Rosenbaum 2002), including those for emotional systems (e.g., the amygdala; Anderson \& Phelps 2002; LeDoux 1996; Öhman et al. 2007; Olsson \& Phelps 2004). (Unconscious executive control from activated action sets exemplifies what has been historically referred to as "imageless," determining tendencies; Ach 1905/1951.)

In line with the sensorium hypothesis, examination of the liaison between action and consciousness reveals an isomorphism regarding what one is conscious of when one is (a) observing one's own action, (b) anticipating an action effect, (c) dreaming, and (d) observing the behaviors of others (Graziano 2010). In every case, it is the same, perceptual-like dimension of the experience that constitutes what is consciously available (Farrer et al. 2008; Melcher et al. 2013; Morsella \& Bargh 2010b; Rizzolatti et al. 2008; Sperry 1952). Speech processing provides a compelling example. Consider the argument by Levelt (1989) that, of all of the processes involved in language production, one is conscious of only a subset of the processes, whether when speaking aloud or subvocalizing. (Language reveals that mechanisms in action production can be complex but unconscious, as in the case of syntax.) It is for this reason that, when speaking, one often does not know exactly which words one will utter next until the words are uttered or subvocalized following lexical retrieval (Levelt 1989; Slevc \& Ferreira 2006). For instance, in the phonological loop, it is the phonological representation and not, say, the motor-related, "articulatory code" (Ford et al. 2005) that one is conscious of during spoken or subvocalized speech (Buchsbaum \& D'Esposito 2008; Fodor 1998; Rizzolatti et al. 2008). It is for this reason that Buchsbaum (2013) concluded that, in the phonological loop, the "inner voice" (i.e., the articulatory code) cannot hear itself. Although there has been substantial debate regarding the nature of conscious representations (e.g., whether they are "analogical" or "propositional"; Markman 1999), few would argue about the isomorphism among the conscious contents experienced while acting (e.g., saying "hello"), dreaming (e.g., saying "hello" in a dream), or observing the action of another (e.g., hearing "hello").

3.1.1. Perceptual-like contents as the lingua franca of action systems. Building on the sensorium hypothesis, we encountered a second reason why conscious contents must be perceptual-like. This reason pertains to the nature of the representational format. Regarding collective influence, the format of conscious contents must permit the contents to influence action systems (Freeman 2004) if there is to be perception-to-action translations (Merker 2012; W. Prinz 2003b). With this in mind, one would expect that the nature of representations involved in consciousness would be capable of being received and "understood" (i.e., to be access general; Barrett 2005) by multiple action systems in the brain. The perceptual-like representations discussed above in section 3.1 happen to meet this criterion. It has been proposed a priori, and for reasons having nothing to do with the current theorizing, that the representations that are the most "broadcastable" (i.e., received and understood by the most brain systems) happen to be perceptual in nature (Fodor 1983; Morsella \& Bargh 2010b; Morsella et al. 2009b). ${ }^{12}$ Moreover, one could argue that, if contents are aimed at influencing the complex and unconscious action mechanisms of the skeletomotor output system, it makes sense that the format of these contents would be the format to which the skeletomotor output system evolved to respond (i.e., perceptual stimuli). Accordingly, the phylogenetically old response systems in the skeletomotor output system (e.g., allowing for a spider stimulus to trigger a startle response; Rakison \& Derringer 2008) are likely to have evolved to deal with this kind of representation (i.e., one reflecting external objects; Bargh \& Morsella 2008; LeDoux 1996). Thus, perceptual-like representations can be construed as a kind of (domain general) lingua franca that can lead to content-driven activations in the skeletomotor output system. In other words, the mechanisms in the skeletomotor output system do not possess access specificity to contents in the conscious field (because they have access to all of the contents in the conscious field), but they do possess processing specificity (as each action mechanism can be activated by only some contents; Barrett 2005).

\subsection{Tenet: Conscious contents can directly activate action processes in the skeletal muscle output system}

According to ideomotor theory (Greenwald 1970; Harleß 1861; Hommel 2009; Hommel et al. 2001; James 1890; Lotze 1852), the perceptual representations identified by 
the sensorium hypothesis provide a mechanism for goaldirected action control. In this theory, the mental image of the (perceptual-like) action effects (in the body or in the world) of an instrumental action leads to the execution of that action, with the motor programming involved being unconscious. (It is noteworthy that contemporary ideomotor accounts are agnostic regarding the role of consciousness in action control [e.g., Hommel 2013].)

In ideomotor accounts, action selection is thus driven by the selection of the representation of the perceptual consequences of a motoric act. Hence, the many conscious contents about the world and the body can be construed as "action options" for the skeletomotor output system. From this standpoint, the urge to move the arm leftward is isomorphic to the perceptual consequences of what would be observed if the act were performed. This is also the case for the "higher" abilities, such as language. For example, before making an important toast (or making a toast in an unmastered language), a person has conscious imagery regarding the words to be uttered. Thus, action selection is concerned with achieving a final end state (e.g., flicking a switch or saying "hello"), which can be realized in multiple ways, as in the case of motor equivalence (Lashley 1942), in which several different behaviors can lead to the same end state. The unconscious motor programs realizing these end states are complex and context sensitive, as in the case of co-articulation in speech (Levelt 1989; see also Zhang \& Rosenbaum 2008).

According to ideomotor theory, there is a direct link between activation of action-related perceptual processes and (unconscious) action systems. Such a link is consistent with overwhelming evidence demonstrating that the presentation of action-related perceptual stimuli automatically and systematically influences action processing (see reviews of evidence in: Ellis 2009; Hommel \& Elsner 2009). This is evident in classic paradigms such as the flanker (Eriksen \& Eriksen 1974) and Stroop tasks (Stroop 1935). In the latter, participants must name the color in which words are written. When the color and word name do not match (e.g., RED in blue font), response interference arises because the automatic (and unintentional) word-reading plan competes with the weaker (and intended) colornaming plan (Cohen et al. 1990). Behavioral and psychophysiological evidence reveals that, during such response interference, competition involves simultaneous activation of the brain processes associated with both the targetand distracter-related responses (Coles et al. 1985; DeSoto et al. 2001; Eriksen \& Schultz 1979; Mattler 2005; McClelland 1979; van Veen et al. 2001). Additional evidence stems from neurological conditions (see review in Morsella \& Bargh 2011) and in the aforementioned research on unconscious efference binding, in which subliminal stimuli influence motor responses (Hallett 2007).

\subsection{Tenet: Action selection as the result of inter- representational dynamics}

From an ideomotor standpoint, once an action goal (e.g., pressing a button) is selected, unconscious motor efference enacts the action directly. ${ }^{13}$ From this standpoint, that which prevents the activation of an action goal representation from directly influencing overt action is only the activation of an incompatible action goal (James 1890; W. Prinz et al. 2009). In this framework, conscious representations of one's finger flexing, for instance, automatically lead to the flexing of one's finger, unless representations of incompatible action effects (e.g., the finger not flexing; James 1890) happen to be activated. It is important to note that the incompatibility regarding these two action effects resides, not in the conscious field, in which both action effects could be represented simultaneously, but rather in the simultaneous execution of the two action plans.

Consistent with this view of action conflicts, in one scenario, a conflict may involve representations $A$ and $B$ (associated with neural correlates $A_{\mathrm{NC}}$ and $B_{\mathrm{NC}}$ ), and then, at a later time and in a different context, a conflict may involve representations $C$ and $D$ (associated with neural correlates $C_{\mathrm{NC}}$ and $D_{\mathrm{NC}}$; Curtis \& D'Esposito 2009). Importantly, the two conflicts involve separate cognitive and neural processes, suggesting that "no single area of the brain is specialized for inhibiting all unwanted actions" (Curtis \& D'Esposito 2009, p. 72). Instead, representations, including those of action sets (Fuster 2003; Grafman \& Krueger 2009) and rules (E. K. Miller 2000) compete for the control of action. Such competition between actionrelated representations is evident in the aforementioned Stroop Task (Stroop 1935).

In our approach, this arrangement in which the contents of the conscious field lead to the activation of multiple (and often competing) action plans causes one to appreciate that, in the skeletomotor output system, there must be a (unconscious) mechanism by which one action plan can influence behavior more than other activated action plans. Such a mechanism would ensure that when holding one's breath while underwater, for example, the action plan to refrain from inhaling would influence behavior more than that of inhaling, although the conscious field would represent both inclinations. Appreciation of such potential "bottlenecks" in action selection can serve as a valuable constraint on theorizing regarding the neural structures underlying consciousness.

Importantly, in the perception-to-action loop, consciousness represents conflicts and not necessarily the representations associated with the resolution of such conflicts, should such representations exist (Morsella 2005). This peculiar property of consciousness arises because consciousness is about a stage of processing reflecting action options and not the mechanisms that, should they exist, represent conflict resolution. This illuminates why Chomsky (1988) observes that humans, unlike machines, are not only compelled to act one way or another but also can be inclined to act a certain way. Again, such inclinations could be construed as action options. The resolution of conflict depends not on some general property of consciousness, but on the peculiarities (e.g., relative strengths) of the systems that happen to be in conflict (Skinner 1953). Consciousness only permits that conflicts occur; it does not aim to resolve them (Morsella 2005). Each conflict is idiosyncratic and, if it is to be resolved, must require post-conscious, content-specific algorithms (e.g., one in which overt behavior is influenced most by prepotent action plans; Gold \& Shadlen 2007; Logan et al. 2015). Hence, it is challenging to arrive at general principles for predicting the outcomes of conflicts involving different systems (Campbell \& Misanin 1969; Krauzlis et al. 2014; see model of countermanding in Logan et al. [2015]). The Internet provides a good analogy for the role of consciousness in conflict: The Internet permits two people 
Morsella et al.: Homing in on consciousness in the nervous system

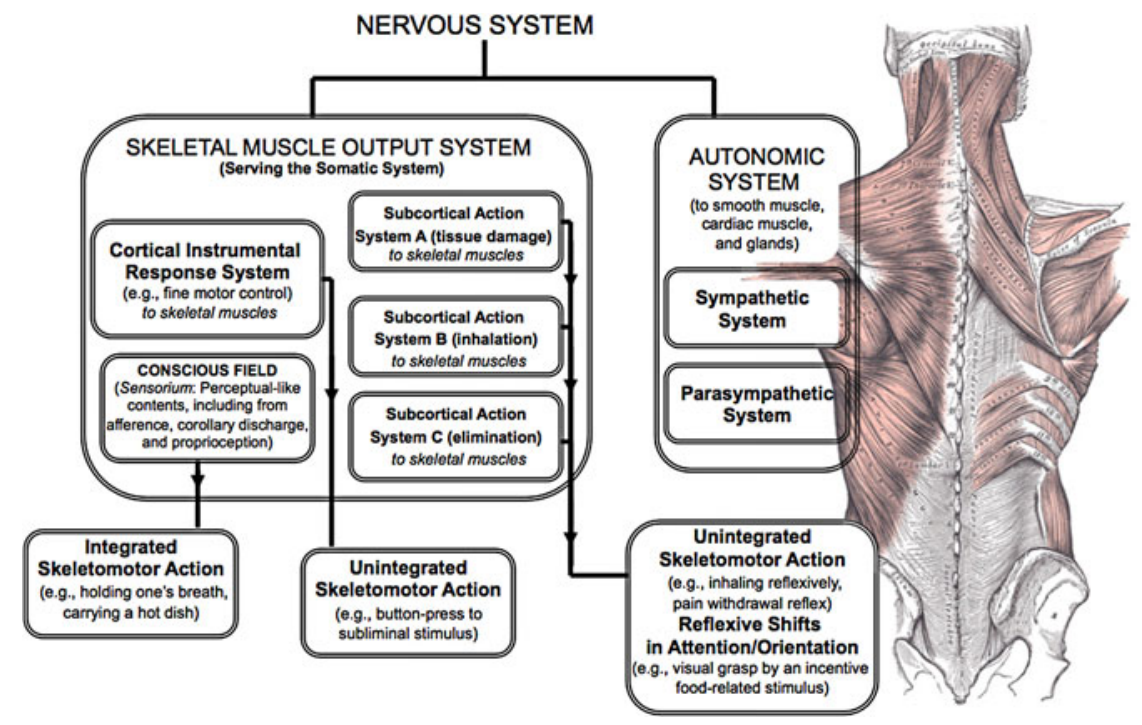

Figure 1. The divisions of the nervous system and place of consciousness within the system (based on Poehlman et al. 2012), including the major divisions of the Somatic and Autonomic systems. Afference binding within systems can be unconscious. Although response systems can influence action directly, as in the case of unintegrated actions, only in virtue of consciousness can multiple response systems influence action collectively, as when one holds one's breath while underwater.

from different cities to debate, but it cannot resolve conflicts between them. Another analogy would be an interpreter that translates for two parties which are in conflict about some issue. The interpreter is necessary for the instantiation of the conflict and for its potential resolution; the interpreter, however, cannot resolve the conflict.

In summary, to advance the identification of the neural substrates of consciousness, it is essential to keep in mind that consciousness is a phenomenon associated with perceptual-like processing and interfacing with the somatic nervous system (Fig. 1).

\subsection{Neural evidence supports the sensorium hypothesis}

The sensorium hypothesis and ideomotor theory reveal that, in terms of stages of processing, that which characterizes conscious content is the notion of perceptual afference (information arising from the world that affects sensoryperceptual systems; Sherrington 1906) and corollary discharges (e.g., when subvocalizing; cf. Chambon et al. 2013; Christensen et al. 2007; Jordan 2009; Obhi et al. 2009; Scott 2013), both of which are cases of perceptuallike content. This hypothesizing is consistent with the idea that, insofar as consciousness must always contain some content (Brentano 1874; Fodor 1980; 1998; J. A. Gray 1995; 2004; Hume 1739/1888; Koch 2004; Schopenhauer 1818/1819; Sergent \& Dehaene 2004), then it is parsimonious to propose that the neural regions responsible for processing that content must be part of the neural correlate of consciousness for that content. Thus, if content $X$ is in consciousness, then the circuits processing content $X$ must be part of a neural correlate of consciousness (e.g., at least of $X$ ). (Of course, within such an arrangement, it may be that the region[s] processing the particular content need not be the region $[\mathrm{s}]$ in which that content becomes associated with the conscious field; content processing could arise in one locus of the network, but the participation of contents in the conscious field could arise at another locus of the network.) With this notion in mind, we turn to the neural evidence regarding conscious contents.

Consistent with the sensorium hypothesis, there is evidence implicating perceptual brain regions as the primary regions responsible for consciousness. For example, direct electrical stimulation of parietal areas gives rise to the conscious urge to perform an action, and increased activation makes subjects believe that they actually executed the corresponding action, even though no action was performed (Desmurget et al. 2009; Desmurget \& Sirigu 2010; see also Farrer et al. 2008). However, activating motor areas (e.g., premotor regions) leads to the expression of the actual action, but subjects believe that they did not perform any action whatsoever (see also Fried et al. 1991). Importantly, consistent with our foregoing conclusions, the urge to perform a motor act is associated with activation of perceptual regions.

In accord with the sensorium hypothesis, the majority of studies involving brain stimulation and consciousness have found that stimulation of perceptual (e.g., posterior) brain areas leads to changes in consciousness (e.g., haptic hallucinations). This should not be surprising given that these regions were identified as "perceptual" in the first place by the use of self-report during brain stimulation (e.g., Penfield \& Roberts 1959). Self-report usually involves consciousness (see discussion in Bayne 2013). In the literature, we found only one datum in which brain stimulation of a frontal (non-olfactory) area led to a conscious content. In this study (Fried et al. 1991, cited in Haggard 2008), weak electrical stimulation of the pre-supplementary motor area led to the experience of the urge to move a body part, with stronger stimulation leading to movement of the same body part. It has been proposed that such activation led to corollary discharge that was then "perceived" by perceptual areas (Chambon et al. 2013; Farrer et al. 2008; Iacoboni 2005; Iacoboni \& Dapretto 2006; Lau et al. 2007; Melcher et al. 2013; Scott 2013), which would be consistent with the sensorium hypothesis. One strong hypothesis from this line of theorizing is that 
Morsella et al.: Homing in on consciousness in the nervous system

activations in regions that are non-perceptual or motor should never (independent of corollary discharge) influence the conscious field.

Consistent with the sensorium hypothesis and ideomotor theory, research reveals that a key component of the control of intentional action is feedback about ongoing action plans to perceptual areas of the brain, such as post-central cortex (Berti \& Pia 2006; Chambon et al. 2013; Desmurget et al. 2009; Farrer et al. 2008; Iacoboni 2005; Miall 2003). With this information in mind, it has been proposed that consciousness is associated not with frontal or higher-order perceptual areas, but with lowerorder perceptual areas (J. R. Gray et al. 2013; Liu et al. 2012; Tallon-Baudry 2012). However, it is important to qualify that though the sensorium hypothesis specifies that consciousness involves neural circuits that, traditionally, have been associated with perception, such circuits are widespread throughout the brain and exist within both cortical and subcortical regions (Merker 2012). Hence, the sensorium hypothesis is consistent with several neuroanatomical accounts of consciousness, including cortical, subcortical (e.g., thalamic), and thalamocortical accounts of consciousness. Thus, on the basis of the sensorium hypothesis alone, it is premature to dismiss subcortical accounts of consciousness (e.g., Merker 2007; Penfield \& Jasper 1954; Ward 2011).

In conclusion, at the present stage of understanding, the literature provides no clear answer regarding the neural substrates of any kind of conscious content (see treatment in Merker 2013b; 2013c). Based on the foregoing conclusions about conscious contents, we believe that, to illuminate this issue further, progress can be made by adopting an EASE perspective and focusing on a (relatively) tractable perceptual region - namely, that of the understudied olfactory system.

\subsection{Tenet: The olfactory system provides clues regarding the neural correlates of conscious perceptual content in the sensorium}

Our EASE perspective led us to the sensorium hypothesis. Now, with the same perspective, we focus on one kind of content in the sensorium. As noted in section 1.4, when attempting to isolate the substrates of a conscious content, researchers have followed Crick and Koch's (2003) recommendation and focused on vision. It is clear that isolating the neuroanatomical substrate of a visual conscious content remains controversial. From an EASE perspective, and based on previous research (Merrick et al. 2014; Morsella \& Bargh 2007), we focus our attention instead on olfaction (see also Keller 2011), a phylogenetically old system whose circuitry appears to be more tractable and less widespread in the brain than that of vision or higherlevel processing such as music perception. As Shepherd (2007) concludes, "the basic architecture of the neural basis of consciousness in mammals, including primates, should be sought in the olfactory system, with adaptations for the other sensory pathways reflecting their relative importance in the different species" (p. 93).

Several features of this system render it a fruitful arena in which to isolate the substrates of consciousness. First, olfaction involves a primary processing area that consists of paleocortex (which contains only half of the number of layers of neocortex) and primarily only one brain region (the frontal cortex; Shepherd 2007). In contrast, vision and audition often involve large-scale interactions between frontal cortex and parietal cortices. These observations reveal the relative simplicity of the anatomy of the olfactory system compared to that of other systems. Second, regarding the cortical-subcortical controversy, olfaction can reveal much about the contribution of thalamic nuclei in the generation of consciousness: Unlike most sensory modalities, afferents from the olfactory sensory system bypass the first-order, relay thalamus and directly target the cortex ipsilaterally (Shepherd \& Greer 1998; Tham et al. 2009). This minimizes spread of circuitry, permitting one to draw conclusions about the necessity of first-order thalamic relays in (at least) this form of consciousness.

By studying olfaction, one can also draw some conclusions about second-order thalamic relays (e.g., the mediodorsal thalamic nucleus $[\mathrm{MDNT}])$. After cortical processing, the MDNT receives inputs from olfactory cortical regions (Haberly 1998). Although it is likely that the MDNT plays a significant role in olfactory discrimination (Eichenbaum et al. 1980; Slotnick \& Risser 1990; Tham et al. 2011), olfactory identification, and olfactory hedonics (Sela et al. 2009), as well as in more general cognitive processes including memory (Markowitsch 1982), learning (Mitchell et al. 2007), and attentional processes (Tham et al. 2009; 2011), we have found no evidence that a lack of olfactory consciousness results from lesions of any kind to the MDNT (see theorizing about this possibility in Plailly et al. [2008]). Regarding second-order thalamic relays such as the MDNT, one must keep in mind that, in terms of circuitry, these nuclei are similar in nature to first-order relays (Sherman \& Guillery 2006), which are quite simple compared to, say, a cortical column.

Consistent with "cortical" theories of consciousness, Cicerone and Tanenbaum (1997) observed complete anosmia (the loss of the sense of smell) in a patient with a lesion to the left orbital gyrus of the frontal lobe. In addition, a patient with a right orbitofrontal cortex (OFC) lesion experienced complete anosmia ( $\mathrm{Li}$ et al. 2010), suggesting that the OFC is necessary for olfactory consciousness. (It is worth mentioning that we are speaking of the OFC with respect to, not the high-level executive processes with which it has been associated, but, consistent with the sensorium hypothesis, its perceptual processing [i.e., olfactory perception].) Moreover, conscious aspects of odor discrimination have been attributed to the activities of the frontal and orbitofrontal cortices (Buck 2000). Keller (2011) concludes, "There are reasons to assume that the phenomenal neural correlate of olfactory consciousness is found in the neocortical orbitofrontal cortex" (p. 6; see also Mizobuchi et al. 1999). (According to Barr and Kiernan [1993], olfactory consciousness depends on the piriform cortex.) However, not all lesions of the OFC have resulted in anosmia: Zatorre and Jones-Gotman (1991) reported a study in which OFC lesions yielded severe deficits, yet all patients demonstrated normal olfactory detection.

Another output pathway from the piriform cortex projects to the insular cortex (Haberly 1998; Schoenbaum \& Eichenbaum 1995), a structure that has anatomical connections to the ventral posteromedial (VPM) nucleus of the thalamus (Price et al. 1991). In light of (a) this information, (b) the conclusions presented above about the MDNT, and (c) theories in which thalamic structures play an important role in consciousness (e.g., Joliot et al. 1994; Llinás \& 
Morsella et al.: Homing in on consciousness in the nervous system

Ribary 2001; Llinás et al. 1998; Ward 2011), one could propose that olfactory consciousness depends on the integrity of the insula and thalamus. However, regarding the thalamus, it has been observed that, though thalamic lesions can impair olfactory discrimination and complex olfactory learning (Eichenbaum et al. 1980; Martin 2013), such lesions, including those of the VPM, never result in anosmia (Martin 2013; Price 1985; Price et al. 1991; Sela et al. 2009). The lesion literature also reveals an additional important fact about olfactory consciousness. Olfactory consciousness does not require the involvement of any transthalamic pathway. In addition, for corticocortical connections, the olfactory system requires no "higher-order" (Sherman \& Guillery 2006) thalamic relays (e.g., the MDNT or VPM; Gottfried 2006; Price 1985; Price et al. 1991). Considering these characteristics, Gottfried (2006) concludes, "The most parsimonious explanation for this anatomical variation is an evolutionary one: As primitive paleocortex, the olfactory circuitry simply developed long before the emergence of a thalamic module" (p. 53). These peculiar neuroanatomical characteristics are unique to olfactory consciousness.

Regarding the role of the insula in olfactory consciousness, after reviewing the literature, we concur with Mak et al. (2005) that there is no evidence that anosmia results from damage of any kind (e.g., unilateral or bilateral lesions) to the insular cortex: "There are no reports of olfactory deficits resulting from damage to the insula" (p. 1693; see also Damasio et al. 2012; Philippi et al. 2012; Tranel \& Welsh-Bohmer 2012).

Taken together, the neuroanatomical evidence presented above leads one to conclude that, in order to advance the current understanding of the neural underpinnings of consciousness, the next hypothesis to falsify is that olfactory consciousness requires cortical processes. This hypothesis is far from obvious, and it is falsifiable, because there are strong empirically based frameworks (e.g., Damasio 1999; Merker 2007; Panksepp 1998) proposing that consciousness is a function of subcortical processes. When these frameworks are integrated with our present treatment of the liaison between consciousness and olfactory circuits, our hypothesis could be proven to be inaccurate. For example, it might be that olfactory percepts are elaborated at a cortical level but become conscious only at some subcortical level (e.g., in the brainstem). Such a falsification of our hypothesis would advance our understanding of consciousness and the brain. Figuratively speaking, falsifying this particular "cortical" hypothesis provides the "lowest hanging fruit" for identifying the neural substrates of consciousness. In this way, the olfactory system can be used as a test-bed for hypotheses stemming from the cortical-subcortical controversy.

Third, from an EASE perspective, there are phenomenological and cognitive/mechanistic properties that render this system a fruitful network in which to investigate consciousness. Regarding phenomenological properties, unlike what occurs with other modalities, olfaction regularly yields no subjective experience of any kind when the system is under-stimulated, as when odorants are in low concentration, or during sensory habituation. This "experiential nothingness" (Morsella et al. 2010) is more akin to the phenomenology of the blind spot than to what one experiences when visual stimulation is absent (darkness). In the latter case, there still exists a conscious, visual experience (e.g., that of a black field). The experiential nothingness associated with olfaction yields no conscious contents of any kind to such an extent that, absent memory, one in such a circumstance would not know that one possessed an olfactory system. Hence, for our purposes, the creation of a conscious olfactory content is a true "addition" to the conscious field in that, not only does it involve the introduction of information about a particular stimulus, but also it involves the addition, from one moment to the next, of an entire modality. (See additional advantages of studying olfactory consciousness in Note 1.)

For these reasons, olfaction provides the best portal for understanding the neural correlates of additions to the conscious field. In our "creature in the cave" example, the smell of smoke is an addition to the conscious field that influences skeletomotor responses toward other conscious contents (e.g., the visual percept of the opening). Examining the neural correlates of such an addition might provide more evidence for the integration consensus. For example, it has been hypothesized that one becomes conscious of an olfactory percept only when the representation is part of a wider network involving other systems (Cooney \& Gazzaniga 2003), such as motor (Mainland \& Sobel 2006) or semantic-linguistic (Herz 2003) systems. (See review of the relationship between neural oscillations and olfactory consciousness in Merrick et al. [2014].)

In conclusion, regarding neuroanatomy, our primary hypothesis is that consciousness is associated with what has traditionally been regarded as "perceptual" regions of the brain, a hypothesis that challenges some accounts of consciousness in which consciousness is associated with executive processes in frontal cortex (e.g., Boly et al. 2011; Dehaene \& Naccache 2001; Lau 2008; Panagiotaropoulos et al. 2012; Safavi et al. 2014; Velly et al. 2007). Our secondary hypothesis is that olfactory consciousness can be constituted entirely by cortical circuits.

\section{The generation of conscious contents and field dynamics}

\subsection{Tenet: Content generation is encapsulated}

In our "creature in the cave" example, the addition of an olfactory content to the conscious field just "happens," without any noteworthy effort on the part of the organism (Mainland \& Sobel 2006). The content arises from a particular configuration of afference (e.g., the unconscious visual and auditory afference in the McGurk effect) to what can be construed as a content generator (associated with a perceptual region). Traditionally, these content generators (e.g., for color) have been construed as "modules" (Fodor 1983). Such a configuration of afference may include not only bottom-up afference, but also afference from unconscious top-down processes from knowledge systems and from frontal control regions (Suhler \& Churchland 2009; Tallon-Baudry 2012). Importantly, these generative processes that create conscious content are themselves context sensitive and unconscious (e.g., as in the McGurk effect; Lamme \& Spekreijse 2000). Regarding context sensitivity, consider that the image of a snake on a television screen triggers little if any fear, but such is not the case in a natural context.

Usually, contents enter consciousness in a manner that is more automatic, and less driven by intentions of the experiencing "agent," than appears to be the case in the everyday life of us pensive humans (Tallon-Baudry 2012; 
Morsella et al.: Homing in on consciousness in the nervous system

Vierkant 2013). Often, contents tend to "just happen" (Vierkant 2013). In line with these views, Helmholtz (1856/1961) proposed that unconscious processes can generate conscious content in a manner that resembles reflexes and other unintentional actions. When speaking about such "unconscious inferences," Helmholtz was referring not only to the generation of the conscious contents associated with low-level perceptual processes such as depth perception, but also to higher-level, non-perceptual processes such as automatic word reading - an unnatural, intellectual process that requires years of training. Helmholtz noted that when one is confronted with an orthographic stimulus (e.g., HOUSE), the stimulus automatically triggers a conscious representation of the phonological form of the word (i.e., /haus/). Seldom in everyday life is it appreciated that, in this situation, the visual stimulus triggers a conscious content that is very different in nature from that of the environmental stimulation that brought the content into existence: The conscious representation of the phonological form of the word is associated not with the visual modality, but with audition (Levelt 1989).

Conscious content can be generated by unconscious inferences also in the case of action-related urges (e.g., from unconsciously generated corollary discharge). These urges are often triggered in a predictable and insuppressible manner. For example, when one holds one's breath while underwater or runs barefoot across the hot desert sand in order to reach water, one cannot help but consciously experience the inclinations to inhale or to avoid touching the hot sand, respectively (Morsella 2005). Regardless of the adaptiveness of the expressed actions, the conscious strife triggered by the external stimuli cannot be turned off voluntarily (Morsella 2005; Öhman \& Mineka 2001). In these cases, the externally activated action-related urges are, in a sense, insulated, or "encapsulated" (Fodor 1983), from voluntary control. Thus, although inclinations triggered by external stimuli can be behaviorally suppressed, they often cannot be mentally suppressed (Bargh \& Morsella 2008). One can think of many cases in which externally triggered conscious contents are more difficult to control than overt behavior (Allen et al. 2013).

It has been argued that it is adaptive for content generation to be encapsulated in this way and for conscious contents to be incapable of directly influencing each other in the conscious field (Firestone \& Scholl 2014; Merrick et al. 2014; Rolls et al. 1977). From this standpoint, the conscious, perceptual representations for instrumental action should be unaffected by the organism's beliefs or motivational states (Bindra 1974; 1978). As Rolls and Treves (1998) conclude, "It would not be adaptive, for example, to become blind to the sight of food after we have eaten it to satiety" (p. 144). Similarly, it would not be adaptive for contents pertaining to incentive/motivational states to be influenced directly by other contents, such as desires and beliefs (Baumeister et al. 2007). For example, if one's beliefs could lead one to voluntarily "turn off" pain, guilt, or hunger, then these negative states would lose their adaptive value. Although motivation and beliefs may contaminate higher-order processes such as memory, they should have little influence over perceptual contents (Cooper et al. 2012; Firestone \& Scholl 2014; Pylyshyn 1984; 1999). Such "cross-contamination" across contents would compromise the critical influence of such incentive/motivational states on behavior.
Thus, each content is independent of other contents in the conscious field, whether the contents arise from environmental stimulation or from memory. Specifically, a conscious content (e.g., "da" in the McGurk effect) cannot directly influence the nature of other contents already in the conscious field (e.g., the smell of a rose, a toothache; Morsella 2005). (Of course, this is not to mean that the configuration of afference engendering one content cannot influence the generation of other contents - a form of context sensitivity in afference processing that occurs unconsciously [Lamme \& Spekreijse 2000; Merker 2012].) Because of encapsulation, illusions persist despite one's knowledge regarding the actual nature of the stimuli (Firestone \& Scholl 2014; Pylyshyn 1984).

It could be said that a given content does not "know" about its relevance to other contents (including highlevel, knowledge-based contents) or to current action. When representing a food object, for example, the content does not know whether the food item will be eaten or, instead, be thrown as a weapon. This view stands in contrast to several influential theoretical frameworks in which both the activation of, and nature of, conscious contents are influenced by what can be regarded as overarching goals or current task demands (e.g., Banerjee et al. 2012; Bhalla \& Proffitt 1999; Bruner 1973; Bruner \& Postman 1949; Dehaene 2014; Meier et al. 2007; Stefanucci \& Geuss 2009). Because of the principle of encapsulation, conscious contents cannot influence each other either at the same time nor across time, which counters the everyday notion that one conscious thought can lead to another conscious thought.

In the present framework, not only do contents not influence each other in the conscious field, but also as Merker (personal communication, June 30, 2012) concludes, content generators cannot communicate the content they generate to another content generator. For example, the generator charged with generating the color orange cannot communicate "orange" to any other content generator, because only this generator (a perceptual module) can, in a sense, understand and instantiate "orange." Hence, if the module charged with a particular content is compromised, then that content is gone from the conscious field, and no other module can "step in" to supplant that content (Kosslyn et al. 2001). As Merker notes, in constructing the conscious field, modules can send, not messages with content, but only "activation" to each other (see also Lamme \& Spekreijse 2000). This activation, in turn, influences whether the receiver module will generate, not the kind of content generated by the module from which it received activation, but rather its own kind of content (e.g., a sound). Because messages of content cannot be transmitted to other content generators, the neural correlates of the content for $X$ must include activation of the module that generates $X$, because a given content cannot be segregated from the process by which it was engendered, as stated previously.

\subsection{Tenet: Field contents must meet multiple-constraint satisfaction, be unambiguous, and appear as if apprehended from a first-person perspective}

From an EASE perspective, one can ask: What does a conscious content require if it is to lead to adaptive action? To answer this question, one must first consider that, in the 
case of object perception (such as the opening in our "creature in the cave" example), representations must be veridical to some extent in order to render action adaptive (Firestone \& Scholl 2014). For example, during action selection, it would not be adaptive for a circular object to be represented with, say, straight lines and corners. Similarly, it would not be adaptive for an object on the left to be represented as if it were on the right. Therefore, for the conscious field to afford adaptive action, it must represent with some veracity the spatial relation of those objects to the organism (Gibson 1979). Under normal circumstances, the contents of the conscious field at each moment are complete and unambiguous. Accordingly, Merker (2012) concludes that, because of the very nature of the constitution of the systems giving rise to conscious sensory representations, these systems are incapable of representing stimulus ambiguity (e.g., as in the Necker cube), at least at one moment in time. (However, such ambiguity could exist in unconscious perceptual processing [Merker 2012].) Thus, a given content emerges from polysensory configurations of afference, as in the McGurk effect, leading to the "global best estimate" of what that content should be (Helmholtz 1856/1961; Merker 2012).

Such well-constructed contents could stem from (a) the proposed, unconscious mechanisms of "multiple drafts" (Dennett 1991); (b) the interpretative processes of "apperception" (Wundt 1902/1904); or (c) "reentrant processing," in which a module, in order to give rise to a conscious representation, must receive feedback activation from other modules about that representation (Lamme 2001; Pascual-Leone \& Walsh 2001; Tong 2003). For example, if visual modules $X$ and $Y$ construct a representation which leads to the activation of other modules, then that representation becomes conscious only after feedback activation from the other modules returns to $X$ and $Y$ (Di Lollo et al. 2000; Fahrenfort et al. 2007; Goodhew et al. 2012; Grossberg 1999; Hamker 2003; Kriegel 2007; Lamme 2001; Lee et al. 2009; Llinás et al. 1998; Pascual-Leone \& Walsh 2001; Tong 2003). Re-entrant processing may instantiate a kind of "checks-and-balances" system for constructing accurate conscious contents that satisfy the criteria of multiple modules, a form of multiple-constraint satisfaction (Dennett 1991; Merker 2012). In addition, feedback of this sort may underlie the phenomenon of "contextual modulation" (e.g., in figure-ground effects; Lamme \& Spekreijse 2000). More simply, this feedback may be necessary because conscious contents may require (a) high levels of activation (Kinsbourne 1996) or (b) sustained activation for a prolonged period (Lau 2009), both of which can be furnished by sustained reverberation (Hebb 1949). In summary, for a content generator to contribute to the conscious field and for its contents to be well-crafted, it may require the concurrent activation from both feed-forward and feedback mechanisms (Lamme \& Spekreijse 2000).

The conscious field of our "creature in the cave" includes representations of urges and external objects, which incorporate the relation between such things and the organism itself (Lehar 2003; Merker 2012; Yates 1985). More generally, contents are usually sensed to be different from, and separate from, the observing agent (Brentano 1874; Merker 2012; Schopenhauer 1818/1819). Insofar as the action selection process of the skeletomotor output system must take into account spatial distance from the organism as one of the many factors in adaptive selection, then all contents about the external world (including the body) must have a common, egocentric reference (Merker 2013c). It would be disadvantageous for this rule to be violated and for, again, an object on the left to be represented as if on the right. Hence, most conscious contents appear as if from a first-person perspective (Gibson 1979; Merker 2013c; J. Prinz 2007). The conscious field is imbued with this first-person perspective during waking, in dreaming, and for illusions in which, through clever experimental manipulations and the presentation of certain stimuli, the perspective is momentarily perceived as if from outside of the body (Ehrsson 2007). ${ }^{14}$ From this standpoint, the demands of adaptive action selection require the creation of a first-person perspective, which is a primitive form of "self."

\subsection{Tenet: The conscious field serves as a frame that represents encapsulated contents for collective influence over, not itself, but the skeletal muscle output system}

It seems that the conscious field is like a mosaic of discrete, heterogeneous contents, rendering the field to be combinatorial. Each content is well-crafted and unambiguous (Dietrich \& Markman 2003; Freeman 2004; Köhler 1947; Merker 2012; Scholl 2001). The contents cannot directly influence each other, ${ }^{15}$ and the content generators cannot duplicate the generative abilities of each other. Thus, the resultant contents from these modules are encapsulated from each other. These mosaic-like Gestalts (i.e., the conscious field) arise in consciousness in a discontinuous manner, with each conscious moment, lasting for fractions of a second, having an updated version of all of the contents in the conscious field. For action to be adaptive, the refresh rate of the entire field must be faster than the quickest rate at which voluntary actions can be produced. Hence, the refresh must occur more rapidly than the rate at which the fastest actions (e.g., saccades) can be emitted (Merker 2013c). (For theorizing regarding the temporal properties of such an updating process, see Libet [2004] and Merker [2012, p. 56; 2013c, p. 12].)

Importantly, the collective influence of the combination of contents in the conscious field is not toward the conscious field itself; instead, according to PRISM, the conscious field is apprehended by the (unconscious) mechanisms composing the skeletomotor output system. Thus, the conscious contents of blue, red, a smell, or the urge to blink are the tokens of a mysterious language understood, not by consciousness itself (nor by the physical world), but by the unconscious action mechanisms of the skeletomotor output system. Why do things appear the way they do in the field? Because, in order to benefit action selection, they must differentiate themselves from all other tokens in the field-across various modalities/ systems but within the same decision space.

Although possessing elements of a "Cartesian Theater" (Dennett 1991), this arrangement does not introduce the "homunculus fallacy," because, in the skeletomotor output system, there are many motor-homunculi, each incapable of duplicating the functions of the system as a whole (Dennett 1991). Unlike the "workspace" models associated with the integration consensus (e.g., Baars 1988; Dehaene 2014), which propose that conscious representations are 
Morsella et al.: Homing in on consciousness in the nervous system

"broadcast" to modules engaged in both stimulus interpretation and content generation, in our framework (as in Merker 2007), the contents of the conscious field are directed only at response modules in the skeletomotor output system. In short, conscious contents are "sampled" only by unconscious action systems that are charged with (specifically) skeletal muscle control.

\section{Passive frame theory: An action-based synthesis}

No activity of mind is ever conscious. - Lashley (1956, p. 4)

It is the result of thinking, not the process of thinking, that appears spontaneously in consciousness.

$$
\text { — George A. Miller (1962, p. 56) }
$$

To reiterate, progress on the study of consciousness and the brain has suffered not so much from a lack of data, but from a lack of a suitable, internally coherent framework with which to interpret extant data (Grossberg 1987). To provide such a framework, we now synthesize all of our tenets.

Consciousness is a phenomenon serving the somatic nervous system (subset consensus); it is in the service of adaptive skeletomotor function (PRISM; see Figure 1). At each moment, the conscious field is generated anew, with a new medley of contents. PRISM predicts the kinds of information that must become conscious contents. These kinds of information are about things (e.g., external objects and urges) that should influence the skeletomotor output system (the "steering wheel" associated with consciousness). To the organism, these unambiguous, wellcrafted, and highly context-sensitive contents usually arise in an automatic, non-self-generated manner. The contents, which arise from configurations of afference (including topdown processes and unconscious intersensory interactions), are encapsulated from each other: One content does not "know" whether it is relevant to other contents or to ongoing action (encapsulation). Consciousness can thus be construed as a "continuous feed" system that is always "on," even in the absence of conflict or of other forms of cross-system checking (Morsella 2005). In other words, the primary function of the conscious field is collective influence of otherwise encapsulated contents on the skeletomotor output system. Such an influence is essential especially under conditions of conflict; however, as a continuous feed system, this mechanism of collective influence persists even under conditions in which conflict is absent.

The contents (e.g., objects and urges) are "perceptual-like," which is the "common format" apprehended by the actionrelated mechanisms composing the skeletomotor output system (sensorium hypothesis). Conscious contents are sampled only by unconscious action systems (PRISM). These contents can be construed as "action options." Absent conflict, these action options activate unconscious efferences to the skeletomotor output system (ideomotor theory). Unconscious mechanisms such as unconscious inferences and corollary discharges from activated action plans (e.g., in the phonological loop; Scott 2013) can trigger conscious contents. We refer to the interdependence between unconscious and conscious mechanisms as the conscious-unconscious cycle.

The conscious field permits collective influence of all of the heterogeneous contents upon the skeletomotor output system (PRISM). All influences on skeletomotor behavior, from the highest-level processes (e.g., language) to the lowest-level processes (e.g., pain withdrawal), must engage the skeletomotor output system.

For the selection of any skeletomotor plan to be adaptive, selection must transpire in the frame of the other conscious contents composing the conscious field at that instant. We refer to this as a "frame check." It is required for adaptive skeletomotor function, and is essential for integrated actions in the context of conflict. Under certain circumstances (e.g., fast motor acts such as rapid eye gazes), frame checks must occur quickly, as last-minute changes to courses of action often arise in the face of new information (Merker 2013c). Hence, frame checks must occur more rapidly than the rate at which the fastest actions (e.g., saccades) can be emitted (Merker 2013c). During adaptive action selection, anticipated action effects, actual action effects, and information about the immediate environment must exist as comparable tokens in a common decision-space. Although consciousness has historically been associated with the highest levels of processing, here it is revealed that consciousness must occur at the level of processing that is shared with that of representations of the immediate external environment. Consciousness is associated only with frame checks and not with the more active aspects of the conscious-unconscious cycle (e.g., content generation, conflict resolution, motor programming).

With these conclusions in mind, we now return to our "creature in the cave" scenario. Because of encapsulation, the percept of the opening of the cave is consciously available even when the opening is not relevant to ongoing action (i.e., before detection of the smoke). Regarding neural events, the content addition of the olfactory stimulus involves a wide network of regions (integration consensus). To the organism, the olfactory content "just happens." Before the content, there was no olfactory consciousness; hence, the smell of smoke is a "true addition." Because messages of content cannot be transmitted, the olfactory content must involve perceptual areas of processing (sensorium hypothesis). The neuroanatomical correlates of such an olfactory content remain controversial (e.g., the corticalsubcortical controversy). We believe that progress regarding such controversies will stem from further examination of the cortical aspects of olfactory consciousness.

For our "creature in the cave," the conscious content about the smell triggers a conscious content representing an avoidance tendency toward the smell. Specifically, this content about potential action is about the perceptual aspects of the to-be-produced action (sensorium hypothesis). Such a conscious content about an action can arise from activations in perceptual areas triggered by corollary discharges from unconscious, motor processes (Buchsbaum 2013; Chambon et al. 2013; Iacoboni 2005; Iacoboni \& Dapretto 2006; Lau et al. 2007). Again, as with the case of (a) anticipated action effects, (b) actual action effects, and (c) information about the immediate environment, adaptive action selection requires that the conscious contents associated with both Stage 1 (e.g., the percept of the opening and the warmth) and Stage 2 (e.g., the smell and the inclination to stay in the cave) be, in terms of their functional consequences for action selection, the same kind of thing-comparable tokens existing in the same decision space. Thus, the conscious field permits for 
Morsella et al.: Homing in on consciousness in the nervous system

the contents about the smell and about the opening to influence action collectively.

During the frame check, the content about the potential action to exit conflicts with the content about the inclination to remain within the warm enclosure. In this case, the conflict between remaining in the enclosure and exiting is consciously experienced by the organism; however, this is only one component of all that is transpiring within the mental apparatus as a whole. Representations reflecting the outcome of the conflict (should they exist) reside outside of consciousness (which itself is primarily about action options). Such a resolution will reflect not any property of consciousness, but the peculiarities of the conflicting systems involved.

In terms of action selection, the conscious field could be construed as the evolutionary strategy for dealing with what the ethologists and behaviorists referred to as a complex of multiple discriminative stimuli (also known as a compound discriminative stimulus), in which the "stimulus control" from one discriminative stimulus depends systematically on the nature of the other discriminative stimuli composing the scene (Spear \& Campbell 1979). In collective influence, the response to one conscious content - and the "meaning" of that content for ongoing action selection - depends exclusively on the nature of itself and the other conscious contents at that moment in time. Thus, the conscious field permits for the response to one content to be made in light of the other contents (Tsushima et al. 2006), thereby yielding integrated behavior. In this process, the conscious field wholly and exclusively determines what in everyday life is called voluntary behavior.

We now apply these insights to a case involving a higherlevel system (language). At Thanksgiving dinner, our simple organism (now equipped with language) perceives a stimulus that triggers (unintentionally and automatically) in consciousness the action option of uttering a famous saying. This perceptual-like subvocalized phonological content, which "just happens" in consciousness, could stem from complex processes, involving, perhaps, corollary discharge from unconscious motor centers (Mathalon \& Ford 2008; Scott 2013). After a frame check, the organism does not utter the phrase, because, a moment after experiencing the subvocalization, the organism experienced another content (the smell of hot chocolate) that led to an action plan (drinking) that was incompatible with speaking. The foregoing example reveals how the reiterative cycle of conscious field construction, and the frame check that each construction affords, yields the collective influence that adaptive skeletomotor action demands.

\section{Implications and concluding remarks}

Passive frame theory reveals that the province of consciousness in nervous function is more low-level, circumscribed, counterintuitive, and passive than what theorists have proposed previously. Because conscious contents do not know their relevance to other contents nor to ongoing action, consciousness is less purposeful at one moment in time than what intuition suggests. It is not only less purposeful and "all-knowing" than expected, but also contributes only one function (albeit an essential function) to a wide range of processes, much as how the Internet plays the same critical role for a varied group of events (e.g., the sale of books or dissemination of news), and the human eye, though involved in various processes (e.g., hunting vs. locomoting), always performs the same function. Because consciousness contributes to a wide range of heterogeneous processes, it appears as being capable of doing more than it actually does.

Passive frame theory also reveals that the contribution of consciousness to nervous function is best understood from a passive, structural-based (instead of a processing-based) approach. Such a perspective is in contrast to contemporary approaches but is in accord with historical ways to describe how biological systems achieve their ends (Grafman \& Krueger 2009). Figuratively speaking, at one moment in time, there are few "moving parts" in the conscious field. (The field itself has no memory and performs no symbol manipulation; for these high-level mechanisms, it only presents, to action systems, the outputs of dedicated memory systems and of executive processes, respectively.)

Compared to the many functions attributed to consciousness, that which is proposed here is by far the most basic, low-level function. Because consciousness integrates processes from various systems, this role is more apparent when studying consciousness across modalities than when studying it within only one modality. Hence, the province of consciousness is best appreciated from a "systems-level" approach to the brain. The conscious-unconscious cycle of our approach also reveals the interdependence between (passive) conscious and (active) unconscious processes. (Few approaches examine the interactions between the two kinds of processes.) Last, our approach reveals that the demands of adaptive action (e.g., heterogeneous action systems must use the same effector) and the limitations of the cognitive apparatus (e.g., action selection often must occur quickly), illuminate many of the properties of consciousness, including that of a basic form of the sense of self.

With our framework as a foundation, future investigations could lead to a consensus regarding, for example, the neural circuitry underlying consciousness. Because the identification of the dynamic, neural processes associated with consciousness depend in part on identification of the neuroanatomical correlates of consciousness, a consensus should first be reached regarding the latter, more tractable problem. The most feasible way to reach such an identification is for investigators to cooperate across fields and attempt to isolate the correlates of consciousness at multiple levels of analysis, with increased research activity devoted to regions (a) predicted a priori, by theory, to be associated with consciousness and (b) identified as being the most experimentally tractable, as in the case of olfactory circuits. Because consciousness serves as a special interface between sensory and motor processing, theory-based predictions regarding the relationship between conscious contents and the skeletomotor output system (e.g., the link between olfactory percepts and integrated skeletomotor behavior) may advance attempts to identify the substrates of consciousness. We hope that our framework serves as a useful foundation for such collective endeavors.

Together with our EASE perspective, passive frame theory provides a fecund and internally coherent framework for the study of consciousness. Based on hypotheses from diverse areas of investigation, our synthesis reveals how consciousness serves an essential, integrative role for the somatic nervous system, a role that is intimately related to adaptive functioning of skeletal muscle (one of many effector systems in the body). When not subscribing 
to an EASE perspective, one could imagine ways in which the proposed contribution of consciousness to the somatic system could be realized without anything like "subjectivity." However, these musings would reflect our human powers of imagination more than what was selected in evolution for nervous function to yield adaptive behavior.

\section{ACKNOWLEDGMENTS}

This article is dedicated to John Bargh, who reminded everyone about the power and sophistication of the unconscious. This research was supported by David Matsumoto and the Center for Human Culture and Behavior at San Francisco State University. We gratefully acknowledge Christina Merrick's assistance with the review of the olfaction literature, and Ricardo Morsella's guidance regarding the philosophical literature. We are also grateful for the advice and suggestions of T. Andrew Poehlman and Lawrence Williams, and for the assistance and support from Robert M. Krauss, Bruce L. Miller, John Paul Russo, Mark Geisler, Avi Ben-Zeev, Björn Merker, Carlos Montemayor, Allison Allen, Bernhard Hommel, Gary Marcus, J. Scott Jordan, Wolfgang Prinz, Bruce Bridgeman, and Chelsea Hunt. This project would not have been possible without the efforts of the members of the Action and Consciousness Laboratory.

\section{NOTES}

1. First, unlike in vision, there are few executive functions (e.g., mental rotation, symbol manipulation) coupled with olfaction. Hence, in olfaction, one is less likely to conflate the substrates of consciousness with those of high-level executive functions (see Aru et al. 2012; Panagiotaropoulos et al. 2013). Similarly, in vision and audition, imagery can be used to preserve information in working memory through active rehearsal (Baddeley 2007), but olfactory images are difficult to couple with such operations (Betts 1909/1972; Brower 1947; Lawless 1997; Stevenson 2009). Second, olfactory experiences are less likely to occur in a self-generated, stochastic manner: Unlike with vision and audition, in which visually rich daydreaming or "earworms" occur spontaneously during an experiment and can contaminate psychophysical measures (respectively), little if any self-generated olfactory experiences could contaminate measures. Last, olfaction is more segregated from the semantic system than is the most studied sensory system vision. In the latter, there are deep, inextricable relationships among perception, conceptualization, and semantics (Barsalou 1999; Kosslyn et al. 2006). Thus, when isolating the neural substrates of olfactory consciousness, one is less likely to include higher-level processes (e.g., semantic processes) associated with more than just olfactory consciousness. (See additional advantages of studying olfactory consciousness in section 3.5.)

2. Consistent with this consensus, evidence reveals that consciousness of some kind persists with the nonparticipation (e.g., because of lesions) of several brain regions (Morsella et al. 2010): cerebellum (Schmahmann 1998), amygdala (Anderson \& Phelps 2002; LeDoux 1996), basal ganglia (Bellebaum et al. 2008; Ho et al. 1993), mammillary bodies (Duprez et al. 2005; Tanaka et al. 1997), insula (Damasio 2011, as cited in Voss 2011; see also Damasio 2010), and hippocampus (Crick \& Koch 1990; Milner 1966; Postle 2009). In addition, investigations of "split-brain" patients (O'Shea \& Corballis 2005; Wolford et al. 2004) suggest that consciousness survives following the nonparticipation of the non-dominant (usually right) cerebral cortex or of the commissures linking the two cortices. Controversy surrounds the hypothesis that cortical matter is necessary for consciousness (discussed in sect. 2.3 and sect. 3.5).

3. For present purposes, unconscious events are those processes that, though capable of systematically influencing behavior, cognition, motivation, and emotion, do not influence the organism's subjective experience in such a way that the organism can directly detect, understand, or self-report the occurrence or nature of these events (Morsella \& Bargh 2010a).
4. See review in Morsella and Bargh (2011). In brief, unconsciously mediated actions can be observed during unconscious states, including forms of coma/persistent vegetative states (Kern et al. 2001; Klein 1984; Laureys 2005; Pilon \& Sullivan 1996) and epileptic seizures, in which automatisms arise while the actor appears to be unconscious. These unconscious automatisms include motor acts (Kokkinos et al. 2012; Kutlu et al. 2005), written and spoken (nonsense) utterances (Blanken et al. 1990; Kececi et al. 2013), singing (Doherty et al. 2002; Enatsu et al. 2011), and rolling, pedaling, and jumping (Kaido et al. 2006). Similarly, in neurological conditions in which a general consciousness is spared but actions are decoupled from consciousness, as in alien hand syndrome (Bryon \& Jedynak 1972; Chan \& Ross 1997), anarchic hand syndrome (Marchetti \& Della Sala 1998), and utilization behavior syndrome (Lhermitte 1983), hands and arms carry out complex actions autonomously. These actions include complex goal-directed behaviors such as object manipulations (Yamadori 1997). Often, the behaviors are unintentional (Marchetti \& Della Sala 1998; Suzuki et al. 2012). (See other forms of unconscious action in Bindra [1974], Milner and Goodale [1995], Weiskrantz [1992, 1997], and Westwood [2009].) In addition, actions can arise from stimuli of which the actor is unaware, as in the case of subliminal stimuli that, though imperceptible, can influence action (sect. 2.4; Ansorge et al. 2007; Hallett 2007).

5. In binocular rivalry (Logothetis \& Schall 1989), an observer is presented with different visual stimuli to each eye (e.g., an image of a house in one eye and of a face in the other). Surprisingly, however, an observer experiences seeing only one object at time (a house and then a face), even though both images are always present.

6. Regarding neural oscillations, for example, controversy remains about the role that they play in the generation of consciousness. It has been proposed that cortical electroencephalography does not reflect conscious processing (Merker 2013b). In addition, there is controversy concerning the regions that are responsible for these oscillations. Consciousness-related oscillations have been proposed to reflect primarily (a) thalamic activity (Ward 2011), (b) thalamocortical activity (Joliot et al. 1994; Llinás \& Ribary 2001; Llinás et al. 1998), and (c) corticocortical activity (Panagiotaropoulos et al. 2012; Schubert et al. 2006). (Regarding the role of oscillations in consciousness, see Aru \& Bachmann 2009; Crick \& Koch 1990; Doesburg et al. 2005; 2009; Engel \& Singer 2001; Fries 2005; Hameroff 2010; Jung-Beeman et al. 2004; Meador et al. 2002; Panagiotaropoulos et al. 2012; Singer 2011; Uhlhass et al. 2009; Wessel et al. 2012.)

7. Action selection, as when one presses one button versus another button or moves leftward versus rightward, is distinct from motor control/motor programming (Proctor \& Vu 2010), processes which are largely unconscious (discussed in sect. 3.1).

8. Experiments have revealed that incompatible skeletomotor intentions (e.g., to point right and left, to inhale and not inhale) produce systematic intrusions into consciousness (J. R. Gray et al. 2013; Molapour et al. 2011; Morsella et al. 2009c), but no such changes accompany conflicts involving smooth muscle (Morsella et al. 2009a) or occur at perceptual stages of processing (e.g., intersensory processing; see quantitative review of evidence from multiple paradigms in Morsella et al. [2011]). Additional evidence stems from the observation that consciousness is required to counteract the interference effects of conflict (Desender et al. 2014).

9. Bleuler (1924) proposed that that which transforms unconscious processes to conscious processes is more than just integration the integration must involve the "ego complex." We propose that this complex is related to volition and the skeletomotor output system.

10. It is important to specify the notion of an addition of content to the conscious field. It has been proposed that consciousness cannot be "content free" but must always possess some content (Brentano 1874; Fodor 1980; 1998; J. A. Gray 1995; 2004; Hume 1739/1888; Koch 2004; Schopenhauer 1818/ 1819; Sergent \& Dehaene 2004), such as that of a perceptual object or an action-related urge. We adopt this assumption. However, it has also been stated that contents enter 
consciousness, as if consciousness were a bucket into which contents enter. Thus, there is a contradiction: According to one interpretation, there can be no bucket without contents, but, according to the other interpretation, consciousness (i.e., the bucket) could exist independent of contents. Perhaps it is more accurate and parsimonious to state that new contents do not enter consciousness but become conscious, thereby joining other conscious contents. Hence, when something becomes conscious (e.g., the smell of smoke), we regard it as an "addition" to the conscious field. If consciousness is capacity-limited, then at times an addition may also be construed as a replacement, because the new content removes other content (see evidence in Goodhew et al. 2012). It is controversial whether contents in the conscious field actually constitute the field or modulate it. (For a treatment concerning whether the field is componential or unitary, see Searle [2000].) Importantly, in the present model, whether the field is componential or unitary leads to the same functional consequences, because of the encapsulation of conscious contents (sect. 4.1).

11. According to a minority of theorists (see list in James 1890 , p. 772), one $i$ s conscious of the efference to the muscles (what Wundt called the feeling of innervation; see James 1890 , p. 771). In contrast, James (1890) staunchly proclaimed, "There is no introspective evidence of the feeling of innervation" ( $p$. 775). This efference was believed to be responsible for action control (see review in Sheerer 1984). (Wundt later abandoned the feeling-of-innervation hypothesis; see Klein 1970.)

12. This proposal is based in part on the requirements of "isotropic information," which are beyond the scope of the present discussion (see Fodor 1983). As noted by Fodor (2001), in order to solve the "input" (or "routing") problem, in which the appropriate representations must be made available to the appropriate information-processing modules, the representations must be perceptual in nature (Barrett 2005; Barsalou 1999).

13. Perhaps, in addition to action selection, a "go signal" is required for action initiation (Bullock \& Grossberg 1988). The need for such a mechanism is beyond the scope of the present framework.

14. From an EASE perspective, it is parsimonious to treat the sense of agency, too, as a conscious content that is experimentally manipulable. It is experienced when there is the perception of a lawful correspondence between action intentions and action outcomes (Wegner 2002) and depends in part on conceptual processing (Berti \& Pia 2006; David et al. 2008; Haggard 2005; 2008; Jeannerod 2009; Synofzik et al. 2008).

15. In forms of metacognition (e.g., indirect cognitive control; Morsella et al. 2009b), there are cases in which, through top-down control, conscious contents can lead to the strategic activation of other contents; however, this mechanism is beyond the abilities of our simple organism and, for the present purposes, unnecessary for appreciating the primary role of consciousness.

\section{Open Peer Commentary}

\section{Can skeletomotor action integration occur without consciousness? Evidence from unconscious action inhibition}

doi:10.1017/S0140525X15001995, el69

\section{Alisabeth Ayars \\ Department of Psychology, University of Arizona, Tucson, AZ 85712. alisabetha@email.arizona.edu}

Abstract: Morsella et al. claim that consciousness functions to integrate incompatible skeletal muscle intentions. Their primary cases of conscious skeletomotor integration involve action suppression, such as holding one's breath underwater, which requires suppressing the urge to inhale. This indicates that the authors seem to think action inhibition counts as the relevant kind of integration. I suggest that there is reason to think this kind of inhibition can occur unconsciously via unconscious inhibitory mechanisms. Unconscious inhibition therefore potentially undermines the authors' thesis. If cases of unconscious inhibition are not instances of integration, then the authors should clarify exactly what counts as integration.

In the target article, authors Morsella et al. claim that consciousness functions to integrate "incompatible skeletal muscle intentions for adaptive action” (sect. 2.4, para. 5). The authors' claim is one of necessity: "[T]he conscious field is necessary to integrate what appear to be multiple inclinations toward the skeletomotor output system" (sect. 2.4, para. 5; emphasis mine). In this commentary, I identify a worry - namely, that the authors have not adequately defended the strong necessity claim. Necessity claims are inherently vulnerable: a single counter-example renders the claim false. I suggest that there is both prima facie reason to think that skeletomotor integration can occur unconsciously, and some positive empirical evidence that it can.

The authors note that many theories of consciousness specify the purpose of consciousness to be quite high level-for example, the maintenance of the self, semantic processing, or theory mind. In contrast, the authors think consciousness serves a "counterintuitive, passive, and "low level" function (target article, introductory para. 1). A risk of identifying the function of consciousness to be low-level is that it will turn out that the low-level task can be subserved by non-conscious processes. The more basic the role granted to consciousness is, the more likely that scientific investigation will reveal that the function can be achieved in the absence of consciousness.

To support the claim that consciousness is necessary for skeletomotor integration, the authors must convincingly argue that there are no cases of unconscious skeletomotor integration. Contrasting cases of obviously conscious integration like holding one's breath underwater with cases of unconscious (non-skeletomotor) integration like the McGurk effect is too weak a method to support the necessity claim. How do we know that there are not cases of unconscious skeletomotor integration of which we are not aware, given that such integration would be unconscious?

The authors do not give a precise definition of "integration," but they do give useful examples. Holding one's breath underwater requires integration. In this case, a reflexive action (inhaling) is inhibited by one's goals (wanting to survive). Action inhibition appears therefore to count as integration. If there are cases of unconscious action inhibition, then this would presumably mean there are cases of unconscious integration.

There is prima facie reason to think the relevant integration/conflict can occur unconsciously. The authors claim that conscious conflict occurs whenever there are multiple incompatible inclinations on skeletomuscles. On a weak reading of "inclinations," in which an inclination is simply an activated motor plan, the phenomenon of "incompatible skeletal muscle intentions" (sect. 2.4, para. 5) is not particularly rare. Motor plans are automatically activated to a wide range of stimuli, a point the authors acknowledge: "[There is] overwhelming evidence demonstrating that the presentation of action-related perceptual stimuli automatically and systematically influences action processing" (sect. 3.2, para. 3). Simply viewing or categorizing an object primes motor responses associated with the object, like grasping actions (Tucker \& Ellis 2004). These motor responses are clearly often incompatible with current goals: grasping reflexively at a stranger's personal effects would be quite deleterious, for instance. If motor plans are indeed activated as ubiquitously as the research suggests, then it is likely that incompatible actions - or even, "unconscious urges" - are activated and suppressed beneath conscious awareness all of the time. Perhaps the authors would counter that these incompatible inclinations are all conscious. However, it does not seem as if we are constantly bombarded with conscious urges to grasp the objects we 
encounter. The authors might also contend that action inhibition is not integration. But the primary examples of conscious integration that the authors offer, such as holding one's breath underwater, are cases in which a reflexive action is inhibited.

Consistent with the claim that the brain is constantly engaged in the suppression of unwanted incompatible motor plans, consider the effects of releasing these inhibitions. Under the influence of alcohol or other inhibition-releasing drugs, people behave quite differently-they are more aggressive, for example, and find themselves making inappropriate or irreverent comments. Although it often seems like the alcohol "created" or "implanted" these inclinations, it is plausible that they are present even while sober; however, because unconscious inhibitory mechanisms are in place to suppress them, they are not consciously experienced. Consider that we often find ourselves acting appropriately and in-context without any effort at all, such as when we are able to remain quiet at a movie or show. At first, one might accidentally talk at inappropriate times, but eventually, remaining quiet becomes less effortful or entirely un-effortful. This does not demonstrate that it is definitely the result of unconscious inhibition. But how do we know that it is not? The authors claim that there are no cases of this unconscious integration. If there is reason to think there might be unconscious integration, then the authors must explain why this is mistaken.

In addition to the concerns I have raised, there is positive empirical evidence that actions can be suppressed unconsciously. One widely discussed finding that is taken by many to evince unconscious inhibition is the negative compatibility effect. The negative compatibility effect refers to the ability of subliminal action-suggesting primes (such as a leftward arrow priming a left response) to render performance worse when a congruent or compatible response must be made to a target, under certain conditions. For instance, if a prime such as ">>" is subliminally presented prior to a target (">>") and there is a significant delay between prime and target, responses to congruent targets is slower than incongruent targets (Eimer \& Schlaghecken 2003; Schlaghecken et al. 2006). (The effect reverses with a shorter prime-target delay.) A standard explanation for the effect is that it involves an unconscious inhibitory mechanism that suppresses the activated motor response from the prime, in order to preempt interference from the prime on the response to the target (Jaskowski \& Przekoracka-Krawczyk 2005; Schlaghecken \& Eimer 2006; Sumner et al. 2007). This occurs entirely beneath subjects' awareness because the subjects are never aware of the prime. The negative compatibility effect therefore provides some positive evidence that consciousness is not required for skeletomotor integration.

\section{Content encapsulation in consciousness is likely to be incomplete}

doi:10.1017/S0140525X15002009, e170

\author{
Demis Basso ${ }^{\mathrm{a}, \mathrm{b}}$ \\ ${ }^{a}$ Faculty of Education, Free University of Bozen, 39042 Bressanone/Brixen \\ (BZ), Italy; ' ${ }^{b}$ Center for Applied Cognitive Neurosciences, 00174 Rome, Italy. \\ demis.basso@unibz.it \\ https://www.unibz.it/en/education/people/StaffDetails.html? \\ personid $=30397 \&$ hstf $=30397$
}

\begin{abstract}
Although the passive frame theory (PST) seems to be very plausible, the encapsulation of content generation deserves further elaboration. Many concepts and data considering the capacity theory, perception, and higher cognitive processes seem to contradict this principle. In general, PST can be suitable in many cases, but it needs to be completed by other mechanisms.
\end{abstract}

Despite the general structure of the passive frame theory (PST), which is well substantiated, the concept of encapsulation of content generation (sect. 4.1 tenet in target article) seems to be inconsistent with concepts and data from other domains in cognitive psychology. My commentary will challenge it from three directions: the capacity theory and physics, perception of illusions, and higher cognitive processes such as language and planning.

Taking up the same metaphor as Morsella et al. in the target article, consciousness may be represented as an interpreter (sect. 3.3, para. 4). In the real world, an interpreter can influence both people and the debate, albeit not voluntarily, by choosing one word instead of another or by using metalinguistic signs (i.e., tones, melodies, etc.) appropriate for the output language but different from the input one (cf. the concept of semantic incommensurability: Feyerabend 1975). From a perspective taken from physics, the interpreter introduces noise into the system as she is playing a role, as stated in Heisenberg's uncertainty principle. In cognition, every process requires and consumes cognitive resources, and a certain amount of error is associated with each transformation. Because any item in the cognitive system is susceptible of being modified by near items, why should the mechanism of consciousness not be prone to this principle? By assuming that errors increase under certain circumstances (i.e., high cognitive demands, high anxiety, low activation in drowsiness, etc.), contents may be affected (and modified) while they are in the conscious field, during and after the process of generation.

If we assume that inhibition of conscious contents is different from modification of contents, we can get a satisfactory outcome. Inhibition cannot be voluntarily applied to conscious contents (as discussed by Morsella et al.), but we cannot exclude that other contents or processes may modify the contents in the conscious field. Bottom-up influences could be excluded because the model clearly substantiates that they can generate content, but what about top-down influences?

The influence of past experience on perception (Duncker 1939) may be helpful. If a person looks at the picture in my Figure 1, she will perceive five segments. After turning her head $90^{\circ}$ to the left, the two letters $E$ and $F$ appear. From that moment on, it is not possible to perceive the segments anymore. The organized stimulus (i.e., the best guess made by the whole system, including both perception and past knowledge) hinders the initial perception in a permanent way. Along this view, Liu et al. (2012) have demonstrated that one's own "will" can influence the conscious perception of the direction of the spinning silhouette. Therefore, if separate items in the conscious field can interact in order to change the content, the principle of encapsulation can be applied to a very large subset of items, but not to every one. Phenomena like those observed due to the mirror neuron mechanism (Gallese et al. 1996) may support this assertion. If encapsulation were complete, it would be really difficult to explain the relevance of certain goal-directed actions, with respect to the same motor action without a goal. While representing a food object, it does not know whether it will be eaten or thrown away. However, our cognitive system does not represent a single item alone, if other information in the environment is tightly connected to the item itself (Iacoboni et al. 2005). If a grasping action is directed towards an apple, the system conveys the message that that action is going to be performed onto the apple, even though the apple is not present in the perceptual field but hidden behind a panel. Thus, task demands are not only activated by perceptual

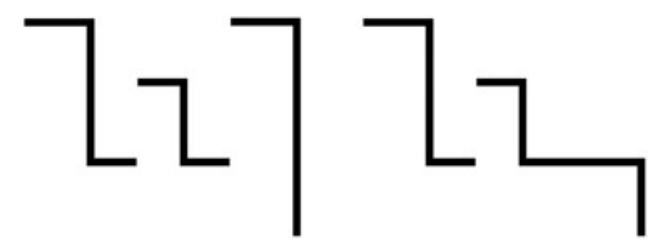

Figure 1 (Basso). Does this figure depict 5 segments or 2 letters? 
scenery, but also they can influence low-level processes like perception and attention.

Although it is widely accepted that desires and beliefs cannot modify these contents, Morsella et al. state that the same is true also for high-level processes. However, their example related to Thanksgiving dinner (sect. 5, para. 10) does not foster this assertion. What they termed as "language" is just another kind of (ready-made) action plan. The stimulus may trigger a movement that could be incompatible with drinking, but this is not language unless it involves syntax, semantics, and the generation of a planned utterance. Moreover, one person could have decided to move towards the cup of chocolate and to start talking at the same time. She stops talking only while drinking, but this is just execution of a well-known pattern of action embedded in the execution of an online speech, which needs a certain level of topdown control.

The experience of insight is perfectly in accordance with the predictions made by PST as it pops up in the conscious field without any (clear) awareness of its preparation. Many tasks, instead, call for an ordered series of actions in order to obtain the desired final state (i.e., planning). In the Travelling Salesperson problem, people need to plan the shortest path between a series of subgoals (MacGregor \& Ormerod 1996). Coherently with PST, it has been demonstrated (Basso et al. 2001; 2006) that people are aware of their intentions to change their plan during execution (due to conflicts between the initial plan and the ongoing situation). Because the number of intermediate subgoals does not affect the initial planning time, it was argued that the time before execution is used to determine the general strategy and the first movements. Thus, the actual strategy must be continuously determined during execution, and the constant planning index (Cazzato et al. 2010) justifies this assumption. Participants report to be aware of this ongoing process and that some tasks require additional effort. According to encapsulation, these participants were not able to refine their behavior during its execution, because they were aware of process outcomes only. The action-perception cycle is not involved, because planning could be made upon representations alone. Although planning may resemble mental rotation tasks, it plays a role in the approach-avoidance behavior (i.e., by determining the best path to escape), and it must be part of the behavioral repertoire of a "creature in the cave."

\section{What if consciousness has no function?}

\section{doi:10.1017/S0140525X15002010, e171}

\section{Susan Blackmore}

School of Psychology, University of Plymouth, Portland Square, Plymouth PL4 $8 A A$, United Kingdom.

susan.blackmore@plymouth.ac.uk

www.susanblackmore.uk

Abstract: An implicitly dualist or Cartesian materialist theory of consciousness is proposed without citing the many well-known problems with such theories. A function for consciousness is proposed with no reference to the possibility that "consciousness itself" has no function of its own. The theory builds on proposed "subset consensus" and "integration consensus" when in the literature there is no such consensus on these issues.

From the first sentence, the article by Morsella et al. assumes that consciousness has a function. The authors never consider the possibility that it may not and make many further assumptions that have frequently been challenged in the literature.

The question of function relates closely to what is probably the greatest gulf between theorists - whether consciousness is or is not something extra, or additional to, neural and biological processes. If it is, the hard problem (how subjective experiences arise from objective brain events; Chalmers 1995) is real, and consciousness must have evolved for a reason. If it is not, as in the case of the many variants of reductive or eliminative materialism, identity theory, and physicalism, then there is no hard problem, and consciousness evolved because perception, memory, learning, and other useful abilities evolved. Responding to the many wellknown thought experiments, only those who think consciousness is something extra believe that philosophical zombies are possible and that Mary the colour scientist learns something new when she emerges from her black-and-white room (Jackson 1982).

The authors are clearly in the first camp, not only with their concept of the "conscious field" and its contents, but also in their use of such phrases as "consciousness itself" (sect. 4.3, para. 2), the "regions responsible for consciousness" (sect. 3.4, para. 2), and brain regions or processes "associated with consciousness" (e.g., sect. 1.4, para. 2, and elsewhere in the target article). They reject Cartesian dualism (explicitly claiming to avoid the homunculus fallacy) but admit to retaining aspects of the Cartesian theatre. So their theory is a version of Cartesian materialism (Dennett 1991), as revealed in such phrases as "contents enter consciousness" (sect. 4.1, para. 2) and percepts and representations "become conscious" (sect. 3.5, para. 7).

These phrases imply that some processes, percepts, or representations are conscious while others are not, and the authors claim, as though it were uncontroversial, that there is a subset consensus on this issue. Citing Bleuler's (1924) textbook, they say, "When adopting a descriptive standpoint, even the most cursory examination of the brain reveals a contrast between conscious and unconscious processes" (sect. 1.2, para. 2). No, it does not. Examination with the naked eye reveals white and grey matter; with a microscope, glial cells, neurons, axons, and synapses; and with modern scanning techniques, further detail. Nowhere can we see what I have called the "magic difference" between conscious and unconscious regions or processes (Blackmore 2011). They claim that "the contrast between conscious and unconscious processes in the brain is somewhat inevitable" (sect. 1.2, para. 2). No. Not only is it not "inevitable," but also it is deeply problematic. What could it mean? That some brain processes have subjective experiences attached to them; that some give rise to consciousness; that the hard problem applies to only a subset? Indeed, saying that a brain process is "conscious" entails the mereological fallacy - that is, ascribing to part of an animal "an attribute which it makes sense to ascribe only to the animal as a whole" (Bennett \& Hacker 2003, p. 240). Morsella et al. are not alone in taking this view, and the hunt for the neural correlates of consciousness is based on the premise that such a distinction will be found, but so far there is no agreement about whether it has been or will be found.

The same can be said of the integration consensus. Claiming that the function of consciousness is integration, Morsella et al. contend that "consciousness serves an essential, integrative role for the somatic nervous system" (sect. 6, para. 5) and "consciousness integrates neural activities and information-processing structures that would otherwise be independent" (sect. 2.2, para. 1). I want to ask not only how subjective experience can be said to integrate objective activities and structures (implying a version of the hard problem or possibly some kind of unexplained downward causation), but also, more fundamentally, why these activities and structures need something extra to integrate them. Are they not integrated by structural connections or by the various processes proposed to account for binding (Cleeremans 2003)? Contrary to the authors' claim, there is no consensus over integration either.

The confusion is clearest in their discussion of global workspace theory (GWT). Morsella et al. cite more than 20 papers and books as evidence for this consensus but include in this list authors who hold totally opposed interpretations of GWT. These too can be divided into two main camps. On the one hand, there is a loosely Cartesian materialist or implicitly dualist account like the one proposed here. According to this, and the original formulation 
Commentary/Morsella et al.: Homing in on consciousness in the nervous system

by Baars (1988), items of information (or processes, percepts, thoughts, etc.) compete for access to the global workspace where they "become conscious" and are broadcast to the rest of the unconscious audience. This interpretation retains the hard problem and all of the difficulties of Cartesian materialism.

A completely different interpretation is more common (though not universal) in neuronal global workspace theory (Dehaene \& Naccache 2001) and means that no process or information has to "become conscious" or "enter consciousness." As Dehaene (2009) explains, brain-scale broadcasting creates the possibility of verbal and other types of report, and this is experienced as a conscious state. There is no additional consciousness or subjectivity. This is what Dennett (2005), more colourfully, calls "fame in the brain" or "cerebral celebrity." Fame is not something additional to being widely known, nor is consciousness something additional to being widely broadcast. In this interpretation of GWT, there is no dualism or hard problem. These views are deeply opposed, and there is no consensus as to which is right.

In summary, the article by Morsella et al. proposes an implicitly dualist or Cartesian materialist theory of consciousness without citing the many well-known problems with such theories and claiming consensus where none exists. Their discussion of olfaction is a helpful and interesting addition to our understanding, but their theory of the function of consciousness does not stand up to scrutiny.

\section{Consciousness around the time of saccadic eye movements}

\section{doi:10.1017/S0140525X15002022, e172}

\section{Bruce Bridgeman}

Department of Psychology, University of California Santa Cruz, Santa Cruz, CA 95064.

bruceb@ucsc.edu

http://people.ucsc.edu/ bruceb/

Abstract: During saccadic eye movements, the job of the nervous system is not to perceive a change in stimulation from the receptors, but to prevent a change from reaching consciousness. These movements occupy an intermediate range between actions that are almost always conscious, such as finger movements, and those that are never conscious, such as the smooth-muscle actions of the digestive system. Consciousness in this context requires attention to the consequences of an action.

In their motor-oriented approach to consciousness, Morsella et al. distinguish between conscious and unconscious processes: "Although one is unconscious of these complex programs (Johnson \& Haggard 2005), one is often aware of their proprioceptive and perceptual consequences" (target article, sect. 3.1, para. 2, emphasis in the original).

I will discuss a case in which perceptual consequences remain hidden despite large changes in sensory stimulation. The situation is the most frequent of all human behaviors, the saccadic (jumping) eye movement that moves visual fixation from one location to another. Saccadic eye movements occupy an intermediate range between actions that are almost always conscious, such as movements of the fingers, and those that are never conscious, such as the smooth-muscle actions of the digestive system. Like breathing, the generation of eye movements can be conscious but normally is not. As a striated muscle system, the movements should be conscious in the context of Morsella et al.'s elemental, action-based, simple, and evolutionary-based (EASE) perspective. Consciousness requires attention to the consequences of an action.

Here the job of the nervous system is not to perceive a large change in stimulation from the receptors, but to prevent such a change from reaching consciousness. All visual information arrives from retinas that encounter a large change in stimulation, a shift of the entire visual world, with every saccade. Normally the change is not perceived, but under some circumstances, it can be perceived (Deubel et al. 1998). If a saccade causes gaze to reach its target, the result is usually space constancy-negatively defined, this means that a jump of the retinal world is not perceived despite the large change in the stimulus. The motor-oriented approach of Morsella et al. links the sensory and motor aspects of a saccade, allowing a reorientation in terms of reafference.

Retinal signals reach the primary visual cortex and beyond in a code that is not space-constant-cortical stimulation shifts with eye movements even though perception does not (Bridgeman 1973). Hence, we already know that the content of the primary visual cortex is not conscious. The paradoxical situation arises that the proprioceptive and perceptual consequences of saccades reflect the world, that does not move, and not the retinal image that does move. In fact, there is no visual brain area that shows no change across a saccadic eye movement, because space constancy is in this sense a systematic failure of conscious perception rather than a detection of an external event. Consciousness is directed to the stable world, not to the moving receptors.

\section{An "ecological" action-based synthesis}

\author{
doi:10.1017/S0140525X15002046, e173
}

\author{
Jonathan de Vries ${ }^{\mathrm{a}, \mathrm{b}}$ and Lawrence M. Ward ${ }^{\mathrm{b}, \mathrm{c}}$ \\ aInterdisciplinary Studies Graduate Program, University of British Columbia, \\ Vancouver, British Columbia, V6T1Z4, Canada; ${ }^{\mathrm{b}}$ Department of Psychology, \\ University of British Columbia, Vancouver, British Columbia, V6T1Z4, Canada; \\ ${ }^{\circ}$ Brain Research Centre, University of British Columbia, Vancouver, British \\ Columbia, V6T1Z4, Canada. \\ Iward@psych.ubc.ca devriesj@alumni.ubc.ca \\ http://www2.psych.ubc.ca/ /ward/
}

Abstract: We expand upon Morsella et al.'s synthesis in the direction of what Gibson (1979) called an ecological approach to perception. Morsella et al. describe consciousness as a director of voluntary action, but they understate the role of the environment in its evolution as well as in directing behavior. We elaborate these roles in the context of the concept of affordances.

Why do we experience such a rich visual world all of the time our eyes are open, but seem to be aware of smells only when they first occur? There do seem to be more than incidental and irrelevant differences between the olfactory system and the other four major sensory systems. Morsella et al. suggest that we study the olfactory system for clues as to the neural correlates of conscious perceptual content. However, the olfactory system is admittedly a very primitive, albeit highly conserved, sensory system. The olfactory cortex is paleocortex, only three layers deep. Moreover, as Morsella et al. emphasize, olfactory information does not pass through a thalamic "relay" nucleus on its way to the olfactory cortex, but rather goes directly there from the olfactory bulb, only informing the mediodorsal nucleus of the thalamus after paleocortical processing. Even that pathway is minor, as olfactory input is mainly directed to the orbitofrontal cortex, from where it also projects to the mediodorsal thalamus. Although some insights about consciousness can surely be gained by taking the olfactory system as a model, we believe that it is sufficiently different from the other sensory/perceptual systems that those insights will not be sufficient to explain much about the more sophisticated, and recently evolved, systems that subserve mammalian consciousness. Indeed, there might even be two separate types of consciousness: paleoconsciousness (paleoC), arising from a phylogenetically old system like the olfactory system, and neoconsciousness (neoC), arising from a more recent adaptation involving thalamic relay nuclei and extensive neocortical processing. 
In the service of describing the newer type of consciousness, we offer the following observation: neoC has many properties that paleoC does not have. Most compelling to us is the fact that as long as we are awake and have our eyes open, we experience a rich visual scene. Conflict-resolving contents don't spring into and out of our consciousness, as described for Morsella et al.'s cave-dweller, although they do spring into and out of attention focus. Rather, we experience a wide range of action affordances; that is, following Gibson (e.g., 1979), Norman (e.g., 2013), Turvey (e.g., 2015), and others, we experience action possibilities. The environment affords many such possibilities, the vast majority of which will not be relevant to current goals and plans, although they potentially could be. So why do we have perceptual and cognitive systems that afford such a huge set of possible actions, most of which will never be taken, conflict or no? Why did a simple system (paleoC), as described by Morsella et al., not suffice, in which relevant, conflict-resolving, perceptual content pops into consciousness when needed?

We contend that the more elaborate system (neoC) evolved, along with neocortex, to extend the range of affordances of animals in the service of niche construction (Withagen \& van Wermeskerken 2010). We suggest this is true at least for animals that appeared in the past several hundred million years, especially mammals and birds, which share most features of thalamocortical circuitry (Butler 2008). Niche construction theory views affordances as coevolving along with the various species' physical characteristics to allow adaptive behavior in new niches that themselves are partially constructed from the new affordances available.

An especially important feature of the neoC system, which we contend does indeed involve essential thalamocortical circuitry, is that the affordances it provides allow for some very sophisticated behaviors. Many of these involve moving the entire body in complicated ways (e.g., a gymnast engaged in a floor routine). Another set enables us to extend our behaviors into nonlocal environments (cognitive maps; Tolman 1948) that allow for planning whole body movements to places beyond the range of direct perception (though see Stepp \& Turvey [2015] and Turvey [2015] for alternatives).

It should be noted that the behavior of Morsella et al.'s "creature in the cave" could arguably be construed as either conscious or non-conscious. For instance, the experience of "acting involuntarily" (e.g., suddenly reaching out to catch a falling object, the motions one's body takes to recover/brace during a fall, etc.) may only lack the illusion of voluntary action because the action is required to occur before its stimulus can reach consciousness (cf. Glover 2004). Whether this issue can be resolved (re neoC/ paleoC) may require further research, though determining "refresh rates" in other animals may prove fruitful.

Finally, we wish to point out that the paleoC and neoC systems, in advanced animals that have both, must work together to direct action. The olfactory system does project to neocortical areas, and those project their more advanced outputs to the mediodorsal thalamus, presumably integrating there with the information coming in directly from the piriform cortex. In addition, olfactory information entering the orbitofrontal cortex is integrated with information from every other sensory/perceptual system, and the integrated information is projected back to the sending areas, including the thalamus. It is therefore not surprising that understanding olfactory consciousness is difficult; the paleoC system does not normally function in isolation in advanced animals. When parts of the newer system to which it is connected are knocked out, however, as in damage to the mediodorsal thalamus or orbitofrontal cortex, anomalies of olfactory consciousness are experienced. One such anomaly is so-called blindsmell, in which a person denies olfactory experience but can make some olfactory discriminations. Notably, Sobel et al. (1999) observed increased activation levels of the orbitofrontal cortex and thalamus during high (compared with low, "non-perceptible") concentrations of odorants. This phenomenon, and its brain correlates, closely resembles "blindsight," "blindhearing," and "blindtouch," in all of which apparently unconscious information processing helps to guide action (Zucco et al. 2014).

It is possible that our two suggested systems, paleoC and neoC, could share some fundamental mechanism that gives rise to experience. It is also possible that there is more than one such mechanism, with the more complicated one absorbing the less complicated, except when the more complicated one itself is incapacitated. Further exploration of the similarities and differences between olfactory consciousness and that illustrated by vision, hearing (especially speech), touch, and taste should continue to yield insight into this mystery.

\section{Calling for a developmental perspective on action-based consciousness}

\author{
doi:10.1017/S0140525X15002034, e174
}

\author{
Hana D'Souza ${ }^{\mathrm{a}, \mathrm{b}}$ and Andrew J. Bremner ${ }^{\mathrm{a}}$ \\ asensorimotor Development Research Unit, Department of Psychology, \\ Goldsmiths, University of London, London SE14 6NW, United Kingdom; \\ ${ }^{\mathrm{b}}$ Centre for Brain and Cognitive Development, Birkbeck, University of London, \\ London WC1E 7HX, United Kingdom. \\ hana.dsouza@gmail.com a.bremner@gold.ac.uk \\ http://www.cbcd.bbk.ac.uk/people \\ http://www.gold.ac.uk/psychology/staff/bremner/
}

Abstract: Human newborns can resolve some response conflicts in order to adapt their behaviour, suggesting that the newborn has consciousness according to Morsella et al.'s framework. However, we pose a range of developmental questions regarding Morsella et al.'s account, especially concerning the role of consciousness in the development of action.

Morsella et al. argue that consciousness comprises not higherorder cognitive processes, but basic sensory components necessary for purposeful action. Whereas the development of higher-order cognitive processes remains a matter of debate (e.g., Blakemore \& Choudhury 2006; Zelazo 2004), it is more accepted that the sensory constituents of action change substantially in early life as a result of the interplay between sensorimotor experience and brain development (Gibson \& Pick 2000; Mareschal et al. 2007; Thelen \& Smith 1996). Although it is unclear whether Morsella et al. view consciousness as a static or developmental phenomenon, all accounts of psychological functioning including those concerning consciousness are necessarily constrained by what we know of the developing organism. Hence, we believe that a consideration of development provides an important point of reference and validation for Morsella et al.'s proposal.

Morsella et al. propose that the function of consciousness is to allow the integration of intentional drives towards certain skeletal muscle outputs which are incompatible in a given situation. One example which they offer is that of holding one's breath underwater-they view consciousness as a field in which the conflicting goals of being underwater and the natural tendency to breathe are resolved. In their explanation of this position, Morsella et al. introduce "a hypothetical, simplified, human-like mammal that, though conscious [...], is not equipped with many of the complicated abilities/states associated with adult humans" (sect. 1.3, para. 1, italics added). A non-hypothetical organism already fits Morsella et al.'s description: the human newborn.

We want Morsella et al. to replace their hypothetical "creature in the cave" with a human newborn for two reasons. First, this will allow us to examine whether their framework can describe real examples. Consciousness is likely to have a specific biological substrate working under different constraints to those of the "creature in the cave". Second, a consideration of developmental differences between newborn and (let's say) adult, and also the processes of development, will help test and extend their proposal. 
For instance, we can ask whether a human newborn automatically reacts to sensations one at a time or whether it is able to resolve response conflict for purposeful action. If it merely reacts to consecutive sensations, then one might argue that, according to Morsella et al.'s perspective, the newborn has no need for a conscious field, and thus consciousness may emerge gradually over development. However, as we shall see, newborns can resolve some response conflicts in order to adapt their behaviour.

Although the newborn infant's ability to generate voluntary action is relatively limited, neonatal reflexes (Prechtl 1993), traditionally viewed as automatic (outside-of-consciousness) stimulus reactions, have been argued by some to represent intentional actions (e.g., Rochat 2001; von Hofsten 2007). Therefore, we can ask whether behavioural reflexes in newborns can be modulated by competing intentions. The sucking reflex is activated whenever the nipple touches the back of the palate. This results in the jaw movements needed to induce milk flow (Prechtl 1993). In fact, this behaviour is not completely reflexive; newborns can alter their sucking behaviour in response to the taste of the liquid (e.g., Crook 1978). Salt solutions lead to shorter bursts of sucking in newborns, relative to distilled water. Morsella et al.'s conscious field permits contents about touch and taste to integrate and influence action, resulting in infants modifying their sucking behaviour. Morsella et al.'s framework thus seems to indicate that human newborns are conscious.

Although newborns can modulate their sucking action in response to the salinity of the liquid, there have nonetheless been cases of accidental poisoning through excessive sodium intake following mistakes in food preparation (e.g., Finberg et al. 1963). It seems that, in newborns, taste can only modify (not fully suppress) the sucking response, leading to continued ingestion of food even when distasteful. Nonetheless, infants are unlikely to be presented with dangerous liquid, so their sucking response is adaptively stronger than their reaction to the salinity of liquid. However, adults have better control over food intake: they are able to spit out distasteful food. Although both infant and adult behaviours are adaptive, we wonder whether the differences are due to developmental changes in consciousness. If they are, then an account of what changes in consciousness, and how it changes, is needed.

Morsella et al. propose that consciousness functions to provide a structure in which conflicting purposeful movements can be resolved. As such, one can ask how the realisation of this function might change over development. Certainly, the relevant movement conflicts change, and it takes time for infants to develop a well-structured behavioural repertoire that could form the mature "content" of Morsella et al.'s "conscious field." To take an example, months before infants are able to reach purposefully for an object, they spontaneously flap their arms when presented with it (Bhat \& Galloway 2006). Over development, these spontaneous flapping movements come to more frequently contact objects, eventually leading to proficient goal-directed reaching. Considerable time is involved in these developments: Although in one waking hour the average toddler takes 2,368 steps, which is the equivalent length of 7.7 American football fields (Adolph et al. 2012), it is still several years before the movements they select in walking become adultlike (Adolph \& Robinson 2015). What kind of role does Morsella et al.'s conscious field play in the development of competent purposeful action selection? In the example given above, do young infants' experiences of the conflict between emerging representations of "spontaneous flapping" and "approaching an object" drive the development of proficient reaching?

To sum up our line of questioning, we wonder to what extent the conscious field is required for development or is a result of development itself. Indeed, we had many questions concerning why Morsella et al. felt that consciousness was necessary to resolve the kinds of conflicts which the "creature in the cave" and the human newborn face. It might be that a developmental perspective could provide an answer to such queries, by highlighting the need for an ontogenetic mechanism by which we first come to resolve conflicts.

\section{Getting back from the basics: What is the role for attention and fronto-parietal circuits in consciousness?}

\author{
doi:10.1017/S0140525X15002058, e175
}

Paul E. Dux

School of Psychology, The University of Queensland, St. Lucia, Queensland 4072, Australia.

paul.e.dux@gmail.com p.dux@psy.uq.edu.au

www.paulduxlab.org

Abstract: Morsella et al. present a novel theory of consciousness which is more "low-level, circumscribed, counterintuitive, and passive" than previous accounts. There is much to like about this approach. However, what are the explanatory costs associated with these characteristics? In particular, how does this low-level and passive theory accommodate evidence for top-down attention being necessary (but not sufficient) for visual consciousness?

Much of science is reductionist, whereby methodology and theory are expressed in ever-smaller and fine-grained entities in order to investigate and characterize a phenomenon or process of interest (Koch 2012). In the target article Morsella et al. apply this method and introduce a provocative conceptual framework to advance our understanding of consciousness. Specifically, they argue that in order to study this most elusive of neural processes, one should look at it in its most basic form. Indeed, they focus on a hypothetical, simplified human-like mammal that, although conscious, does not possess high-level abilities associated with human information-processing - for example, indirect cognitive control. In addition, they study the olfactory system, as opposed to the more complex visual system which has been the focus of previous consciousness research (Crick \& Koch 2003). I commend the authors for taking on such a daunting problem, their thorough analysis of the relevant literature, and their novel approach; however, the basic framework Morsella et al. present may be limited it terms of its explanatory power. Indeed, it appears unable to account for a wide array of literature which shows that, in the visual domain (humans' dominant sense), higher-level cognitive processes, such as top-down attention and re-entrant processing in the brain - whereby frontal-parietal regions project back to sensory areas - play a vital role in consciousness.

A recent trend in the empirical literature has been to argue that conscious and top-down attention are distinct and do not share a causal relationship. Put differently, attention can be devoted to unconscious stimuli, and consciousness can be achieved without a stimulus being the subject of attention (Koch \& Tsuchiya 2007). However, as elegantly argued by Cohen et al. (2012), there is little evidence for this. Specifically, attention has to be able to be deployed to unconscious stimuli, otherwise how would these items reach consciousness? Having said this, attention is not all or none, and consequently, just because an item is attended does not guarantee that it will enter awareness (thus, attention is necessary but not sufficient). In addition, Cohen et al. provide a thorough review of the literature and demonstrate that previous findings reporting properties of the environment (e.g., gist of a scene; Mack \& Rock 1998) entering awareness without attention are flawed and do not hold up to dual-task attentional manipulations (Cohen et al. 2011). In short, there is strong evidence that top-down attention and consciousness are inherently linked. Given that top-down attention is capacity limited/effortful, this suggests that consciousness is not entirely passive and that it relies on higher-level goal-related information processing (not possessed by the creature in the cave).

Evidence from neuroscience also suggests that visual consciousness draws on high-level resources and that the neural basis for consciousness goes beyond subcortical regions. Arguably the most heavily used phenomenon for studying visual consciousness is the attentional blink $(\mathrm{AB})$ : This refers to subjects' typically impaired performance in reporting the second of two targets in a rapid serial 
visual presentation stream if it appears within 200-500 msec of the first target relative to when it appears outside this window (Raymond et al. 1992). The AB is thought to reflect a failure of perceptual awareness, where the missed second target does not enter consciousness. Considerable functional imaging work has investigated the neural substrates of this phenomenon and localized it to frontal-parietal regions. For example, Marois et al. (2004) found, using a dual-target Rapid Serial Visual Presentation (RSVP) task with scene stimuli for target 2 , that when this item was missed, and it failed to enter awareness, it still activated the parahippocampal place, whereas the lateral prefrontal cortex did not respond to missed second targets. Similarly, research on object substitution masking (OSM; Enns \& Di Lollo 1997), where a sparse (e.g., four-dot), temporally trailing mask obscures the visibility of a briefly presented target, also implicates top-down processing. Indeed, Hirose et al. (2007) observed that disruption of visual area MT (middle temporal/V5) via repetitive transcranial magnetic stimulation (rTMS) influenced the magnitude of OSM. This is consistent with the re-entrant processing account of OSM offered by Di Lollo et al. (2000) who argue that the phenomenon represents iterative hypothesis testing with anterior (e.g., frontal) regions sampling high-resolution sensory information from posterior visual areas via feedback. Finally, recent work by Zhang et al. (2011) has shown that even the inter-ocular fluctuations in consciousness that are observed during binocular rivalry (BR), which were previously thought to be automatic and spontaneous, are dependent on top-down attention. These authors employed electroencephalogram (EEG) frequency tagging via steady-state visual evoked potentials to show that stimulus representations stopped rivaling when attention was diverted away from the rivalry display. Collectively, these results using the prominent visual consciousness phenomena of the $\mathrm{AB}$, OSM, and BR, all show that top-down attention processing plays a vital role in consciousness. In addition, when considering that topdown attention has been shown to tap frontal-parietal cortical circuits (see Corbetta et al. 2008), this suggests that consciousness is, at least partially, both high level and active rather than passive.

So where does this leave us with the passive frame theory offered by Morsella et al.? It is clear that theirs is an internally consistent framework that offers a unique perspective and impressive account of the most basic form of consciousness. However, the challenge of characterizing higher levels of consciousness (although $\mathrm{AB}, \mathrm{OSM}$, and BR are thought to be perceptual phenomena) in a species that leads a richer and more challenging life and that has more sophisticated and complex cognitive hardware, remains to be addressed. It may be the case that passive frame theory could be extended to accommodate a broader range of findings and phenomena in humans; however, this would require significant revision. It must also be noted that such a revision is necessary if this theory is to provide a comprehensive framework that facilitates the interpretation of consciousness data. Having said this, Morsella et al. are not alone here, and indeed the difficulty of marrying reductionism and generalizability is one that all of us working in this field face.

\section{How does consciousness for action relate to attention for action?}

\section{doi:10.1017/S0140525X1500206X, e176}

\section{Elizabeth A. Franz}

Department of Psychology, University of Otago, Dunedin 9016, New Zealand. Ifranz@psy.otago.ac.nz

http://www.otago.ac.nz/psychology/research/otago028082.html

Abstract: The interesting target article by Morsella et al. addresses critical issues that impact our understanding of consciousness. It is surprising, however, to see no treatment of the relationship between attention and consciousness, particularly given available models. Whether olfaction is most suitable as a model system to study consciousness for action also seems questionable. These issues are elaborated in the present commentary.

The article by Morsella et al. is an interesting and thought-provoking review. Two primary issues kept coming to mind during my reading, both of which seem critical to address. The first is that the review avoids mentioning anything about the relationship between attention and consciousness. The second concerns whether the olfactory system in all of its simplicity is suitable as a model system to study consciousness for action (particularly in humans).

Cited in the target article but not elaborated, Keller (2011) emphasizes the relation between attention and consciousness when considering olfactory consciousness. In a similar vein, work in my lab has considered the link between consciousness and attention; however, the focus is not on olfaction, but on conflict situations between the limbs (bimanual actions) in an allocation of attention to action (AAA) framework (Franz 2012). As in the target article by Morsella et al., the AAA framework was inspired by the earlier writings of Sperry (1952) and James (1890/1918), and a major tenet is ideomotor theory (Hommel 2010). Thus, the similarities across approaches are quite remarkable.

Higher levels of the 3-level AAA framework incorporate examples of bimanual conflict in actions such as drawing a circle and a line, or circle and square (Franz 1997; 2003; 2012; Franz \& Ramachandran 1998; Franz et al. 1991; 1996), or reaching with one hand to a far target and one hand to a near target (Franz \& McCormick 2010; Kelso et al. 1979). The "empirical proof" of integration (of lower and higher levels of processing) comes from additional examples in which the two hands produce a unified action rather than conflicting actions as a result of the presence of visual perceptual features or specific verbal instructional commands (Franz \& McCormick 2010; Franz et al. 2000; 2001); this propensity to unify information at the highest level possible is proposed as a fundamental property of the "economical brain" (Franz 2010; 2012; Franz \& McCormick 2010). Higher levels are further discussed in terms of automatic or conscious processes of activation and inhibition (level 2: AAA), and action selection (level 3: AAA), with all levels implicating a mix of conscious and unconscious processing.

Consider a specific point of overlap found in the target article, which states that "there must be a (unconscious) mechanism by which one action plan can influence behavior more than other activated action plans" (sect. 3.3, para. 3). AAA argues the same, building on an action selection model (Mink 1996) in which activation of an action plan occurs from among other prepotent action plans that must be inhibited (akin to action options). This process implicates basal-ganglia (striatal) and thalamic-cortical (primarily frontal) circuits as being key (Franz 2006; 2012; Mink 1996) and can occur automatically through activation functions.

The target article focuses primarily on what most resembles the lowest level of AAA. In level 1 of AAA (see Figure 1 in Franz 2012), primarily exogenous inputs capture awareness of an organism transiently (and automatically), leading eventually to trial-anderror learning which results in formation of a perceptual-action code akin to ideomotor theory (Hommel 2010); recent experimental support comes from studies using evoked response potentials (Bednark et al. 2013; Bednark \& Franz 2014).

Three differences are clear across frameworks: (1) the focus on attention and its relation to consciousness (Franz 2012; Keller 2011) not present in Morsella et al.; (2) the focus on "in the service of action" (Franz 2012; Morsella et al.) not present in Keller (2011); and (3) the focus on olfaction (Keller 2011; Morsella et al.) not present in Franz (2012).

Furthermore, the work of Grossberg, although briefly cited, seems far more relevant than suggested in the target article. Grossberg (1999) addresses the question of "how an intelligent being can autonomously adapt to a changing world" (p. 4, emphasis in original). Grossberg (1999) suggests that top-down expectation selectively amplifies some features of bottom-up input while suppressing others, and bottom-up signals reactivate those same top-down expectations (p. 10). This reactivation leads to a resonant state which 
Commentary/Morsella et al.: Homing in on consciousness in the nervous system

is necessary for the brain to achieve consciousness (a conscious percept). Without it, there is no conscious percept. Furthermore, Grossberg (1999) suggests that a form of automatic attention can operate at very low levels of processing (p. 32). Perhaps this also suggests that the distinction between "passive" and "active" becomes rather blurry within the context of a process model?

According to AAA, increasingly embedded levels of processing are built upon a basic sensorimotor system as a result of evolved adaptations. This enables representation (possibly similar to conscious content), and re-representation in a hierarchical neural system, which has evolved through embodied actions, consistent with the very early views of John Hughlings Jackson (1884; cf. Franz \& Gillett 2011; Gillett \& Franz 2013). A statement in section 5 of the target article seems to capture the essence of this idea: "All influences on skeletomotor behavior, from the highest-level processes (e.g., language) to the lowest-level processes (e.g., pain withdrawal), must engage the skeletomotor output system" (para. 4).

If olfaction is being proposed as a model system for studying consciousness, and consciousness is for action, then what about the implied olfaction for action link? The skeletal action system is highly adaptive with growth of the organism (the limbs increase in size with age), and is controlled by numerous cognitive and perceptual processes together with influences of a dynamically changing environment (Franz 2012; Grossberg 1999). Limb systems respond with fast simple reaction times to visual and auditory stimuli (often between 200 and 300 milliseconds; Greenwald 1972; Hughes \& Franz 2007; Shen \& Franz 2005; Tassinari \& Berlucchi 1993). Visual stimuli are experienced as uninterrupted, whereas olfactory experience is discontinuous due to sniffs occurring with gaps between them (1.6 seconds is cited as the gap; Laing 1983; Mainland \& Sobel 2006; cited in Keller 2011). Thus, olfactory experience is temporally unstructured, and olfaction is essentially a slower sense (Keller 2011). Gottfried and Dolan (2003) report a mean olfactory reaction time of 1.539 seconds, and further elaborate that "the nose smells what the eye sees" (p. 375), that is, odor perception is largely affected by interactions with vision. Is an olfaction to action link all that consistent with available evidence?

In sum, while I have no reason to disagree with the suggestion that olfaction is an ideal system to study consciousness, I am less convinced that olfaction is a suitable model system for an approach aimed at informing our understanding of "consciousness for action."

\section{Consciousness of emotions and action selection}

\author{
doi:10.1017/S0140525X15002071, e177
}

\author{
Guido Gainotti ${ }^{\mathrm{a}, \mathrm{b}}$ \\ ${ }^{a}$ Center for Neuropsychological Research, Institute of Neurology of the \\ Policlinico Gemelli/Catholic University of Rome, 00168 Rome, Italy; \\ ${ }^{\mathrm{b}}$ Department of Clinical and Behavioral Neurology, IRCCS Fondazione Santa \\ Lucia, 00142 Rome, Italy. \\ gainotti@rm.unicatt.it
}

Abstract: The domain of emotions corresponds to the consciousness model proposed by Morsella et al. The action schemata unconsciously activated by spontaneous emotions are indeed automatically selected from a small number of innate operative patterns, whereas behavioral responses selected at the cognitive level of emotional processing consist of strategic plans, leading to a conscious integration of goal-directed actions.

In their target article "Homing in on Consciousness in the Nervous System: An Action-Based Synthesis," Morsella et al. propose the passive frame theory, an internally coherent framework that, from an action-based perspective, assumes that the primary function of consciousness consists in serving as a frame that reflects a form of integration associated with action selection, which directs skeletal muscle output, thereby yielding adaptive behavior. A behavioral domain which strongly corresponds to this model is the domain of emotions. Emotions are, indeed, considered as adaptive behaviors, capable of interrupting with an urgency procedure the ongoing action, to rapidly select a new operative scheme (Oatley \& Johnson-Laird 1987). The action schemata, unconsciously activated by the process of spontaneous emotional computation, can be viewed as action dispositions, automatically selected from a small number of innate operative patterns, corresponding to the basic emotions and preparatory for (or supporting) specific behavioral responses (Frijda 1986). These behavioral patterns help solve important adaptive problems of the human species, and include expressive-communicative components, postural changes, locomotion, bodily movements, and a sizeable recruitment of the autonomic nervous system. On the other hand, behavioral responses selected by the cognitive component of the emotional system consist of controlled strategic plans, which do not include a communicative-expressive component and a concomitant strong activation of the autonomic nervous system, but an integration of actions aiming to find the most appropriate solution to the adaptive problem.

This difference between spontaneous and cognitive levels of emotions is due to the fact that, according to most authors, emotions have a complex hierarchical structure, in which some sensorimotor aspects are unconscious, but other aspects, such as the subjective feelings and the selection of the skeletal muscle output, are conscious. In particular, Leventhal (1987) proposes that human emotions may be based on three functional levels: (a) the sensorimotor, (b) the schematic, and (c) the conceptual level. The sensori-motor level consists of a set of innate, universal expressivemotor programs, which are triggered automatically by a certain number of stimuli and include components of motor and vegetative activation, as well as the corresponding subjective emotional feelings. During individual development, these basic programs are linked, by mechanisms of conditioned learning, to situations of the individual experience, building the "emotional schemata," which correspond to partly unconscious spontaneous emotions, are automatically elicited, and are accompanied by subjective feelings which constitute the hallmarks of a true emotion. The last stage of this model is the conceptual level, which is based on mechanisms of conscious declarative memory and does not store instances of concrete emotional experiences, but abstract notions about emotions and the social rules that allow their expression, leading to the conscious and controlled selection of the most appropriate action schemata.

A further difference between the above-mentioned levels of emotional processing is that (according to the clinical and experimental data reviewed in Gainotti [2012]) unconscious aspects of emotions are mainly subsumed by subcortical structures, whereas the more conscious aspects are supported by cortical structures. Furthermore, a right hemisphere network mainly subsumes spontaneous emotions, whereas a left hemisphere neural system, related to language and to the control of intentional movements, supports the cognitive aspects of emotions. Therefore, contrary to what happens in spontaneous, automatic forms of emotional behavior (in which vegetative, expressive, and postural components of the response are unconsciously produced), in controlled and intentional forms of emotional expression conscious experience is accompanied by a selective and deliberate action selection, which directs skeletal muscle output towards goal-directed patterns of response.

\section{Insights on consciousness from taste memory research}

doi:10.1017/S0140525X15002083, e178

\section{Milagros Gallo}

Department of Psychobiology, Institute of Neurosciences, Center for Biomedical Research (CIBM), University of Granada, 18016 Armilla, Granada, Spain.

mgallo@ugr.es

http://ineurociencias.ugr.es/pages/personal 
Abstract: Taste research in rodents supports the relevance of memory in order to determine the content of consciousness by modifying both taste perception and later action. Associated with this issue is the fact that taste and visual modalities share anatomical circuits traditionally related to conscious memory. This challenges the view of taste memory as a type of non-declarative unconscious memory.

I consider most relevant the challenge that Morsella et al.'s proposal poses to the traditional account of consciousness in terms of higher cognitive/executive processes, which is based mainly on visual research data. Among the various issues their target article raises, the focus on the olfactory system merits attention, given the general misconception about the low cognitive level of chemosensory modalities such as olfaction and taste. Because a similar picture is found with respect to taste, I consider it relevant to draw the authors' attention to the gustatory modality as a valuable case for the theory, because taste perception is closely linked to action. Except in laboratory settings where taste solutions can be applied to unconscious subjects, tasting under normal circumstances requires voluntary ingestion. Thus, taste research in rodents can shed light on the following issues.

First, the role of memory in modifying the percept which leads to action seems to be dismissed in the theory. When speaking about motivation, Morsella et al. mention the role of memory as part of general higher-order cognitive processes with little influence over perceptual processes. In fact, they state that "the [conscious] field itself has no memory ... it only presents, for action systems, the outputs of dedicated memory systems" (sect. 6, para. 2). However, even in the "creature in the cave" metaphor the conflict arises from memory, because the smell of smoke has necessarily been previously associated with danger in order to induce the urge to exit. It can be said that memory is in the field of consciousness even if it is not conscious. Thus, memory has presumably modified the smell of smoke at the lower level of sensory processing. Likewise, the gustatory system is modified by learning and memory even at the lower sensory relay levels in the brainstem (Yamamoto \& Yasoshima 2007). Thereafter, it can be proposed that the conscious content of a food item necessarily includes its hedonic value that in turn depends on memory. A taste is novel, safe, or aversive but never neutral, because tasting implies ingesting and this is followed by unavoidable consequences. The outcome can be either positive, such as to relieve thirst and/or hunger, or negative, such as to experience visceral malaise (see Gallo \& Rolls [2012] for a special issue on the topic). According to the authors' proposal, for the conscious field to be complete and unambiguous during action selection, it would not be adaptive for a taste to be represented without its hedonic features, just as it would not be adaptive for an object to be represented in the wrong spatial location.

Second, animal research on taste memory is consistent with the authors' proposal, which locates consciousness at a lower level of the nervous function than previously proposed. For example, in the anatomically based dichotomy between declarative and nondeclarative long-term memory (Squire 2004), consciousness has been traditionally associated with declarative memory (also termed explicit and cognitive memory). This conscious memory is thought to be mediated by a medial temporal lobe system involving the hippocampus and the adjacent perirhinal, entorhinal, and parahippocampal cortex, as well as connections with other cortical areas. Visual and taste recognition memory tasks in rats have been conventionally viewed as paradigms of declarative and non-declarative memory, respectively. The primitive phylogenetic and ontogenetic origin of the gustatory system might have contributed to this state of affairs. Taste recognition memory represents a basic adaptive mechanism. The term refers to the ability to assess the familiarity of a taste that was not followed by negative consequences in previous encounters. Concomitantly with the taste being classified as safe, consumption increases, thus showing attenuation of neophobia. Research evidence using recognition memory tasks suggests that taste and visual memories share an anatomical basis. In fact, consolidation of safe taste memories has been associated with the hippocampus and the perirhinal cortex (De la Cruz et al. 2008). Also, we have assessed Foslike immunoreactivity to demonstrate amygdala-dependent changes in the activity pattern of the perirhinal cortex during the formation of taste memory (Gómez-Chacón et al. 2012; 2015). This indicates that taste recognition memory involves some of the areas previously related to visual recognition memory. Therefore, it seems that the strict anatomically based dichotomy of declarative versus non-declarative memory applied to visual and taste recognition memory may need to be updated when exploring memory maintenance mechanisms because independent circuits do not function in isolation from each other and may share common components.

Furthermore, in visual recognition memory, there are data supporting the relevance of neural circuits that involve lower brain areas, such as the thalamus. In fact, recollection and familiarity, proposed as different components of recognition memory, seem to depend on dissociable brain circuits. Recollection involves remembering specific contextual details about a prior episode, whereas familiarity involves simply knowing that an item was presented, without having available any additional information about the learning episode (Squire et al. 2007). In addition to the functional dissociation of the temporal lobe circuits that attributes to the hippocampus and the perirhinal cortex selective roles in recollection and familiarity, respectively (Aggleton \& Brown 2006), the neural circuits proposed include, among other subcortical areas, the anterior and the mediodorsal thalamic nuclei, respectively (Carlesimo et al. 2015).

In all, it can be proposed that the authors' theory would benefit from reconsidering the role of memory, whether available to consciousness or not, because it modifies the encapsulated perceptual contents themselves. Taste memory is a good model to be taken into account.

\section{ACKNOWLEDGMENT}

The author is grateful for grant PSI2014-57643-P (MINECO, Spain), supported by FEDER funding.

\section{Consciousness weaves our internal view of the outside world}

doi:10.1017/S0140525X15002095, e179

\author{
Moshe Gur \\ Department of Biomedical Engineering, Technion, Haifa, 32000, Israel. \\ mogi@bm.technion.ac.il \\ www.bm.technion.ac.il/ mogi
}

Abstract: Low-level consciousness is fundamental to our understanding of the world. Within the conscious field, the constantly changing external visual information is transformed into stable, object-based percepts. Remarkably, holistic objects are perceived while we are cognizant of all of the spatial details comprising the objects and of the relationship between individual elements. This parallel conscious association is unique to the brain. Conscious contributions to motor activity come after our understanding of the world has been established.

Although appreciating the interesting and original thesis presented by Morsella et al., I argue that their depiction of consciousness as merely binding "incompatible skeletal muscle intentions" (sect. 2.4 , para. 5) is extremely narrow. Consciousness is first and foremost a state in which information about the world is made available to us. Information originates from many disparate sources, and it is only within the conscious field that the many inputs create a cohesive, integrative percept. In what follows I present examples showing how consciousness informs us about the world and how complex motor activity is possible while consciousness is engaged elsewhere, and I conclude by using the visual system to discuss the unique nature of consciousness. 
Commentary/Morsella et al.: Homing in on consciousness in the nervous system

Morsella et al.'s description of the "creature in the cave" reaction to the noxious odor takes for granted a highly developed capacity of understanding the world that must precede any meaningful motor reaction. The organism has to understand that its body is an object in a three-dimensional world containing many objects; that its body is an independent object separated from the cave; that there is a world outside the cave; and that the cave has certain spatial properties. It must also be able to estimate distances and sizes that change as it moves. All those consciously gained pieces of information are not necessarily precursors to action and do not represent "competition for control of the skeletal muscles." Calling such consciously gained scene-understanding "encapsulated" is avoiding the issue; the major, fundamental role of consciousness is dismissed to emphasize a minor one.

Driving a car provides a useful example of a complex motor activity that is unrelated to the concomitant conscious field. Occasionally, we may drive while our conscious attention is completely engaged elsewhere; all motor-related decisions, including those involving conflict resolutions (whether to switch lanes now, whether to increase speed), are performed outside our active conscious state. We can find ourselves coming out of a lengthy day-dreaming, realizing that we have been driving $10 \mathrm{ki-}$ lometers without any memory of what happened on the road but with a clear memory of the content of our conscious ruminations.

The nature of conscious perception. When consciously viewing the world, we perceive its many spatial elements together with their associated properties (e.g., location, size, brightness). All those elaborate spatial details are perceived, simultaneously and in parallel. As I show elsewhere (Gur 2015), it is the neural activity in the primary visual cortex (V1) with its veridical depiction of the spatial elements comprising the visual image (a V1 "map") that is the basis of object representation, perception, and recognition. A second process evident in our conscious perception is the integration of the neural activity occurring in disparate V1 loci into holistic objects. The ability of the brain to generate perceived objects is quite remarkable; a small number of dots that individually carry no information of orientation, size, or length can be easily recognized as meaningful shapes (Gur 2015). Even when flashed for a mere 50 microseconds or less (Greene \& Ogden 2012; Greene \& Visani 2015), during which time visual cells fire just one or two spikes (Gur \& Snodderly 1997), it is possible to successfully detect the shapes represented by the dots. Our conscious perception of raw, acute, holistic objects can be modified by processes implemented in many visual and non-visual areas that provide auxiliary information regarding, size, tilt, distance, and so forth. This spread-out activity interacts with the $\mathrm{V} 1$ activity patterns to allow the comparison of the acute, ever-varied consciously observed object with a memory - a stored prototype, leading to object recognition (Gur 2015).

We can thus see that there are two conscious processes that are essential for our visual perception: detail preservation and detailsonto-objects integration, which, together with non-conscious information extraction, enable space perception and object recognition. Needless to say, those conscious processes have nothing to do with motor activity but, among other functions, provide an understanding of our world without which no meaningful motor activity is possible.

It is in the conscious state, particularly the low-level one, that the unique ability of the brain to combine simultaneously occurring discrete local activity patterns into a unified entity is expressed. This instant transformation from the particular to the whole is performed without sending the information elsewhere to a comparator or integrator. Let us consider two points of light that generate two activity loci in V1. These two loci are perceived as two points of light, but, in addition, we can compare their properties: point A is oval, small, and bright, whereas point $\mathrm{B}$ is circular, large, and dim. Such comparisons are done instantly and in parallel. This ability to retain the identity and spatial properties of individual elements and at the same time compare or correlate, in parallel, many individual inputs, is easily performed during conscious perception, but it is something that no physical devise, computers included, can achieve.

Finally, to demonstrate how essential consciousness is to our functioning, let us look at the "creature in the cave" example in some detail: For the organism to react to the noxious odor and move towards the cave's opening, it must understand that there is an opening out there. But the light information falling on its retina is a collection of light intensities of various contrasts and sizes; some stem from the edge of the opening, and some do not. To decide which of the disparate light sources should be combined, and then perceived as a holistic entity that differs from the rest of the cave, is not a trivial achievement. As discussed above, all spatial elements are perceived, some are integrated into the "cave opening object," and some are not. To understand that it is an opening to the outside world requires repeated conscious exposures, interactions, and learning to secure the knowledge that the series of contrast gradients signifies an opening tying the cave to the outside world. All of this and more must happen in the conscious field before the organism can plan any motor activity.

\section{Heavy objects and small children: Developmental data extend the passive frame theory}

\author{
doi:10.1017/S0140525X15002101, e180
}

\section{Cheshire Hardcastle, ${ }^{\mathrm{a}}$ Eliah White, ${ }^{\mathrm{b}}$ Heidi Kloos, ${ }^{\mathrm{c}}$ and Valerie Gray Hardcastle ${ }^{c}$}

${ }^{a}$ Bioscience Division, SRI International and Stanford Hospital \& Clinics, Menlo Park, CA 94025-3493; 'bepartment of Psychological Science, Northern Kentucky University, Highland Heights, KY 41099; ' Department of Psychology, University of Cincinnati, Cincinnati, OH 45220-0376. cheshire.hardcastle@sri.com whitee9@nku.edu heidi.kloos@uc.edu valerie.hardcastle@uc.edu http://artscience.nku.edu/departments/psychology/facstaff/ft-faculty/ White.html

http://www.artsci.uc.edu/departments/psychology/fac_staff.html? eid=kloosa\&thecomp=uceprof

http://www.artsci.uc.edu/departments/psychology/fac_staff.html? eid=hardcave\&thecomp=uceprof

Abstract: Passive frame theory is compatible with modern complexity theory and the idea that conflict drives the emergence of a novel structural organization. After describing new developmental data, we suggest that this conflict needs to be expanded to include not only conflict between action options, but also between action and perception.

Morsella et al. argue that we should understand conscious perception via the interplay between (1) conscious content created through a conflict of action options, and (2) the chosen action after the conflict is resolved. The proposed passive frame theory (PFT) situates consciousness in the context of action-related decision-making, rather than in the context of purposeful symbol manipulation. It is passive in the sense that it is a low-level mediator between different action opportunities. Such action-based consciousness departs from traditional views of consciousness, which typically attribute it to high-level planning and executive function, and moves it down to the level of actions.

There are many strengths in this approach, including its compatibility with embodied cognition and the idea that actionbased processes drive the experience of conscious content (Juarrero 1999; Thompson \& Varela 2001). It is also in line with advances in non-linear dynamics, including the idea that consciousness gives rise to a multi-stable system characterized by self-organized criticality (Bak 1996; Werner 2007). Most importantly, the proposed PFT is compatible with modern complexity 
theory, specifically the idea that conflict drives the emergence of a novel structural organization (Swenson 1997). Here we focus on this latter idea and suggest that conflict needs to be expanded to include not only conflict between action options, but also between action and perception.

There is a large body of research that provides evidence for a discrepancy between actual action capabilities and beliefs about such actions capabilities (e.g., Kozhevnikov \& Hegarty 2001; Krist et al. 1993). The traditional explanation is that such discrepancy is due to two encapsulated systems: an action system, which guides the movements, and a judgment system, which predicts actions. Such dichotomy of structures not only runs counter to PFT, but also is overly simplistic, as illustrated in a preliminary study we carried out to investigate the ability of children to predict how far they can throw balls after feedback.

Participants were 12 children from a Midwestern daycare serving middle-income families. The children ranged in age from 4 to 10 years, half of them being younger than 72 months ( $M=5.04$ years), and half of them being older than 72 months ( $M=7.94$ years). A hallway at the daycare, approximately 16 feet long, was taped off to create an area in which to throw medicine balls. Three medicine balls that differed in heaviness were used. The heaviest medicine ball weighed 6 pounds, the middle ball weighed 4 pounds, and the lightest ball weighed 2 pounds. A yellow square at the end of the hallway acted as a place for the children to stand while throwing the different balls. The researcher prompted each child to "guess how far you can throw the ball." The child was then instructed to stop the researcher, who was walking backwards towards the end of the hallway, in order to indicate how far they thought they would be able to throw the medicine ball. After the child stopped the researcher, the distance between the researcher and the yellow square was measured and recorded. Finally, the researcher left the hallway, and the child was told to "throw the ball." As needed, the researcher reminded the child to throw underhand if the child tried to gain momentum by throwing the ball from the side. The spot where the medicine ball landed was marked, measured, and recorded. This procedure was repeated twice for a total of three trials for each of the balls.

The absolute difference between the predicted distance thrown and the actual distance thrown was determined for each throw and then averaged across trials and children. Figure 1 shows the results obtained. Although the older children were better able than preschoolers to perceive the distance they could throw a ball, there was an interaction between accuracy and ball weight. Specifically, the improvement with age was only for the lightest balls, not the medium and heaviest balls. Their ability to accurately adjust their predictions for how far they could throw the medium and heaviest balls was not statistically different from younger children. Thus, for the heavier balls, older children were no better than younger children at self-correction.

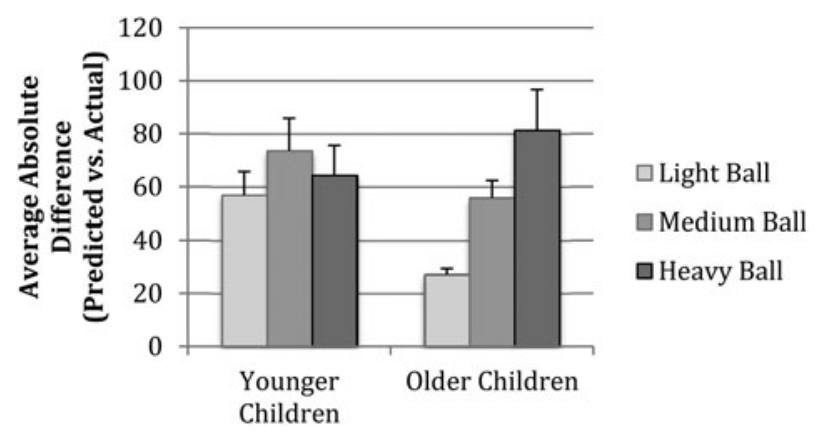

Figure 1 (Hardcastle et al.). Average absolute difference between children's predicted distance thrown and the actual distance thrown, separated by age group and ball weight. Error bars represent standard errors. Measures are in centimeters.
Our results add to the list of findings showing a discrepancy between perceived action capabilities and actual capabilities, even after feedback. Although perception and action can align after experience (e.g., Zhu \& Bingham 2010), and although there can be a positive relationship between preschoolers' selfperceptions of the physical ability and fundamental motor skills (Robinson 2010), this is not always the case (cf. Kloos \& Amazeen 2002). Our results show that such a discrepancy does not fit a theory of independent action and judgment systems. This is because the heaviness of the ball actually matters. To accommodate such context effects, a dichotomous model of action and perception would have to be expanded ad hoc to take into account object weight. Instead, we propose that prediction, as well as action, results from intricate interaction of idiosyncratic constraints, which differ not only as a function of the task ("predict" vs. "throw"), but also as a function of perceptual features (ball heaviness).

The PFT would suggest that older children are better at predicting their "throwability," given that they have had more experience in using conscious information to inform behavioral outputs than preschoolers. Finding that there are circumstances in which predictability does not improve with experience requires expanding PFT. It needs to incorporate not only conflict among potential actions, but also conflict between perception/judgment and action. We conclude that, while compatible with PFT, developmental studies open up the approach to new ways of investigating its tenets.

\section{At what timescale does consciousness operate?}

\section{doi:10.1017/S0140525X15002113, e181}

\section{Bernhard Hommel ${ }^{a}$ and Reinout W. Wiers ${ }^{b}$ \\ ${ }^{a}$ Cognitive Psychology Unit \& Leiden Institute for Brain and Cognition, Leiden University, 2333 AK Leiden, The Netherlands; ${ }^{\mathrm{b}}$ Department of Psychology, Addiction Development and Psychopathology (ADAPT) Lab, University of Amsterdam, 1018 XA Amsterdam, The Netherlands. \\ hommel@fsw.leidenuniv.nlr.wiers@uva.nl www.bernhard-hommel.eu \\ http://www.uva.nl/en/contact/staff/item/r.w.h.j.wiers.html?f=wiers}

Abstract: While applauding Morsella et al. for linking consciousness to action control, we ask what their theory implies regarding the exact functionality of consciousness and the timescale at which it operates. Does consciousness operate on, and resolve the conflict it emerges from (despite its slowness), or does it operate on future conflicts that it might resolve by externalizing/socializing cognitive control?

We applaud Morsella and colleagues for their original, innovative approach and welcome the uncommon, but from an evolutionary perspective very convincing, move (given that evolution operates on actions, not on thoughts) to link consciousness to action control. Many aspects of the theory invite empirical testing, which is likely to stimulate the field. However, we feel that to fully exploit its hypothesis-generating potential, the theory needs to be more specific with respect to the exact functionality that consciousness is assumed to have and to the timescale at which consciousness is thought to operate. We see two possible scenarios, which raise different questions.

The first scenario considers consciousness to operate online, that is, on the same short timescale in which the decisionmaking process operates. This would mean that consciousness not only emerges from/through action-selection conflict, but also operates on that same conflict. How could that work? Conflict solution requires the integration of information (including the conflicting parties and the goal the agent aims at). This fits with the integration consensus but raises the question: In which exact sense do Morsella et al. go beyond the global workspace 
theory of Baars (1988)? It also raises the question of how consciousness could influence the decision-making process involving conflict; is it not too slow to resolve conflict within the $450 \mathrm{msec}$ window that typical responses in conflict tasks take, given that consciousness has been estimated to take hundreds of milliseconds to emerge (Dehaene et al. 2006)? It is this feature, together with the lack of any empirical evidence for an online role of consciousness, that led Hommel (2013) to doubt any online functionality of consciousness. Morsella et al. admit that there are "good reasons" to doubt that consciousness is involved in online action control, but they do not further address this as a problem for their own approach. Moreover, even if consciousness could emerge and operate on time, and even if it would allow for the access to distributed information that speeds up conflict solution, it is not clear why this integration function requires having a conscious experience. Why could a philosophical zombie without any conscious experience not possess such an integrative function? One case where online conscious experience appears to have a strong effect is addiction: conscious craving can overwhelm long-term considerations and lead to actions not consciously intended and later regretted (Kavanagh et al. 2005; Wiers et al. 2014). However, this effect is at a longer timescale than experiments on online action control (seconds to minutes), and its effect is negative in view of the long-term goals of the individual. Hence, a positive example of conscious action control is still wanting.

The second scenario considers consciousness as an off-line function that does not operate during the ongoing perceptionaction event but prepares the agent for later events of the same sort. Rather than resolving the current conflict, consciously representing, reflecting, and communicating about the conflict and/or the solution could contribute to prevent the agent from encountering the conflict again, or at least to prepare her to deal with such conflicts more efficiently in the future. It may be no coincidence that the ability to communicate about an event (i.e., conscious report) is the most widely used technique to assess conscious representations in humans. Rather than merely a methodological convenience, the ability to communicate about conscious states may actually be the essence of what consciousness has evolved to achieve (Baumeister \& Bargh 2014; Baumeister \& Masicampo 2010; Hommel, in press). We may thus represent action conflicts consciously because that allows us to reflect on and communicate the existence of the conflict, our ways to deal with them, and the success of doing so. This allows for social learning and strategy transmission, but also for socializing the conflict.

Several theorists have argued that this indirect social role of consciousness has permitted humans to expand the number of individuals they interact with exponentially (Baumeister \& Masicampo 2010; Levitin 2014). Returning to our addiction example, by telling others about your goal to quit smoking you can mobilize them to help you when dealing with your urge to smoke the next time, this interaction serving as a reminder about your actual goals - you in essence externalize and socialize your goals and executive control functions. In treatment, you may further learn to "surf the urge" (Bowen \& Marlatt 2009) and experience that the conscious urge will also descend when not acted upon. In addition, alternative strategies for weak moments are premeditated: if you often experience strong urges when stressed, it is important to prepare actions other than smoking (e.g., running, meditation, etc.) for upcoming stressful occasions, again externalizing future action control in a desirable way. Given the larger timescale of this operation mode, neither the slowness of conscious representation nor the absence of evidence for online functions of consciousness would be counter-arguments, and it would be obvious why conscious representations need to be conscious. To paraphrase Shariff et al. (2008), although we may subjectively feel that our conscious will operates like a motorboat and we are steering where we want to go, the true operation of consciousness may be more indirect, like a sailing boat, in which we can learn to influence the boat's course in indirect ways, adjusting for the wind and the currents, which will eventually get us to our intended destination.

We would like to invite Morsella and colleagues to become more specific with regard to the timescale of conscious operations and their concrete functionality. This would not only strengthen the approach's potential to stimulate empirical research, but also strongly increase its well-deserved visibility and impact.

\section{Conflicts everywhere! Perceptions, actions, and cognition all entail memory and reflect conflict}

doi:10.1017/S0140525X15002125, e182

\section{Jerome S. Jordan ${ }^{\mathrm{a}}$ and David W. Vinson ${ }^{\mathrm{b}}$ \\ ${ }^{a}$ Department of Psychology, Illinois State University, Normal, IL 61790-4620; ${ }^{\mathrm{b}}$ Cognitive and Information Sciences, University of California, Merced, \\ Merced, CA 95340. \\ jsjorda@ilstu.edu』dvinson@ucmerced.edu \\ https://about.illinoisstate.edu/jsjorda \\ http://davevinson.com/}

Abstract: Morsella et al. assert that the function of consciousness is to determine which of many competing action options is expressed through the skeletomuscular system at any given moment. The present commentary addresses this issue from the first-person perspective and agrees with Morsella and colleagues, yet further proposes that the option-selection function of consciousness plays out in cognition as well.

As I sat to write this comment on Morsella et al.'s action-based synthesis of the science of consciousness, I found myself forced to examine the events that eventually led to my current situation. According to Morsella et al.'s passive frame theory (PFT), the contents that entered into my deliberations would be perceptual representations of external events (e.g., the computer screen in front of me, or the letters that appear on the screen as I type), as well as perceptual representations activated by unconscious corollary discharges, such as my urge to turn my head, look away from the screen, and close my eyes as I try to figure out what to write next. As I turn away, I find myself focusing on PFT's assertion that the contents of perceptual representations are encapsulated. Just then, Proffitt's (2006) economy of action (EoC) theory and Gibson's (1979) theory of direct perception enter my conscious field at roughly the same time. Suddenly, I see myself at a pub, engaged in arguments with dear friends about whether or not perception requires representations. I laugh out loud. Then I realize I have been sitting for some time, my left elbow propped on the desk, my head in my hand, my eyes closed. Eyes open, I realize that I have a co-author on this paper and have yet to include any of his material. I look for the email he sent me, copy a particularly clever section, and paste it into this comment.

My coauthor writes: "While I agree that consciousness influences skeletomotor activity, memories influence reflexive responses within the skeletomotor system as well. This challenges Morsella et al.'s commitment toward conceptualizing cognition using a modular approach. There are many studies, both neural and perceptual, that show how action-based memories are integrated into efference streams affecting planned skeletomotor action. Specifically, motor-cortical regions associated with action planning are more active when viewing dancers whose expertise is similar to one's own (Calvo-Merino et al. 2005), suggesting that the observation of another's actions activates past action plans associated with moving oneself. If so, one's perceptions should be influenced by the observation of another's actions when they elicit motor-cortical activation associated with acting. Perceived distance estimates increase when observing another carry a weighted backpack only if the observer previously carried the weighted backpack and is 
Commentary/Morsella et al.: Homing in on consciousness in the nervous system

now not carrying the backpack. That is, only after the observer has developed backpack-carrying memories, are distance estimates affected by the observation of another's actions (Vinson et al. 2015). Findings such as these suggest that memories are driven into the very makeup of efference streams within one's skeletomotor system (possibly stored in cerebellar cortical networks; see Jordan 2013). Thus, cognition cannot be modular when past actions are nested within the very nature of the contents that constitute perception."

Seeing my name, particularly when paired with “2013," reminds me to mention that the "memories" my co-author is referring to are due to the fact that neurons in the motor cortex project collaterals to very specific locations in the cerebellum, all of which recursively innervate the cortical neurons from which they receive input. These very same cerebellar neurons receive input from the afferent systems influenced by the movements resulting from motor neuron stimulation. As a result, neurons in the cerebellum allow one to embody "command-feedback" regularities, or, if you like, successful movement-effect memories. As a result, the neural activity in the cerebellum is neither wholly "motor" nor "sensory." It is simultaneously both, and neither. Recognizing the neither "sensory" nor "motor" nature of the "memories" entailed in cortico-cerebellar circuits is important because these cerebellar neurons recursively influence motor cortex. As a result, the content of the neural activity in the motor cortex is rendered neither "motor" nor "sensory." It, too, is simultaneously both. This dissolving of the sensory-motor divide strongly challenges the "motor-sensory," "perception-action," "input-output" distinctions that lie at the heart of PFT.

I read over the preceding paragraph, just to make sure I've not generated a plateful of Escher spaghetti. No - it works, and it only potentially sounds bizarre because of our historical, theoretical commitment to conceptualizing perception as input and action as output. Then I remember to mention the fairly recent discovery that almost every area of the cortex shares recursive coupling with the cerebellum, just as is the case with the motor cortex (Koziol et al. 2011; Schmahmann 2001). This means activity in most, if not all, cortical areas is continuously, recursively influenced by the cerebellum. In short, activity in the cortex is continuously primed by the recursive "memories" stored in cortico-cerebellar circuitry.

I scratch my back, trying to remember why I started writing about the cerebellum. I look at the screen and remember. If the contents of the conscious field are there to help determine which pattern of skeletomuscular behavior is expressed at any given moment, then the bizarre, seemingly distorted pattern of thoughts described above should not have been conscious. But they were. As I read back over them, I realize that almost every moment of change in content could very well have reflected a moment of planning conflict-not about what my body would do at that moment, but what my "thoughts" would "do" at that moment. Thinking about encapsulation led to my thinking about Proffitt (2006) and Gibson (1979). Thinking about Proffitt and Gibson led to my thinking about representations and friends in a pub. In each case, the content of the thoughts seemed to reflect conflicts emerging from previous thoughts. Apparently, Morsella and colleagues are correct: Consciousness is about conflicts - but not just skeletomotor conflicts. Rather, as a result of the fact that cortical activity is continuously primed by the memory content entailed in cortico-cerebellar circuitry, the cortical activity associated with perception, and action, and cognition is always potentially in conflict. That is, attempting to maintain a given thought, perception, or action will result in conflicts as both exogenous contextual factors, and endogenous memories will prime actions, perceptions, and cognitions that are inconsistent with what I am attempting to do. In short, as I work to make any one thing happen in perception, action, or cognition, it will inevitably be in conflict with the myriad other things I could have possible done, and "consciousness" will entail this ebb and flow of conflicts.
As I rest my head on the desk and close my eyes, I feel a sense of satisfaction. I suddenly remember my co-author and the need to send him the final draft. Will the conflicts never cease?

\section{Is conscious content available only to the skeletal muscle system?}

\section{doi:10.1017/S0140525X15002137, e183}

\section{Andreas Keller \\ Laboratory of Neurogenetics and Behavior, The Rockefeller University, New York, NY 10065. \\ andreasbkeller@gmail.com}

Abstract: I applaud Morsella et al.'s approach to investigate consciousness in terms of behavioral control. After all, the function of the brain is to control behavior, and consciousness contributes to the function of the brain. However, I question whether conscious content is available only to the skeletal muscle system, as the principle of parallel responses into skeletal muscle (PRISM) (Morsella 2005) proposes.

Morsella et al. write that conscious content is "sampled" only by the action systems in charge of controlling skeletal muscles (sect. 4.3, para. 3). They state that this has been known since at least the nineteenth century, but they do not discuss the evidence on which this knowledge is based. Here, I will discuss examples of conscious content being available to bodily effectors outside of the skeletal muscle system and suggest that the function of conscious content is not to serve a specific effector system, but rather to control adaptive behaviors in general.

One example of an output system other than the skeletal muscle system that has access to conscious content is: the glands responsible for salivary secretion. These glands are controlled exclusively by the autonomic nervous system (Proctor \& Carpenter 2007). Although not controlled by skeletal muscles, salivary flow is responsive to conscious content. Simply forming a mental image of one's favorite dessert induces salivation. This widely shared experience, which also has been confirmed experimentally, shows that salivary glands have access to the content of consciousness (for a review, see Spence 2011).

Just like fantasies about food can make the mouth water, erotic fantasies can result in sexual responses that are independent of the skeletal muscle system, for example, penile erections in men. Both in the case of imagery-induced salivation (White 1978) and in the case of penile erection (Smith \& Over 1987), subjects with more vivid imagery showed more pronounced effects, indicating that there is a close correlation between the strength of the conscious content and the strength of the bodily response outside of the skeletal muscle system. This correlation does not prove causation. The mental images of food and the increased salivation could both be caused by the same non-conscious neuronal processes and therefore be independent consequences of the same cause. However, this argument for the potential causal inertness of conscious content applies equally to cases in which the skeletal muscle system is the effector as to those in which there are other effectors.

According to Morsella and colleagues, when a food object is represented, the conscious content does not "know" whether the food will be eaten or used as a weapon. This is true, but it does not go far enough. The conscious content also does not "know" whether the representation of the food item will induce any muscle movement, or whether it will induce salivation, or both. When the brain integrates information, it does not differentiate between information that will ultimately be used to control skeletal muscles and information that will be used for other purposes. The conscious content is made available to any system to which it is of adaptive advantage to have it.

To illustrate the analogy between situations in which action selection conflicts concern skeletal muscles and situations in which action selection conflicts concern salivary glands, one can 
modify the authors' "creature in the cave" scenario so that it applies to both situations. In the "creature in the cave" scenario, an organism has to decide whether or not to leave a cave that is comfortably warm but slowly fills with (uncomfortable) smoke. A similar conflict can occur with salivation. Food stimuli or thinking about food increases salivation, whereas the thought of anxiety-inducing situations reduces salivary flow (Power \& Thompson 1970). A creature can therefore be in a situation in which these two tendencies are in conflict. Imagine a scavenger observing a predator feeding on its prey. The scavenger has to make a decision whether to approach the predator and its prey and expose itself to danger while feeding, or whether to stay hungry but safe. This is an action selection conflict in which the actions are mediated by the skeletal muscle system. However, the same action selection conflict is faced in this situation by the salivary glands, which can either increase or reduce salivary flow. The conflict is experienced consciously, and the conscious content is made available to both the skeletal muscles and the salivary glands.

In summary, the evolutionary function of conscious brain processes, like the evolutionary function of all brain processes, is to contribute to the selection of adaptive behaviors. However, the evidence that consciousness is a special adaptation to the processing of information that is exclusively used by the skeletomotor system is not conclusive. An alternative proposal is that consciousness is important to guide behaviors in situations in which the organism has to choose between many possible responses, regardless of the effector system concerned (Keller 2014). The conceptual framework that Morsella et al. have developed will be a helpful tool for identifying the role of consciousness in the control of adaptive behavior.

\section{The chemosensory brain requires a distributed cellular mechanism to harness information and resolve conflicts - is consciousness the forum?}

doi:10.1017/S0140525X15002149, e184

\section{Richard Lathe $e^{\mathrm{a}, \mathrm{b}, \mathrm{c}}$ \\ ${ }^{a}$ Division of Infection and Pathway Medicine, University of Edinburgh, Little France, Edinburgh EH16 4SB, United Kingdom; ${ }^{\mathrm{b}}$ Pieta Research, Edinburgh EH10 5YW, United Kingdom; ' ${ }^{\circ}$ State University of Pushchino, 142290 \\ Pushchino, Russia. \\ richardlathe@ed.ac.uk}

\begin{abstract}
The central nervous system (CNS) evolved from a chemosensory epithelium, but a simple epithelium has limited means to resolve conflicts between early drives (e.g., approach vs. avoid). Understanding the role of "consciousness" as a resolution device, with specific focus on chemosensation and the olfactory system, is of appeal. I argue that consciousness is not the adjudicator, but is instead the forum that brings conflicting (conscious) inputs into a form that allows them to be (unconsciously) compared/contrasted, guiding rational action.
\end{abstract}

It was previously argued (Merrick et al. 2014; Shepherd 2007) that olfaction is central to understanding consciousness. In the target article, Morsella et al. extend this framework and argue that consciousness "is for adaptive 'voluntary' action" (sect. 2.4, para. 1), including "action selection" and the integration of conflicting inputs. In addition, they assert that "olfaction provides the best portal for understanding the neural correlates of additions to the conscious field" (sect. 3.5, para. 9). Their account is very welcome; I suggest possible additions to their narrative that might strengthen their case.

That chemosensation, notably olfaction, is central to understanding brain function recognizes that the vertebrate brain has its evolutionary origins in a simple chemosensory epithelium (Nieuwenhuys et al. 1997). To this, one must add that a divergence occurred early in vertebrate evolution, with the olfactory/taste/vomeronasal systems becoming responsible for sensing external chemical stimuli (exteroception), whereas the limbic system (a derivative of the olfactory system) senses the internal milieu (Lathe 2001; Riss et al. 1969). This view (limbic enteroception) is supported by evidence that acquired responses can be guided by internal body states, which are abolished by hippocampal lesions, and by molecular evidence that the hippocampus is selectively adorned with receptors for hormones and metabolites (Lathe 2001). Therefore, if olfaction plays a central role in consciousness, so too must the limbic system (e.g., Behrendt 2013).

Regarding consciousness, we are undoubtedly "aware" of both external stimuli (tastes, smells) and internal stimuli (e.g., blood glucose and oxygen levels; see subsequent discussion). It should be noted that goal-oriented cravings of the hunger type are not restricted to calories (low blood glucose), and specific hungers can be driven by deficiencies in vitamins and specific minerals (Denton 1982). Other hippocampal inputs monitor other body states relevant to immunity and reproduction. However, exactly as for odors, perception of the millions of molecules to which we are constantly exposed both internally (and externally) remains subliminal; we only become conscious of internal states (e.g., sickness, stress, hunger, etc.) when these (or their lack) surpass a threshold. In addition, we are surely aware of other factors, such as the time of day, ${ }^{1}$ possibly also via the hippocampus (Lathe 2001).

Hence, to the central model of Morsella et al.: A hypothetical organism resides in a warm cave, and perceives an opening from which it could exit. The authors expand: "but then it perceives a noxious smell (e.g., smoke) from within the enclosure. Because of this new conscious content, it now exits hesitantly through the opening, even though it was inclined to remain within the warm enclosure" (sect. 1.3, para. 2). Later in their text, this scenario is revisited, and Morsella et al. observe that "the conscious content about the smell triggers a conscious content representing an avoidance tendency toward the smell" (sect. 5, para. 7)

The reader will perceive the following problem: Smoke is not necessarily aversive (smoked salmon is a delicacy, and many deem the odor of aromatic pipe tobacco to be attractive). The key word is "noxious." Why does this creature avoid the smoke? How does the creature "know" that smoke is toxic? Two different scenarios present themselves.

1. The chemoreceptors for smoke components in this creature have been selected, over millions of years of evolution, to be wired up as innately aversive - in the same way that we respond reflexively with disgust and aversion to highly toxic $\mathrm{H}_{2} \mathrm{~S}$.

Or,

2. The creature, remaining in the cave (i) perceives both the smoke (exteroception) and the adverse effects of exposure to molecules accompanying the smoke, notably $\mathrm{CO}$ and $\mathrm{CO}_{2}$, but also volatile organic compounds. (ii) The creature then becomes conscious of feeling sick and dizzy as a result of anoxia and toxemia (enteroception). Then, (iii) the creature makes an (unconscious) inference that the new smell is associated with the new sickness. Finally, (iv) it takes the decision to leave the cave.

If we were asked which of these processes best equates to the function of consciousness, we would probably say "decisionmaking based on comparing memory against sensation." Or, in the terms of Morsella et al., action selection from multiple inclinations.

However, consciousness is distinct from both decision-making and memory. There is evidence that decisions are taken many seconds before they become available to consciousness (e.g., Soon et al. 2008). Furthermore, consciousness is not memory either; we are only aware of a tiny subset of memory traces at a time. In other words, the consciousness circuits must be different from the sensory and memory circuits.

In addition, an important component of consciousness is "what one is thinking about." This is clearly guided by internal and 
external sensory inputs. For example, if I am hungry, I think about food - where it might be found, how to procure it (even while I am engaged in another activity such as walking to work). But if I trip and fall, in subsequent actions pain takes precedence over hunger. Guided selection of what we are thinking about is central to consciousness

I venture that consciousness might best be thought of as the forum in which relevant current and former experiences and associations are borne in mind and cogitated upon, but it does not itself take the decision.

I would liken the overall process of decision-making to a trial, where the relevant evidence is assembled and deliberated upon in a single "place" (the forum or court-consciousness), including access to the different accounts and previous relevant decisions from the legal archive (memory), such that the judge and jury, interacting in camera (unconscious), can hand down a ruling to the forum (conscious mind) - that dictates action at the same time as it becomes conscious. This would require several different circuitries operating in parallel.

In short, I feel that the joint focus on consciousness, chemosensation, and conflict resolution that Morsella et al. propose is an excellent step in the right direction. However, I add the caveat that the circuitries/mechanisms may be subdivided into several subsets that fulfill different functions-conscious assembly (Forum), memory (Archive), and unconscious decision-making (Judge and Jury) - and that consciousness alone does not resolve conflicts.

\section{NOTE}

1. We are also conscious of time of day, and that this can dictate when an action is appropriate or inappropriate. One is reminded of Gallistel's bees, which, encountering a garden breakfast table one morning, return exactly 24 hours later in the expectation that marmalade will again be on the table (Gallistel 1993).

\section{Consciousness for perception and for action: A perspective from unconscious binding}

\author{
doi:10.1017/S0140525X15002150, e185
}

\section{Zhicheng Lin \\ Department of Psychology, The Ohio State University, Columbus, $\mathrm{OH} 43210$. zhichenglin@gmail.com \\ https://sites.google.com/site/zhichenglin/}

Abstract: I argue that the scope and strength of unconscious perception have been overestimated in extant theories. I describe an unconscious binding perspective, and how in conjunction with rigorous methodology it can guide the delineation of unconscious processing. Under this perspective, the function of consciousness is to increase the saliency of conscious contents by facilitating the deployment of focal attention.

Theories on the function of consciousness abound, with the latest from Morsella et al. (target article's passive frame theory) describing that consciousness is for adaptive voluntary action. This actionbased function complements previous functions - such as social interaction and language-assigned to consciousness. While these theories share little in common, they all appear to be at odds with a simple observation: that perceptual understanding of a scene involving complex binding of the constituent parts has never been demonstrated to be free from consciousness. Consider the painting The Starry Night. While the conscious mind can easily appreciate its visual meaning - a village in the moonlight there exists no evidence that the unconscious mind is capable of scene understanding of this sort.

This leads me to argue, in the following section, that unconscious perception may be more limited than is thought in extant theories (including the action-based theory). As a remedy, I then describe a conceptual framework that integrates perception and action based on the notion of binding, and explain how such a binding perspective, combined with rigorous methodology, can guide us to delineate the scope and limits of unconscious processing. The function of consciousness appears to be to increase the saliency of conscious contents by facilitating the deployment of focal attention - in the service of both perception and action.

The scope and limits of unconscious perception, and what it means to the function of consciousness. Integral to the actionbased theory is the notion that complex perceptual integrations, such as feature binding and intersensory binding, can take place without consciousness. However, this premise regarding the power of unconsciousness rests on a sand-like ground. Although it is generally accepted that some perceptual processes can occur without consciousness, it is far from clear that binding processes such as feature binding and intersensory binding can do so as well. This is largely because of two related reasons: demonstrating a process as being unconscious requires rigorous standards; and developing optimal task conditions to reveal the power of unconsciousness is challenging.

The action-based theory considers an event unconscious when subjective experience regarding the event is lacking. Relying on subjective experience to establish unconsciousness, however, conflates consciousness with the idiosyncratic criterion for consciousness. One may, for example, report a stimulus as invisible when one can partially see it, owing to a shy, conservative personality (Bjorkman et al. 1993). Even objective methods, which determine the level of consciousness by measuring the accuracy in forced choices, must be carefully vetted. Indeed, underestimation of consciousness has been demonstrated in recent studies reporting a phenomenon called priming of awareness (Lin \& Murray 2014a; 2015a), which explains the underestimation of consciousness in a collection of studies on unconscious cognitive control. Therefore, it is important to distinguish just what criterion we use - subjective versus objective-when we talk about unconscious processing, and whether the method used allows us to infer unconscious processing.

Mindful of the rigorous standard required to establish unconsciousness, the task of developing optimal conditions to reveal the power of unconscious processing becomes a challenge. Although there is some evidence for unconscious perceptual binding-such as the processing of object relations (whether two objects are of the same or different shapes; Lin \& Murray $2014 \mathrm{~b}$ ) and object congruency with its context (playing with a ball vs. a watermelon in a basketball court; Mudrik et al. 2011) - how robust and reliable these effects are remains to be tested. By all accounts, these unconscious binding effects are rather small compared with conscious effects. It is not that unconscious effects are necessarily weaker than their conscious counterparts; the reverse case has been demonstrated recently in the context of attentional cueing (Lin \& Murray 2015b). This raises the question of why unconscious binding appears to be much more limited and weaker than conscious binding.

One might lay blame on weaker sensory stimulation and less attention being allocated in the unconscious condition than in the conscious condition. Equating sensory and attentional strengths between conditions, however, may turn out to also equate our phenomenological experience in these conditions, abolishing the distinction between unconsciousness and consciousness. The very function of consciousness, then, may well be to allow salient information to bob up into the conscious field, facilitating the deployment of focal attention to furnish further operations such as sophisticated binding, thinking, and reasoning.

An unconscious binding perspective of perception and action. It is clear from the preceding discussion that we lack relevant data to evaluate the scope and limits of unconscious processing. How can we push our understanding forward? Here, the notion of binding in perception research can be naturally extended to the domain of action, because adaptive action, too, requires a solution to the binding problem. Lifting a teacup by its handle, for example, requires knowledge of spatial relations between 
features (the body and the handle) and the object to which they belong (the teacup). Binding, therefore, is required for both perception and action.

In the domain of perception, it has been proposed that certain types of visual binding can occur without consciousness, and such unconscious binding is more fragile than conscious binding ( $\operatorname{Lin} \&$ He 2009). Given the central role of binding in perception, such an unconscious binding framework, by leveraging rich psychophysical, physiological, and computational understandings in the binding problem, could be useful for guiding us to probe the scope and limits of unconscious processing - as well as how unconscious processing interacts (binds) with conscious processing (Lin $\&$ Murray 2013). For example, certain binding processes may be more critical than others in enabling perceptual consciousness (e.g., perceiving the teacup), and they might not be the same as those critical for enabling action consciousness (e.g., lifting the teacup). The central question then becomes just what kinds of binding require consciousness and what kinds of binding do not, and to what degree - in both perception and action. Such an unconscious binding perspective provides an informative direction along which one can delineate the scope and limits of unconscious processing, the knowledge of which is indispensable if we are to understand the nature of consciousness.

\section{Locating consciousness: We are conflicted by the role of conflict}

\section{doi:10.1017/S0140525X15002162, e186}

\section{Dom Massaro and Bill Rowe}

Department of Psychology, University of California, Santa Cruz, Santa Cruz, CA 95060.

massaro@ucsc.edu wrowe@ucsc.edu

http://mambo.ucsc.edu/dominic-massaro/

Abstract: What is consciousness for? Consciousness allows us to handle conflict, a promising proposal by Morsella et al. However, they provide little evidence as to why consciousness is particularly valuable in resolving conflict, nor do they limit the role of consciousness to only conflicting experiences. We attempt to clarify their possible positions and offer several solutions for how these might be formulated and tested.

Bill is expounding on the genius of Julian Jaynes (2006) and how his theory of consciousness also meshes with the view of Nicholas Humphrey (2006). I want to interject a questioning voice, but I was just about to enjoy a unique blend of hops by lifting my fresh glass of our local Santa Cruz IPA. This situation fits with Morsella et al.'s conflict-do I utter my question or bring the glass to my lips to savor the beer? The situation is not as dramatic as a driver seeing a deer jump across a mountainous road, creating a conflict of whether to continue on and risk hitting the deer or to swerve off the road. Consciousness manages these scenarios of conflict, according to Morsella et al., but they do not justify why consciousness makes the resulting behavior any easier or more adaptive.

More importantly, perhaps, the authors appear to assume that consciousness is present well before the conflict begins. Their cave example has the actor consciously perceiving the cave's opening and having the conscious experience of a noxious smell. Thus, our actor is conscious of the relevant percepts without any conflict, so consciousness cannot be limited to solving only conflict situations. Therefore, the question for Morsella et al. is whether the person is conscious of both stimuli before any conflict arises, or whether the conflict is responsible for a conscious experience of these two stimuli. If Morsella et al. really impute consciousness of fundamental percepts like seeing and smell, then they have joined the dominant camp of attributing consciousness to our direct experience of objects and events.

A more unique position would be to define the individual percepts as non-conscious, the outcome of prototypical pattern recognition processes that could easily be performed by a zombie or automaton without consciousness. Consciousness would raise its talking head only when the individual percepts create conflict as in our examples of taste versus talk or our actor leaving or staying in the cave.

But we think that Morsella et al. won't deny that percepts are conscious. To resolve this conflict, we might turn to Seeing Red by Nicholas Humphrey (2006). He attributes consciousness not to percepts but to sensations created by the interaction of the senses and an experiencing actor. So for Humphrey, we have a conscious actor interacting with various percepts unconsciously until the deer jumps across the road. Would this second engagement of Humphrey's consciousness perhaps now access something from the sensorium to better deal with the conflict? If so, benefitting from the sensorium's input would seem to justify consciousness during conflict but not during individual (non-conflicting) percepts. However, we doubt it would access anything beyond the normal ongoing activity of the sensorium. It might access something from the "narratorium." That is, along with the provoked emotional shift of seeing a deer, the individual might streamline any number of narrative scenarios about what is going on and what to do about it.

In Morsella et al.'s model, it is almost certain that having a deer jumping across the road could cause a consciousness-evoking change. The driver may have been in one of those episodes that people talk about where you drive for miles with no recall of being aware of that driving. Then the deer appears. Now, for Morsella et al., one conflict might be the following: "Do I do less damage to myself and the car by swerving or by continuing on?" In Morsella et al.'s view, these competing actions that are vying for access to the motor output system bring about a moment of consciousness.

How many conflicts do we actually run into? Is conflict all that unique, infrequent, or qualitatively different? Our mindless pattern recognition solves conflicts seamlessly, as in our studies of optimal speech perception, given auditory and visible speech (erroneously called the McGurk effect because it involves much more than the field's prejudice for "illusions" given conflicting speech inputs). Conflict does not seem to unravel this form of integration as specified by the Fuzzy Logical Model of Perception (FLMP; Massaro 1998).

In an expanded 5-by-5 factorial design, for example, five levels of audible speech varying between $/ \mathrm{ba} /$ and $/ \mathrm{da} /$ were crossed with five levels of visible speech varying between the same alternatives. There were also trials that had simply a visual or an auditory syllable. The participants were instructed to identify the syllable as /ba/ or /da/. The results showed a large interaction between auditory and visual speech, a signature prediction of the FLMP. There appeared to be a natural integration of auditory and visual speech even though many of the speech events had conflicting audible and visible speech. This result might lead us to question why conflict is necessary or sufficient for consciousness.

Perhaps one could empirically test whether behaving to a single percept is all that different from behaving to conflicting percepts. This would inform whether conflict is all that is essential to engaging or creating consciousness. Recall the demonstrations of Benjamin Libet in which an actor was told to watch a clock and decide when to make an arm movement (see Obhi \& Haggard 2004). She moved and then reported what time the clock was showing when she decided to move. The results revealed that an action potential in the brain occurred before the time she reported. We could replicate this experiment and also include a conflicting situation in which there is a conflict and the person judges when he or she decided to make one action versus another, such as move one hand versus another. For example, a right hand results in five people dying and a cure for the common cold, whereas a left hand response results in 10 people dying and a cure for cancer. The question would be whether the action potential in the brain occurs before or after the time the person reported making a decision. If the conflict situation reverses the classic 
result, which would mean that the action potential occurred after the time the person reported making a decision, then we might have empirical evidence for something like consciousness influencing conflicting situations relative to non-conflicting situations.

\section{Origins of emotional consciousness}

\author{
doi:10.1017/S0140525X15002174, e187
}

\section{Hans L. Melo a, Timothy R. Koscik ${ }^{a}$, Thalia H. Vrantsidis ${ }^{a}$, Georgia Hathaway ${ }^{a}$, and William A. Cunningham ${ }^{a, b}$ \\ ${ }^{a}$ Psychology Department, University of Toronto, Toronto, Ontario, M5S 3G3, Canada; ${ }^{\mathrm{b}}$ Rotman School of Management, University of Toronto, Toronto, Ontario, M5S 3G3, Canada. \\ hans.melo@mail.utoronto.cart.koscik@utoronto.ca \\ thalia.vrantsidis@mail.utoronto.ca georgia.hathaway@mail.utoronto.ca cunningham @psych.utoronto.ca \\ http://socialneuro.psych.utoronto.ca/}

\begin{abstract}
While the field of emotions research has benefited from new developments in neuroscience, many theoretical questions remain unsolved. We propose that integrating our iterative reprocessing (IR) framework with the passive frame theory (PFT) may help unify competing theoretical perspectives of emotion. Specifically, we propose that PFT and the IR framework offer a point of origin for emotional experience.
\end{abstract}

The nature of emotional experience continues to captivate scientists and human society in general. With modern neuroscientific methods, there is renewed progress in uncovering mechanisms underlying emotional experience. That said, the field remains divided by fundamental, theoretical disputes and a lack of consensus on the definition of emotion or what constitutes emotional experience. We propose that integrating our iterative reprocessing (IR) framework with the passive frame theory (PFT) of consciousness may help unify competing theoretical perspectives of emotional experience. Because emotions can be conceptualized as multimodal synchronizations of component processes that direct action, we suggest here that a theory of consciousness in the service of action is informative for the understanding of emotional experience.

Basic emotion theorists propose that a limited set of basic emotions evolved as adaptive, whole body responses to environmental challenges (Ekman 1992). In contrast, psychological constructivists propose that emotions arise from the interaction of basic processes, such as interpretations of ongoing mental activity, or core affect, organized through semantic conceptualization (Barrett 2006; Russell 2003). Specifically, psychological constructivists see emotions as conceptual interpretations of core affect, defined by valence (positive or negative) and arousal (intensity), where differentiated emotions arise when states are categorized. Similarly, appraisal theorists propose that emotions arise from specific cognitive interpretations, such as whether an event is relevant to current concerns (see Ellsworth \& Scherer 2003). Critically, for some (e.g., Frijda et al. 1989), action tendencies associated with a cognitive interpretation fundamentally constitute the emotional experience. Although these later perspectives provide formal cognitive models of emotion, they are often silent with regard to how and when these processes of interpretation arise in our subjective experience.

Our view of emotion is part of a growing consensus that the distinction between emotion and cognition is a false dichotomy (Cunningham \& Kirkland 2012); if we define cognition as information processing, it follows that emotion is a type of cognition. The IR framework exploits this perspective to understand emotion as the emergent result of hierarchically organized, dynamic, neural processes (Cunningham et al. 2013). Central to this framework, information is processed through iterative cycles as individuals interact with their environment, and each iteration provides richer evaluations and more nuanced interpretation of information being acquired. Critically, the goal of the mind is to settle into a stable, predictive, internal representation of the environment, similar to a system going from a high to low entropy state. We propose that when percepts with conflicting action tendencies arise in this iterative cycle, the entropy or degree of randomness increases (Clark 2013; Friston 2010), triggering what people label as emotion. This coincides with Morsella et al.'s proposal that conflicting representations can re-iterate through the conscious field to guide coherent action.

Importantly, the brain attempts to reduce overall entropy in its information processing in order to maintain stability. One way to maintain stability is by selecting and executing actions that resolve ongoing conflict, or emotion, which relaxes the system into a stable, lower entropy configuration. This process of conflict resolution occurs dynamically and continuously as new percepts perturb the system (Spivey 2008). This perspective links with Morsella et al.'s PFT; emotional consciousness results from the dynamics of conflicting options for action. In this sense, the only difference between consciousness and emotional consciousness is the conceptualization of the current conscious state as being emotional. We believe that PFT has implications for our understanding of emotional consciousness, whereby what we often label emotional experience emerges, at least in part, from conflicting representations that often direct voluntary action.

This perspective incorporates the role of cognitive interpretations emphasized in constructivist and appraisal models, in that conscious emotional experience is an interpretation of unconscious mental states. This view can also incorporate the role of valence and arousal used in constructive models of emotion. Rather than assuming that these are basic dimensions of core affect that get interpreted, we instead propose that components of the conflict resolution process can emerge into consciousness and be interpreted as valence and arousal. In addition, this view highlights the importance of action tendencies for emotional experience; emotions arise into consciousness in response to conflicting action tendencies, and, as conscious contents, can further guide voluntary action.

This formalization integrates with our IR framework, which emphasizes the dynamic unfolding of affective-cognitive processes. Within this framework, information is able to flow both forward and backward within hierarchical systems, where previous iterations serve as inputs to the overall process or conscious field. We propose that the origins of emotional experience lie directly within consciousness as outlined by Morsella and colleagues. We believe that PFT and the IR framework offer emotion theory a point of origin from which we might form an integrative theory of emotional experience.

\section{Conscious olfaction: Content, function, and localization}

\author{
doi:10.1017/S0140525X15002186, e188
}

\section{Bjorn Merker}

Fjälkestadsv, 410-82, SE-29194, Kristianstad, Sweden.

gyr694c@tninet.se

Abstract: The target article's emphasis on olfaction is a welcome reminder of the multimodal nature of conscious experience. Here, I explore the distinctive and even unique attributes of our sense of smell from the point of view of their bearing on and fit with a subcortical locus of sensory experience.

In outlining a conceptual framework bearing on the organization and function of consciousness, Morsella et al. highlight the olfactory system as a test-bed and challenge for consciousness theory.

A trenchant comparison between olfaction and other senses has been provided by Köster (2002). For present purposes, olfaction stands out among our exteroceptive modalities in four principal respects: 
Commentary/Morsella et al.: Homing in on consciousness in the nervous system

1. Its minimal "spatialization," extending - in humans - to no more than a rudimentary left-right directionality, if any (Boireau et al. 2000; Frasnelli et al. 2009; Kobal et al. 1989; Porter et al. 2005).

2. The ready, indeed profound, habituability of "ordinary odorants" (as noted by the authors and discussed in more detail below).

3 . Its heavy investment with innate odorant aversions and preferences (Khan et al. 2007; Kobayakawa et al. 2007; Mandairon et al. 2009; Mori \& Sakano 2011; Nishizumi \& Sakano 2015; Yeshurun \& Sobel 2010).

4. The extent to which some of its functions complete themselves without being represented in awareness, as in pheromonal signaling (Albrecht et al. 2011; Hummer \& McClintock 2009; Lundström \& Olsson 2005; Lundström et al. 2008; MujicaParodi et al. 2009; Saxton et al. 2008; Wyart et al. 2007; Zhou \& Chen 2009; see also McClintock 2000; Wysocki \& Preti 2004).

Olfactory information that does enter or intrude on awareness does so in much the same way as information carried by other conscious modalities - namely, to lay claim on the deployment of our musculoskeletal resources for some instrumental purpose or other (Merker 2007, p. 73; Morsella 2005). Thus, we arrive at the target article's "creature in the cave" example, or the jolt which, at the first faint whiff of burnt broccoli that reached my nostrils while engrossed in writing this commentary, sent me dashing to the kitchen to save my lunch from being turned to charcoal. The diamines cadaverine and putrescine on the one hand, and fruity esters on the other, exemplify odorants that make us steer our steps away from or towards natural sources of toxins or nutrients by filling our consciousness with unlearned powerful revulsion or more subtle attraction, respectively. Many an ordinary odorant does the same on the basis of learned associations, or acts simply as a novel or unexpected stimulus, eliciting the musculoskeletal orienting reflex (Sokolov 1963) in search of its source.

In the absence of direct demands on the muskuloskeletal system, as in odorant effects on general propensities evidenced in mood, attitudes, or hormonal status, the operations of the olfactory system often proceed without awareness of the odorant itself (see references in the numbered list, point 4 above). The olfactory system thus offers natural opportunities for "contrastive analysis" (Baars 1997a) by tracing differential information flow in cases of subliminal versus conscious olfaction. This is all the more so because the olfactory bulb in humans, who apparently lack a functional vomeronasal organ (Mast \& Samuelsen 2009; Savic et al. 2009), carries both pheromonal and ordinary odor information to the brain (Mori \& Sakano 2011; see also Friedrich 2011).

In attentional terms, conscious olfaction behaves like other sensory modalities at least to the extent that attention to olfaction facilitates odor detection. This facilitation takes place in the presence of increased functional connectivity along the "indirect pathway" from piriform to orbitofrontal cortex via the mediodorsal nucleus of the thalamus (Plailly et al. 2008). The lack of olfactory "spatialization" makes attention of the directional kind largely irrelevant to olfaction (Sela \& Sobel 2010).

There is, however, a particular attentional effect that could in principle be shared with the other senses but appears to be altogether lacking in olfaction. In other exteroceptive modalities, information that has disappeared from awareness on account of habituation (say, the humming of a refrigerator, or the touch of one's clothing on the body) can be restored to awareness by an act of deliberate attention. Not so for olfaction (Köster 1971; 2002; Stevenson 2009). There is simply no way to restore the habituated smell of one's home to awareness by attentional effort. Typically, its characteristic smell is detectable only after returning home from a prolonged absence. This is the profound habituability of ordinary odors (as opposed to some "extraordinary" ones: try habituating to the smell of cadaverine!) mentioned in point 2 above.

This unavailability of ordinary odorants to deliberate dishabituation is a direct consequence, I suggest, of the fact that the "deliberately dishabituable" modalities enter the telencephalon via a first-order thalamic relay. Olfaction, on the other hand, enters directly, by making its first central synapses in allocortical prefrontal and immediately adjoining medial temporal areas without first having passed through the thalamus (Carmichael et al. 1994). This places olfaction outside the umbrella of the mechanism by which cortical layer VI pyramidal cells "tune" information on its "way up" to the cortex from first-order nuclei (for which see Merker $2013 \mathrm{c}$, p. 2), an arrangement that may allow deliberate dishabituation of the affected modalities to take place. To the best of my knowledge, this is the first time this anatomical arrangement is proposed to bear on dishabituability and its lack in olfaction, a conjecture which this commentary allows me to commit to print.

How, then, does olfactory information become conscious, when it does? That, of course, depends upon what your "paradigm of consciousness" dictates regarding the necessary and sufficient conditions for information to attain conscious status. In a series of previous publications, I have elaborated one such paradigm (Merker 2007, 2012; 2013a; 2013c). It proposes that cortical operations, cast in probabilistic form, would benefit from having final estimates precipitated extra-cortically, in a subcortical "global best estimate buffer," for purposes of optimizing moment-to-moment serial behavior. The nested format of this buffer renders its contents conscious. I have conjecturally assigned this multimodal buffer to a region of unique connectivity in the dorsal pulvinar, where unit activity shows more selectivity for stimulus awareness than cortical visual areas assessed with the same flash-suppression method (see Wilke et al. 2009).

From this perspective, the irreducible requirement for olfactory stimuli to reach awareness is that olfactory information reach this pulvinar territory. Does it? The short answer would seem to be yes, and rather directly at that. In the monkey, the olfactory bulb itself projects to at least eight separate telencephalic territories. One of these is the anterior entorhinal cortex (Carmichael et al. 1994). Entorhinal cortex in turn is reciprocally connected with the relevant portions of the pulvinar (Insausti et al. 1987; Saunders et al. 2005). From this perspective at least, olfactory anatomy does not pose any insuperable obstacles to consciousness theory. I thank Ezequiel Morsella and colleagues for providing an opportunity to make these remarks on conscious olfaction.

\section{The primary (dis)function of consciousness: (Non)Integration}

\author{
doi:10.1017/S0140525X15002198, e189
}

\section{Liad Mudrik}

School of Psychological Sciences and Sagol School of Neuroscience, Tel Aviv University, Tel Aviv, 69978, Israel.

mudrikli@post.tau.ac.il

http://www.mudriklab.tau.ac.il

Abstract: Morsella et al. put forward an interesting theory about the functions of consciousness. However, I argue that this theory is more about showing what is not the function of consciousness, and claiming that it does not integrate, than vice versa - as opposed to its proclaimed goal. In addition, the question of phenomenality and its relations with integration is still left open.

The target article by Morsella et al. starts with a fundamental question, sought after by generations of scholars: "What does consciousness contribute to the functioning of the nervous system?" (introductory para. 1). To answer this question, it adopts an interesting and unconventional approach - the passive frame theorywhose main thrust is that consciousness "integrate[s] information ... involving incompatible skeletal muscle intentions for adaptive action" (sect. 2.4, para. 5).

Indeed, information integration has been repeatedly suggested as one function, and even as the primary function, of 
consciousness (e.g., Baars 2005; Dehaene \& Changeux 2011; Dehaene \& Naccache 2001; Koch \& Tononi 2011; Tononi 2013): Unconscious processing is typically claimed to rest on feedforward activations with little information sharing between processing nodes. Conscious perception, on the other hand, is held to involve long-range, recurrent processing and widespread neural activations that enable cortical interactions and synchronization (Baars 2005; Dehaene \& Changeux 2011; Engel et al. 1999; Lamme \& Roelfsema 2000; Schurger et al. 2015; Tononi 2013; Tononi \& Edelman 1998; Treisman 2003). These patterns of neural activation, then, are claimed to enable information integration - the combination of two distinct signals into a new, meaningful one. Under these accounts, consciousness is not only crucial, but also necessary for information integration (for review of these claims and empirical findings that either support or challenge them, see Mudrik et al. [2014]).

Despite the common use of the word "integration" and its description as the primary function of consciousness, the account suggested by Morsella and co-authors is utterly different from the above-mentioned theories: while for these theories, consciousness is almost omnipotent, as integration (and other functions) cannot take place in its absence, the passive frame theory portrays a somewhat impotent consciousness, lagging behind unconscious mechanisms, which actually do most of the work. As I argue below, it seems like the proposed account, albeit being innovative, comprehensive, and thought-provoking, is more about showing what is not the function of consciousness, and about claiming that it does not integrate, than vice versa-as opposed to its proclaimed goal.

According to the theory, the conscious field is clearly unintegrated in the sense that each conscious event is encapsulated from the others: it is discrete, devoid of any ability to interact or influence other states. Unconscious mechanisms, on the contrary, "combine" and "comprehend" the different contents in the conscious field in a way that leads to action plan selection. They are active, as opposed to the passive conscious states. To illustrate their point, the authors present the Internet analogy: Consciousness is the platform that allows two people - in analogy, some unconscious mechanisms - to debate, but it cannot resolve the conflict. It serves as means for transmitting the message, but has no bearing on the message itself, or its effects.

However, in this Internet analogy, where does the integration truly lie? Would one say that the Internet integrates the discussion, or that the two discussants, admittedly able to talk thanks to the Internet, are the ones doing the integration? By their own analogy - and the theory it aims at illustrating - the authors pull the rug from under their own concept of integration, in such a way that makes the latter devoid of real content. If unconscious mechanisms are the ones resolving action conflicts, what is the meaning of saying that consciousness's primary role is to integrate "incompatible skeletal muscle intentions for adaptive action"? Why not simply say that consciousness's primary role is to enable unconscious integration of incompatible intentions?

It seems as if the authors are trying to have their cake and eat it too: On the one hand, they ascribe all integrative functions to unconscious mechanisms. On the other hand, they keep saying that consciousness's primary function is information integration, perhaps reflecting their own reluctance to wave the consciousness-integration long-lasting connection goodbye. In that respect, their article could have started with the (very interesting) question, "What do unconscious processes contribute to the functioning of the nervous system?" - because their theory provides a clearer answer to this question, than to the one they chose to pose.

Aside from what may seem like confusion in goals (attempting to clarify the function of consciousness, while in fact arguing for the function of unconscious mechanisms and the unfunctionality of consciousness), the theory does not explain why the conscious field is needed for unconscious integration to occur. Without the Internet, our two discussants would not have been able to share their views. If so, consciousness may be needed for distinct unconscious events to share information. Why should it be phenomenal, though? In the Internet analogy, technology enables two intentional agents with phenomenal experiences to interact. Yet here, phenomenal states are supposed to enable the interaction of non-phenomenal states - which are claimed to interpret, resolve conflict, and yield decisions. Where does that leave intention? Why should information sharing be phenomenal? And why assume that non-phenomenal states are able to integrate, while phenomenal ones cannot interact with one another? These questions are left unanswered under the current account.

Finally, let's imagine that the creature in the cave is a human being, deliberating about whether he should run away from the smoke and leave all his precious belongings behind. What is the status of his conscious deliberations over this possible voluntary action, according to the passive frame theory? Do they represent false/illusionary states, given that (being conscious experiences) they cannot affect the chosen action, which is unconsciously determined?

To sum up, the present target article is an impressive attempt to put forward a comprehensive functional theory of consciousness. To that end, it uniquely combines together neuroscientific, psychological, and philosophical work, integrating current findings with ideas and suggestions that go decades back even to the nineteenth century - which by itself is a rare and important endeavor that should be applauded. What was the role of the authors' consciousness in forming such an integrative account? Did it only create perception-like representations of these ideas, so that unconscious mechanisms could then integrate them into a skeletomotor output of typing? My own (dysfunctional, nonintegrative?) conscious field wonders if this could indeed be the case.

\section{Conscious content generated by unconscious action-related adjustments}

doi:10.1017/S0140525X15002204, e190

\section{Farzaneh Pahlavan and Melana Arouss}

Laboratory of Social Psychology: Threat \& Society, EA 4471, Institut de Psychologie, Université Paris Descartes, 92774 Boulogne-Billancourt Cedex, France.

farzaneh.pahlavan@parisdescartes.fr@melana.arouss@etu.

parisdescartes.fr

http://lps.recherche.parisdescartes.fr/

Abstract: As mentioned in the target article by Morsella et al., the motor program's contents as a whole do not influence each other in the conscious field; however, research shows that some of their generated aspects could be sensitive to the context of the behavior. Such motivational "contextcontamination" leads to voluntarily "turn off" of negative states. Taking place out of the conscious field, these adjustments would have critical influence on behavior, such as aggression.

Created by Schelling (1800/1978), Freud developed the concept of the unconscious mind in his tripartite model of mind, using the analogy of an iceberg to describe the three levels of mind: conscious mind at the top, unconscious mind at the base, and preconscious mind in the middle of the iceberg. Considered a primary source of human behavior, the unconscious mind, like an iceberg, is the most important part of the mind, hidden because it is unacceptable to the conscious mind (Freud 1915).

In spite of initial skepticism, the idea or modern notion of unconscious processes is now an important focus of psychology, in terms of automatic (Bargh \& Chartrand 1999), implicit processing of information (Greenwald \& Banaji 1995). However, empirical research in psychology has its own limits, often being subject to alternative interpretations. Indeed, empirical research of unconscious processes underestimates the roles of conscious processes 
Commentary/Morsella et al.: Homing in on consciousness in the nervous system

and their significant regulatory effects in processing unconscious contents. In addition, the view that unconscious processes control individuals' everyday experience underestimates the individual-context dynamic. Hence, as rich as the research of unconscious processes has become, interpretation of related results reveals some difficulties. For example, in the case of temporary accessibility, the assumption of privileged access to the mind of negatively valenced information (Bargh \& Pietromonaco 1982; Wegner \& Bargh 1998), such as aggressive words or stereotypes (Devine 1989), and their conceptual replications (Bargh 2014; Bargh et al. 1996; Chen \& Bargh 1997), is not clearly established. In fact, other studies found rather contextual limits of so-called privileged access (Baron et al. 1992; Bodenhausen et al. 1994; Carver \& Scheier 1999; Pahlavan \& Lubart 2005; 2007).

Assertions about automatic evaluation of aggressive stimuli and automatic aggressive-goal-pursuit can also be explained in terms of construal levels of elaboration of goal-directed behaviors. For Srull (1997), association between situational features and goals is possible if the goal and the situation are both elaborated at equivalent levels of abstraction. In this sense, exposure to negatively valenced stimuli should trigger anger as well as fear constructs as predicted by the cognitive-neoassociationistic model (Berkowitz 1993), activating two parallel behavioral tendencies (flight/fight). In fact, in terms of behavior adjustment, the results of numerous aggression studies show that individuals "fight" only when there is little or no chance for "flight" (Izard \& Ackerman 2000).

Considering adjustment as mere modifications of cognitive knowledge, for individuals living in a changing environment, expectation-reality matches are an essential life-task motivation in order to feel secure. Adjustment process may therefore bring expectation and reality into accord based on prior new insight of reality through attentional effortful control. As long as reality meets individuals' expectations, attention will bring pleasure; otherwise, displeasure (Posner \& Rothbart 2000; Schachter 1987). However, adjustment processes are highly context-dependent (Bjorklund \& Pellegrini 2002). Empirical research supporting these assumptions (Pahlavan \& Arouss 2015; Pahlavan et al. 2012) shows how negative situational characteristics could alter executive performance in persons with dysexecutive syndrome and in healthy adults, with consequences for aggressive tendencies, using a feedback paradigm (see Table 1).

The same results were found with healthy French/Syrian adults using an aversive noise. For all participants, increases in negative reactivity due to negative feedback or aversive noise were concomitant with an inability to focus individuals' attention on ongoing tasks.

Therefore, in addition to objective experiences, adjustment processes are accompanied by subjective experiences, based on individuals' prior knowledge of the matter of adjustment. The interplay among different cognitive processes with which new-information is processed, past-knowledge recalled, ... gives rise to different influences from past-present experiences, changing the conclusions drawn from same experiences.

An automatic/unconscious view of aggression follows quite similar conceptual logic. However, as mentioned by Wegner and Bargh (1998), even in the unintended, unaware, difficultto-control or -inhibit situational influences on behavior investigated in classic social psychology, it is believed that individuals successfully control some situational aspects. Trying to respect their implicit contract, participants consciously deal with felt conflicting pressures. Other studies, relevant to research on heuristic processing of aggression (Zelli et al. 1995; 1996) demonstrate that individuals with chronic accessibility of aggressive constructs spontaneously interpret and encode the ambiguous behaviors as aggressive, unless they make deliberate inferences about the target's behaviors. This last finding suggests the inadequacy of measures of deliberate controlled inferences to reveal differences between aggressive/nonaggressive individuals (Todorov \& Bargh 2002). In line with these suggestions, other studies (Pahlavan et al. 2000a; 2000b) showed that compared to an individual performing long voluntary movements (30 $\mathrm{cm}$ ) of pushing/pulling cued by an aversive stimulation, those executing the same but shorter movements $(12 \mathrm{~cm})$ took more time for pulling than pushing. This negative priming reveals that the participants successfully ignored automatic movements triggered by aversive stimulation when they had enough time to deliberately control and adjust their voluntary movement (pulling instead of pushing).

For Bargh (2014), automatic processes may take-over the regularities in one's life. Unaware of the underling motives of their behavior, individuals fail to see that their biased behaviors are harmful to other people. Lack of knowledge about the true instigating cause (non-conscious) leads to rationalization and attribution of reprehensible behavior to socially desirable motives (Todorov \& Bargh 2002). In this view, aggressive behavior is considered to be a result of biased automatic control processes, without taking into account conscious regulatory processing of human aggression. This assumption ignores the results of the studies showing uniqueness of self-related processes and social interaction as a function of roles taken on by individuals in their social relationships (Andreou 2000; Boulton \& Smith 1994; Smith et al. 1993; Sutton \& Smith 1999). Hence, although there is no doubt that contexts automatically cue behavior, actions such as aggressive behavior implying mental representations of "Self" can occasionally be highly adaptive, and rarely unconscious (Cross \& Markus 1990; James 1890/1983).

The use of the construct "automatized unconscious goals" risks leading to some dangerous reductionism in viewing behavior. People often pursue multiple goals (Carver \& Scheier 1999), and it is natural to assume that some of them result from automatic processes cued by situational features. At the same time, it is

Table 1. (Pahlavan b Arouss). Observed differences for global executive functions and aggressive tendencies (anger and aggression) mean scores in function of experimental condition.

Experimental Condition

Scores

Executive Cognitive

Function-global

Anger

Aggression

Feedback Paradigm

Failure $(n=30)$

$109.70 \mathrm{~s}$

37.90

0.317

Success $(n=30)$

$83.75 \mathrm{~s}$

26.90

0.017

Aversive Noise of $80 \mathrm{~dB}$

Aversive Noise $(n=60)$

$128.21 \mathrm{~s}$

32.70

0.239

Neutral Condition $(n=60)$

$71.88 \mathrm{~s}$

28.33

0.164 
natural to assume that these processes are occasionally consciously mediated, particularly those with potential significance for self. Thus, even though the experience of emotions is a necessary factor for keeping people in pursuit of their specific goals, affective, cognitive, and motivational processes of action control are engaged whenever decisions are self-relevant. Self-knowledge is a significant regulator of the individual's social perception and behavior, leading to more readily encoding ambiguous interpersonal situations as personal affronts, or to cognitive reconstitution of the event, in order to make reprehensible conduct personally and socially acceptable. Therefore, people do not automatically remove all expectation/reality discrepancies (Carver \& Scheier 1999); rather, human self-motivation relies on managing discrepancies by exerting proactive control as well as reactive feedback control. By setting themselves valued or challenging standards, they create states of disequilibrium and then mobilize their efforts to reach them.

\section{Human consciousness is fundamental for perception and highest emotions}

\section{doi:10.1017/S0140525X15002216, e191}

\section{Leonid Perlovsky \\ Psychology Department, Northeastern University, Boston, MA 02115. Iperl@rcn.com \\ http://www.leonid-perlovsky.com/}

Abstract: Have Morsella et al. examined the fundamentals of consciousness? An experiment by Bar et al. (2006) has demonstrated the fundamental aspects of conscious and unconscious mechanisms of perception. The mental representations are not crisp and conscious like the perceived objects are, but vague and unconscious. This experiment points to the fundamental function of the neural mechanisms of consciousness in perception. Consciousness is also fundamental for the highest emotions.

I very much appreciate the Morsella et al.'s desire to follow an example set by physics and begin "with the examination of the most basic, elemental instantiation of the phenomenon of interest" (sect. 1.3, para. 1). However, I would question how successfully this intent has been realized. A conceptually simple experiment by Bar et al. (2006) has demonstrated the fundamental aspects of conscious and unconscious mechanisms of perception. The mental representations of even simple everyday objects are not crisp and conscious like the perceived objects are, but vague and unconscious. This experiment identifies and illuminates the conceptual framework, as well as the purpose and the fundamental aspect of the neural mechanisms of consciousness in perception (Perlovsky 2009).

Perception of events in the world is based on mental representations (Grossberg 1988; Kosslyn 1980). Adapting representations to reality therefore is a condition of survival. Correspondingly, humans have an instinctual drive for developing adequate representations. A theory of this drive (Perlovsky 2006; 2007; 2014a; 2015) is based on Grossberg and Levine's (1987) theory of drives and emotions. According to Grossberg and Levine, the mechanisms of drives include biological mechanisms similar to sensors measuring vital bodily parameters. If a vital parameter is outside of its safe range, neural signals indicate this to the decision-making parts of the brain. These neural signals and corresponding states of mind are perceived internally as emotions. The extension of this theory to developing representations (Perlovsky 2006; 2007; 2014a; 2015) has suggested the existence of a mechanism measuring similarities between mental representations and events in sensory data, or more generally, similarities between bottom-up and top-down signals. This mechanism is called the "knowledge instinct"; its neural mechanism - involving dorsolateral prefrontal cortex, orbitofrontal cortex, striatum, opioids, and dopamine - is outlined in Levine (2012).
The fundamental aspects of conscious and unconscious mechanisms of the neural operations of the knowledge instinct are characterized by a process of "from vague to crisp" representations. This has been confirmed in Bar et al. (2006), which identifies the neural mechanisms of consciousness in perception (Perlovsky 2009). Bar et al. have demonstrated that a process "from vague to crisp" takes approximately $0.6 \mathrm{sec}$ (hundreds of neural operations), and the major part of this process is inaccessible to consciousness. Only in the moment when a representation matches an object's projection (from the retina) on the visual cortex does the representation become clear-crisp and conscious. The knowledge instinct operations become accessible to consciousness at the end of the unconscious process "from vague to crisp"; therefore, the conscious perception is a small part of the entire perception process (Bar et al. 2006).

Why does "simple" object perception require such a complex operation, involving conscious, unconscious, vague, and crisp representations? The answer to this question can only be understood after decades of mathematical modeling of this process, which reveal the role of the conscious and unconscious. Mathematical psychologists modeling the process of perception, as well as artificial intelligence engineers developing robots with visual perception abilities, have been failing since the 1950s. Recently, the past decades of failures have been understood to be due to the logical models employed. Only the logical part of the perception processes is accessible to consciousness and has inspired the development of these past mathematical models. However, because the vague processes are not accessible to consciousness, their operations have not been noticed or understood and have been missed in the mathematical models (Perlovsky 2001). As it turns out, the models required for matching bottom-up and top-down signals based on logic require a number of computations larger than the interactions between all of the elementary particles in the entire life of the universe (Perlovsky 1998).

This complexity is a fundamental fact related to the Gödelian inconsistency of logic (Perlovsky 2013c), which is considered to be one of the most fundamental mathematical results of the twentieth century. The complexity has been overcome when the representations and the entire processes of matching bottom-up and top-down signals are modeled as processes "from vague to crisp" described by dynamic logic (Perlovsky 2006; 2007; 2013c). This mathematical model has been confirmed in (Bar et al. 2006). Thus, unconscious "vague-to-crisp" processes are fundamental for perception, cognition, and the knowledge instinct; the clear-crisp and conscious part of these processes is only a small part. Let us repeat, the vague processes are not accessible to consciousness; if they were, perception and cognition would be mostly vague. The unconscious is needed so that we are not aware of the useless vague perceptions. Crisp and clear ("logical") perception is a minor part of the perception process, but it is conscious (and therefore it biases our understanding toward logic); in our consciousness, we perceive a clear form of logic-like perceptions.

The knowledge instinct is similar to other drives in that its satisfaction is accompanied by emotional neural signals and states. These emotions related to knowledge are aesthetic emotions (Perlovsky 2014a). At lower levels of the mental hierarchy (perception of everyday objects), they are usually below the level of consciousness. At higher levels (cognition of abstract concepts), they could be conscious (existence of these emotions has been demonstrated experimentally in Perlovsky et al. [2010]). Representations near the top of the hierarchy model an entire life's experience; they are mostly vague, unconscious, and vaguely perceived as the "meaning of life." But the corresponding emotions could be conscious and perceived as emotions of the beautiful (Perlovsky 2010a; Schoeller 2015; Schoeller \& Perlovsky 2015). Usually, these are not well understood because of confusion between unconscious cognition and conscious language (Perlovsky 2013a; $2013 \mathrm{~b})$. The knowledge instinct and the related aesthetic emotions explain the strong effects of musical emotions, their 
origins, their evolution, as well as their functions in cognition (Perlovsky 2010b; 2012a; 2012b; 2014b). The function of musical emotions is to help overcome cognitive dissonances and to keep contradictory cognitions in consciousness (Masataka \& Perlovsky 2012; Perlovsky 2015; Perlovsky et al. 2013).

In summary, the knowledge instinct addresses aspects of consciousness from the conscious perception of objects to the highest forms unique to humans, including the concepts of the meaning of life, emotions of the beautiful, and musical emotions. I do not see how skeletal consciousness can explain the functions of consciousness and unconsciousness revealed in Bar et al. (2006), and specifically human functions of consciousness related to music and the beautiful. The knowledge instinct is fundamental for survival in humans and animals, which have used mental representations for perception likely since amniotes onward. Skeletal consciousness does not seem to be specifically characteristic for humans or higher animals.

I would conclude that the analysis in the target article is far from straightforward; instead, it is convoluted and disguises rather than reveals the conceptual framework, the purpose, and the mechanisms of consciousness.

\section{Homing in on consciousness: Why is a dream conscious?}

doi:10.1017/S0140525X15002228, e192

\section{Helene Sophrin Porte \\ 34 Robinson Parkway, Burlington, VT 05401. \\ hsp2@cornell.edu}

\begin{abstract}
Morsella et al. argue convincingly that consciousness is for adaptive voluntary action. What, then, is consciousness in a dream for? Two prior questions present themselves. In a dream, how do contents get into the conscious field? What are the properties of consciousness in a dream?
\end{abstract}

To the creature in the cave, consciousness is for adaptive "voluntary" action. What is consciousness in a dream for?

How do contents get into a dream? Rapid eye movement (REM) sleep cancels wakefulness and blocks external afference. How do contents get into the conscious field? Morsella et al. argue that consciousness arises from configurations of unconscious afference to content generators. Afference is not only "bottom-up," but also may include "top-down processes from knowledge systems and from frontal control regions" (sect. 4.1, para. 1). I shall argue that although (pre)frontal control regions are quiescent in REM, and afference is necessarily intrinsic, dream content is generated in the same way that Morsella et al.'s wakeful content is generated.

I have argued (Porte 2013) that REM afference implicates acetylcholinergic cell groups Ch1-Ch8 (characterized, for example, by Schaffer et al. 1998), and that REM afference, like retinal waves, is at once probabilistic and structured (for a review of retinal waves, see Wong 1999). Excitation evolves stochastically: afference to REM content generators is neither bottom-up nor top-down, but distributed on the neural axis.

What is consciousness in a dream like? In the conscious field in REM as in wakefulness, the liaison between action and consciousness is fundamental. The wakeful field is for skeletomotor action. In REM, action is in the field. Afference to skeletomotor neurons is real. Dreamers walk, run, swim, and fly. They rotate, accelerate, and run backward. They lie still only because REM, while allowing the eyes to move and the dreamer to breathe, paralyzes the skeletal muscles.

Grillner et al. (2008) state that "in all vertebrates, networks coordinating the basic propulsive movement synergy are located at the spinal level, whether in fish swimming, bird flight or mammalian locomotion" (p. 3). If when awakened from a dream Morsella et al.'s creature in the cave were to say, "I am walking down the street," most of us would agree that this conscious content might originate in a low-level' reticulospinal mechanism. Even frenzied dream action might exceed "I am walking" only in regard to level of excitation:

Hilary-Ashby and others walk, or walk-float rapidly in front of us-i.e., walk without effort or apparent stepping. Separately, we intone "hello," in rich sustained notes ... This catches on, and everyone ... begins to sing "hello." I sing out above the other voices, and begin to leap off the floor vertically-as if lifted, or "beaming up" - as I sing and bellow "hello" in an increasingly high (and difficult for me) register. I spring (again, as iffloating or flying up rapidly, vertically) so high that I nearly reach the extraordinarily high ceiling ... I feel queasy and afraid, briefly, but close my eyes and decide there is nothing to do but wait for the fall back to earth. (Anonymous dream report, 1984. Laboratory of J. Allan Hobson, Harvard Medical School.)

If "the command regions for locomotion are evolutionarily conserved and stimulation of these regions gives rise to walking, trotting or galloping in tetrapods like cats, depending on stimulation strengths [and if] stimulation of the same region in a bird gives rise to walking and at higher strengths flapping movements of the wings" (Grillner et al. 2008, p. 3), then this dream's "hellos" become mere, albeit calamitously aroused, vestibular incantation.

If configurations of afference that deliver content to the conscious field are action-related, then dream action should approximate wakeful action. Indeed, as Morsella et al. remark, "few would argue about the isomorphism among the conscious contents experienced while acting (e.g., saying 'hello') dreaming (e.g., saying 'hello' in a dream), or observing the action of another (e.g., hearing 'hello')" (sect. 3.1, para. 3). Likewise, the dreamer's point of view should be egocentric (sect.4.2, para. 3). The "hello" dream is very much in the (locomotor-vestibular) first person. To the creature in the cave, "I am walking" will afford a sense of self, in REM as in wakefulness.

The entrance into consciousness in REM is automatic. As Morsella et al. argue for wakefulness, content is "just there." Often, action is ongoing as a dream report begins. Or action "just starts," as if the unconscious induction of locomotion coincides with the emergence of conscious content. "Reflex-like" entrance of action into consciousness in REM accords with the operation of skeletomotor neural networks: "In most cases these networks are silent at rest and need to be activated from the brainstem command centres ... which via reticulospinal neurons regulate the activity level of the spinal [pattern generators]" (Grillner et al. 2008, p. 3).

Conscious contents in REM, like those in wakefulness, are encapsulated. Assume that the "hello" dream report describes the contents of the dreamer's conscious field completely. Can skeletomotor afference account completely for those contents? Someone will say, "The 'hello' dream is not an action dream. It is an 'Icarus' dream.” But no metaphor is present in the conscious field, and neither is Icarus. "The field itself," as Morsella et al. say of wakefulness, "has no memory and performs no symbol manipulation" (sect. 6, para. 2). If Icarus is to be found anywhere, it is in unconscious afference from a system that knows about Icarus. Encapsulated, the conscious (vestibular) content does not. To paraphrase T. S. Eliot: The dreamer has the experience, but misses the meaning (Eliot 1943).

By homing in on consciousness, Morsella et al. have established a new vocabulary for homing in on consciousness in sleep. Thus, we can say that in REM sleep the conscious field is not "for" adaptive skeletomotor action, although unconscious configurations of afference may be. We realize that in dreams that are not lucid, "framing" is explicitly absent. We see that the conscious field in REM and the wakeful conscious field share formal characteristics.

Whether consciousness in REM is for anything remains uncertain. But Morsella et al. help us see that the liaison between action and consciousness often verges on identity in REM - that at some level ("lower" than wakefulness?), skeletomotor action is consciousness. 


\section{Explaining consciousness: From correlations to foundations}

doi:10.1017/S0140525X1500223X, e193

\author{
Wolfgang Prinz \\ Department of Psychology, Max Planck Institute for Human Cognitive and \\ Brain Sciences, 04103 Leipzig, Germany. \\ prinz@cbs.mpg.de \\ http://www.cbs.mpg.de/staff/prinz-10359/@@index.html
}

Abstract: What does it take to explain the roles of consciousness for action and action for consciousness? This commentary claims that efficient functional explanations must meet two epistemological requirements: independent description of explanandum and explanans, and foundational explanation of their mutual relationship. It is argued that Morsella et al.'s target articledoes not fully meet these requirements.

The target article by Morsella et al. promotes an ambitious project, meant to explain both what consciousness is and how it subserves voluntary action. This commentary discusses some conceptual issues involved in such functional explanations, pertaining to levels of explanation, languages of description, and requirements of efficient description and explanation.

Functional explanations claim that $\mathrm{X}$ is for $\mathrm{Y}$, invoking a means/ ends relationship between the two: structure $\mathrm{X}$ serves as means for realizing function Y. To living systems, this scheme can be applied at two levels: operation and design. At the level of system operation, it applies to interactions between means and ends - one subserving the other (i.e., X subserves Y). At the level of system design, it applies to interactions in the reverse direction - one shaping the other (i.e., $\mathrm{Y}$ shapes $\mathrm{X}$ ). The first form of explanation applies to short-term operations in ongoing activity, the second to long-term design in development and evolution. Full functional accounts may combine the two scales. For instance, the claim that legs are for locomotion means that (1) evolution has designed legs to accommodate the function of locomotion so that (2) the ensuing design enables legs to subserve that function in ongoing operation. Likewise, the target article claims (i) that consciousness is designed to accommodate the needs of action control (ii) so that consciousness subserves action control in ongoing operation. Notwithstanding the interchange of roles of explanandum and explanans, the two explanations can be legitimately combined because they pertain to different levels and timescales.

When we talk about legs and locomotion, we address both $\mathrm{X}$ and $\mathrm{Y}$ in the common language of kinematics and dynamics. However, such use of a common language does not apply when discussing relationships between consciousness and action control. Consciousness pertains to phenomenal experience, whereas action control addresses behavioral performance and underlying brain mechanisms. Because the languages of experience and performance are incommensurate, there is no obvious way of bridging the categorical gap and explaining one through the other. A project aiming at "homing in on consciousness in the nervous system" promises a new attack on the mind/brain problem and the two-language problem entailed in it.

A convenient way of addressing this problem is to look for features that can be expressed and understood in both languages (Prinz 1984). Thus, to understand how consciousness can subserve action control in ongoing operation requires being able to discern features of conscious experience that translate into the language of performance. Likewise, to understand how the requirements of action control can shape consciousness in development and evolution requires ascertaining features of performance that translate into the language of experience. Such feature overlap may then lay the ground for foundational explanations (see discussion below).

Efficient functional explanations must meet two basic requirements: independent description and foundational explanation. Independent description requires that $\mathrm{X}$ and $\mathrm{Y}$ are both characterized in an equal and independent manner. The target article is more elaborate on the signature of action-related performance than the signature of conscious experience. This asymmetry runs the risk of violating independence. Independence description requires the provision of an independent account of the explanandum in the first place, that is, an account of core features of consciousness that are independent of its alleged role for action control (e.g., phenomenal experience, subjectivity, aboutness, intentionality, etc., as known from classical debates on the nature of consciousness). What is offered, instead, are functional features that already derive from the invoked role of consciousness for action control (pertaining to integration of competing efference bindings). As a result, these features cannot explain much more than themselves, and "consciousness" becomes an empty concept. It stands for no more than "that which is required for information integration in action selection." Good old consciousness gets lost this way and becomes replaced by an operationally defined entity that lacks any surplus meaning.

Foundational explanation requires that $\mathrm{X}$ and $\mathrm{Y}$ become related to each other in a way that conforms to our understanding of efficient explanation (Prinz 2003a; 2012, Chs. 1 and 2). Of foundational explanations, we demand that they help us understand how consciousness enables efficient response selection and how the requirements of efficient response selection act to shape consciousness. Why is it that information integration for action selection precisely requires consciousness (and not something else), and why is it that consciousness precisely subserves integration for action selection as proper function (and not some other function)? As discussed, convincing answers to these questions must specify feature overlap between performance and experience, that is, common features that make sense in both languages. It is not easy to see how Morsella et al.'s passive frame theory can fulfill this requirement. There is no obvious way in which functional features pertaining to information integration in action control could overlap with any constitutive feature of consciousness so that one could imply or require the other. Accordingly, this theory fails to offer a foundational account of consciousness (unlike, for example, self-representational approaches for which such overlap has been claimed; cf. Graziano 2013; Prinz 2003a; 2012).

While foundational explanations require conceptual overlap between $\mathrm{X}$ and $\mathrm{Y}$, correlational explanations may switch language by relating an experiential state such as consciousness to a computational state such as action conflict or a neural state such as activation in a corresponding brain network. However, correlational explanations do not answer the foundational question of how these relationships work (Graziano 2013, Ch. 1). To know that action selection is associated with, or even requires, consciousness (in correlational terms), does not mean to understand (in foundational terms) how the two are interrelated. We just acknowledge the miracle that they enable and require each other, without understanding how they do it. To understand how the magic trick is done, we need to move from correlations to foundations.

\section{Four questions for passive frame theory}

doi:10.1017/S0140525X15002241, e194

David A. Rosenbaum

Department of Psychology, Pennsylvania State University, University Park, PA 16802.

dar12@psu.edu

https://sites.google.com/site/rosenb13/

Abstract: Four questions are raised about the passive frame theory of Morsella et al.: (1) What is the relation of the theory to the responseselection-bottleneck view of attention? (2) Does the theory accommodate the contents of consciousness? (3) What about animals without skeletal muscles? (4) How do the contents of consciousness change with the development of automaticity?

I commend Morsella et al. for their attempt to come to grips with the most notoriously difficult question in behavioral and brain 
sciences: What is consciousness? To their credit, the authors offer a specific answer to this question that centers on the resolution of conflict between physical actions. I too have tried to understand mental activity in terms of internal competition (Rosenbaum 2014), but I have some specific questions for Morsella et al.

First, what is the relation of passive frame theory to Pashler's (1993) classic theory of attention, which places attention at the response selection bottleneck? Pashler's theory and the theory offered here are similar in this respect, though Pashler's theory mainly focuses on the amount of cognitive effort (or number of cognitive resources) that is summoned when responses must be selected, whereas Morsella et al. mainly focus on the qualitative aspects of experience related to response selection. How are the two views similar or different? Is passive frame theory an advance over Pashler's theory? If so, how?

Second, does passive frame theory actually or adequately address the content of consciousness? Although passive frame theory suggests that the need for consciousness is greatest when decisions must be made about skeletal muscle activity, can the authors defend the hypothesis that the contents of consciousness are limited to skeletal-muscle response selection, if indeed that is what they are suggesting? While it is conceivable that imagining oneself engaging in some activity boils down to anticipating the skeletal-muscle movements to be made at the time, the feelings of muscles pulling on bones are not what one typically experiences as one considers alternative action possibilities. To the extent that the contents of consciousness are typically far removed from the muscle sense, except perhaps when the physical demands of the task demand attention (Vallacher \& Wegner 1987), does the theory lose some of its power?

Third, is skeletal muscle activation really the defining ingredient for consciousness? Animals without skeletons engage in response selection all of the time. Think of octopuses, which can show learning of spatial layouts (Boal et al. 2000), can engage in astonishing feats of mimicry (https://www.youtube.com/watch?v=t-LTWFnG megdeception), and can learn by observing others (Fiorito \& Scotto 1992). Even the sea slug has neural circuitry that supports experience-based response selection (Kandel 2006). These invertebrates don't have skeletal muscles. Does that mean they lack consciousness? Perhaps I am putting too fine a point on the authors' claim that consciousness comes down to the deliberate control of skeletal muscles. Still, that is what they said, so it would be interesting to know what they think about consciousness in animals without striated muscles.

A fourth, more subtle question pertains to the fact that the sensory outcome of a given pattern of muscle activity (skeletal or otherwise) depends on the interplay of the body and the environment and is ultimately decided by the environment. Reaching out with the hand in one direction or the other has little consequence in and of itself but has a big effect if, say, the hand contacts one electoral ballot switch or the other. If the effects of muscle activation are far removed from the muscle activation itself, then the power of the passive frame theory claim seems weakened. Also, it turns out that muscle activations are never all-or-none. Graded activation of muscles, with more or less activation going to some muscles than others, is how the nervous system controls movement and physical stability (Rosenbaum 2010). Even when actors reverse course, switching from one target to another, detailed analysis of the movement trajectories they produce indicates that muscle activations change in a graded, continuous fashion rather than by abruptly turning off some muscles and turning on others (Henis \& Flash 1995).

Whether and how these kinds of graded muscle activations or their sensory consequences are represented in consciousness is an open question. Perhaps large-scale categorical differences between perceptual consequences of different actions are actually represented. Is that what Morsella et al. want to claim? If so, how do they think about the reduced need to imagine the perceptual consequences of alternative actions when choices between actions get practiced and become automatic? That is my fourth question: How do the contents of consciousness change with the development of automaticity? How do the authors conceptualize the growth of automaticity in their theory?

\section{Metacognition and conscious experience}

\author{
doi:10.1017/S0140525X15002253, e195
}

Bennett L. Schwartz and Ali Pournaghdali

Department of Psychology, Florida International University, University Park, Miami, FL 33199.

bennett.schwartz@fiu.edu apour005@fiu.edu

www.bennettschwartz.com

Abstract: Morsella et al. focus on the conscious nature of sensation. However, also critical to an understanding of consciousness is the role of internally generated experience, such as the content of autobiographical memory or metacognitive experiences. For example, tip-of-the-tongue states are conscious feelings that arise when recall fails. Internally driven experiences drive us to action and therefore are consistent with the current approach.

In the 1999 movie The Matrix, the mentor Morpheus tells the hero Neo that he has an advantage over his artificial-intelligence enemies because "their strength, and their speed are still based in a world that is built on rules. Because of that, they will never be as strong, or as fast, as you can be" (Wachowski \& Wachowski 1999). The assumption made by the characters in The Matrix and by Morsella et al. in the target article is that consciousness has a purpose, and that purpose is the flexibility of responding to a complex world. Just as Neo can bend the rules that define the virtual reality of the "Matrix," Morsella et al claim that consciousness allows us to over-ride fixed patterns of behavior, particularly when two or more of these patterns come into conflict. We applaud Morsella et al. for such a large-scale integrative theory on the function and neural basis of consciousness. We agree that there are substantial data that point to a role for conscious processing in flexible responding. However, we assert that a more expansive view of conscious experience must be considered than one that explains only sensation. In particular, we think that internally driven experiences, such as memory retrieval and metacognitive feelings, are critical in understanding consciousness.

Morsella et al. are explicitly interested in one category of conscious experience - namely, sensations. However, a theory of consciousness should consider other types of phenomenological experiences as well, such as propositions, attitudes, volitions, emotions, and feelings (see Kim 2010). For example, my hope that person $\mathrm{X}$ will be elected President and my belief that the events in The Matrix are not true are certainly conscious experiences, but something quite different from the sensation of green when I look at a tree. In particular, experiences associated with memory represent an important aspect of consciousness. Similar to the arguments made by Morsella et al., many aspects of memory, including the process of retrieval itself, remain opaque to conscious introspection, but the products of retrieval are vividly conscious.

Our claim here is that internal conscious feelings play an important role in conscious experience. That is, internal conscious feelings allow us to respond to error and conflict, just as conscious perception allows us to override automatic responses. Consider internal conscious experiences, such as tip-of-the-tongue states and déjà vu experiences (Schwartz \& Cleary 2016). These experiences are certainly conscious, although they derive from internal processing rather than the processing of external stimuli. Indeed, many metacognitive experiences - from confidence in retrieved answers to feelings of warmth about impending problem-solving - are conscious experiences (Kostic et al. 2015; but see Kornell 2014). This is not to say that some forms of metacognition may be implicit and operate at a non-conscious level (see Kornell 2014; Reder 1996), 
but many metacognitive experiences are not just conscious but attention demanding (Schwartz \& Metcalfe 2011).

Tip-of-the-tongue states are metacognitive experiences associated with difficulty in retrieval from semantic or lexical memory (Schwartz \& Metcalfe 2011). Neuroimaging data suggest that tipof-the-tongue states emerge from prefrontal cortex, particularly the dorso-lateral prefrontal cortex and the anterior cingulate (Maril et al. 2001; 2005). Similarly, other metacognitive experiences also arise in the prefrontal lobe (Metcalfe \& Schwartz 2016). This pattern contrasts with the view of Morsella et al. that the frontal lobe is less involved in conscious experience than other cortical regions. It may be that conscious sensations are a function of activity in cortex other than frontal, but that conscious internally driven experiences, such as tip-of-the-tongue states, derive from activity in the prefrontal regions. If this is so, it would appear unlikely that consciousness is predicated on any one area of the brain.

Similar to the arguments put forth by Morsella et al., we have argued that metacognitive experiences also serve the purpose of error detection - that is, in serving as alerts as discrepancies in the internal cognitive processes that drive memory (Metcalfe \& Schwartz 2016; Schwartz \& Cleary 2016). In tip-of-the-tongue states, the retrieval process has not succeeded, but the metacognitive process indicates that the unretrieved target is stored in memory. The tip-of-the-tongue state drives us to behavior (Schwartz \& Metcalfe 2011), much as the sensation of pain and heat drive us to put down the hot frying pan as quickly as possible. For example, people in tip-of-the-tongue states are more likely to express curiosity and actively seek out the answers to unretrieved questions than those not in tip-of-the-tongue states (Metcalfe \& Schwartz 2016). Moreover, people in tip-of-the-tongue states are likely to spend more time attempting retrieval than those not in tip-of-the-tongue states (Metcalfe \& Schwartz 2016). Thus, in metacognitive parlance, tip-of-the-tongue states are conscious monitoring experiences that allow us to control an otherwise opaque retrieval process.

Morsella et al. consider sensation as the main source of information as input for consciousness and, consequently, focus on the idea of phenomenal consciousness. In contrast, to use Block's terminology, we assert that they focus instead on access consciousness, that is, those conscious processes that inform action and speech (Block 2009). The input to such decisionmaking routines need not have phenomenal qualities, so their arguments beg the question as to why we have phenomenal consciousness at all. This problem is potentially resolvable by considering inner states (such as metacognition) as other sources of information for consciousness. These inner states can add subjective feeling to the access consciousness resulting from sensory information (see Koriat 2007).

In conclusion, we agree with Morsella et al. that exploring the function of consciousness will be critical in understanding what it is, how it evolved, and its neural correlates. However, restricting oneself to only one aspect of consciousness runs the risk of not finding the relations between domains of consciousness. Thus, we argue that any theory of consciousness must account for memory and metamemory experiences.

\section{Infer yourself: Interoception and internal "action" in conscious selfhood}

doi:10.1017/S0140525X15002265, e196

\section{Anil K. Seth \\ Department of Informatics, Sackler Centre for Consciousness Science, University of Sussex, Brighton BN1 9QJ, United Kingdom. \\ a.k.seth@sussex.ac.uk \\ www.anilseth.com}

Abstract: Can consciousness be understood through an association with voluntary skeletomotor action selection? Although flexible and integrated action selection is a plausible function for consciousness, a narrow focus on skeletomotor control neglects the contributions to conscious selfhood and subjectivity that rest on interoception and autonomic regulation (internal "action"). I consider these issues from the perspective of predictive processing.

Morsella et al. argue that consciousness serves as a frame that constrains and directs skeletal muscle output, by making encapsulated conscious contents available for adaptive voluntary control. Their argument builds in interesting ways on previous positions suggesting a role for consciousness in action selection (Merker 2005). However, they in some ways go too far, and in other ways not far enough.

The authors go too far in overstating the association between volition and consciousness, asserting that "the conscious field wholly and exclusively determines what in everyday life is called voluntary behaviour. Conversely, for every voluntary action, the organism can report a conscious content responsible for that action" (sect. 2.5, para. 1). This excludes the possibility that volitional responses can be affected by subliminal (non-reportable) stimuli, which we now know is not the case; for instance, voluntary decisions to withhold prepotent actions can be influenced by subliminal primes (Parkinson \& Haggard 2014). Also questionable are assertions that conscious perceptual content can be encapsulated from other factors like motivation (Balcetis \& Dunning 2010) and even action itself (Vishton et al. 2007).

More interesting, however, is where Morsella et al. do not go far enough. Despite their emphasis on the body, they do not consider interoception or autonomic regulation (internal "action"), which may be highly relevant to basic instantiations of conscious selfhood and subjectivity. This idea makes most sense from the perspective of the "Bayesian brain" or "predictive processing" (Clark 2013; Hohwy 2013; Seth 2013).

The basic idea of predictive processing is that perceptual content is determined by the brain's "best guess" of the (hidden) causes of its noisy and ambiguous sensory signals, computed according to Bayesian principles. Thus, visual perceptual content is determined by probabilistic inference of the causes of visual sensory signals, and so on for other modalities. Importantly, minimization of prediction error signals can be accomplished either by updating the prediction (corresponding to perceptual inference) or by performing actions to change sensory data-for example, by making eye movements to confirm sensory predictions. The latter process has been called active inference (Friston et al. 2010).

This perspective generalizes easily to other domains of perception and action (see my Fig. 1). Perception of body configuration and motion can be understood as proprioceptive (and kinaesthetic) inference. Indeed, skeletomotor actions themselves can be understood and modelled as minimization of proprioceptive prediction error through active inference (Friston et al. 2010). Active proprioceptive and kinaesthetic inference may also give rise to "action awareness," which refers to consciousness of action, in contrast to awareness of the consequences of actions or of action options (Seth et al. 2016). Classical examples here include experiences of intention and agency, which can be operationalized through paradigms like intentional binding (Haggard et al. 2002b).

In just the same way, perception of the internal state of the body-interoception - can be understood as inference on the (hidden) causes of interoceptive signals, with the resulting perceptual content emerging as conscious emotional or feeling states (Seth 2013). This framework of "interoceptive inference" generalizes previous two-factor appraisal theories of emotion (Schachter \& Singer 1962) to a rich multi-layered hierarchical model of the neural basis of emotion and physiological regulation.

Active inference in this context means suppression of interoceptive prediction errors through engagement of autonomic reflexes (internal actions). Interoceptive active inference is particularly significant for an organism because of the fundamental importance of maintenance of physiological homeostasis: It is more important for an organism to maintain its essential physiological variables within 


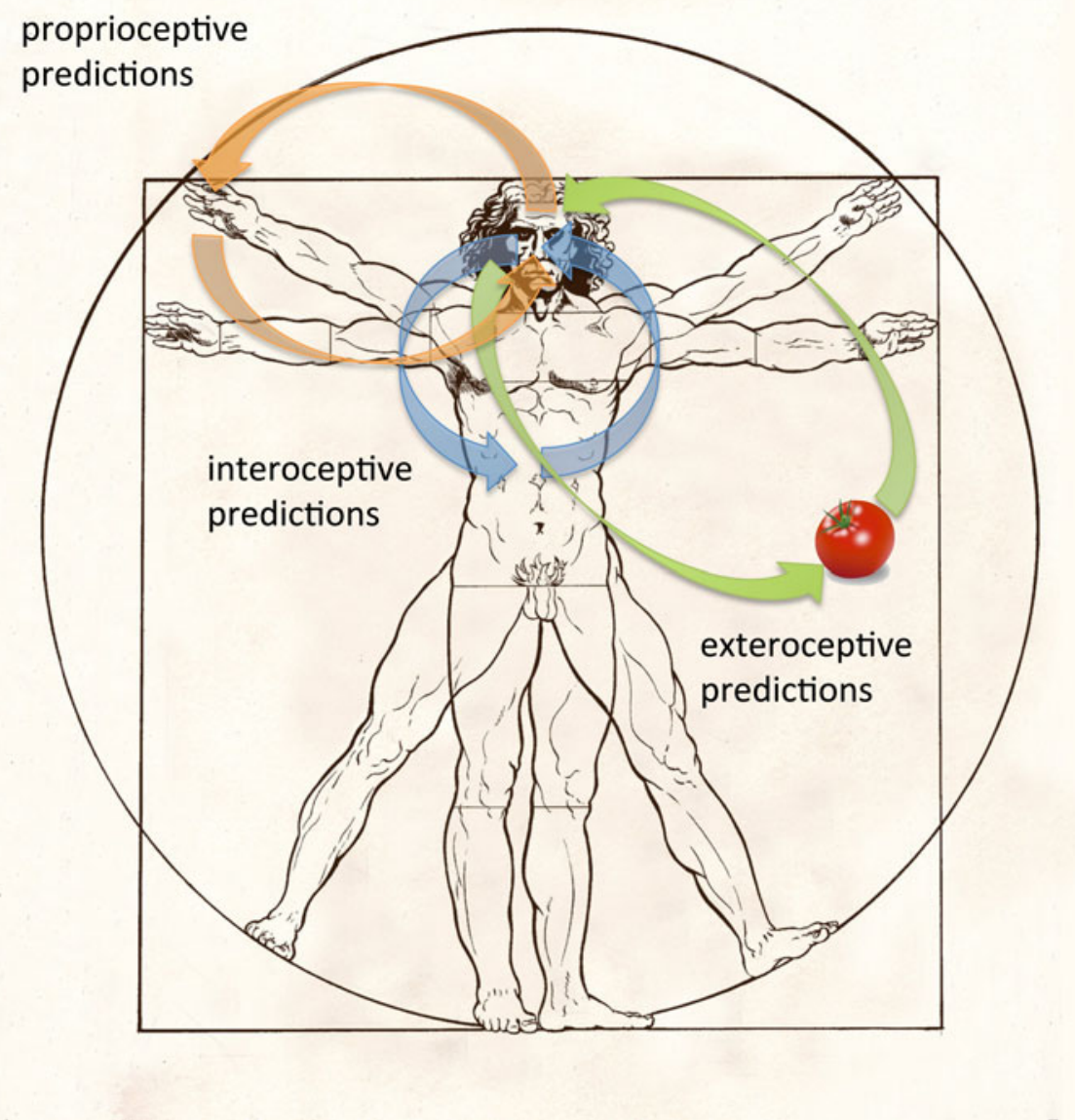

Figure 1 (Seth). Inference and perception in different dimensions, supported by reciprocal flows of perceptual predictions and sensory prediction error signals. Reprinted with permission from Seth (2015).

viable bounds, than it is to induce a detailed perceptual model of the internal milieu (Seth 2015), though the one depends on the other. This perspective echoes Karl Friston's influential "free energy principle" (Friston 2009) in saying that avoidance of atypical events (i.e., homeostatic regulation) necessitates a generative/predictive model of the causes of sensory inputs. It also recalls an older view from the largely neglected literature of cybernetics, where Ashby and Conant argued that "every good regulator of a system must be a model of that system" (Conant \& Ashby 1970, p. 89; see also Seth 2015).

From this perspective, internal actions make strong contributions to (affective) conscious contents through constraining interoceptive inference in the service of physiological homeostasis. These conscious feeling states may then further constrain adaptive skeletomotor responses when action options are evaluated with respect to their consequences for homeostatic integrity (see Damasio [1994] for an early non-Bayesian expression of this idea). Taking things further, combined multimodal or amodal inference across exteroceptive, proprioceptive, and interoceptive domains - geared towards the active regulation of homeostatic integrity - may underlie basic non-conceptual experiences of embodied selfhood and subjectivity (Seth 2013; 2015); see my Figure 1. Notably, because the preservation of physiological integrity is the most basic duty of any nervous system, this view is arguably more basic and "low level" than passive frame theory.

In summary, conscious contents can be usefully understood from a functional perspective in shaping adaptive voluntary skeletomotor control, just as Morsella and colleagues suggest in their passive frame theory. But human (and likely nonhuman) consciousness has many phenomenological dimensions which may shape perception and behaviour in many different ways. To explain basic experiences of emotion and embodied selfhood, I believe it is necessary to account for the role of internal actions (autonomic regulation) in actively maintaining physiological homeostasis. A Bayesian perspective provides a powerful framework for integrating these different dimensions of prediction and control, and opens exciting experimental opportunities for exploring the biological mechanisms underlying consciousness and self.

\section{Action-based synthesis of parental brain consciousness}

doi:10.1017/S0140525X15002277, e197

\begin{abstract}
James E. Swain, ${ }^{a-e}$ Ilinca Caluser, ${ }^{b}$ Zainab Mahmood, ${ }^{b}$ Madalyn Meldrim, ${ }^{\mathrm{b}}$ and Diana Morelen ${ }^{\mathrm{b}}$

${ }^{a}$ Department of Psychiatry and Behavioral Science, State University of New York-Stony Brook, Stony Brook, NY 11777, 'bepartments of Psychiatry, Division of Child and Adolescent Psychiatry, University of Michigan Health System, Ann Arbor, MI 48109; ' $P$ sychology, University of Michigan, Ann Arbor, MI 48109; ${ }^{\mathrm{d} C e n t e r}$ for Human Growth and Development, University of Michigan, Ann Arbor, MI 48104; ${ }^{\circ}$ Child Study Center, Yale University School of Medicine, New Haven, CT 06520.

jamesswa@med.umich.edu icaluser@umich.edu

zainabma@umich.edu meldrim@med.umich.edu

dmorelen@med.umich.edu

http://www.uofmhealth.org/profile/1678/james-edward-swain-md-phdfrepc
\end{abstract}

Abstract: Parenting consciousness, in line with passive frame theory, may be considered inseparable from action. With combined brain-imaging and 
Commentary/Morsella et al.: Homing in on consciousness in the nervous system

cognitive-behavioral analyses, we are in the early phases of understanding how parental brain circuits regulate parental thoughts and behavior. Furthermore, work on parental consciousness confirms the importance of motor outputs and outlines related circuits that inform consciousness across generations.

The concept of parent-infant attachment represents a landmark of contemporary developmental psychology (Bowlby 1969; 1973). From an evolutionary perspective, attachment represents an innate biological system promoting proximity-seeking between an infant and a conspecific attachment figure. This proximity, rooted in the thoughts and behaviors of parents, then increases the likelihood of infants to survive to reproductive age. Over the last 15 years, brain imaging studies have probed for this parental brain consciousness in health (Swain et al. 2014b) and illness (Moses-Kolko et al. 2014) - even beginning to focus on fathers (Swain et al. 2014a) - based upon the notion that individual differences in parenting behaviors may be based on variations in neural responses to own versus other baby-cry stimuli. Healthy human mothers are likely to pick up and hold and speak to their infants in response to their infant's cry, and this complex of responsiveness is known to calm an infant (Esposito et al. 2013). Perhaps because of an evolutionary advantage, these parental motor responses are widespread across cultures (Bornstein et al. 1992), neurobiologically embedded (Kim et al. 2016), and are important to understanding consciousness.

Indeed, this work on human parental brain consciousness using own-baby-cry stimuli (Swain et al. 2004) has identified parts of the motor system, pertinent to the work of Morsella et al. in the target article. Indeed, parent brain responses to own versus other babycry have included at least two aspects of the motor systems: (1) the pre-supplementary motor area (pre-SMA, Broca's area, superior temporal lobes), known to be involved in the preparation for movement and conscious intention to move (Haggard 2008; Nachev et al. 2008), imagining to grasp (Filimon et al. 2007), experiencing an "urge" to move (Fried et al. 1991), and preparation for a voice production (Brendel et al. 2010); and (2) bilateral inferior prefrontal cortices (Broca's areas) associated with social speech processing (Horwitz et al. 2003). A recent study further suggested that motor responses to baby-cry may be a function of poverty (Kim et al. 2015) underlining the considerable plasticity in parental consciousness systems (Kim et al. 2010; 2014; 2016; Swain 2007) according to environment that is itself defined by parenting.

Increasingly sophisticated and ethologically sound stimuli have been used to activate a range of other brain areas that support sensorium to motor output aspects of consciousness. In one of these studies, mothers at 4 to 6 months postpartum were divided into two groups: mothers with highly synchronous maternal behavior scores and low intrusiveness scores (synchronous mothers), and mothers with low synchronous scores and high intrusiveness scores (intrusive mothers) (Atzil et al. 2011). Synchronous maternal behaviors, including coordination of gaze, touch, and vocalizations with infants, are interpreted as more sensitive parenting behaviors and are associated with positive infant outcomes. Contrariwise, intrusive maternal behaviors include lack of coordination and more directedness with the infant, and they tend to be associated with maternal anxiety and stress responses (Feldman 2007). During a neuroimaging session, mothers were presented with video clips of their own infants and an unfamiliar infant. The main contrast between responses to their own versus the unfamiliar infant was greater activation in the NAcc (Nucleus Accumbens), a key reward/motivation region, and the amygdala, a key stress and negative emotion processing area. When intrusive and synchronous mothers were compared, intrusive mothers showed greater responses in the amygdala to their own babies, whereas synchronous mothers showed greater activation in the NAcc. Furthermore, functional connectivity in the whole brain using the NAcc and the amygdala as seed regions was examined, and the intrusive and synchronous mothers were compared. In synchronous mothers, activity in the NAcc was correlated with activity in attention and social information processing regions, including the inferior frontal gyrus (IFG), the medial frontal gyrus, visual and motor areas, and the parietal cortex. Conversely, intrusive mothers showed greater connectivity between the amygdala and the orbitofrontal cortex (OFC), which is characteristic of elevated anxiety. Thus, reward-related neural responses to one's own infant were associated with enhanced neural connectivity for attention and social information processing, which, in contrast to anxious responses, may support synchronous maternal consciousness.

In another fascinating study of maternal empathy that connects sensory and motor output circuits, mothers observed and imitated faces of their own and other children (Lenzi et al. 2009). This study specifically examined the mirror neuron system, localized in the ventral premotor cortex, inferior frontal gyrus (IFG), and posterior parietal cortex, which activates not only when an individual performs an action, but also when observing someone else perform that action. Neural activation in the mirror neuron system was preferentially engaged in response to own child and was correlated with maternal reflective function-sometimes also referred to as mentalization.

Using own versus other baby-cry stimuli, the brain has been studied for possible association with observed maternal mental state talk (Hipwell et al. 2015). Prior to the functional magnetic resonance imaging (fMRI) brain scan, mothers were filmed in face-toface interaction with their 4-month-old infants, and maternal behaviors were blindly and independently coded. Higher functional activity in the right fronto-insular cortex to own versus other babycry at the group level, in addition to bilateral subcortical regions including the thalamus, amygdala, hippocampus, and putamen, was positively associated with mental state talk-though, interestingly, not with global aspects of observed caregiving.

Finally, a widespread set of brain responses was reported in a recent study of mothers responding to child visual feedback after a caring decision (Ho et al. 2014). Responses that correlated with dimensions of empathy included the amygdala, ventrolateral prefrontal cortex (PFC), and supplementary motor area that may also fit with broader work on altruism (Brown \& Brown 2015; Preston 2013; Swain et al. 2012) and critically inform progress on consciousness connecting sensory with motor output as aspects.

\section{ACKNOWLEDGMENTS}

The authors are supported by the Departments of Psychiatry, Psychology, Center for Human Growth and Development, and Injury Center at the University of Michigan, National Alliance for Research on Schizophrenia and Depression, Klingenstein Third Generation Foundation, NIMHD/NICHD RC2MD004767-01, Michigan Institute for Clinical Health Research and National Center for Advancing Translational Sciences UL1TR000433, and Robert Wood Johnson Health and Society Scholar Award.

\section{The science of consciousness must include its more advanced forms}

\section{doi:10.1017/S0140525X15002289, e198}

\author{
Andrew Vonasch, ${ }^{a}$ E. J. Masicampo, ${ }^{\text {b }}$ and \\ Roy F. Baumeister ${ }^{a}$ \\ ${ }^{a}$ Department of Psychology, Florida State University, Tallahassee, FL 32305- \\ 4301; ' Department of Psychology, Wake Forest University, Winston-Salem, \\ NC 27109. \\ vonasch@psy.fsu.edu masicaej@wfu.edu baumeister@psy.fsu.edu \\ https://psy.fsu.edu/ baumeisterticelab/vonasch.html \\ http://college.wfu.edu/psychology/about-the-department/faculty-and- \\ staff/e-j-masicampo/ \\ http://www.psy.fsu.edu/faculty/baumeister.dp.html
}

Abstract: Morsella et al. argue that science should not focus on high forms of consciousness. We disagree. An understanding of high forms of 
consciousness is invaluable to the scientific study of consciousness. Moreover, it poses challenges to the passive frame theory. Specifically, it challenges the notions that conscious thoughts are not connected and that consciousness serves skeletomotor conflict only.

Morsella et al. propose an elegant theory of consciousness. Consciousness, they argue, is a passive frame through which skeletomotor conflict is resolved. The authors ascribe much of the originality in their theorizing to a strategic focus on the most elemental aspects of consciousness. In keeping with their simple approach, Morsella et al. argue that science should not focus on high forms of consciousness. We argue, on the contrary, that an understanding of high forms of consciousness is indispensable to consciousness theory, and that such understanding offers invaluable insights into passive frame theory (PFT). In many respects, the latest theories about higher forms of consciousness, including our own (Baumeister \& Masicampo 2010), reinforce PFT. But in other ways, they pose challenges that PFT must overcome. In either case, we object to being told that science should not study the higher level.

Many theorists distinguish two levels of consciousness. Morsella et al. are concerned with the most basic form of consciousness, which we term phenomenal awareness (Block 1995a) and consider to be present in most if not all animals (Mendl \& Paul 2004; Panksepp 2005). Our own work focuses on the higher form of consciousness, which we termed conscious thought. Conscious thought is unique to humans (Damasio 1999; Edelman 2004) and has been linked to such capacities as self-consciousness, mental time travel, theory of mind, and logical reasoning. We argue that conscious thought is essentially a place where the mind simulates sequences of events in the service of participating in society and culture (Baumeister \& Masicampo 2010).

Understanding conscious thought is indispensable to a full understanding of consciousness. Morsella et al. seem in some ways to endorse this view. Though their theory is based on the simplest forms of consciousness, they use it to explain consciousness at the highest levels, as in their Thanksgiving dinner scenario. Hence, their theory should be compatible with the functions of conscious thought, even if conscious thought is not its main focus.

Our theory shares many features with PFT. Both theories argue that consciousness is the place where the mind integrates information, allowing for competing intuitions to be resolved. Moreover, both theories locate control in automatic and unconscious processes. Thus, as far as the suggestions from PFT that consciousness resolves motivational conflict and is passive, we are in agreement.

However, two tenets of PFT are incompatible with our analysis of conscious thought. The first is the notion that conscious contents are responses to environmental stimuli-not other mental stimuli. With this assertion, PFT "counters the everyday notion that one conscious thought can lead to another" (Morsella et al. target article, sect. 4.1, para. 6). This view is incompatible with the most basic understanding of conscious thought. We have argued that conscious thought is useful for mentally simulating sequences of ideas or events (Baumeister \& Masicampo 2010). Much of human thinking fits this pattern, especially including language, logical reasoning, and narrative storytelling. Crucially, the sequences that comprise conscious thought are not a random assortment of percepts triggered by external stimuli. Rather, conscious thoughts comprise meaningfully connected sequences of ideas. Conscious thought's most salient feature is that it is experienced as a never-ending stream, with new thoughts often flowing out of the previous one (e.g., James 1890). Each step in logical reasoning is partly but directly caused by the preceding thought.

The connectedness between thoughts is part of the efficacious power of conscious thought. By combining ideas into meaningful sequences, new and useful properties can emerge. A person who is planning when to go to the airport does so by working backwards from takeoff, subtracting away the time it takes to walk to one's gate, check in, take a taxi, and so forth. The end result is a useful and unforeseen bit of knowledge: the precise time at which one should get going. It was reached simply by processing information one already has. This would be an intractable problem if each conscious thought did not lead directly into the next. Most types of conscious thought, including storytelling, language use, perspective taking, and logical reasoning, hinge on a direct relationship between thoughts.

Our second issue with PFT is its assertion that all conscious processing is for resolving motor conflict. We argue that the integration that occurs in human consciousness serves a broader purpose: privileging certain responses over others, whether those are motor movements or attitudes, preferences, or ideas. In our view, Morsella et al. have captured the process of integration and conflict resolution quite nicely, but they place an unnecessary constraint on the process by linking it exclusively to motor conflict. It is quite likely that the unconscious selection process by which animals regulate motor conflict evolved to allow humans to regulate other sorts of conflicts (e.g., between ideas when thinking through complex problems). During many conscious thought processes, such as logical reasoning or language use, there is no apparent skeletomotor conflict. As a case in point, which muscle conflict was being resolved when Morsella et al. employed conscious reasoning to devise PFT? Creative psychological theories may of course influence behavior downstream, but they are not created to resolve momentary inputs into the skeletomotor system.

In sum, we see much to like about PFT. However, we object to the notion that the scientific study of consciousness should eschew its more advanced forms. As with any scientific inquiry, a full understanding of the problem is best served by approaching it from all sides. A functional theory of consciousness that omits some of its forms and functions will remain incomplete. Moreover, research on conscious thought poses challenges to PFT. Specifically, the notions that conscious thoughts are not connected and that consciousness only serves skeletomotor conflict need to be reconciled with the prevailing view of human consciousness - that conscious thoughts are connected in meaningful ways, and that they concern not just competing motor impulses, but conflicts that are deeper and more meaningful.

\section{Authors' Response}

\section{Passive frame theory: A new synthesis}

\author{
doi:10.1017/S0140525X15002812, e199
}

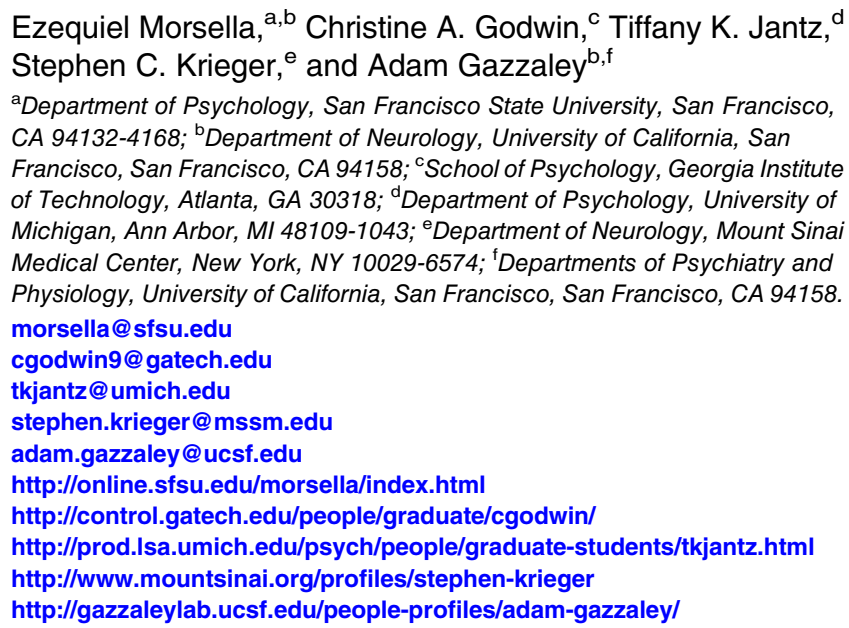


Response/Morsella et al.: Homing in on consciousness in the nervous system

Abstract: Passive frame theory attempts to illuminate what consciousness is, in mechanistic and functional terms; it does not address the "implementation" level of analysis (how neurons instantiate conscious states), an enigma for various disciplines. However, in response to the commentaries, we discuss how our framework provides clues regarding this enigma. In the framework, consciousness is passive albeit essential. Without consciousness, there would not be adaptive skeletomotor action.

\section{R1. The nature of the problem and definitions of consciousness}

For decades, the question of what consciousness contributes to the functioning of the nervous system has perplexed theorists and experimentalists, leading some of the greatest scientific minds, including the Nobel Laureates Leon Cooper, Francis Crick, Gerald Edelman, Eric Kandel, and Charles Sherrington, to conclude that answering this question is one of the greatest puzzles in science. As Shallice (1972) asserts, "The problem of consciousness occupies an analogous position for cognitive psychology as the problem of language behavior does for behaviorism, namely, an unsolved anomaly within the domain of the approach" (p. 383). Passive frame theory (PFT), a synthesis of diverse ideas, attempts to answer this question and yield novel insights about consciousness and the brain, all within a conceptual framework that, importantly, is internally coherent (Dux, Gainotti) and comprehensive (Mudrik). PFT attempts to illuminate what consciousness contributes to nervous function, how it serves this role, and what consciousness is, at least in mechanistic, functional terms. In the framework, consciousness is passive albeit essential. Without it, there would not be adaptive skeletomotor function. The theory does not address what Marr (1982) refers to as the "implementation" level of analysis, that is, how neural activities instantiate these conscious states, which is an enigma for various disciplines, including neurobiology. However, in response to the commentaries, we discuss how PFT provides clues regarding this enigma. In addition, we emphasize how the framework is different from established models.

Regarding the commentaries, we are grateful for their collegial, constructive, and thoughtful nature. We will study these commentaries for years to come. Several of them contain insights (e.g., tasting always requires a voluntary act [Gallo], action selection in dreams [Porte], and unique properties of olfaction [Merker]) that will serve as a basis for future research. Many commentaries also contained deep questions that can be answered only after years of further investigation. We were pleased that many commentators viewed PFT as a unique, novel, and internally coherent framework. In the following sections, we respond to the general themes raised in the commentaries.

With our EASE (elemental, action-based, simple, and evolutionary-based) approach, we focused on the most basic form of consciousness (e.g., the experience of a smell, visual afterimage, tooth pain, or urge to scratch an itch) and contrasted it with unconscious processes (e.g., the pupillary reflex, peristalsis). We avoided precise definitions of the phenomenon under investigation because, as noted by Sir Karl Popper, definition is the final stage, and not the beginning, of scientific inquiry. For scientific progress, one needs only identifications and contrasts (e.g., nausea vs. the pupillary reflex). A useful working definition for basic consciousness is provided by Nagel (1974), who proposed that an organism possesses basic consciousness if there is something it is like to be that organism - something it is like, for example, to be human and experience pain, breathlessness, or yellow afterimages. Similarly, Block (1995b) proposes that, "the phenomenally conscious aspect of a state is what it is like to be in that state" (p. 227). All of the contents of which one is conscious compose the conscious field, which changes over time (Fig. R1). The size of the field changes, in a sense, when a new content, which could stem from polysensory configurations of afference (e.g., the McGurk effect), becomes conscious (Fig. R2).

\section{R2. Explanatory power and novelty of passive frame theory}

Blackmore, Lin, Mudrik, and Prinz requested clarifications about the explanatory power of PFT. As a synthesis of six theoretical approaches from diverse fields of study (ideomotor theory, subset consensus, integration consensus, encapsulation, sensorium hypothesis, and PRISM [parallel responses into skeletal muscle]), PFT yields novel insights. For instance, our approach specifies how, when these hypotheses are united, then something resembling our proposed "frame" architecture (in which the skeletomotor response to one conscious content is framed by the other contents) would be required for adaptive action. PFT also explains subjective data from (a) intersensory conflicts, (b) smooth muscle conflicts (Morsella et al. 2009a), (c) synchrony blindness (see sect. R7), and (d) skeletomotor conflicts (e.g., holding one's breath). PFT also explains (e) why one is conscious of contents even when those contents are irrelevant to other contents or to ongoing

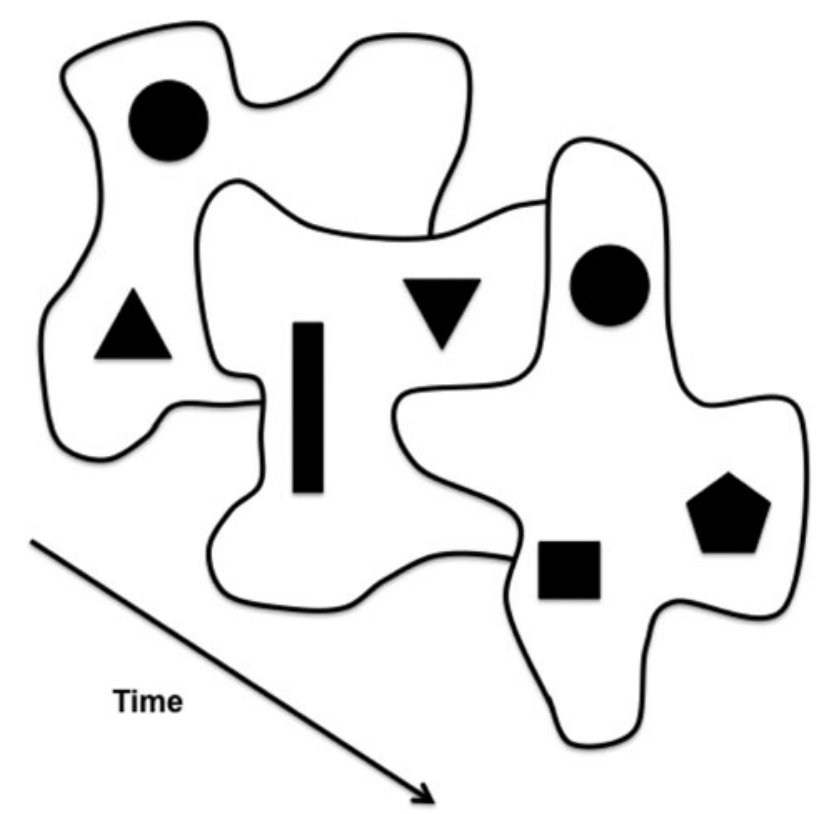

Figure R1 (Morsella et al.). The conscious field, with a different medley of conscious contents at each moment in time. Each of the three conscious fields, representing three different moments in time, possesses its own configuration of conscious contents (the filled shapes). One conscious content (e.g., the triangle) can be a sound; another conscious content (e.g., the square) can be an olfactory stimulus or an action-related urge. 


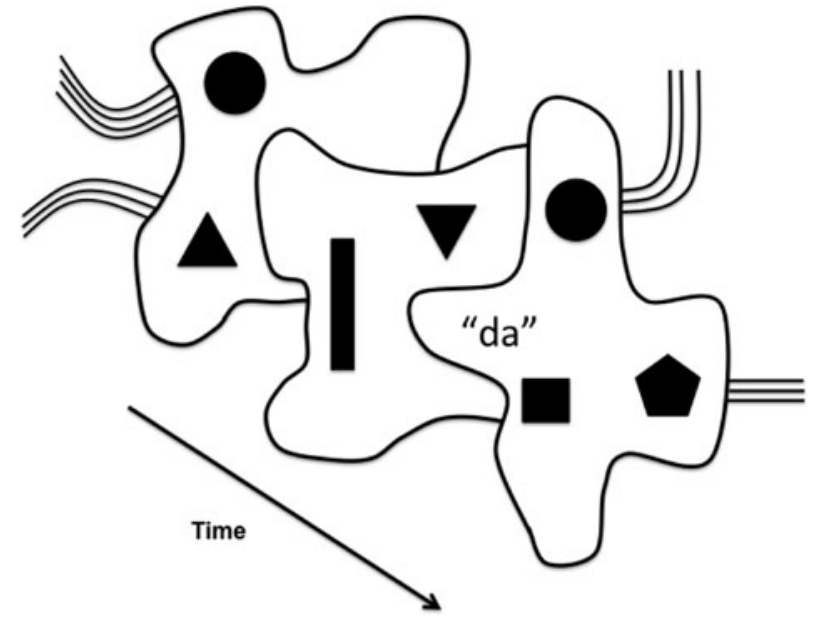

Figure R2 (Morsella et al.). The conscious field, with a different medley of conscious contents (filled shapes) at each moment in time. The conscious field during the third moment includes the percept "da," induced by the intersensory, McGurk illusion. Another conscious content could be a phonological representation (e.g., /haus/) triggered by seeing a word (e.g., "HOUSE"). The lines feeding into each conscious field represent the unconscious and often polysensory configurations of afference that are involved in the generation of each content.

action, and, relevant to Seth's comment regarding the relationship between consciousness and effector systems, (f) why skeletal muscle is the only "voluntary" muscle. Regarding (f), it is important to emphasize that the conscious acts of expressing (or suppressing) inhaling, blinking, swallowing, and micturating all involve, specifically, skeletal muscle. Accordingly, regarding digestion, one is conscious only of those phases of the process that require coordination with skeletomotor plans (e.g., chewing or micturating) and none of those that do not (e.g., peristalsis). Conversely, no skeletomotor plans are involved in the actions of consciously impenetrable processes such as the pupillary reflex, peristalsis, and stomach action, all of which involve smooth muscle. ${ }^{1}$ Thus, in response to Seth, the integration achieved through conscious processing is intimately related, not to perceptual processing, smooth muscle control, or motor control, but to skeletomotor action selection. Simply put, PFT explains that consciousness is for voluntary action. Without conscious mediation, adaptive integration fails to occur, as in the case of unintegrated actions (e.g., dropping a hot dish or failing to hold one's breath while underwater).

Many commentators have mentioned that the approach is novel. Others (e.g., Blackmore, Hommel \& Wiers) have requested more information regarding its novelty. (As a synthesis, PFT naturally contains ideas that have been presented elsewhere.) First, unlike other approaches, PFT specifies which integrations require consciousness and which do not, which is a current and major problem (cf. Mudrik et al. 2014). Several approaches posit that the integration associated with consciousness is for high-level semantic processes (Mudrik et al. 2014; Thagard \& Stewart 2014), which is at odds with our more "lowlevel," action-based proposal. There are also accounts in which consciousness is, not for intra-organismic processes, but for high-level, sociocultural interactions (Banks 1995; Carlson 1994; Frith 2010; Macphail 1998; Prinz 2012).
Other high-level accounts (e.g., Clark 2002; Koch 2004) propose that conscious processes are functionally unique because they tax semantic memory or top-down processes or are capable of anticipating the future. Moreover, some have hypothesized that consciousness serves no function in action control (Hommel 2013; Koch 2014; Masicampo \& Baumeister 2013; see also Jackson 1986; Kinsbourne 1996; 2000; Pinker 1997). (Here we are excluding mention of the many theories in which consciousness is epiphenomenal.) As is evident in the commentaries, some theorists (e.g., Blackmore) disagree with our basic assumptions that there is a difference between conscious and unconscious processing and that consciousness is associated with only a subset of nervous function.

Second, unlike other approaches, we propose that the integration provided by consciousness is associated, not with perceptual processing (e.g., afference binding), efference binding, or smooth muscle binding, but with binding for a peculiar kind of action control. Third, PFT is unique in specifying that conscious contents influence only action systems and not content generators: Conscious contents are sampled only by action systems. As far as we know, PFT, building on Morsella (2005) and Merker (2013c), is the only such account. PFT is also unique in that it focuses on olfaction instead of on vision and is actionbased instead of perception-based.

Moreover, PFT is unique in proposing that (a) conscious contents cannot influence each other either at the same time or across time, which counters the everyday notion that one conscious thought can lead to another conscious thought; (b) one conscious content does not "know," and should not know, of its relevance to ongoing action, to higher-order goals, or to other contents in the field; (c) though consciousness is not epiphenomenal or omnipresent (e.g., as in panpsychism), its role is more passive and less teleological than that of other accounts (e.g., Baars 1988; 2002; Dehaene 2014); and (d) during a frame check, the field functions as a unitary entity in terms of its influence over the skeletomotor output system. The latter, (d), is an important point that renders moot the debate concerning whether the conscious field should be construed as a unitary or componential entity (cf. Searle 2000): Action-related modules in the skeletomotor output system must treat, in terms of functional consequences, the mosaic of contents in the field as one thing. Last, (e) PFT reveals that, during action selection, anticipated action effects, actual action effects, and information about the immediate environment must exist as comparable tokens in a common decision-space. Although consciousness has historically been associated with the highest levels of processing, here it is revealed that consciousness must occur at the level of processing that is shared with that of representations of the immediate external environment (e.g., olfactory stimuli). The conscious field is most concerned, not about the future or past, but about the immediate present (Pahlavan \& Arouss), the scenario in which overt action will unfold. Last-minute changes in a course of action might arise from entry of new contents (Merker 2013c).

Hommel \& Wiers request that we differentiate PFT from "global workspace" approaches. Consistent with Baars (1988; 2002; see similar models in Anderson 1983; Minsky 1985; Selfridge 1959), PFT proposes that these states integrate nervous processes that are otherwise 
Response/Morsella et al.: Homing in on consciousness in the nervous system

independent. However, PFT is based more on Jamesian ideomotor approaches, which are action-based, than it is on workspace models. In the development of PFT, that which rendered it internally coherent was reconciling ideomotor theory with encapsulation: If one adopts the notions of encapsulation and of ideomotor processing, in which percepts must activate response codes (which also occurs in "continuous flow" [McClelland 1979] or "cascade" [Eriksen \& Schultz 1979] models), and if one accepts that intuitions about perception-and-action (as in the case of a blackboard diagram in which sensory inputs are connected to motor outputs) are actually computationally impossible (Tsotsos 1995; 2011), then it becomes apparent that something like our proposed frame is needed for adaptive action. Unlike the workspace models (e.g., Baars 1988; Dehaene 2014), which propose that conscious representations are broadcast to modules engaged in both stimulus interpretation and content generation, in PFT (as in Merker 2007), the contents of the conscious field are directed only at the unconscious processes of the skeletomotor output system (Fig. R3). The proposed architecture is consistent with the view (Cisek 2007; Cisek \& Kalaska 2010) that, in the nervous system, actional processes cannot be distinguished from decision-making processes (see Hardcastle, White, Kloos, \& Hardcastle [Hardcastle et al.]). Finally, unlike in workspace approaches, in which consciousness serves more than a handful of functions (e.g., Baars 1988; Dehaene 2014), we propose that the conscious field serves only one basic, passive role. It performs this same basic function for several kinds of processes, including some high-level functions (e.g., in adult humans). Figuratively speaking, in PFT, the real work is not done in the conscious field (Gur): The conscious field is a workspace without the work (Lashley 1956). In contrast, according to Baars (1988), consciousness serves many functions,

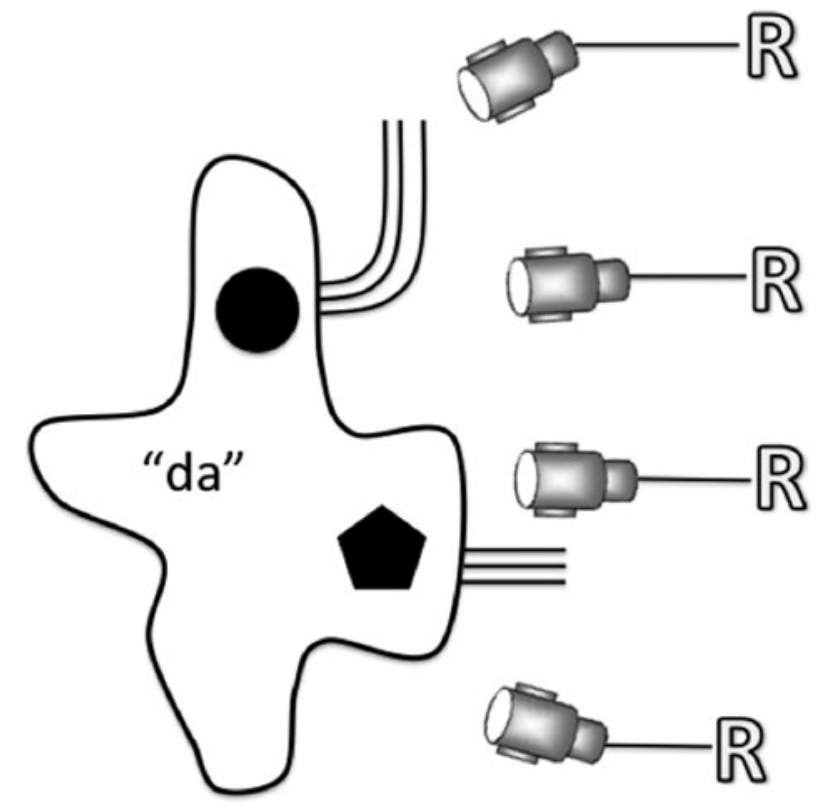

Figure R3 (Morsella et al.). At one moment in time, the conscious contents are apprehended by the unconscious, action mechanisms of the skeletal muscle output system. Each mechanism (represented by a gray sensor) is associated with a certain kind of (unconsciously mediated) action (e.g., articulating vs. reaching), which is signified by the Rs (for Responses). including adaptation and learning, decision making, analogy forming, editing and debugging, metacognitive self-monitoring, and autoprogramming.

\section{R2.1. Field construction and conscious versus unconscious integration}

In PFT, both the content generators and response systems are complex, unconscious processes, which is consistent with comments (Lin, Mudrik) about the sophistication of unconscious processes. It is clear that, in the construction of the conscious field, there is the participation of unconscious representations from both bottom-up and top-down sources, including those associated with frontal cortex (Bar et al. 2006; Perlovsky). As noted by Perlovsky, and by LeDoux (1996), some of these pre-conscious representations are "vague" and poorly developed compared to conscious contents (Perlovsky). The mechanisms of multiple drafts (Dennett 1991), apperception (Wundt 1902/1904), and reentrant processing involve unconscious afference from topdown and bottom-up sources (Bar et al. 2006; Basso, Dux, Perlovsky, Porte). These processes illuminate how the contents in the conscious field could satisfy the criteria of multiple modules, a form of multiple-constraint satisfaction (Dennett 1991; Merker 2012) yielding the "global best estimate" of what each content should be (Helmholtz 1856/ 1961; Merker 2012). Although these mechanisms explain the underpinnings of good field construction, they do not account for the first-person perspective or subjectivity.

Related to Blackmore, Gur, Mudrik, and Lin's comments about conscious versus unconscious integrations, the latter can occur for perceptual (e.g., intersensory) processing, smooth muscle control, motor programming, and stimulus-response reflexes. Unconscious integrations also occur in the perception of the flavor of food, which involves the combining of information from multiple modalities (including haptic, gustatory, and olfactory; Shepherd 2006), and in pain perception, in which there is, for example, interaction between sensory (lateral pain system) and affective (medial pain system) components (Melzack \& Casey 1968; Nagasako et al. 2003). Evidence reveals that, even for high-level executive processing, much of what transpires is actually unconscious (Dehaene 2014; Suhler \& Churchland 2009), as in the case of determining tendencies (Ach 1905/1951). In response to Mudrik's comment about the conscious penetrability of perception, it is important to reiterate that rivalrous percepts (e.g., the Necker cube or binocular rivalry) or intersensory illusions (e.g., the McGurk effect) are resolved unconsciously: At one moment, consciousness is occupied by only one unambiguous interpretation of the stimulus (Merker 2012). One is conscious only of the outcome of any competitive processes. Thus, for an experimental subject, the McGurk effect ${ }^{2}$ "just happens" in much the same manner as does dream content (Lin, Porte). The only conflicts that one is conscious of are associated with action selection.

We agree with Basso, Franz, Gur, Lin, Mudrik, Pahlavan \& Arouss, and Schwartz \& Pournaghdali that the conscious field affords a type of processing in which the behavioral response to a given content (e.g., a stimulus eliciting aggression or the McGurk effect) is modulated (or "framed") by the other contents (e.g., smoke) composing the field (Fig. R2). Accordingly, as Lin notes, responding adaptively to a complex array of stimuli (e.g., a 
visual scene) requires consciousness. The conscious field permits downstream, motor-related processes to respond to a stimulus in a manner that is contextually sensitive. This kind of sensitivity appears to be unlike anything we find in the robots of today. In short, the conscious field solves the problem, in Behaviorism, of the complex discriminative stimulus.

This sensitivity has led some to conclude that the conscious field is for flexible responding (Basso, Franz, Gur, Lin, Mudrik, Pahlavan \& Arouss, Schwartz \& Pournaghdali). PFT is in accord with proposals in which consciousness affords what appears to be a flexible, multidetermined response (Crick \& Koch 2000; Searle 2000; Sergent \& Dehaene 2004). However, it should be clarified that consciousness, over time, seems to be more flexible than it is. PFT reveals that the same contents will always yield the same voluntary action, with the combination of contents wholly and exclusively determining voluntary action selection. Consider that, if one experienced half of what is normally the whole conscious field, though behavior would certainly suffer, one would not notice the absence of information independent of a content generated by a system dedicated to detecting such discrepancies. In this way, confusion and other forms of metacognition are not givens. (Similarly, the perception of time and spatial "perspective," whether in the first-person perspective or third-person perspective, are not givens [Einstein \& Infeld 1938/1967]. Instead, they are mental creations that are experimentally manipulable; Ehrsson 2007.) These contents are generated by devoted systems that constrain action selection, as Schwartz \& Pournaghdali note. In the conscious field, there is often the absence of information but not information about absence (Bridgeman, personal communication, April, 16, 2014; Simons \& Levin 1997). Accordingly, and related to Hommel \& Wiers's insights about addiction, urges in the field can be short-lived but have, in the absence of strong tendencies against them, a strong influence on behavior (Loewenstein 1996).

Faced with the apparent flexibility of consciousness, one might propose that the function of consciousness is one of instantiating stimulus-response relationships that are "arbitrary." One problem with this hypothesis is that (a) it is difficult to define what constitutes an arbitrary mapping between perception and action; (b) there are countless cases of unconscious processes that seem to involve arbitrary mappings, as in the case of motor programming (Grossberg 1999; Rosenbaum 2002); and (c) some non-arbitrary mappings (e.g., holding one's breath yields a negative subjective state) never become unconscious, despite extensive training and rehearsing of the perception-to-action mappings (Poehlman et al. 2012). Moreover, unlike PFT, this hypothesis fails to explain why smooth muscle actions and intersensory conflicts are always mediated unconsciously.

\section{R3. The focus on simple cases: Low-level versus high-level conscious contents}

To investigate the primary function of consciousness, we were influenced by how progress was achieved in the field of physics (Einstein \& Infeld 1938/1967). Hence, in the target article, we focused on simple cases and lowlevel phenomena (e.g., percepts and urges). Regarding urges, in our "creature in the cave" scenario, the noxious stimulus leading to an inborn avoidance tendency could have been, instead of smoke, some other odorant (e.g., hydrogen sulfide eliciting an inborn disposition of disgust; Gallo, Gur, Lathe, Mudrik). Our point was to illustrate that the inborn action tendency toward the smell changed the manner in which the creature responded to perceptual features of the scene (e.g., the opening) that were already represented in the conscious field. In response to Gur's comments about the capabilities of our creature in the cave, we should emphasize that we interpreted as parsimoniously as possible the operations giving rise to the behavior of this creature, a creature that is not as hypothetical as we thought (see D’Souza \& Bremner).

Regarding the ubiquitous distinction between high- and low-level conscious contents (de Vries \& Ward; Dux; Franz; Gur; Hardcastle et al.; Hommel \& Wiers; Jordan \& Vinson; Melo, Koscik, Vrantsidis, Hathaway, \& Cunningham [Melo et al.]; Mudrik; Perlovsky; Swain, Caluser, Mahmood, Meldrim, \& Morelen [Swain et al.]; Vonasch, Masicampo, \& Baumeister [Vonasch et al.]), one contribution of our action-based approach is that these distinctions are unnecessary. In PFT, all of these different kinds of contents can be construed as tokens which, through the conscious field, constrain voluntary action. From this standpoint, a percept, urge, nausea, and even a high-level sense, such as the sense of agency (which is experimentally manipulable [Riddle et al. 2015] and was touched upon by Gur, Massaro \& Rowe, Mudrik, Pahlavan \& Arouss, Prinz, and Schwartz \& Pournaghdali), are just contents in the conscious field that constrain action selection. For example, while driving, a tip-of-the-tongue state concerning important information could influence action selection (e.g., one decides to park in order to concentrate on retrieval; cf. Schwartz \& Pournaghdali). Hence, a percept, urge, and even a metacognition are similar in terms of their functional consequences. From this standpoint, a primitive form of self is necessary, not for the instantiation of consciousness (as in Prinz 2012; see Prinz's commentary), but rather for adaptive action selection.

In addition, for adaptive action, the highest of conscious contents must be married, in a sense, to the representational format of the lowest and phylogenetically oldest contents: High-level contents must exist in the same decision space and share the representational format of contents as primitive as those of olfaction. Hence, knowledge of the most primitive systems involved in the conscious field will reveal much about the nature of the highest systems, such as those of concern to Dux and Vonasch et al. As de Vries \& Ward note, the latter are constrained by the former, which might be the most tractable to investigate. This is consistent with Shepherd's (2007) conclusion that "the basic architecture of the neural basis of consciousness in mammals, including primates, should be sought in the olfactory system, with adaptations for the other sensory pathways reflecting their relative importance in the different species" (p. 93). In humans, skeletomotor action conflicts often include a medley of high- and low-level contents. ${ }^{3}$ This is evident when, faced with a yellow traffic light, one decides to begin to brake or to speed up (it depends on context; see Woodman \& Vogel 2005, p. 111). It is also evident in Merker's "burnt broccoli" example and in the following scenario: "I am hungry and desire steak, but I am Catholic, and it is Good Friday." Our "Thanksgiving dinner" example was 
Response/Morsella et al.: Homing in on consciousness in the nervous system

intended to demonstrate how a high-level function-language - must take into account contents from a phylogenetically older function - that is, olfaction. Consistent with Melo et al., Schwartz \& Pournaghdali, and Merker, the high-level contents (including metacognitions and emotion) participate with the low-level contents in the constraining of action selection. Accordingly, Freeman (2004) proposes that conscious representations of different sensory origins must at some level be similar in form in order for them to be integrated into a polysensory Gestalt of the world. The format must permit interaction between perceptual and motor systems (Freeman 2004) if there is to be perception-to-action translations (W. Prinz 2003b).

In agreement with Vonasch et al., much can be learned from what has been regarded as high-level contents (e.g., our insights about the McGurk effect and subvocalization, Helmholtz’s [1856/1961] insights about automatic reading). The focus on simpler aspects of human behavior reflects only the presently intended scope of PFT. It is hoped that, after empirical and theoretical developments, the model will generalize to more complex phenomena, including aggression (Pahlavan \& Arouss), taste (Gallo, Lathe), development (D'Souza \& Bremner), emotion (Gainotti, Melo et al., Pahlavan \& Arouss), addiction-related behaviors (Hommel \& Wiers), dream consciousness (Porte), and parental consciousness (Swain et al.). The commentators have illuminated the under-explored action-related aspects of these phenomena. Emotional phenomena, with their actionrelated components (Melo et al.; Frijda 1986), provide a rich domain in which to develop PFT (Gallo, Gainotti, Melo et al., Pahlavan \& Arouss). (As Gainotti notes, affective neuroscience provides additional evidence for a cortical account of consciousness [see also Dux].) Concerning human development (D’Souza \& Bremner, Swain et al.), we were delighted to learn that our creature in the cave may exist not only hypothetically. Such insights from the commentators will help test and extend PFT. In addition, our aim is to eventually unify PFT, already a synthesis, with frameworks concerning ecological perception (de Vries \& Ward), attention (e.g., the allocation of attention to action model of Franz), emotion (e.g., the iterative reprocessing model of Melo et al.), and chaos and complexity (Hardcastle et al.).

Regarding the generation of high-level contents, it is important to reiterate that, when discussing unconscious inference, Helmholtz (1856/1961) was referring not only to the unconscious generation of low-level contents, but also to the generation of high-level contents (e.g., automatic word reading; Augustinova \& Ferrand 2014). In many cases, high-level contents (e.g., subvocalizations) "just happen," as do low-level contents (e.g., nausea; see Porte; see also reflex-like activations of high-level contents in Haidt [2001] and Tetlock [2002]). To investigate how such high-level contents can arise in consciousness unintentionally and in a reflex-like manner, we developed the $r e$ flexive imagery task (RIT; Allen et al. 2013; see review in Bhangal et al. 2016). In this paradigm, the insuppressible conscious contents are from high-level processes, including involuntary object counting (Merrick et al. 2015), subvocalizations, and even the kind of word transformations used in the childhood game of Pig Latin (Cho et al. 2016). The more we learn from the RIT about the generation of high-level contents, the more this generative process resembles that of low-level contents.
From the standpoint of PFT and in response to Massaro \& Rowe, Mudrik, Perlovsky, Schwartz \& Pournaghdali, and Seth, the outputs from a "narratorium" or an "interpreter" module that draws coherent (albeit often incorrect) conclusions about the current context (Roser \& Gazzaniga 2004) are just another kind of content, produced by dedicated systems that participate in the conscious field. Consciousness, which is passive, does not reason or draw conclusions. Instead, systems devoted to reasoning or to the drawing of certain kinds of conclusions (e.g., about a puzzle or a piece of music) have their outputs represented in the conscious field, just as a modular system can maintain an undesired "earworm" in consciousness.

PFT provides a similar answer regarding memory. We agree that many memorial processes are consciously impenetrable (Schwartz \& Pournaghdali) and that the activities of content generators are influenced by past experiences. Memory is essential for the adaptive generation of, and response to, contents (Basso, D'Souza \& Bremner, Gur, Hardcastle et al., Hommel \& Wiers, Jordan \& Vinson, and Lathe). However, these processes stem from dedicated systems operating outside the conscious field, which itself has no memory. (This is evident in neurological conditions.) Similarly, anticipatory processing (Jordan \& Vinson, Seth), which is essential for perception and other forms of content generation, occurs outside the conscious field, which is not burdened with the operations of memory, prospection, or deliberation (Hardcastle et al.). (Relevant to the experimental findings by Hardcastle et al., which involved dissociations between perception and action, expressed actions could sometimes influence, not perception, but only the memory of the expressed action; see Cooper et al. 2012.)

\section{R3.1. Metacognition and consciousness across the phyla}

Compared to all of the functions that have been proposed to be the primary function of conscious processing, the proposed function of consciousness in PFT is the most basic and primitive one. In response to Rosenbaum's point about animal consciousness, PFT, when further developed, might serve as a tractable framework with which to investigate consciousness in animals. However, at this stage of understanding, it is premature to apply our framework to other animals. Across the phyla, it is the case that the same function (e.g., locating an object) can be carried out by vastly different mechanisms (e.g., vision vs. echolocation). Therefore, one cannot conclude that a given action (e.g., holding of breath) in species $X$ is carried out by the same mechanisms by which it is carried out in species $Y$. Even within one species, the same function could be carried out by more than one mechanism (Dawkins 1982). Hence, before making cross-species comparisons, which are problematic, science must explain the perplexing contrasts within self-reporting, adult humans. (This conclusion is relevant also to the idea of extending PFT to infants; D’Souza \& Bremner.) At present, in humans alone there are sufficient contrasts (e.g., the pupillary reflex vs. conscious pain) that are difficult to explain. (In addition, as Swain et al. note, the most basic of human functions - as simple and primitive as they have been deemed to benonetheless seem to occupy much of the brain's activity and to dominate much of human existence, especially during the most critical of periods [e.g., early childhood, parenting].) 
Response/Morsella et al.: Homing in on consciousness in the nervous system

Nevertheless, it is worth reiterating that, because our approach is simplistic and evolutionary-based, it may be the most suitable framework to be extended to the study of other animals. Insofar as one would like to study consciousness across species, one should focus on olfaction, for several reasons, including that it is the most phylogenetically preserved modality and a "common denominator" region of the vertebrate brain. Moreover, when conducting experiments on adult humans, it is much easier to induce olfactory percepts than high-level, metacognitive states and background states, such as those mentioned by Basso, de Vries \& Ward, Franz, Perlovsky, and Schwartz \& Pournaghdali. For instance, regarding tip-of-the-tongue states and feelings of knowing, it is difficult to control exactly when these states arise, and little is known about their neural correlates. At this stage of understanding, it is progressive to focus on obvious, easily reportable forms of conscious content (e.g., detecting a smell) and not on more nebulous conscious states (e.g., background states). The lack of verbal report regarding the stimulation of frontal areas may indeed reflect the greater difficulty of communicating about these contents than about perceptual events. Hence, in the current version of PFT, we limit ourselves to the kinds of conscious contents occurring in our "creature in the cave" scenario. Nevertheless, these high-level states, which involve the frontal cortex, may provide unique insights regarding consciousness.

Franz questions whether olfaction is a model system for consciousness research. Justifications for our focus on olfaction can be found in the target article (see Note 1 and sect. 3.5). We are grateful for Merker's listing of additional properties of olfaction (e.g., minimal spatialization, habituability of ordinary odorants, innate preferences, pheromonal signaling) that render it a good modality for investigating consciousness. As Gallo notes, other unique properties of olfaction are that there is no such thing as a "neutral" odorant and that swallowing is almost always a voluntary act. Concerning habituation (Merker), this phenomenon occurs in a special manner for olfaction because of the absence of the possibility of voluntary re-access to an exposed odorant (Merker; see also Stevenson 2009). Regarding the "experiential nothingness" associated with habituation, research indicates that (a) activation in the orbitofrontal cortex does not decrease over odorant exposure (60 seconds; Poellinger et al. 2001), and (b) accurate odor detection persists after activation in the piriform cortex decreases to a baseline (or below baseline) level (Poellinger et al. 2001; Sobel et al. 2000). When isolating the neural correlates of olfactory consciousness, one should seek regions that are active most during conscious detection but not during habituation or subliminal perception (Merrick et al. 2014). Merker's forward-looking insights also reveal that much can be learned about olfactory consciousness by examining the activities of cortical layer VI pyramidal cells and the effects on olfactory consciousness from perturbations (e.g., lesions) of the dorsal pulvinar, a multimodal region whose activity is tightly linked with conscious perception (Wilke et al. 2009).

\section{R4. Passive frame theory explains the primary function of consciousness}

Prinz astutely questions whether PFT provides a functional account of consciousness. (Prinz also requested a definition of subjective experience that is distinct from our functional account of consciousness. This definition is provided in section R1.) We agree that a constellation of isolated facts (e.g., that skeletal muscle but not smooth muscle can be consciously controlled) does not by itself constitute an explanatory model, just as one can know that the sun rises every morning and that the seasons change without having an explanatory model of the solar system. Thus, it is one thing to know that skeletal muscle can be consciously controlled, but it is an entirely different matter to have an explanatory framework that integrates such a fact into a coherent, causal account. PFT provides such a framework.

Perhaps this issue is better illustrated by analogy. In naively observing the digestive system, one could generate hypotheses concerning the circumstances under which, say, salivary amylase is secreted into the mouth. However, these hypotheses would be fundamentally different from those regarding the primary function of salivary amylase, which would have to propose, in addition, what it is for (e.g., digestion of starch in the mouth) and, by extension, what phenomena would occur to a lesser degree without it. As a theory about function, PFT supersedes these criteria by claiming that (a) consciousness is necessary for collective influence over the skeletomotor output system, (b) no other process performs this role, and, (c) without these states, collective influence and integrated actions would be absent. Except for the fact that the actions of salivary amylase upon starch can be observed directly (e.g., in a Petri dish), the functional claims about this enzyme and consciousness are analogous.

Then, of course, there are questions regarding how salivary amylase breaks down starches and why salivary amylase, and not some other substance, was selected in evolution to carry out this function. With respect to biological systems, how and why questions are fundamentally different from what for questions (Lorenz 1963; Simpson 1949). PFT explains what consciousness is for, that is, the nature of its primary function. How physical processes carry out collective influence is a variant of the "hard problem" and is thus outside the scope of PFT. In addition, one must distinguish the primary role of evolutionary adaptations from their secondary roles (Dawkins 1982; Gould 1977; Lorenz 1963). A scientist could argue, for example, that color perception evolved for selecting fruits and detecting camouflaged prey, but no one doubts that color perception could also be used to appreciate a painting. The color harmony of a painting is perceptible to us because it involves the kinds of stimuli that are of adaptive significance in other contexts. In response to Prinz, we perceive the kinds of things that we evolved to act upon (Dawkins 1982; LeDoux 2012). As humans, we have inherited the conscious field. Like the eye, it has a fixed architecture, one that, though having a net adaptive effect across all of the stages of ontogeny, may not function adaptively in all contexts. Its adaptive value may not be evident in one particular situation (e.g., when experiencing unhealthy urges).

\section{R4.1. The conscious field does not require conflict or skeletal muscle}

In response to comments about the liaison between conflict and consciousness (D'Souza \& Bremner, de Vries \& Ward, Gur, Hardcastle et al., Hommel \& Wiers, 
Lathe, and Massaro \& Rowe), in PFT, a content does not require conflict to enter the conscious field, which is a "continuous feed" system. The conscious field is not for conflict monitoring per se, but rather for collective influence, which is especially important under conditions of conflict. (We agree with Jordan \& Vinson that people underestimate how many action conflicts occur on a given day.) In PFT, contents, because of the requirement of encapsulation, do not know about the nature of other contents nor about whether there is conflict. In addition, the contents do not know whether they are relevant for ongoing action. The notion that no conscious content can directly influence another conscious content, either at the same time or across time, is consistent with Gestalt theory (e.g., Werner and Kaplan's [1963] notion of dynamic schematization) and with Helmholtz's (1856/1961) "global perception," in which unconscious configurations of afference interact to generate an unambiguous conscious field, which can be construed as a static mosaic of conscious contents. As Merker (2013c) notes, the kinds of sensorial interactions involved in illusions (the McGurk effect), color constancy phenomena, and other perceptual phenomena occur, not in the conscious field, but in the (unconscious) processing of afference, occurring before the construction of the conscious field. For instance, though a content such as the McGurk effect is influenced by different kinds of configurations of afference, once the content is conscious, it cannot be modified on the basis of other contents. In PFT, the contents are not there to communicate with each other.

Concerning Rosenbaum's question about animals lacking skeletal muscle, in PFT, there is nothing intrinsically special about skeletal muscle that causes it to be related to consciousness. Skeletal muscle is one of many "multidetermined" effectors in the body. (Consider that the pupillary reflex, involving smooth muscle, too, is multidetermined, as it is influenced by light conditions, emotions, and other variables.) Conscious processes are distinguished from unconscious ones not simply because they "involve" skeletal muscle, but because they involve skeletal muscle in a particular manner, in which encapsulated systems (having different operating principles and phylogenetic origins) vie to express their respective skeletomotor plans.

Pertinent to Rosenbaum's question, one can also ask: If conscious states are primarily for skeletomotor action, then why do they persist even when the skeletomotor system is deactivated because of, for example, neural damage? In response to this criticism, one should consider the following analogy. Many of today's automobiles contain navigational systems whose primary function is to aid navigation. With this in mind, it is conceivable that the navigational system would continue to function despite problems with, say, the transmission of the car. Similarly, conscious processes, whose primary function is serving skeletomotor action, can continue to function after the peripheral structures that they are intended to serve are nonoperational. (Similar decoupling of central conscious processing from peripheral events occurs in phantom limb; Ramachandran 1999.)

\section{R5. Attention, automaticity, and the timescale of consciousness}

In response to the concerns of Basso, Dux, Lin, and Franz about the relationship between attention and consciousness (see differing views about this relationship in Koch and Tsuchiya [2007] and Cohen et al. [2012]), we posit that the nature of this relationship depends in large part on one's definition of attention. As noted by Tsotsos (2011), there are more than a handful of definitions of attention. Most theorists construe attention as a cause, something that influences information processing in a certain way, while other theorists, interestingly, construe it as an effect, for example, as a by-product of a valuebased selection process centered on the basal ganglia (Krauzlis et al. 2014). In addition, for Oberauer and Hein (2012), there is a low-level form of attention, having certain properties, and a separate, higher-level form of attention, having other properties. It could be argued that one of these forms of attention, but not the other, is somehow necessary for basic consciousness. From our standpoint, it has to be explained how attention, defined one way or another, is necessary for the detection of basic conscious contents such as nausea or a gas leak (see related Merker comment about olfactory attention).

We next turn to the topic of automaticity, which was raised by Gur, Massaro \& Rowe, and Rosenbaum. Regarding the contrast between novel actions (e.g., the first time one ties one's shoes) and automatized actions (e.g., the ten thousandth time one ties one's shoes), it is difficult to ascertain which aspects of consciousness, if any, are diminished in the latter. In making such a contrast, one could easily conflate changes in consciousness and the well-known changes in attentional processes that stem from automaticity (Baars 1997b; Logan et al. 1999; Puttemans et al. 2005). The question is: What would be no longer consciously accessible when driving home automatically? One benefit of PFT is that when contrasting conscious and unconscious processes, the contrast is between processes that one is never conscious of (e.g., the pupillary reflex and peristalsis) and processes that one is almost always conscious of (e.g., pain and air hunger while holding one's breath).

In response to Hommel \& Wiers's thoughtful question about the timescale at which consciousness operates (related to Massaro \& Rowe's comments), we should state that according to PFT, the timescale must be that associated with normal, ongoing voluntary action selection, which is different for different kinds of voluntary acts (e.g., taking a deep breath vs. looking left to right) and is often slower than that of reflexive actions. Whatever the exact timescale in humans (or in other species; de Vries \& Ward) may be, it must be slow enough for the frame check to include the relevant contents for the adaptive employment of a given effector and quick enough (in the order of hundreds of milliseconds) to benefit actions occurring at fast rates (e.g., voluntary saccades). Regarding the former, it is interesting to consider that the instruction to a subject to press a button whenever there is a noticeable change in any part of the sensorium requires the action processes associated with that effector to, in a sense, sample a wide variety of contents in a very short time.

\section{R6. Evolution, functionalism, and emotion}

Prinz and others (Massaro \& Rowe, Mudrik) question why the function attributed to consciousness is not solved unconsciously, as are many other functions. After all, it is 
easy to imagine integrated actions (e.g., suppressing inhalation) occurring without anything like a conscious field. However, there are many hypothetical solutions to phylogenetic challenges that the human body did not arrive at by way of evolution. In our descriptive account, intuitions regarding how the nervous system should work take a back seat to actual data revealing the manner in which it actually works. Hence, it seems premature to adopt an epiphenomenal stance (e.g., Blackmore) until there is a sufficient scientific understanding about the place of consciousness in nature. We should add two comments. First, our intuitions of how nervous systems should function, in which sensory inputs are connected to motor outputs, are actually computationally impossible (Tsotsos 1995; 2011). (Regarding this issue, Neal Miller [1959] hypothesized that "central states" could provide a solution to the problem; see discussion in Corr \& Morsella 2015.) Second, some of the counterintuitive adaptations in evolutionary history (e.g., intra-psychic conflict) are actually good solutions given the hardware at hand (e.g., slow processing units; Livnat \& Pippenger 2006).

One could also ask: If consciousness is for action selection, then why is one aware of so many things that do not demand immediate action (e.g., the plot of a story; Basso, de Vries \& Ward, Gur, Perlovsky, Porte)? One could certainly imagine more efficient systems - and more falsifiable models - that invoke the conscious field only under conditions when it is needed most (e.g., conflicting action tendencies). The apparent inefficiency of the field is an incontrovertible, first-person property of these states. In the absence of any conflict or obvious demands upon action, one is continuously conscious of, say, a red object standing before one. This counterintuitive property of the field can be likened to the efficiency of the continuously running conveyor belt of the ball-return machine at a bowling alley (Morsella 2005), which is inefficient in the sense that it constantly expends energy, even when there are no bowling balls needing to be returned to players. However, the machine is more efficient than a machine having an additional mechanism that determines whether a ball needs to be returned. Such deceptively "inefficient" solutions can be observed in physiology outside the nervous system, as in biological filters (e.g., the kidneys), which continuously filter a substrate regardless of the status of the substrate. Just as eyes do not turn off when there is nothing interesting to look at, the conscious field does not turn off when its role is unneeded.

Regarding one's consciousness of a story that may never influence behavior, it should be stated that simulacra such as novels have been constructed to incite attentional, emotional, and other kinds of processes for only an infinitesimally recent fraction of human history. Consistent with PFT, the stimuli from simulacra succeed in part because they activate inflexible, encapsulated systems that, at some level, are incapable of "knowing" that what is occurring is not "real." (Consistent with this view is the idea that emotional systems [e.g., for fear and aggression] evolved independently and are modularized in the brain [LeDoux 2000; 2012; Öhman \& Mineka 2001].) Scary movies, for example, are capable of activating to some degree the kinds of affect associated with the natural observations of the portrayed events. For the majority of our natural history, such activation was clearly adaptive. Though such inclinations and imagery operate in a realm shielded from that of expressed action, they are still intimately related to action. (Relevant to Porte, in certain neurological conditions, sleep paralysis fails and patients act out their dreams, revealing the intimate link between conscious content and action. Sleep paralysis is mediated by inhibition only at the latest stages of motor control [e.g., at the spinal level; Glenn \& Dement 1981].) Thorndike (1905) concludes, "The function of thoughts and feelings is to influence actions ... Thought aims at knowledge, but with the final aim of using the knowledge to guide action" (p. 111).

Prinz asks how the design of consciousness, a product of evolution, reflects the needs of adaptive action control. First, we subscribe to the uncommon position that consciousness is best understood by examining the requirements of adaptive efferent action control rather than the needs of perceptual analysis. ${ }^{4}$ Accordingly, as noted by Merker (2013c), the conscious field is organized egocentrically (see J. Prinz 2007), around an active agent that reaches, locomotes, and performs other acts that are spatially directed. This organization is found also in the dream world (Porte). (PFT pertains to normal waking consciousness, but as noted by Porte, the kind of action selection occurring in dreams seems to be isomorphic to that of waking.) For action selection, it would be disadvantageous for this first-person arrangement to break down and for an object on the left to be represented as if on the right. According to PFT, the first-person perspective and other properties of the field (e.g., its having varied elements) result from the demands of adaptive action selection. Second, the valence and other properties of a conscious content are in some ways isomorphic to ongoing action. It is not the case, for example, that pleasant states are associated with avoidant behaviors or that unpleasant ones are associated with approach behaviors. (As noted by James [1890], this non-arbitrary relationship between valence and action poses a problem for epiphenomenalism.) Similarly, as Sperry (1952) notes, and consistent with de Vries \& Ward's discussion of affordances, perceptual contents (e.g., the shape of a triangle) are often more isomorphic with action plans (e.g., tracing) than with sensory inputs (the proximal stimulus on the retina). In conclusion, much of the way the field is designed, including a primitive sense of self (the first-person perspective), reflects the demands of adaptive action selection.

\section{R7. Integrated ("voluntary") action and subliminal stimuli}

The evidence for PFT is the combined evidence for each of the hypotheses composing the framework. For such a synthesis, which attempts to account for so many disparate observations, there will naturally be both evidence for and against each of its constituent hypotheses. For example, there are bits of empirical evidence that, at first glance, appear to challenge the hypotheses of encapsulation and ideomotor processing and that consciousness is dependent on cortical processes. In the coming years, the evidence for and against the tenets composing PFT should be examined carefully. Some of the tenets of PFT will be amended; others will be abandoned.

Nevertheless, today there is substantially more evidence in favor of PFT than there is against it. Moreover, much of 
Response/Morsella et al.: Homing in on consciousness in the nervous system

the evidence supporting PFT can be obtained, not just in the laboratory, but in everyday scenarios (e.g., the unawareness of peristalsis and the pupillary reflex vs. the subjective experience of holding one's breath). These bits of evidence, realized in everyday life scenarios and not dependent on the technicalities of the laboratory, are often the most compelling forms of evidence. PFT focuses on modal findings (i.e., the most common findings) regarding when conflicts are conscious and when they are not. At this stage of the scientific understanding of a problem as thorny as consciousness in the brain, the mode is very informative.

Ayars mentions the well-known finding in which a prime (e.g., a rightward arrow) is presented subliminally before a target stimulus (e.g., a leftward arrow) to which the subject must respond. In this paradigm, the response to the target is modulated by the prior presentation of the prime. For example, interference (e.g., increased response latencies) is less when the two arrows match (the congruent condition) than when they mismatch (the incongruent condition; Eimer \& Schlaghecken 2003; Schlaghecken et al. 2006). Similarly, the subliminal presentation of the image of a spider could, in principle, ramp down an appetitive, behavioral "approach" system. The data mentioned by Ayars are corroborated and complemented by many similar findings (e.g., Hughes et al. 2009; Van Opstal et al. 2010), some of which stem from our own laboratory, in which subliminal stimuli induced behavioral inclinations that induced subjective urges and also influenced behavior, even though subjects were unaware of the source of these urges (discussed further on here and in Morsella et al. 2011). Accordingly, Desender et al. (2014), after reviewing the literature on subliminally induced inhibition in interference paradigms, conclude, "The difference between awareness of a prime and experience of a conflict is of crucial importance ... Response conflict might give participants the general feeling that something is wrong, without their knowing why or what is wrong" (p. 681). To Desender et al. (2014), this subjective experience of conflict, regardless of awareness of the prime, is essential for the top-down control of behavior.

The effect described by Ayars could arise from various mechanisms (Logan et al. 2015; Munakata et al. 2011), including negative priming (Tipper 1985) or the residual effects from the prime having activated, through unconscious efference binding, a response code (consistent with section 3.2 of the target article) that does not match the subsequently activated response code. (In section 3.2, we also discuss the relevant fact that motor programs, which are unconscious, are modulated in sophisticated ways by external stimuli.) We agree that past experience can influence the activities of content generators and response systems (discussed subsequently). For example, a response code is likely to function more quickly when it was activated recently (repetition priming) than when it was not activated recently.

Second, and more important, we should clarify that collective influence from a frame check pertains to a class of phenomena (e.g., holding one's breath while underwater or suppressing some other, prepotent and simultaneously activated action plan) that is fundamentally different from the kind of established, sequential priming effect described by Ayars, in which a prime modulates (but does not fully suppress) a subsequent behavior. As noted in Morsella and Bargh (2011):
The level of activation of the plans involved in integrated action is far beyond that of "sub-threshold" activations. For example, in psycholinguistic research, there is substantial evidence that naming "dog" primes the action plan for naming a member of the same category (e.g., "horse"; Levelt, 1989). The level of activation that we are speaking of in our definition of integrated action is far above this threshold -it is at the level of activation at which action plans would not only influence overt action but also trigger action. (p. 341)

They also state that "integrated action occurs when two (or more) action plans that could normally influence behavior on their own (when existing at that level of activation) are simultaneously co-activated and trying to influence the same skeletal muscle effector" (Morsella \& Bargh 2011, p. 341). These actions occur when one holds one's breath, refrains from dropping a hot dish, suppresses the urge to scratch an itch, or makes oneself breathe faster for some reward (Morsella 2005; Morsella et al. 2009a). These are discrete, goal-directed actions (e.g., carrying an object, depressing a lever), behaviors Skinner (1953) characterized as operants (pp. 14-15). Based on ideomotor theory (e.g., Harleß 1861; James 1890), PFT proposes that the conscious contents associated with behavioral control are action outcomes in the world (e.g., a button depressed) or the body (e.g., fingers snapping). This addresses the second question by Rosenbaum.

Before returning to the experimental effect mentioned by Ayars, we should add that, through collective influence, the field permits for a massive many-to-one (or, at least, many-to-few) conversion (Merker 2013c), for there are many action-related contents but only a few operants that, at one time, direct behavior. This may reflect the mechanical limitations of the skeletomotor system, in which only one word can be uttered at a time (Wundt 1900), or, more precisely, in which only one (or a few) operant(s) can be expressed at one time. As noted by Rosenbaum, this aspect of PFT should eventually be integrated with research on the psychological refractory period (Pashler 1993; Welford 1952), which, though not directly concerning conscious processing, yields conclusions that are consonant with PFT. As Pashler (1993) concludes:

The limitations in carrying out stimulus-response tasks concurrently are not introduced at the level of stimulus perception, nor in production of the motor response. Those mental operations can work in parallel. Rather, the problem is in deciding what the response will be, and this kind of mental operation seems to be carried out in series - that is, one task at a time. (p. 52)

One of our aims is to first integrate PFT with this important research (along with research on the role of action control on attention; e.g., Allport 1989; Neumann 1987) and then isolate, within the architecture of PFT, the locus of the action-selection bottleneck. For now, PFT is consistent with Jackendoffs (1990) view that consciousness reflects some form of intermediate, action-planning stage inbetween sensory and motor processing. More specifically, we propose that consciousness is associated with stages that, though clearly subsequent to those of sensory processing (Hochberg 1998; Logothetis \& Schall 1989; Marcel 1993), precede those of action selection. It seems that one is unaware of the computational products of action conflicts, resolutions that, should they exist (see Kaufman et al. 2015), determine the general course of observed action (pressing one button instead of another). 
Consciousness reflects action options, both those that are selected and unselected.

The co-activation of action plans in integrated action can be indexed by behavioral and neural measures, as there are several behavioral and neural features that could be used to distinguish integrated from unintegrated action. For example, like any behavior of low strength, conflicted action (a form of integrated action) is easier to perturb than un-conflicted or unintegrated action (Skinner 1953). Unlike the conscious field, overt behavior is "integrated" in the sense that only one discrete operant is manifest (Lin), even though behavior is influenced by two action plans, as in the case of the Stroop incongruent condition. Unlike in the experimental effect described by Ayars, collective influence permits for one operant to be expressed, leading to only one effect in the world (e.g., a button pressed), while another operant is (almost) fully suppressed, leading to no noticeable effects in the world..$^{5}$ Interestingly, the suppressed operant in a conflict could be the prepotent plan, as in the case of holding one's breath. (Investigators have begun to examine the behavioral consequences of such unselected plans; Filevich \& Haggard 2013.) With this in mind, we can respond to Jordan \& Vinson's insightful point challenging the distinction between perception and action. First, in natural selection, it is overt behavior and not musings and mentations that are directly selected. The latter, along with mental simulations, are, in a sense, less costly than is overt behavior. Importantly, behavioral inclinations can often be behaviorally suppressed but not mentally suppressed (Bargh \& Morsella 2008). Second, the distinction between perception and action is an informative one because motor control, unlike perception, is largely unconsciously mediated. This is one of the many differences between perception and action.

Relevant to the critiques by Blackmore, Lin, Mudrik, and Prinz about the explanatory power of PFT, the framework reveals that, unlike involuntary actions (e.g., dropping a hot dish because of the pain-withdrawal reflex), voluntary actions can be construed as a form of integrated action. Hence, PFT defines voluntary action in ways more informative than the common "homuncular" definition of these acts - that an action is voluntary if the organism intended to do it. As noted by Passingham (1995), voluntary actions are special in that they can be suppressed; from the present standpoint, the act of suppression (suppressing a cough) is an archetypal integrated action. Again, this act is different from many forms of inhibition in the nervous system, many of which are unconscious, as in the case of lateral inhibition and negative priming. (Relevant to Ayars, PFT does not state that inhibition requires consciousness.) One might then argue that, instead of proposing a framework such as PFT, it is more parsimonious to hypothesize that the role of consciousness is to suppress actions, for holding one's breath or performing response interference tasks (e.g., the Stroop task) involves response suppression. However, this fails to account for the role of consciousness in integrated actions such as breathing faster for some reward, which requires collective influence but not suppression.

Regarding subliminal stimuli, we agree that these controversial stimuli can influence subsequent behavior in one way or another (see review in Morsella \& Bargh 2011). (Consistent with the conclusions of Lin, some have argued that subjects do perceive these stimuli but that, for some reason [e.g., confabulation or distortions of memory], subjects fail to report about the conscious percept [Block 2007].) Moreover, unlike many, we do believe that these controversial stimuli can be regarded as unconscious. Interestingly, in some cases, the subject can be unconscious of the stimulus but be aware of the skeletomotor urges engendered by them (Morsella et al. 2011), which is consistent with the idea that one can be aware of skeletomotor inclinations (e.g., urges) but be unaware of the sources of these inclinations, as in many of the cases mentioned by Lathe and Seth and as found in research from our laboratory (Morsella et al. 2011) and in the classic research by Nisbett and Wilson (1977), in which participants were unaware of the factors influencing their decisions (e.g., to aid a stranger). Thus, people can be conscious of tendencies (e.g., urges and cravings), but not necessarily of the factors engendering the tendencies (Baker et al. 2004; Nisbett \& Wilson 1977). For example, the subjects in Nisbett and Wilson's experiments were certainly aware of their "urge" to, say, help a stranger who had collapsed. If the subjects had been physically unable to aid the stranger because the stranger was in a precarious environment, then subjects would have certainly reported that, though they suppressed helping behavior, they nonetheless experienced the urge to help. This inclination would be conscious even though the factors giving rise to it would be unconscious. PFT predicts that the operating principles within content generators (e.g., for urges) can be opaque to awareness, as in the unconscious factors that engender addiction-related urges (Baker et al. 2004). Accordingly, research has shown that people can have inexplicable "gut feelings" (or "somatic markers"; cf. Damasio et al. 1991) reflecting the response tendencies of systems whose inner workings and learning histories are opaque to awareness (LeDoux 2000; Öhman \& Mineka 2001; Olsson \& Phelps 2004). In short, the source of a response tendency is distinct from the awareness of that inclination.

Thus, in the kind of experiment mentioned by Ayars, subjects might be unaware of the stimuli but be aware of the skeletomotor urges triggered by such stimuli. Desender et al. (2014) conclude that such awareness is required to counteract the interference effects from response conflict induced by subliminal or supraliminal stimuli. We have found such effects with subliminal stimuli (discussed in Morsella et al. 2011) but have found it challenging to prove unequivocally that the stimuli were unconscious (cf. Lin). In the kind of experiment described by Ayars, one must examine not only whether subjects are unaware of the stimuli and, should they exist, the stimulus-elicited urges, but also that the contrast between the two conditions is not driven solely by a facilitatory effect from the congruent condition.

We should add that subliminal stimuli are problematic also because they are stimuli of very weak strength, unlike the kind of stimuli on which unconscious processes usually operate (Bargh \& Morsella 2008). Hence, in several studies (e.g., Molapour et al. 2011; Morsella et al. 2009a; 2009c), we induced action-related urges by the presentation of supraliminal distractor stimuli in paradigms such as the Stroop and flanker tasks. Consistent with Nisbett and Wilson (1977), it is unclear whether subjects were aware of the source of these urges (see discussion in Morsella et al. 2009c). For example, though the urges 
Response/Morsella et al.: Homing in on consciousness in the nervous system

arising from different flanker conditions were systematic (Morsella et al. 2009c), it seemed that subjects were unaware of why urges differed across conditions. To further investigate how action-related conscious contents can be triggered systematically and unintentionally by supraliminal stimuli, we developed the aforementioned RIT. With this paradigm, one can examine how unconscious processes operate over supraliminal stimuli. According to the traditions of Freud and Helmholtz, this is the usual way in which unconscious processes operate.

The conclusions on which PFT is based do not stem from controversial techniques such as visual masking (see Lin). Again, much of the evidence supporting PFT can be obtained in everyday scenarios. Regarding laboratory data, of the many conditions in interference paradigms, the strongest perturbations in consciousness (e.g., urges to err) are found in conditions involving the activation of incompatible skeletomotor plans (Morsella et al. 2009a; $2009 \mathrm{c}$ ), such as in the incongruent Stroop condition or the response interference (versus perceptual interference) condition of the flanker task (see quantitative review of evidence in Morsella et al. [2011]). Conversely, when distinct processes lead to harmonious action plans, as when a congruent Stroop stimulus activates harmonious word-reading and color-naming plans (e.g., BLUE in blue font), there are little such perturbations in consciousness, and participants may even be unaware that more than one plan influenced overt action (e.g., uttering "blue"). This phenomenon, called synchrony blindness (Molapour et al. 2011), is perhaps more striking in the congruent ("pro-saccade") condition of the anti-saccade task (Hallett 1978), in which distinct brain regions/processes indicate that the eyes should move in the same direction (cf. Morsella et al. 2012). Regarding the Stroop congruent condition, MacLeod and MacDonald (2000), after carefully reviewing the behavioral and psychophysiological data, conclude that, "The experimenter (perhaps the participant as well) cannot discriminate which dimension gave rise to the response on a given congruent trial" (p. 386). Last, as mentioned in Note 8 of the target article, experiments have revealed that, in simpler tasks, incompatible skeletomotor intentions (e.g., to point right and left) do produce systematic intrusions into consciousness, but, as predicted by PFT, no such changes accompany smooth muscle conflicts or conflicts occurring at perceptual stages of processing (e.g., intersensory processing; Morsella et al. 2011).

We are in agreement with Bridgeman that there are many perceptual events that are unconscious, as in the case of backward masking, saccadic suppression, change blindness, and changes in self-generated action that are below the just noticeable difference for proprioception (Jeannerod 2006). (The eye, discussed by Bridgeman, is interesting because it includes all of the kinds of actions contrasted in PFT: smooth muscle action [for pupillary reflex], involuntary skeletomotor action [e.g., a blink], and voluntary skeletomotor action [e.g., a wink].) PFT proposes not that all perceptual processes are conscious, but that motor control is unconscious and that, for the little that is conscious in the perception-to-action cycle, it is associated with the former. In short, one is trapped in the sensorium, but, even within it, one is not conscious of everything. Consistent with PFT, that which one is conscious of regarding eye movements consists of the kinds of things that are important for adaptive action selection. Seeing the world as unstable or the blurring of the retinal image would not serve this end.

According to Ayars, D’Souza \& Bremner, Keller, and Schwartz \& Pournaghdali, PFT is too restrictive in proposing that the integrative role of consciousness is only for the skeletomotor output system. (For example, Seth astutely recommends that we extend PFT to include effects upon the autonomic nervous system.) We believe that, first, our restriction renders the framework more falsifiable and fecund, and, second, the majority of the strongest bits of evidence corroborates it. Figuratively speaking, and in response to Lin, people tend not to experience conflictrelated perturbations in consciousness while experiencing the McGurk effect, ventriloquist effect, or conflict in the pupillary reflex (Morsella et al. 2009a), but such is not the case while people perform the Stroop task or exert self-control (Baumeister \& Vohs 2004; Preston \& Wegner 2009), all of which involve skeletomotor conflict. Regarding the related, important insights by Keller, PFT is consistent with the notion that something represented in the conscious field may incidentally, because of the unconscious afference with which the content is necessarily coupled (e.g., the sensory inputs in the McGurk effect), be linked with effects other than those upon skeletomotor action. For example, in indirect cognitive control, which is germane to the sailing boat analogy by Hommel \& Wiers, adult humans often rely on such effects. A process such as salivation, noted by Keller, could be controlled voluntarily only in such a sophisticated manner. Consistent with PFT, because conflicts involving salivation (or the pupillary reflex) do not involve the skeletomotor output system, one is oblivious about their existence.

Regarding the scope of PFT, it is worth noting that, regarding the role of consciousness, PFT is less restrictive than Godwin et al. (2013), who propose that consciousness is a tool used not by all sub-systems in the skeletomotor output system, but by only what has been construed as the instrumental response system (Bindra 1974; 1978; Morsella 2005; Tolman 1948). Moreover, PFT does not go as far as Morsella (2005) in limiting the abilities of unconscious processes (e.g., in limiting the amount of unconscious "cross talk" between systems). However, PFT portrays consciousness as more passive than what is proposed in Morsella (2005).

\section{R8. Clues from PFT regarding the neural correlates of consciousness}

Even though PFT is not about the neural mechanisms giving rise to consciousness, which is Marr's implementation level of analysis, the framework provides new clues regarding the matter and does rule out certain possibilities (e.g., panpsychism). Hence, though PFT does not attempt to solve the hard problem, it does restrict potential candidate explanations.

One benefit of the architecture outlined in PFT is that, for it, the puzzle of the mind-body problem is the same whether (a) the tokens (conscious contents) differ from each other qualitatively or quantitatively, (b) there is one or many tokens (the former might be more theoretically tractable), or (c) the field is unitary or componential. The first is important because we know that the brain can implement quantitative codes. For example, it has been proposed that 
consciousness depends on "precise synchronization of oscillatory neuronal responses in the high frequency range (beta, gamma)" (Singer 2011, p. 43). Uhlhaas et al. (2009) specify that the earliest signature of conscious processing is "the precise phase locking across a widely distributed cortical network" (p. 11). Singer (2011) adds that "brain states compatible with conscious processing should be characterized by a high degree of synchrony" (p. 43). Similar conclusions about the role of high frequencies (e.g., $>30 \mathrm{~Hz}$ ) in consciousness can be found in other projects (Aru \& Bachmann 2009; Crick \& Koch 1990; Doesburg et al. 2005; 2009; Engel \& Singer 2001; Hameroff 2010; Jung-Beeman et al. 2004; Meador et al. 2002; Panagiotaropoulos et al. 2012; Uhlhass et al. 2009; Wessel et al. 2012). More generally, it has been proposed that, to instantiate consciousness of any kind, the mode of interaction among regions (interregional synchrony) is as important as the nature and loci of the regions activated (Buzsáki 2006; Fries 2005; Hummel \& Gerloff 2005; Lewis et al. 2012; Ward 2003).

There is less consensus regarding how, to instantiate the conscious field, high-frequency bands such as gamma (gamma in the rat, ranging from $40 \mathrm{~Hz}$ to $100 \mathrm{~Hz}$; Adrian 1942; Kay \& Beshel 2010) must interact with ongoing, lower-frequency bands. It appears that these interactions between frequency bands are complex and dynamic. In addition, controversy continues regarding which brain regions are primarily responsible for the high-frequency brain rhythms linked to consciousness and whether cortical electroencephalography reflects consciousness (Merker 2012; 2013b).

Olfaction provides a portal for understanding the neural correlates of "additions" to the conscious field. (Olfaction was one of the first systems in which the nature of oscillatory activity in the brain was investigated [e.g., Adrian 1942].) Olfactory information may be encoded through oscillating neural assemblies (Adrian 1942; 1950a; 1950b; Eeckman \& Freeman 1990; Freeman 1975; Kim et al. 2006; Laurent \& Davidowitz 1994). Different odorants elicit different patterns across spatially distributed neural ensembles of the olfactory bulb (Freeman 1987; Laurent \& Davidowitz 1994; Xu et al. 2000).

In our "creature in the cave" example, the smell of smoke is an addition to the conscious field that influences skeletomotor responses toward other conscious contents (e.g., the percept of the opening). Examining the neural correlates of such an addition reveals more evidence for the integration consensus. ${ }^{6}$ In olfaction, it is frequencies in the beta range $(\sim 15-$ $30 \mathrm{~Hz}$ in the rat; Kay \& Beshel 2010; Kay et al. 2009) that link olfactory processing to non-sensory, cognitive areas (Vanderwolf \& Zibrowski 2001; Zibrowski \& Vanderwolf 1997). Specifically, beta oscillations in the olfactory bulb "entrain" both areas of the piriform cortex, suggesting that beta oscillations may serve the purpose of transmitting olfactory information from the olfactory bulb to higher-order, more cognitive areas, including cortical and subcortical areas. Consistent with this view, research outside of olfaction has found that beta may be involved in large-scale coupling for sensorimotor integration (Freeman 2007; Siegel et al. 2012).

In addition, Kay et al. (2009) propose that "beta oscillations are associated with motor models, favoring this oscillation as a good substrate for long-distance communication" (p. 7). Accordingly, beta coherence between the olfactory bulb and the hippocampus accompanies odor learning in a go/no-go task (Martin et al. 2007). (See Lathe's treatment about olfaction and the hippocampus.) It has been proposed that, though the higher frequency of gamma (in the rat, 40-100 Hz; Kay \& Beshel 2010) can be observed in processing at primary sensory areas, when the sensory information becomes part of a wider network which includes activations from other sensory modalities, then the frequencies are in the beta range (Freeman 2007). (Mechanisms engendering gamma during odor perception reside within the olfactory bulb; Freeman 1979.) Based in part on such neural evidence, it has been hypothesized that one becomes conscious of an olfactory percept only when the representation is part of a wider network involving other systems (Cooney \& Gazzaniga 2003), such as motor (Mainland \& Sobel 2006) or semantic-linguistic (Herz 2003) systems. In line with this view, sensory research outside of olfaction has found evidence that beta may be involved in sensory gating (Hong et al. 2008) or in large-scale coupling for sensori-motor integration (Siegel et al. 2012).

Importantly, unlike gamma oscillations, oscillations in the beta range require participation of (at least) the piriform cortex (Neville \& Haberly 2003). (If the lateral olfactory tract is disrupted, gamma oscillations in the bulb persist; Gray \& Skinner 1988.) The higher the task demand (e.g., fine discrimination vs. simple discrimination), the higher the gamma amplitude will be in early perceptual processing (Beshel et al. 2007; Stopfer et al. 1997). Accordingly, disturbing gamma oscillations in invertebrates impairs the discrimination of similar odors (a high task demand) but does not impair the discrimination of dissimilar odors (a low task demand; Stopfer et al. 1997).

Appreciation of the long-studied oscillatory properties of the olfactory system corroborates what has been observed in other sensory modalities (cf. Fries 2005; Sauseng \& Klimesch 2008; Siegel et al. 2012; Singer 2011): (a) the synchronizations of high frequencies (e.g., gamma) in local (e.g., olfactory bulb) afferent processing (Bruns \& Eckhorn 2004; Kay \& Beshel 2010; von Stein \& Sarnthein 2000), especially when the process is challenging (e.g., fine discrimination vs. simple discrimination; Kay \& Beshel 2010); and (b) the synchronization at a somewhat slower frequency range (e.g., beta or theta) for integration within a larger-scale cognitive network (Kay et al. 2009; Key \& Beshel 2010). (For a review of the neural correlates of olfactory consciousness, see Merrick et al. [2014].)

The foregoing reveals some conceptual progress regarding the neural correlates of consciousness. As noted by Merker, it is clear that isolating the neural correlates of olfactory consciousness will require further investigation. (It should be reiterated that, for good reasons, some have claimed that cortical electroencephalography does not reflect conscious processing; Merker 2013b; 2013c.) Critical for the study of the neural correlates of consciousness, and for the cortical-subcortical controversy, is Merker's insight about the necessary role of the dorsal pulvinar in olfactory consciousness.

As is evident in the commentaries, we believe that PFT will spur the field to think about the problem of how consciousness arises from nervous function in more theoretically driven and evolutionary-based ways.

\section{R9. Conclusion}

In phylogeny, there has been a trend toward the increased compartmentalization of function (Allman 2000). Having 
different brain circuits devoted to different kinds of tasks introduces the struggle of parts problem (Mayr 2001), which occurs when the introduction of new structures such as organs involves competitive interactions with extant ones. This problem may have increased the pressure for "many-to-one" solutions, including the conscious field. From our EASE perspective, although such a solution could conceivably occur without something like consciousness, such a possibility was not selected in evolutionary history, in which problems are sometimes solved by counterintuitive and suboptimal strategies (Dawkins 1982; Gould 1977; Mayr 2001; Roe \& Simpson 1958). (We should reiterate that intuitions regarding how sensations should be mapped onto responses are actually computationally impossible; see Tsotsos [1995; 2011].)

The commentaries give us confidence that PFT, though based in part on established ideas from diverse fields of study, is a novel synthesis that advances understanding of the role of conscious states in nervous function. PFT attempts to redefine the nature of consciousness. One can propose that, if the heart can be conceptualized as a pump and the kidney as a filter, then consciousness could be conceptualized as a frame composed of tokens (e.g., the color blue, a smell, or pain) that are in a common format, a format that can be sampled only by the action systems of the skeletomotor output system. As an interface of sorts for the action system, the conscious field permits for the response to a given content to be framed by the other contents composing the field. The physical basis of the frame associated with consciousness is most likely unlike anything we currently understand.

\section{ACKNOWLEDGMENTS}

Ezequiel Morsella dedicates this Response article to Robert M. Krauss, his doctoral advisor, who taught him all of the important lessons. This research was supported by David Matsumoto and the Center for Human Culture and Behavior at San Francisco State University. All authors are grateful for the assistance of Jed Katzel, T. Andrew Poehlman, Lawrence Williams, Pat Miller, Ryan Howell, Lucia Jacobs, Allison Allen, Sabrina Bhangal, Hyein Cho, Donish Cushing, Wei Dou, Reza Ghafur, Jessica McMillin, Pooya Razavi, Zaviera Reyes, and Anthony Velasquez.

\section{NOTES}

1. One might argue that smooth muscle actions (e.g., the pupillary reflex) are not veritable forms of action and hence should not be contrasted with voluntary actions, which, to the conscious actor, feel like "real actions." However, it is important to appreciate that, to an intelligent nonhuman observer (e.g., an imaginary, extraterrestrial ethologist), events such as the pupillary reflex would be worthy of being "coded" and jotted down as actions on an observation log. To an observer that is agnostic regarding our internal states, the pupillary reflex would appear as actionlike as a wink, blink, or the movements of a finger (Skinner 1953).

2. See neural evidence for this effect in Nath and Beauchamp (2012).

3. Conscious conflicts are often between a high-level system and a low-level system, but they may also be between (a) two low-level systems, as when one is thirsty and must drink painfully cold ice water (Morsella 2005), or (b) two high-level systems, as in the incongruent condition of the Stroop task.

4. In PFT, conscious contents can be construed as action options, or, more precisely, as constraining dimensions (Morsella \& Bargh 2010b), because that which is conscious reduces the space of possible skeletomotor action selection. Unlike with the traditional (circular) definition of conscious representation (which is defined only in terms of being conscious), here the representations are defined by more than their being conscious: They are also defined by their ability to constrain action selection in the skeletomotor output system. These conscious constraining dimensions are not involved in intersensory conflicts, intrasensory conflicts, or the conflicts involving non-skeletal muscle effectors (Morsella et al. 2009a). Akin to a single steering wheel that is controlled by multiple agentic systems, the skeletomotor output system suffers from a particular kind of multi-determined guidance. Just as simple motor acts suffer from the "degrees of freedom" problem, because there are countless ways to instantiate a motor act such as grasping a handle (Rosenbaum 2002), so does action selection, for there are many action options. For action-goal selection, the challenge is met not by unconscious motor algorithms (as in the case of motor programming; Rosenbaum 2002), but by the involvement of the conscious field. In line with this view, Goodale and Milner (2004) conclude that "the primary role of [conscious] perceptual representations is not in the execution of actions, but rather in helping the person or animal arrive at a decision to act in a particular way" (p. 48).

5. Similarly, at the level of operant behavior, skeletomotor considerations are unaffected by, say, incentive states. For example, the actions of navigating through a maze or drawing a candy cane would be carried out in roughly the same manner regardless of the nature of the reward contingencies (Skinner 1953).

6. Supporting the integration consensus, findings in the field of anesthesiology suggest that anesthetic agents work on consciousness in part by halting the integration of information (Alkire et al. 2008; Lee et al. 2009; Mashour 2004; see related evidence in Boveroux et al. 2010; Långsjö et al. 2012; Lewis et al. 2012; Schroter et al. 2012; Schrouff et al. 2011). Regarding thalamic accounts of consciousness, some anesthetics can cause a reduction in thalamic blood flow and metabolism during the loss of consciousness, whereas other kinds of anesthetics result in increases in thalamic metabolism (e.g., ketamine) or decreases in metabolism while the subject remains conscious (e.g., during sevoflurane sedation; cf. Alkire et al. 2008). Additionally, studies using electroencephalography have shown that as soon as a subject loses consciousness, there is a marked change in cortical electroencephalography, while the thalamic electroencephalography remains relatively the same for some minutes afterwards. According to Alkire et al. (2008), this suggests that the thalamus may not be the sole location of consciousness. Investigations into feedforward and feed-backward connectivity while under anesthesia suggest that conscious states are associated with fronto-parietal networks (Lee et al. 2009). (See further discussion on anesthesia and consciousness in Poehlman et al. [2012].)

\section{References}

[The letters " $a$ " and " $r$ " before author's initials stand for target article and response references, respectively]

Ach, N. (1905/1951) Determining tendencies: Awareness. In: Organization and pathology of thought, ed. D. Rapaport, pp. 15-38. Columbia University Press (Original work published in 1905). [arEM]

Adolph, K. E., Cole, W. G., Komati, M., Garciaguirre, J. S., Badaly, D., Lingeman, J M., Chan, G. L. Y. \& Sotsky, R. B. (2012) How do you learn to walk? Thousands of steps and dozens of falls per day. Psychological Science 23:1387-94. [HD]

Adolph, K. E. \& Robinson, S. R. (2015) Motor development. In: Handbook of child psychology and developmental science, vol. 2, 7th edition, ed. L. Liben \& U. Muller, pp. 114-57. Wiley. [HD]

Adrian, E. D. (1942) Olfactory reactions in the brain of the hedgehog. The Journal of Physiology 100(4):459-73. [rEM]

Adrian, E. D. (1950a) Sensory discrimination: With some recent evidence from the olfactory organ. British Medical Bulletin 6(4):330-32. [rEM]

Adrian, E. D. (1950b) The electrical activity of the mammalian olfactory bulb. Electroencephalography and Clinical Neurophysiology 2(1):377-88. [rEM]

Aggleton, J. P. \& Brown, M. W. (2006) Interleaving brain systems for episodic and recognition memory. Trends in Cognitive Sciences 10(10):455-63. [MGa] 
References/Morsella et al.: Homing in on consciousness in the nervous system

Albrecht, J., Demmel, M., Schöpf, V., Kleemann, A. M., Kopietz, R., May, J., Schreder, T., Zernecke, R., Brückmann, H. \& Wiesmann, M. (2011) Smelling chemosensory signals of males in anxious versus nonanxious condition increases state anxiety of female subjects. Chemical Senses 36:19-27. doi:10.1093/ chemse/bjq087. [BM]

Aleman, B. \& Merker, B. (2014) Consciousness without cortex: A hydranencephaly family survey. Acta Paediatrica 103(10):1057-65. [aEM]

Alkire, M., Hudetz, A. \& Tononi, G. (2008) Consciousness and anesthesia. Science 322(5903):876-80. [arEM]

Allen, A. K., Wilkins, K., Gazzaley, A. \& Morsella, E. (2013) Conscious thoughts from reflex-like processes: A new experimental paradigm for consciousness research. Consciousness and Cognition 22:1318-31. [arEM]

Allman, J. M. (2000) Evolving brains. Scientific American Library. [arEM]

Allport, D. A. (1989) Visual attention. In: Foundations of cognitive science, vol. 2, ed. M. I. Posner, pp. 631-82. MIT Press. [rEM]

Anderson, A. K. \& Phelps, E. A. (2002) Is the human amygdala critical for the subjective experience of emotion? Evidence of intact dispositional affect in patients with amygdala lesions. Journal of Cognitive Neuroscience 14:709-20. [aEM]

Anderson, J. (1983) The architecture of cognition. Harvard University Press. [rEM]

Andreou, E. (2000) Bully/victim problems and their association with psychological constructs in 8- to 12-vear-old Greek schoolchildren. Aggressive Behavior 26:49-56. [FP]

Ansorge, U., Neumann, O., Becker, S., Kalberer, H. \& Cruse, H. (2007) Sensorimotor supremacy: Investigating conscious and unconscious vision by masked priming. Advances in Cognitive Psychology 3:257-74. [aEM]

Arkin, R. C. (1998) Behavior-based robotics. MIT Press. [aEM]

Aru, J. \& Bachmann, T. (2009) Occipital EEG correlates of conscious awareness when subjective target shine-through and effective visual masking are compared: Bifocal early increase in gamma power and speed-up of P1. Brain Research 1271:60-73. [arEM]

Aru, J., Bachmann, T., Singer, W. \& Melloni, L. (2012) Distilling the neural correlates of consciousness. Neuroscience and Biobehavioral Reviews 36:737-46. [aEM]

Atzil, S., Hendler, T. \& Feldman, R. (2011) Specifying the neurobiological basis of human attachment: Brain, hormones, and behavior in synchronous and intrusive mothers. Neuropsychopharmacology 36(13):2603-15. doi: 10.1038/ npp.2011.172. [JES]

Augustinova, M. \& Ferrand, L. (2014) Automaticity of word reading: Evidence from the semantic Stroop paradigm. Current Directions in Psychological Science 23:343-48. [rEM]

Baars, B. (1988) A cognitive theory of consciousness. Cambridge University Press. [arEM, SB, BH]

Baars, B. J. (1997a) Contrastive phenomenology: A thoroughly empirical approach to consciousness. In: The nature of consciousness: Phiolosophical controversies, ed. O. J. Flanagan, N. Block \& G. Guzeldere, pp. 187-202. MIT Press. [BM]

Baars, B. J. (1997b) Some essential differences between consciousness and attention, perception, and working memory. Consciousness and Cognition 6:363-71. $[\mathrm{rEM}]$

Baars, B. J. (1998) The function of consciousness: Reply. Trends in Neurosciences 21 (5):201. [aEM]

Baars, B. J. (2002) The conscious access hypothesis: Origins and recent evidence. Trends in Cognitive Sciences 6(1):47-52. [arEM]

Baars, B. J. (2005) Global workspace theory of consciousness: Toward a cognitive neuroscience of human experience. Progress in Brain Research 150:45-53. [aEM, LM]

Baddeley, A. D. (2007) Working memory, thought and action. Oxford University Press. [aEM]

Bak, P. (1996) How nature works: The science of self-organized criticality. Copernicus. $[\mathrm{CH}]$

Baker, T. B., Piper, M. E., McCarthy, D. E., Majeskie, M. R. \& Fiore, M. C. (2004) Addiction motivation reformulated: An affective processing model of negative reinforcement. Psychological Review 111:33-51. [rEM]

Balcetis, E. \& Dunning, D. (2010) Wishful seeing: More desired objects are seen as closer. Psychological Science 21(1):147-52. doi: 10.1177/0956797609356283. [AKS]

Banerjee, P., Chatterjee, P. \& Sinha, J. (2012) Is it light or dark? Recalling moral behavior changes perception of brightness. Psychological Science 23:407409. [aEM]

Banks, W. P. (1995) Evidence for consciousness. Consciousness and Cognition 4:270-72. [arEM]

Bar, M., Kassam, K. S., Ghuman, A. S., Boshyan, J., Schmid, A. M., Dale, A. M., Hämäläinen, M. S., Marinkovic, K., Schacter, D. L., Rosen, B. R. \& Halgren, E. (2006) Top-down facilitation of visual recognition. Proceedings of the National Academy of Sciences USA 103:449-54. [LP, rEM]

Bargh, J. A. (2014) Our unconscious mind. Scientific American 30:30-37. doi:10.1038/scientificamerican0114-30. [FP]
Bargh, J. A. \& Chartrand, T. L. (1999) The unbearable automaticity of being. American Psychologist 54(7):462-79. [FP]

Bargh, J. A. \& Morsella, E. (2008) The unconscious mind. Perspectives on Psychological Science 3:73-79. [arEM]

Bargh, J. A., Chen, M. \& Burrows, L. (1996) Automaticity of social behavior: Direct effects of trait construct and stereotype activation on action. Journal of Personality and Social Psychology 71:230-44. [FP]

Bargh, J. A. \& Pietromonaco, P. (1982) Automatic information processing and social perception: The influence of trait information presented outside of conscious awareness on impression formation. Journal of Personality and Social Psychology 43:437-49. [FP]

Baron, R. S., Inman, M. L., Kao, C. F. \& Logan, H. (1992) Negative emotion and superficial social processing. Motivation and Emotion 16:323-46. [FP]

Barr, M. L. \& Kiernan, J. A. (1993) The human nervous system: An anatomical viewpoint, 6th edition. Lippincott. [aEM]

Barrett, H. C. (2005) Enzymatic computation and cognitive modularity. Mind and Language 20:259-87. [aEM]

Barrett, L. F. (2006) Are emotions natural kinds? Perspectives on Psychological Science 1(1):28-58. [HLM]

Barsalou, L. W. (1999) Perceptual symbol systems. Behavioral and Brain Sciences 22:577-609. [aEM]

Basso, D., Bisiacchi, P. S., Cotelli, M. \& Farinello, C. (2001) Planning times during Travelling Salesman's problem: Differences between closed head injury and normal subjects. Brain and Cognition 46(1-2):38-42. [DB]

Basso, D., Lotze, M., Vitale, L., Ferreri, F., Bisiacchi, P. S., Olivetti Belardinelli, M., Rossini, P. M. \& Birbaumer, N. (2006) The role of prefrontal cortex in visuospatial planning: A repetitive-TMS study. Experimental Brain Research 171 (3):411-15. [DB]

Baumeister, R. F. \& Bargh, J. A. (2014) Conscious and unconscious: Toward an integrative understanding of human mental life and action. In: Dual process theories of the social mind, ed. J. W. Sherman, B. Gawronski, \& Y. Trope, Ch. 3 , pp. 35-39. Guilford Press. [BH]

Baumeister, R. F. \& Masicampo, E. J. (2010) Conscious thought is for facilitating social and cultural interactions: How mental simulations serve the animalculture interface. Psychological Review 117(3):945-71. [BH, AV]

Baumeister, R. F. \& Vohs, K. D. (2004) Handbook of self-regulation: Research, theory, and applications. Guilford Press. [rEM]

Baumeister, R. F., Vohs, K. D., DeWall, N. \& Zhang, L. (2007) How emotion shapes behavior: Feedback, anticipation, and reflection, rather than direct causation. Personality and Social Psychology Review 11:167-203. [aEM]

Bayne, T. (2013) Agency as a marker of consciousness. In: Decomposing the will, ed. A Clark, T. Vierkant \& J. Kiverstein, pp. 160-81. Oxford University Press. [aEM]

Bednark, J. G. \& Franz, E. A. (2014) Agency attribution: Event-related potentials and outcome monitoring. Experimental Brain Research 232:1117-26. [EAF]

Bednark, J. G., Reynolds, J. N. J, Stafford, T., Redgrave, P. \& Franz, E. A. (2013) Creating a movement heuristic for voluntary action: Electrophysiological correlates of movement-outcome learning. Cortex 49(3):771-80. [EAF]

Behrendt, R. P. (2013) Conscious experience and episodic memory: Hippocampus at the crossroads. Frontiers in Psychology 4, article 304. (Online journal). doi:10.3389/fpsyg.2013.00304. [RL]

Bellebaum, C., Koch, B., Schwarz, M. \& Daum, I. (2008) Focal basal ganglia lesions are associated with impairments in reward-based reversal learning. Brain 131:829-41. [aEM]

Bennett, M. R. \& Hacker, P. M. S. (2003) Philosophical foundations of neuroscience. Blackwell. [SB]

Berkowitz, L. (1993) Toward a general theory of anger and emotional aggression: Implications of the cognitive-neoassociationistic perspective for the analysis of anger and other emotions. In: Advances in social cognition, vol. 6, ed. R. S. Wyer \& T. K. Srull, pp. 1-46. Erlbaum. [FP]

Berti, A. \& Pia, L. (2006) Understanding motor awareness through normal and pathological behavior. Current Directions in Psychological Science 15:24550. [aEM]

Beshel, J., Kopell, N. \& Kay, L. M. (2007) Olfactory bulb gamma oscillations are enhanced with task demands. Journal of Neuroscience 27:8358-65. [rEM]

Betts, G. H. (1909/1972) The distribution and functions of mental imagery. AMS Press. (Original work published in 1909 as author's thesis for Columbia University). 1972 reprint edition issued in series: Teachers College, Columbia University. Contributions to Education, No. 26. [aEM]

Bhalla, M. \& Proffitt, D. R. (1999) Visual-motor recalibration in geographical slant perception. Journal of Experimental Psychology: Human Perception and Performance 25:1076-96. [aEM]

Bhangal, S., Cho, H., Geisler, M. W. \& Morsella, E. (2016) The prospective nature of voluntary action: Insights from the reflexive imagery task. Review of General Psychology 20:101-17. [rEM]

Bhat, A. N. \& Galloway, J. C. (2006) Toy-oriented changes during early arm movements: Hand kinematics. Infant Behavior and Development 29:358-72. [HD] 
References/Morsella et al.: Homing in on consciousness in the nervous system

Bindra, D. (1974) A motivational view of learning, performance, and behavior modification. Psychological Review 81:199-213. [arEM]

Bindra, D. (1978) How adaptive behavior is produced: A perceptual-motivational alternative to response-reinforcement. Behavioral and Brain Sciences 1:4191. [arEM]

Bizzi, E. \& Mussa-Ivaldi, F. A. (2004) Toward a neurobiology of coordinate transformations. In: The cognitive neurosciences III, ed. M. S. Gazzaniga, pp. 41325. MIT Press. [aEM]

Bjorklund, D. F. \& Pellegrini, A. D. (2002) Child development and evolutionary psychology. Child Development 71(6):1687-708. doi:10.1111/1467-8624.00258. PMID 11194266. [FP]

Bjorkman, M., Juslin, P. \& Winman, A. (1993) Realism of confidence in sensory discrimination: The underconfidence phenomenon. Perception and Psychophysics 54(1):75-81. [ZL]

Blackmore, S. (2011) Consciousness: An introduction. Oxford University Press. [SB]

Blake, R. \& Logothetis, N. K. (2002) Visual competition. Nature Reviews Neuroscience $3: 13-21$. [aEM]

Blakemore, S. J. \& Choudhury, S. (2006) Development of the adolescent brain: Implications for executive function and social cognition. Journal of Child Psychology and Psychiatry 47:296-312. [HD]

Blanken, G., Wallesch, C.-W. \& Papagno, C. (1990) Dissociations of language functions in aphasics with speech automatisms (recurring utterances). Cortex 26:41-63. [aEM]

Bleuler, E. (1924) Textbook of psychiatry, trans. A. A. Brill. Macmillan. [aEM, SB]

Block, N. (1995a) How many concepts of consciousness? (Response article) Behavioral and Brain Sciences 18(2):272-87. [AV]

Block, N. (1995b) On a confusion about a function of consciousness. (Target article) Behavioral and Brain Sciences 18(2):227-47; discussion 247-87. [arEM]

Block, N. (2007) Consciousness, accessibility, and the mesh between psychology and neuroscience. Behavioral and Brain Sciences 30:481-548. [rEM]

Block, N. (2009) Comparing the major theories of consciousness. In: The cognitive neurosciences $I V$, ed. M. Gazzaniga, pp. 1111-22. MIT Press. [BLS]

Boal, J. G., Dunham, A. W., Williams, K. T. \& Hanlon, R. T. (2000) Experimental evidence for spatial learning in octopuses (Octopus bimaculoides). Journal of Comparative Psychology 114:246-52. [DAR]

Bodenhausen, G. V., Kramer, G. P. \& Süsser, K. (1994) Happiness and stereotypic thinking in social judgment. Journal of Personality and Social Psychology 66:621-32. [FP]

Boireau, N., Pinault, G., Kirsche, L. \& MacLeod, P. (2000) Lateralization of vanillin odor perception by birhinal stimulation: Differential olfactometer method. In: Abstracts, ISOT/ECRO 2000 Congress: Proceedings of the 14th International Symposium on Olfaction and Taste (ISOT), July 20-24, 2000, Brighton, U.K. European Chemoreception Research Organisation (ECRO). [BM]

Boly, M., Garrido, M. I., Gosseries, O., Bruno, M. A., Boveroux, P., Schnakers, C., Massimini, M., Litvak, V., Laureys, S. \& Friston, K. (2011) Preserved feedforward but impaired top-down processes in the vegetative state. Science 332:85862. $[\mathrm{aEM}]$

Bornstein, M. H., Tamis-LeMonda, C. S., Tal, J., Ludemann, P., Toda, S., Rahn, C. W., Pêcheux, M. G., Azuma, H. \& Vardi, D. (1992) Maternal responsiveness to infants in 3 societies - the United States, France, and Japan. Child Development 63(4):808-21. [JES]

Boulton, M. J. \& Smith, P. K. (1994) Bully/victim problems in middle-school children: Stability, self-perceived competence, peer perceptions and peer acceptance. British Journal of Development Psychology 12:315-29. [FP]

Boveroux, P., Vanhaudenhuyse, A., Bruno, M. A., Noirhomme, Q., Lauwick, S., Luxen, A., Degueldre, C., Plenevaux, A., Schnakers, C., Phillips, C., Brichant, J F., Bonhomme. V., Maquet, P., Greicius, M. D., Laureys, S. \& Boly, M. (2010) Breakdown of within- and between-network resting state functional magnetic resonance imaging connectivity during propofol-induced loss of consciousness. Anesthesiology 113:1038-53. [arEM]

Bowen, S. \& Marlatt, A. (2009) Surfing the urge: Brief mindfulness-based intervention for college student smokers. Psychology of Addictive Behaviors 23 (4):666-71. [BH]

Bowlby, J. (1969) Attachment and loss, vol. 1. Attachment. Hogarth Press. [JES]

Bowlby, J. (1973) Attachment and loss, vol. 2. Separation: Anxiety and anger. Basic Books. [JES]

Brendel, B., Hertrich, I., Erb, M., Lindner, A., Riecker, A., Grodd, W. \& Ackermann, H. (2010) The contribution of mesiofrontal cortex to the preparation and execution of repetitive syllable productions: An fMRI study. Neuroimage 50 (3):1219-30. doi: 10.1016/j.neuroimage.2010.01.039. [JES]

Brentano, F. (1874) Psychology from an empirical standpoint. Oxford University Press. [aEM]

Bridgeman, B. (1973) Receptive fields in single cells of monkey visual cortex during visual tracking. International Journal of Neurosciences 6:141-52. [BB]

Brower, D. (1947) The experimental study of imagery: II. The relative predominance of various imagery modalities. Journal of General Psychology 37:199200. [aEM]
Brown, S. L. \& Brown, R. M. (2015) Connecting prosocial behavior to improved physical health: Contributions from the neurobiology of parenting. Neuroscience and Biobehavioral Reviews 55:1-17. doi: 10.1016/j.neubiorev.2015.04.004. [JES]

Bruner, J. S. (1973) Going beyond the information given: Studies in the psychology of knowing. Norton. [aEM]

Bruner, J. S. \& Postman, L. (1949) On the perception of incongruity: A paradigm. Journal of Personality 18:206-23. [aEM]

Bruns, A. \& Eckhorn, R. (2004) Task-related coupling from high-to low-frequency signals among visual cortical areas in human subdural recordings. International Journal of Psychophysiology 51(2):97-116. [rEM]

Bryon, S. \& Jedynak, C. P. (1972) Troubles du transfert interhemispherique: A propos de trois observations de tumeurs du corps calleux. Le signe de la main etrangère. Revue Neurologique 126:257-66. [aEM]

Buchsbaum, B. R. (2013) The role of consciousness in the phonological loop: Hidden in plain sight. Frontiers in Psychology 4, article 496. (Online publication, Open Access e- journal). doi:10.3389/fpsyg.2013.00496. [aEM]

Buchsbaum, B. R. \& D'Esposito, M. (2008) The search for the phonological store: From loop to convolution. Journal of Cognitive Neuroscience 20:762-78. [aEM]

Buck, L. B. (2000) Smell and taste: The chemical senses. In: Principles of neural science, 4th edition, ed. E. R. Kandel, J. H. Schwartz \& T. M. Jessell, pp. 62547. McGraw-Hill. [aEM]

Bullock, D. \& Grossberg, S. (1988) Neural dynamics of planned arm movements: Emergent invariants and speed-accuracy properties during trajectory formation. Psychological Review 95:49-90. [aEM]

Butler, A. B. (2008) Evolution of the thalamus: A morphological and functional review. Thalamus and Related Systems 4:35-58. [JdV]

Buzsáki, G. (2006) Rhythms of the brain. Oxford University Press. [arEM]

Calvo-Merino, B., Glaser, D. E., Grèzes, J., Passingham, R. E. \& Haggard, P. (2005) Action observation and acquired motor skills: An FMRI study with expert dancers. Cerebral Cortex15(8):1243-49. [JSJ]

Campbell, B. A. \& Misanin, J. R. (1969) Basic drives. Annual Review of Psychology 20:57-84. [aEM]

Carlesimo, G. A., Lombardi, M. G., Caltagirone, C. \& Barban, F. (2015) Recollection and familiarity in the human thalamus. Neuroscience and Biobehavioral Reviews 54:18-28. [MGa]

Carlson, N. R. (1994) Physiology of behavior. Allyn and Bacon. [arEM]

Carmichael, S. T., Clugnet, M.-C. \& Price, J. L. (1994) Central olfactory connections in the macaque monkey. Journal of Comparative Neurology 346:403-34. $[\mathrm{BM}]$

Carver, C. S. \& Scheier, M. F. (1999) Themes and issues in the self-regulation of behavior. In: Advances in social cognition, vol. 12, ed. R. S. Wyer \& T. K. Srull, pp. 1-105. Erlbaum. [FP]

Cazzato, V., Basso, D., Cutini, S. \& Bisiacchi, P. S. (2010) Gender differences in visuospatial planning: An eye movements study. Behavioural Brain Research 206(2):177-83. [DB]

Chalmers, D. (1995) Facing up to the problem of consciousness. Journal of Consciousness Studies 2(3):200-19. [SB]

Chambon, V., Wenke, D., Fleming, S. M., Prinz, W. \& Haggard, P. (2013) An online neural substrate for sense of agency. Cerebral Cortex 23:1031-37. [aEM]

Chan, J.-L. \& Ross, E. D. (1997) Alien hand syndrome: Influence of neglect on the clinical presentation of frontal and callosal variants. Cortex 33:287-99. [aEM]

Chen, M. \& Bargh, J. A. (1997) On the automaticity of self-fulfilling prophecies: The nonconscious effects of stereotype activation on social interaction. Journal of Experimental Social Psychology 33:541-60. [FP]

Cho, H., Zarolia, P., Gazzaley, A. \& Morsella, E. (2016) Involuntary symbol manipulation (Pig Latin) from external control: Implications for thought suppression. Acta Psychologica 166:37-41. [rEM]

Chomsky, N. (1988) Language and problems of knowledge: The Managua lectures. MIT Press. [aEM]

Christensen, M. S., Lundbye-Jensen, J., Geertsen, S. S., Petersen, T. H., Paulson, O. B. \& Nielsen, J. B. (2007) Premotor cortex modulates somatosensory cortex during voluntary movements without proprioceptive feedback. Nature Neuroscience 10:417-19. [aEM]

Cicerone, K. D. \& Tanenbaum, L. N. (1997) Disturbance of social cognition after traumatic orbitofrontal brain injury. Archives of Clinical Neuropsychology 12:173-88. [aEM]

Cisek, P. (2007) Cortical mechanisms of action selection: The affordance competition hypothesis. Philosophical Transactions of the Royal Society B 362:158599. [rEM]

Cisek, P. \& Kalaska, J. F. (2010) Neural mechanisms for interacting with a world full of action choices. Annual Review of Neuroscience 33:269-98. [rEM]

Clark, A. (2002) Is seeing all it seems? Action, reason and the grand illusion. Journal of Consciousness Studies 9:181-202. [arEM]

Clark, A. (2013) Whatever next? Predictive brains, situated agents, and the future of cognitive science. Behavioral and Brain Sciences 36(3):181-204. Available at: http://doi.org/10.1017/S0140525X12000477 [HLM, AKS] 
References/Morsella et al.: Homing in on consciousness in the nervous system

Cleeremans, A. E. (2003) The unity of consciousness: Binding, integration, and dissociation. Oxford University Press. [SB]

Coenen, A. M. L. (1998) Neuronal phenomena associated with vigilance and consciousness: From cellular mechanisms to electroencepalographic patterns. Consciousness and Cognition 7:42-53. [aEM]

Cohen, J. D., Dunbar, K. \& McClelland, J. L. (1990) On the control of automatic processes: A parallel distributed processing account of the Stroop effect. Psychological Review 97:332-61. [aEM]

Cohen, M. A., Alvarez, G. A. \& Nakayama, K. (2011) Natural-scene perception requires attention. Psychological Science 22:1165-72. [PED]

Cohen, M. A., Cavanagh, P., Chun, M. M. \& Nakayama, K. (2012) The attentional requirements of consciousness. Trends in Cognitive Sciences 16:411-17. [PED, rEM]

Cohen, M. A. \& Dennett, D. C. (2011) Consciousness cannot be separated from function. Trends in Cognitive Sciences 15:358-64. [aEM]

Cohen-Kdoshay, O. \& Meiran, N. (2009) The representation of instructions operates like a prepared reflex: Flanker compatibility effects found in the first trial following S-R instructions. Experimental Psychology 56:128-33. [aEM]

Coles, M. G. H., Gratton, G., Bashore, T. R., Eriksen, C. W. \& Donchin, E. (1985) A psychophysiological investigation of the continuous flow model of human information processing. Journal of Experimental Psychology: Human Perception and Performance 11:529-53. [aEM]

Conant, R. \& Ashby, W. R. (1970) Every good regulator of a system must be a model of that system. International Journal of Systems Science 1(2):89-97. [AKS]

Cooney, J. W. \& Gazzaniga, M. S. (2003) Neurological disorders and the structure of human consciousness. Trends in Cognitive Sciences 7:161-66. [arEM]

Cooper, A. D., Sterling, C. P., Bacon, M. P. \& Bridgeman, B. (2012) Does action affect perception or memory? Vision Research 62:235-40. [arEM]

Corbetta, M., Patel, G. H. \& Shulman, G. L. (2008) The reorienting system of the human brain: From environment to theory of mind. Neuron 58:306-24. [PED]

Corr, P. J. \& Morsella, E. (2015) The conscious control of behavior: Revisiting Gray's comparator model. In: Personality and control, vol. 4, ed. P. J. Corr, M. Fajkowska, M. W. Eysenck \& A. Wytykowska, pp. 15-42. Eliot Werner. [rEM]

Crick, F. (1995) The astonishing hypothesis: The scientific search for the soul. Touchstone. [aEM]

Crick, F. \& Koch, C. (1990) Toward a neurobiological theory of consciousness. Seminars in the Neurosciences 2:263-75. [arEM]

Crick, F. \& Koch, C. (1995) Are we aware of neural activity in primary visual cortex? Nature 375:121-23. [aEM]

Crick, F. \& Koch, C. (1998) Consciousness and neuroscience. Cerebral Cortex 8:97107. [aEM]

Crick, F. \& Koch, C. (2000) The unconscious homunculus. In: Neural correlates of consciousness, ed. T. Metzinger, pp. 103-10. MIT Press. [rEM]

Crick, F. \& Koch, C. (2003) A framework for consciousness. Nature Neuroscience 6:1-8. [aEM, PED]

Crook, C. K. (1978) Taste perception in the newborn infant. Infant Behavior and Development 1:52-69. [HD]

Cross, S. E. \& Markus, H. R. (1990) The willful self. Personality and Social Psychology Bulletin 16:726-42. [FP]

Cunningham, W. A. \& Kirkland, T. (2012) Emotion, cognition, and the classical elements of mind. Emotion Review 4(4):369-70. Available at: http://doi.org/ 10.1177/1754073912445820 [HLM]

Cunningham, W. A., Dunfield, K. A. \& Stillman, P. E. (2013) Emotional states from affective dynamics. Emotion Review 5(4):344-55. Available at: http://doi.org/ $10.1177 / 1754073913489749$ [HLM]

Curtis, C. E. \& D'Esposito, M. (2009) The inhibition of unwanted actions. In: Oxford handbook of human action, ed. E. Morsella, J. A. Bargh \& P. M. Gollwitzer, pp. 72-97. Oxford University Press. [aEM]

Damasio, A. R. (1989) Time-locked multiregional retroactivation: A systems-level proposal for the neural substrates of recall and recognition. Cognition 33:2562. $[\mathrm{aEM}]$

Damasio, A. R. (1994) Descartes' error. Macmillan. [AKS]

Damasio, A. R. (1999) The feeling of what happens: Body and emotion in the making of consciousness. Harvest Books. [aEM, AV]

Damasio, A. R. (2010) Self comes to mind: Constructing the conscious brain. Pantheon. [aEM]

Damasio, A. R., Damasio, H. \& Tranel, D. (2012) Persistence of feelings and sentience after bilateral damage of the insula. Cerebral Cortex 23:833-46. [aEM]

Damasio, A. R., Tranel, D. \& Damasio, H. C. (1991) Somatic markers and the guidance of behavior: Theory and preliminary testing. In: Frontal lobe function and dysfunction, ed. H. S. Levin, H. M. Eisenberg \& A. L. Benton, pp. 217-29. Oxford University Press. [rEM]

David, N., Newen, A. \& Vogeley, K. (2008) The "sense of agency" and its underlying cognitive and neural mechanisms. Consciousness and Cognition 17:523-34. $[\mathrm{aEM}]$
Dawkins, R. (1982) The extended phenotype: The long reach of the gene. Oxford University Press. [rEM]

De la Cruz, V., Rodriguez-Ortiz, C. J., Balderas, I. \& Bermudez-Rattoni, F. (2008) Medial temporal lobe structures participate differentially in consolidation of safe and aversive taste memories. European Journal of Neuroscience 28:137781. $[\mathrm{MGa}]$

Dehaene, S. (2009) Neuronal global workspace. In: The Oxford companion to consciousness, ed. T. Bayne, A. Cleeremans \& P. Wilken, pp. 466-70. Oxford University Press. [SB]

Dehaene, S. (2014) Consciousness and the brain: Deciphering how the brain codes our thoughts. Viking. [arEM]

Dehaene, S. \& Changeux, J. P. (2011) Experimental and theoretical approaches to conscious processing. Neuron 70(2):200-27. [LM]

Dehaene, S. \& Naccache, L. (2001) Towards a cognitive neuroscience of consciousness: Basic evidence and a workspace framework. Cognition 79(1-2):137. [aEM, SB, LM]

Dehaene, S., Changeux, J.-P., Naccache, L., Sackur, J. \& Sergent, C. (2006) Conscious, preconscious, and subliminal processing: A testable taxonomy. Trends in Cognitive Sciences 10:204-11. [BH]

Del Cul, A., Baillet, S. \& Dehaene, S. (2007) Brain dynamics underlying the nonlinear threshold for access to consciousness. PLoS Biology 5:e260. [aEM]

Dennett, D. C. (1991) Consciousness explained. Little, Brown. [arEM, SB]

Dennett, D. C. (2005) Sweet dreams. MIT Press. [SB]

Denton, D. A. (1982) The hunger for salt: An anthropological, physiological and medical analysis. Springer. [RL]

Desender, K., van Opstal, F. V. \& van den Bussche, E. (2014) Feeling the conflict The crucial role of conflict experience in adaptation. Psychological Science 25:675-83. [arEM]

Desmurget, M., Reilly, K. T., Richard, N., Szathmari, A., Mottolese, C. \& Sirigu, A. (2009) Movement intention after parietal cortex stimulation in humans. Science 324(5928):811-13. [aEM]

Desmurget, M. \& Sirigu, A. (2010) A parietal-premotor network for movement intention and motor awareness. Trends in Cognitive Sciences 13:411-19. [aEM]

DeSoto, M. C., Fabiani, M., Geary, D. C. \& Gratton, G. (2001) When in doubt, do it both ways: Brain evidence of the simultaneous activation of conflicting responses in a spatial Stroop task. Journal of Cognitive Neuroscience 13:523-36. [aEM]

Deubel, H., Bridgeman, B. \& Schneider, W. X. (1998) Immediate post-saccadic information mediates space constancy. Vision Research 38:3147-59. [BB]

Devine, P. G. (1989) Stereotypes and prejudice: Their automatic and controlled components. Journal of Personality and Social Psychology 56:680-90. [FP]

de Waal, F. B. M. (2002) Evolutionary psychology: The wheat and the chaff. Current Directions in Psychological Science 11:187-91. [aEM]

Dietrich, E. \& Markman, A. B. (2003) Discrete thoughts: Why cognition must use discrete representations. Mind and Language 18:95-119. [aEM]

Di Lollo, V., Enns, J. T. \& Rensink, R. A. (2000) Competition for consciousness among visual events: The psychophysics of reentrant visual pathways. Journal of Experimental Psychology: General 129:481-507. [aEM, PED]

Doesburg, S. M., Green, J. L., McDonald, J. J. \& Ward, L. M. (2009) Rhythms of consciousness: Binocular rivalry reveals large-scale oscillatory network dynamics mediating visual perception. PLoS ONE 4:e0006142. [arEM]

Doesburg, S. M., Kitajo, K. \& Ward, L. M. (2005) Increased gamma-band synchrony precedes switching of conscious perceptual objects in binocular rivalry. $\mathrm{Neu}$ roReport 16:1139-42. [arEM]

Doherty, M. J., Wilensky, A. J., Holmes, M. D., Lewis, D. H., Rae, J. \& Cohn, G. H (2002) Singing seizures. Neurology 59:1435-38. [aEM]

Duncker, K. (1939) The influence of past experience upon perceptual properties. American Journal of Psychology 52(2):255-65. [DB]

Duprez, T. P., Serieh, B. A. \& Reftopoulos, C. (2005) Absence of memory dysfunction after bilateral mammillary body and mammillothalamic tract electrode implantation: Preliminary experience in three patients. American Journal of Neuroradiology 26:195-98. [aEM]

Edelman, G. M. (1989) The remembered present. Basic Books. [aEM]

Edelman, G. M. (2004) Wider than the sky: The phenomenal gift of consciousness. Yale University Press. [AV]

Edelman, G. M. \& Tononi, G. (2000) A universe of consciousness: How matter becomes imagination. Basic Books. [aEM]

Eeckman, F. H. \& Freeman, W. J. (1990) Correlations between unit firing and EEG in the rat olfactory system. Brain Research 528(2):238-44. [rEM]

Ehrsson, H. H. (2007) The experimental induction of out-of-body experiences. Science 317(5841):1048. [arEM]

Eichenbaum, H., Shedlack, K. J. \& Eckmann, K. W. (1980) Thalamocortical mechanisms in odor-guided behavior. Brain, Behavior and Evolution 17:25575. $[\mathrm{aEM}]$

Eimer, M. \& Schlaghecken, F. (2003) Response facilitation and inhibition in subliminal priming. Biological Psychology 64(1):7-26. [AA, rEM]

Einstein, A. \& Infeld, L. (1938/1967) The evolution of physics. Cambridge University Press/Touchstone. (Original work published in 1938). [arEM] 
References/Morsella et al.: Homing in on consciousness in the nervous system

Ekman, P. (1992) An argument for basic emotions. Cognition and Emotion 6(34):169-200. Available at: http://doi.org/10.1080/02699939208411068 [HLM]

Eliot, T. S. (1943) Four Quartets. Harcourt. [HSP]

Ellis, R. (2009) Interactions between action and visual objects. In: Oxford handbook of human action, ed. E. Morsella, J. A. Bargh \& P. M. Gollwitzer, pp. 214-24. Oxford University Press. [aEM]

Ellsworth, P. C. \& Scherer, K. R. (2003) Appraisal processes in emotion. In: Handbook of affective sciences, ed. R. J. Davidson, K. R. Scherer \& H. H. Goldsmith, pp. 572-95. Oxford University Press. [HLM]

Enatsu, R., Hantus, S., Gonzalez-Martinez, J. \& So, N. (2011) Ictal singing due to left frontal lobe epilepsy: A case report and review of the literature. Epilepsy and Behavior 22:404-406. [aEM]

Engel, A. K. \& Singer, W. (2001) Temporal binding and the neural correlates of sensory awareness. Trends in Cognitive Sciences 5:16-25. [arEM]

Engel, A. K., Fries, P., König, P., Brecht, M. \& Singer, W. (1999) Temporal binding, binocular rivalry, and consciousness. Consciousness and Cognition 8(2):12851. [LM]

Enns, J. T. \& Di Lollo, V. (1997) Object substitution: A new form of masking in unattended visual locations. Psychological Science 8:135-39. [PED]

Eriksen, B. A. \& Eriksen, C. W. (1974) Effects of noise letters upon the identification of a target letter in a nonsearch task. Perception and Psychophysics 16:14349. [aEM]

Eriksen, C. W. \& Schultz, D. W. (1979) Information processing in visual search: A continuous flow conception and experimental results. Perception and Psychophysics 25:249-63. [arEM]

Esposito, G., Nakazawa, J., Venuti, P. \& Bornstein, M. H. (2013) Componential deconstruction of infant distress vocalizations via tree-based models: A study of cry in autism spectrum disorder and typical development. Research in Developmental Disabilities 34(9):2717-24. doi: 10.1016/j.ridd.2013.05.036. [JES]

Fahrenfort, J. J., Scholte, H. S. \& Lamme, V. A. (2007) Masking disrupts reentrant processing in human visual cortex. Journal of Cognitive Neuroscience 19:148897. [aEM]

Farrer, C., Frey, S. H., Van Horn, J. D., Tunik, E., Turk, D., Inati, S. \& Grafton, S. T. (2008) The angular gyrus computes action awareness representations. Cerebral Cortex 18:254-61. [aEM]

Fecteau, J. H., Chua, R., Franks, I. \& Enns, J. T. (2001) Visual awareness and the online modification of action. Canadian Journal of Experimental Psychology 55:104-10. [aEM]

Fehrer, E. \& Biederman, I. (1962) A comparison of reaction time and verbal report in the detection of masked stimuli. Journal of Experimental Psychology 64:12630. [aEM]

Fehrer, E. \& Raab, D. (1962) Reaction time to stimuli masked by metacontrast. Journal of Experimental Psychology 63:143-47. [aEM]

Feldman, R. (2007) Parent-infant synchrony and the construction of shared timing: Physiological precursors, developmental outcomes, and risk conditions. Journal of Child Psychology and Psychiatry 48(3-4):329-54. doi: 10.1111/j.14697610.2006.01701.x. [JES]

Feyerabend, P. (1975) Against method: Outline of an anarchistic theory of knowledge. Atlantic Highlands. [DB]

Filevich, E. \& Haggard, P. (2013) Persistence of internal representations of alternative voluntary actions. Frontiers in Psychology 4, article 202. (Online journal) doi:10.3389/fpsyg.2013.00202. [rEM]

Filimon, F., Nelson, J. D., Hagler, D. J. \& Sereno, M. I. (2007) Human cortical representations for reaching: Mirror neurons for execution, observation, and imagery. Neuroimage 37(4):1315-28. doi: 10.1016/j.neuroimage.2007.06.008. [JES]

Finberg, L., Kiley, J. \& Luttrell, C. N. (1963) Mass accidental salt poisoning in infancy: A study of a hospital disaster. Journal of the American Medical Association 184:187-90. [HD]

Fiorito, G. \& Scotto, P. (1992) Observational learning in Octopus vulgaris. Science 256:545-47. [DAR]

Firestone, C. \& Scholl, B. J. (2014) "Top-down" effects where none should be found: The El Greco fallacy in perception research. Psychological Science 25:38-46. [aEM]

Fodor, J. A. (1980) The language of thought. Harvard University Press. [aEM]

Fodor, J. A. (1983) Modularity of mind: An essay on faculty psychology. MIT Press. [aEM]

Fodor, J. A. (1998) Concepts: Where cognitive science went wrong. Oxford University Press. [aEM]

Fodor, J. A. (2001) The mind doesn't work that way: The scope and limits of computational psychology. MIT Press. [aEM]

Ford, J. M., Gray, M., Faustman, W. O., Heinks, T. H. \& Mathalon, D. H. (2005 Reduced gamma-band coherence to distorted feedback during speech when what you say is not what you hear. International Journal of Psychophysiology 57:143-50. [aEM]

Fourneret, P. \& Jeannerod, M. (1998) Limited conscious monitoring of motor performance in normal subjects. Neuropsychologia 36:1133-40. [aEM]
Franz, E. A. (1997) Spatial coupling in the coordination of complex actions. Quarterly Journal of Experimental Psychology A 50A:684-704. [EAF]

Franz, E. A. (2003) Bimanual action representation: A window to human evolution. In: Taking action: Cognitive neuroscience perspectives on intentional acts, ed. S. H. Johnston-Frey, pp. 259-88. MIT Press. [EAF]

Franz, E. A. (2006) Converging evidence of the basal ganglia's role in focused action selection and inhibition of competing information. In: Recent breakthroughs in basal ganglia research, ed. E. Bezard, Ch. 18, pp. 227-39. Nova Science. [EAF]

Franz, E. A. (2010) A framework for conceptual binding of bimanual actions: Possible applications to neurology and neuro-rehabilitative therapies. Current Trends in Neurology 4:1-22. [EAF]

Franz, E. A. (2012) The allocation of attention to learning of goal-directed actions: A cognitive neuroscience framework focusing on the basal ganglia. Frontiers in Psychology 3, article 535:1-15. doi: 10.3389/fpsyg.2012.00535. [EAF]

Franz, E. A., Eliassen, J., Ivry, R. B. \& Gazzaniga, M. S. (1996) Dissociation of spatial and temporal coupling in the bimanual movements of callosotomy patients. Psychological Science 7:306-10. [EAF]

Franz, E. A. \& Gillett, G. (2011) John Hughlings Jackson’s evolutionary neurology: A unifying framework for cognitive neuroscience. Brain 134(10):3114-20. [EAF]

Franz, E. A. \& McCormick, R. (2010) Conceptual unifying constraints on anticipatory bimanual control. Experimental Brain Research 205(2):273-82. [EAF]

Franz, E. A. \& Ramachandran, V. S. (1998) Bimanual coupling in amputees with phantom limbs. Nature Neuroscience 1:443-44. doi:10.1038/2161. [EAF]

Franz, E. A., Waldie, K. \& Smith, M. (2000) The effect of callosotomy on novel versus familiar bimanual actions: A neural dissociation between controlled and automatic processes? Psychological Science 11(1):82-85. [EAF]

Franz, E. A., Zelaznik, H. N. \& McCabe, G. (1991) Spatial topological constraints in a bimanual task. Acta Psychologica 77(2):137-51. [EAF]

Franz, E. A., Zelaznik, H. N., Swinnen, S. \& Walter, C. (2001) Spatial conceptual influences on the coordination of bimanual actions: When a dual task becomes a single task. Journal of Motor Behavior 33(1):103-12. [EAF]

Frasnelli, J., Charbonneau, G., Collignon, O. \& Lepore, F. (2009) Odor localization and sniffing. Chemichal Senses 34:139-44. [BM]

Freeman, W. J. (1975) Mass action in the nervous system: Examination of the neurophysiological basis of adaptive behavior through the EEG. Academic Press. [rEM]

Freeman, W. J. (1979) Nonlinear dynamics of paleocortex manifested in the olfactory EEG. Biological Cybernetics 35(1):21-37. [rEM]

Freeman, W. J. (1987) Nonlinear neural dynamics in olfaction as a model for cognition. In: Dynamics of sensory and cognitive processing in the brain, ed. E. Basar, pp. 19-29. Springer-Verlag. [rEM]

Freeman, W. J. (1991) The physiology of perception. Scientific American 264:7885. $[\mathrm{aEM}]$

Freeman, W. J. (2004) William James on consciousness, revisited. Chaos and Complexity Letters 1:17-42. [arEM]

Freeman, W. J. (2007) Indirect biological measures of consciousness from field studies of brains as dynamical systems. Neural Networks 20:1021-31. [rEM]

Freud, S. (1915) The unconscious. In: The Standard Edition of the Complete Psychological Works of Sigmund Freud. Vol. 14: On the history of the post psychoanalytic movement, papers on metapsychology and other works, ed. J. Strachey \& A. Freud, pp. 159-204. The Hogarth Press. [FP]

Fried, I., Katz, A., McCarthy, G., Sass, K. J., Williamson, P., Spencer, S. S. \& Spencer, D. D. (1991) Functional organization of human supplementary motor cortex studied by electrical stimulation. The Journal of Neuroscience 11 (11):3656-66. [aEM, JES]

Friedman-Hill, S. R., Robertson, L. C. \& Treisman, A. (1995) Parietal contributions to visual feature binding: Evidence from a patient with bilateral lesions. Science 269:853-55. [aEM]

Friedrich, R. W. (2011) Olfactory neuroscience: Beyond the bulb. Current Biology 21:R438-40. doi: 10.1016/j.cub.2011.04.036. [BM]

Fries, P. (2005) A mechanism for cognitive dynamics: Neuronal communication through neuronal coherence. Trends in Cognitive Sciences 9:474-80. [arEM]

Frijda, N. H. (1986) The emotions. Cambridge University Press. [GG, rEM]

Frijda, N. H., Kuipers, P. \& ter Schure, E. (1989) Relations among emotion, appraisal, and emotional action readiness. Journal of Personality and Social Psychology 57(2):212-28. Available at: http://doi.org/10.1037/0022-3514.57.2.212 [HLM]

Friston, K. J. (2009) The free-energy principle: A rough guide to the brain? Trends in Cognitive Sciences 13(7): 293-301. [AKS]

Friston, K. J. (2010) The free-energy principle: A unified brain theory? Nature Reviews: Neuroscience 11(2):127-38. Available at: http://doi.org/10.1038/ $\operatorname{nrn} 2787$ [HLM]

Friston, K. J., Daunizeau, J., Kilner, J. \& Kiebel, S. J. (2010) Action and behavior: A free-energy formulation. Biological Cybernetics 102(3):227-60. doi: 10.1007/ s00422-010-0364-z. [AKS]

Frith, C. D. (2010) What is consciousness for? Pragmatics and Cognition 18(3):497551. [arEM] 
References/Morsella et al.: Homing in on consciousness in the nervous system

Fuster, J. M. (2003) Cortex and mind: Unifying cognition. Oxford University Press. [aEM]

Gainotti, G. (2012) Unconscious processing of emotions and the right hemisphere. Neuropsychologia 50:205-18. [GG]

Gallese, V., Fadiga, L., Fogassi, L. \& Rizzolatti, G. (1996) Action recognition in the premotor cortex. Brain 119(2):593-610. [DB]

Gallistel, C. R. (1993) The organization of learning. MIT Press. [RL]

Gallo, M. \& Rolls, E. (2012) Chemosensory learning and memory. Frontiers in Systems Neuroscience 6, article 73. (Online journal). doi:10.3389/ fnsys.2012.00073. [MGa]

Gibson, E. J. \& Pick, A. D. (2000) An ecological approach to perceptual learning and development. Oxford University Press. [HD]

Gibson, J. J. (1979) The ecological approach to visual perception. Houghton Mifflin. [aEM, JdV, JSJ]

Gillett, G. \& Franz, E. A. (2013) John Hughlings Jackson: Bridging theory and clinical observation. The Lancet 381(9866):528-29. [EAF]

Glenn, L. L. \& Dement, W. C. (1981) Membrane potential and input resistance of cat spinal motoneurons in wakefulness and sleep. Behavioural Brain Research 2:231-36. [rEM]

Glover, S. (2004) Separate visual representations in the planning and control of action. Behavioral and Brain Sciences 27:3-78. [JdV]

Godwin, C. A., Gazzaley, A. \& Morsella, E. (2013) Homing in on the brain mechanisms linked to consciousness: Buffer of the perception-and-action interface. In: The unity of mind, brain and world: Current perspectives on a science of consciousness, ed. A. Pereira, Jr. \& D. Lehmann, pp. 43-76. Cambridge University Press. [arEM]

Gold, J. I. \& Shadlen, M. N. (2007) The neural basis of decision making. Annual Reviews of Neuroscience 30:535-74. [aEM]

Gómez-Chacón, B., Gámiz, F. \& Gallo, M. (2012) Basolateral amygdala lesions attenuate safe taste memory-related c-fos expression in the rat perirhinal cortex. Behavioural Brain Research 230(2):418-22. [MGa]

Gómez-Chacón, B., Morillas, E. \& Gallo, M. (2015) Altered perirhinal cortex activity patterns during taste neophobia and their habituation in aged rats. Behavioural Brain Research 281:245-49. [MGa]

Goodale, M. \& Milner, D. (2004) Sight unseen: An exploration of conscious and unconscious vision. Oxford University Press. [arEM]

Goodhew, S. C., Dux, P. E., Lipp, O. V. \& Visser, T. A. W. (2012) Understanding recovery from object substitution masking. Cognition 122:405-15. [aEM]

Gottfried, J. A. (2006) Smell: Central nervous processing. In: Taste and smell: An update, ed. T. Hummel \& A. Welge-Lüssen, pp. 44-69. Karger. [aEM]

Gottfried, J. A. \& Dolan, R. J. (2003) The nose smells what the eye sees: Crossmodal visual facilitation of human olfactory perception. Neuron 39(2):375-86. [EAF]

Gottlieb, J. \& Mazzoni, P. (2004) Neuroscience: Action, illusion, and perception. Science 303:317-18. [aEM]

Gould, S. J. (1977) Ever since Darwin: Reflections in natural history. Norton. [arEM]

Grafman, J. \& Krueger, F. (2009) The prefrontal cortex stores structured event complexes that are the representational basis for cognitively derived actions. In: Oxford handbook of human action, ed. E. Morsella, J. A. Bargh \& P. M. Gollwitzer, pp. 197-213. Oxford University Press. [aEM]

Gray, C. M. \& Skinner, J. E. (1988) Centrifugal regulation of neuronal activity in the olfactory bulb of the waking rabbit as revealed by reversible cryogenic blockade. Experimental Brain Research 69:378-86. [rEM]

Gray, J. A. (1995) The contents of consciousness: A neuropsychological conjecture. Behavioral and Brain Sciences 18:659-76. [aEM]

Gray, J. A. (2004) Consciousness: Creeping up on the hard problem. Oxford University Press. [aEM]

Gray, J. R., Bargh, J. A. \& Morsella, E. (2013) Neural correlates of the essence of conscious conflict: fMRI of sustaining incompatible intentions. Experimental Brain Research 229:453-65. [aEM]

Graziano, M. S. A. (2008) The intelligent movement machine: An ethological perspective on the primate motor system. Oxford University Press. [aEM]

Graziano, M. S. A. (2010) God, soul, mind, brain: A neuroscientist's reflections on the spirit world. A LeapSci Book. [aEM]

Graziano, M. S. A. (2013) Consciousness and the social brain. Oxford University Press. [WP]

Greene, E. \& Ogden, R. T. (2012) Evaluating the contribution of recognition using the minimal transient discrete cue protocol. Behavioral Brain Functions 8:5367. $[\mathrm{MGu}]$

Greene, E. \& Visani, A. (2015) Recognition of letters displayed as briefly flashed dot patterns. Attention, Perception, and Psychophysics 77(6):1955-69. doi: 10.3758/ s13414-015-0913-6. [MGu]

Greenwald, A. G. (1970) Sensory feedback mechanisms in performance control: With special reference to the ideomotor mechanism. Psychological Review 77:73-99. [aEM]

Greenwald, A. G. (1972) On doing two things at once: Time sharing as a function of ideomotor compatibility. Journal of Experimental Psychology 94(1):52-57. $[\mathrm{EAF}]$
Greenwald, A. G. \& Banaji, M. R. (1995) Implicit social cognition: Attitudes, selfesteem, and stereotypes. Psychological Review 102(1):4-27. [FP]

Greenwald, A. G. \& Pratkanis, A. R. (1984) The self. In: Handbook of social cognition, ed. R. S. Wyer \& T. K. Srull, pp. 129-78. Erlbaum. [aEM]

Grillner, S., Wallen, P., Saitoh, K., Kozlov, A. \& Robertson, B. (2008) Neural bases of goal-directed locomotion in vertebrates. An overview. Brain Research Reviews 57:2-12. [HSP]

Grossberg, S. (1987) The adaptive brain, vol. 1. North Holland. [aEM]

Grossberg, S. (1988) Neural networks and natural intelligence. MIT Press. [LP]

Grossberg, S. (1999) The link between brain learning, attention, and consciousness Consciousness and Cognition 8(1):1-44. [arEM, EAF]

Grossberg, S. \& Levine, D. S. (1987) Neural dynamics of attentionally modulated Pavlovian conditioning: Blocking, inter-stimulus interval, and secondary reinforcement. Psychobiology 15(3):195-240. [LP]

Gur, M. (2015) Space reconstruction by primary visual cortex: A parallel non-computational mechanism of object representation. Trends in Neuroscience 38:20716. $[\mathrm{MGu}]$

Gur, M. \& Snodderly, D. M. (1997) A dissociation between brain activity and perception: Chromatically opponent cortical neurons signal chromatic flicker that is not perceived. Vision Research 37:377-82. [MGu]

Haberly, L. B. (1998) Olfactory cortex. In: The synaptic organization of the brain, 4th edition, ed. G. M. Shepherd, pp. 377-416. Oxford University Press. [aEM]

Haggard, P. (2005) Conscious intention and motor cognition. Trends in Cognitive Sciences 9:290-95. [aEM]

Haggard, P. (2008) Human volition: Towards a neuroscience of will. Nature Reviews: Neuroscience 9(12):934-46. doi: 10.1038/nrn2497. [aEM, JES]

Haggard, P., Aschersleben, G., Gehrke, J. \& Prinz, W. (2002a) Action, binding and awareness. In: Common mechanisms in perception and action: Attention and performance, vol. XIX, ed. W. Prinz \& B. Hommel, pp. 266-85. Oxford University Press. $[\mathrm{aEM}]$

Haggard, P., Clark, S. \& Kalogeras, J. (2002b) Voluntary action and conscious awareness. Nature Neuroscience 5(4):382-85. doi: 10.1038/nn827. [AKS]

Haidt, J. (2001) The emotional dog and its rational tail: A social intuitionist approach to moral judgment. Psychological Review 108:814-34. [rEM]

Hallett, M. (2007) Volitional control of movement: The physiology of free will. Clinical Neurophysiology 117:1179-92. [aEM]

Hallett, P. E. (1978) Primary and secondary saccades to goals defined by instructions. Vision Research 18:1279-96. [rEM]

Hameroff, S. (2010) The "conscious pilot"- dendritic synchrony moves through the brain to mediate consciousness. Journal of Biological Physics 36:71-93. $[\operatorname{arEM}]$

Hamker, F. H. (2003) The reentry hypothesis: Linking eye movements to visual perception. Journal of Vision 11:808-16. [aEM]

Harleß, E. (1861) Der Apparat des Willens [The apparatus of the will]. Zeitshrift für Philosophie und philosophische Kritik 38:499-507. [arEM]

Harley, T. A. (1993) Phonological activation of semantic competitors during lexical access in speech production. Language and Cognitive Processes 8:291-309. [aEM]

Heath, M., Neely, K. A., Yakimishyn, J. \& Binsted, G. (2008) Visuomotor memory is independent of conscious awareness of target features. Experimental Brain Research 188:517-27. [aEM]

Hebb, D. O. (1949) The organization of behavior: A neuropsychological theory. Wiley. $[\mathrm{aEM}]$

Helmholtz, H. von (1856/1961) Treatise of physiological optics: Concerning the perceptions in general. In: Classics in psychology, ed. T. Shipley, pp. 79-127. Philosophy Library. (Original work published in 1856). [arEM]

Henis, E. A. \& Flash, T. (1995) Mechanisms underlying the generation of averaged modified trajectories. Biological Cybernetics 72:407-19. [DAR]

Herz, R. S. (2003) The effect of verbal context on olfactory perception. Journal of Experimental Psychology: General 132:595-606. [arEM]

Hesslow, G. (2002) Conscious thought as simulation of behavior and perception. Trends in Cognitive Sciences 6:242-47. [aEM]

Hipwell, A. E., Guo, C., Phillips, M. L., Swain, J. E. \& Moses-Kolko, E. L. (2015) Right frontoinsular cortex and subcortical activity to infant cry is associated with maternal mental state talk. The Journal of Neuroscience 35(37):12725-32. doi: 10.1523/JNEUROSCI.1286-15.2015. [JES]

Hirose, N., Kihara, K., Mima, T., Ueki, Y., Fukuyama, H. \& Osaka, N. (2007) Recovery from object substitution masking induced by transient suppression of visual motion processing: A repetitive transcranial magnetic stimulation study. Journal of Experimental Psychology: Human Perception and Performance 33:1495-503. [PED]

Ho, S. S., Konrath, S., Brown, S. \& Swain, J. E. (2014) Empathy and stress related neural responses in maternal decision making. Frontiers in Neuroscience 8, article 152. (Online journal). doi:10.3389/fnins.2014.00152. [JES]

Ho, V. B., Fitz, C. R., Chuang, S. H. \& Geyer, C. A. (1993) Bilateral basal ganglia lesions: Pediatric differential considerations. RadioGraphics 13:269-92. $[\mathrm{aEM}]$ 
References/Morsella et al.: Homing in on consciousness in the nervous system

Hochberg, J. (1998) Gestalt theory and its legacy: Organization in eye and brain, in attention and mental representation. In: Perception and cognition at century's end: Handbook of perception and cognition, 2nd edition, ed. J. Hochberg, pp. 253-306. Academic Press. [rEM]

Hohwy, J. (2013) The predictive mind. Oxford University Press. [AKS]

Hommel, B. (2009) Action control according to TEC (theory of event coding). Psychological Research 73(4):512-26. [aEM]

Hommel, B. (2010) Grounding attention in action control: The intentional control of selection. In: Effortless attention: A new perspective in the cognitive science of attention and action, ed. B. J. Bruya, pp. 121-40. MIT Press. [EAF]

Hommel, B. (2013) Dancing in the dark: No role for consciousness in action control. Frontiers in Psychology 4, article 380. (Online journal). doi:10.3389/ fpsyg.2013.00380. [arEM, BH]

Hommel, B. (in press) Consciousness and action control. In: Handbook of cognitive control, ed. T. Egner. Wiley. [BH]

Hommel, B. \& Elsner, B. (2009) Acquisition, representation, and control of action. In: Oxford handbook of human action, ed. E. Morsella, J. A. Bargh \& P. M. Gollwitzer, pp. 371-98. Oxford University Press. [aEM]

Hommel, B., Müsseler, J., Aschersleben, G. \& Prinz, W. (2001) The theory of event coding: A framework for perception and action planning. Behavioral and Brain Sciences 24(6):849-37. [aEM]

Hong, L. E., Buchanan, R. W., Thaker, G. K., Shepard, P. D. \& Summerfelt, A. (2008) Beta $(\sim 16 \mathrm{~Hz})$ frequency neural oscillations mediate auditory sensory gating in humans. Psychophysiology 45(2):197-204. [rEM]

Horwitz, B., Amunts, K., Bhattacharyya, R., Patkin, D., Jeffries, K., Zilles, K. \& Braun, A. R. (2003) Activation of Broca's area during the production of spoken and signed language: A combined cytoarchitectonic mapping and PET analysis. Neuropsychologia 41(14):1868-76. [JES]

Hughes, C. M. L. \& Franz, E. A. (2007) Experience-dependent effects in unimanual and bimanual reaction time tasks in musicians. Journal of Motor Behavior 39 (1):3-8. [EAF]

Hughes, G., Velmans, M. \& de Fockert, J. (2009) Unconscious priming of a no-go response. Psychophysiology 46:1258-69. [rEM]

Hughlings Jackson, J. (1884) On affectations of speech from disease of the brain (Part 2). British Medical Journal 12:703-707. [EAF]

Hume, D. (1739/1888) A treatise on human nature, ed. L. A. Selby-Bigge. Oxford University Press. (Original work published in 1739). [aEM]

Hummel, F. \& Gerloff, C. (2005) Larger interregional synchrony is associated with greater behavioral success in a complex sensory integration task in humans. Cerebral Cortex 15:670-78. [arEM]

Hummer, T. A. \& McClintock, M. K. (2009) Putative human pheromone androstadienone attunes the mind specifically to emotional information. Hormones and Behavior 55:548-59. doi:10.1016/j.yhbeh.2009.01.002. [BM]

Humphrey, N. (2006) Seeing red: A study in consciousness. Belknap Press. [DM]

Iacoboni, M. (2005) Understanding others: Imitation, language and empathy. In: Perspectives on imitation: From mirror neurons to memes, ed. S. Hurley \& N. Chater, pp. 77-99. MIT Press. [aEM]

Iacoboni, M. \& Dapretto, M. (2006) The mirror neuron system and the consequences of its dysfunction. Nature Reviews: Neuroscience 7:942-51. [aEM]

Iacoboni, M., Molnar-Szakacs, I., Gallese, V., Buccino, G., Mazziotta, J. C. \& Rizzolatti, G. (2005) Grasping the intentions of others with one's own mirror neuron system. PLoS Biology 3(3):e79. [DB]

Insausti, R., Amaral, D. G. \& Cowan, W. M. (1987) The entorhinal cortex of the monkey: Subcortical afferents. Journal of Comparative Neurology 264:396408. [BM]

Izard, C. E. \& Ackerman, B. P. (2000) Motivational, organizational, and regulatory functions of discrete emotions. In: Handbook of emotions, 2nd edition, ed. M. Lewis \& J. M. Haviland-Jones, pp. 253-65. Guilford Press. [FP]

Jackendoff, R. S. (1990) Consciousness and the computational mind. MIT Press. [arEM]

Jackson, F. (1982) Epiphenomenal qualia. Philosophical Quarterly 32:127-36. [SB]

Jackson, F. (1986) What Mary didn’t know. The Journal of Philosophy 83:291-95. [arEM]

James, W. (1890) The principles of psychology, vols. 1 \& 2. Holt/Dover. [arEM, AV]

James, W. (1890/1983) Principles of psychology. Holt. (Original work published in 1890). [FP]

James, W. (1890/1918) The principles of psychology, vols. 1 and 11. Dover. (Original work published in 1890). [EAF]

Jaśkowski, P. \& Przekoracka-Krawczyk, A. (2005) On the role of mask structure in subliminal priming. Acta Neurobiologiae Experimentalis 65(4):409-17. [AA]

Jaynes, J. (2006) Verbal hallucinations and preconscious mentality. In: Reflections on the dawn of consciousness, ed. M. Kuijsten, pp. 75-94. The Julian Jaynes Society. [DM]

Jeannerod, M. (2006) Motor cognition: What action tells the self. Oxford University Press. [arEM]

Jeannerod, M. (2009) The sense of agency and its disturbances in schizophrenia: A reappraisal. Experimental Brain Research 196:527-32. [aEM]
Johnson, H. \& Haggard, P. (2005) Motor awareness without perceptual awareness. Neuropsychologia 43:227-37. [aEM, BB]

Joliot, M., Ribary, U. \& Llinás, R. (1994) Human oscillatory brain activity near $40 \mathrm{~Hz}$ coexists with cognitive temporal binding. Proceedings of the National Academy of Sciences USA 91:11748-51. [aEM]

Jordan, J. S. (2009) Forward-looking aspects of perception-action coupling as a basis for embodied communication. Discourse Processes 46:127-44. [aEM]

Jordan, J. S. (2013) The wild ways of the conscious will: What we do, how we do it, and why it has meaning. Frontiers in Psychology 4, article 574. (Online journal) doi:10.3389/fpsyg.2013.00574. [JS]]

Juarrero, A. (1999) Dynamics in action: Intentional behavior as a complex system. MIT Press. [CH]

Jung-Beeman, M., Bowden, E. M., Haberman, J., Frymiare, J. L., Arambel-Liu, S., Greenblatt, R., Reber, P. J. \& Kounios, J. (2004) Neural activity when people solve verbal problems with insight. PLoS Biology 2:500-10. [arEM]

Kaido, T., Otsuki, T., Nakama, H., Kaneko, Y., Kubota, Y., Sugai, K. \& Saito, O. (2006) Complex behavioral automatism arising from insular cortex. Epilepsy and Behavior 8:315-19. [aEM]

Kandel, E. R. (2006) In search of memory. W. W. Norton. [DAR]

Kaufman, M. T., Churchland, M. M., Ryu, S. I. \& Shenoy, K. V. (2015) Vacillation, indecision and hesitation in moment-by-moment decoding of monkey cortex. eLife 4:e04677. doi:10.7554/eLife.04677. [rEM]

Kavanagh, D. J., Andrade, J. \& May, J. (2005) Imaginary relish and exquisite torture: The elaborated intrusion theory of desire. Psychological Review 112:446-67. $[\mathrm{BH}]$

Kay, L. M. \& Beshel, J. (2010) A beta oscillation network in the rat olfactory system during a 2-alternative choice odor discrimination task. Journal of Neurophysiology 104:829-39. [rEM]

Kay, L. M., Beshel, J., Brea, J., Martin, C., Rojas-L'bano, D. \& Kopell, N. (2009) Olfactory oscillations: The what, how and what for. Trends in Neurosciences 32:207-14. [rEM]

Kececi, H., Degirmenci, Y. \& Gumus, H. (2013) Two foreign language automatisms in complex partial seizures. Epilepsy and Behavior Case Reports 1:7-9. [aEM]

Keller, A. (2011) Attention and olfactory consciousness. Frontiers in Psychology 2, article 380. (Online journal). doi: 10.3389/fpsyg.2011.00380. [aEM, EAF]

Keller, A. (2014) The evolutionary function of conscious information processing is revealed by its task-dependency in the olfactory system. Frontiers in Psychology 5, article 62. (Online journal). doi:10.3389/fpsyg.2014.00062. [AK]

Kelso, J. A. S., Southard, D. L. \& Goodman, D. (1979) On the nature of human interlimb coordination. Science 203(4384):1029-31. [EAF]

Kern, M. K., Jaradeh, S., Arndorfer, R. C. \& Shaker, R. (2001) Cerebral cortical representation of reflexive and volitional swallowing in humans. American Journal of Physiology: Gastrointestinal and Liver Physiology 280:G354-60. [aEM]

Khan R. M., Luk, C.-H., Flinker, A., Aggarwal, A., Lapid, H., Haddad, R. \& Sobel, N. (2007) Predicting odor pleasantness from odorant structure: Pleasantness as a reflection of the physical world. Journal of Neuroscience 27:10015-23. doi:10.1523/JNEUROSCI.1158-07.2007. [BM]

Kim, J. (2010) Philosophy of mind, 3rd edition. Westview Press. [BLS]

Kim, P., Ho, S. S., Evans, G. W., Liberzon, I. \& Swain, J. E. (2015) Childhood social inequalities influences neural processes in young adult caregiving. Developmental Psychobiology 57(8):948-60. doi: 10.1002/dev.21325. [JES]

Kim, P., Leckman, J. F., Mayes, L. C., Feldman, R., Wang, X. \& Swain, J. E. (2010) The plasticity of human maternal brain: Longitudinal changes in brain anatomy during the early postpartum period. Behavioral Neuroscience 124(5):695700. [JES]

Kim, P., Rigo, P., Mayes, L. C., Feldman, R., Leckman, J. F. \& Swain, J. E. (2014) Neural plasticity in fathers of human infants. Social Neuroscience 9(5):522-35. doi: 10.1080/17470919.2014.933713. [JES]

Kim, P., Strathearn, L. \& Swain, J. E. (2016) The maternal brain and its plasticity in humans. Hormones and Behavior 77:113-23. (Special Issue: Parental Care). doi: 10.1016/j.yhbeh.2015.08.001. [JES]

Kim, S., Singer, B. H. \& Zochowski, M. (2006) Changing roles for temporal representation of odorant during the oscillatory response of the olfactory bulb. Neural Computation 18(4):794-816. [rEM]

Kinsbourne, M. (1996) What qualifies a representation for a role in consciousness? In: Scientific approaches to consciousness, ed. J. D. Cohen \& J. W. Schooler, pp. 335-55. Erlbaum. [arEM]

Kinsbourne, M. (2000) How is consciousness expressed in the cerebral activation manifold? Brain and Mind 2:265-74. [arEM]

Klein, D. B. (1970) A history of scientific psychology: Its origins and philosophical backgrounds. Basic Books. [aEM]

Klein, D. B. (1984) The concept of consciousness: A survey. University of Nebraska Press. [aEM]

Kloos, H. \& Amazeen, E. L. (2002) Perceiving heaviness by dynamic touch: An investigation of the size-weight illusion in preschoolers. British Journal of Developmental Psychology 20(2):171-83. [CH] 
References/Morsella et al.: Homing in on consciousness in the nervous system

Kobal, G., Van Toller, S. \& Hummel, T. (1989) Is there directional smelling? Experientia 45:130-32. [BM]

Kobayakawa, K., Kobayakawa, R., Matsumoto, H., Oka, Y., Imai, T., Ikawa, M., Okabe, M., Ikeda, T., Itohara, S., Kikusui, T., Mori, K. \& Sakano, H. (2007) Innate versus learned odour processing in the mouse olfactory bulb. Nature 450:503-508. doi:10.1038/nature06281. [BM]

Koch, C. (2004) The quest for consciousness: A neurobiological approach. Roberts. [arEM]

Koch, C. \& Tsuchiya, N. (2007) Attention and consciousness: Two distinct brain processes. Trends in Cognitive Sciences 11:16-22. [rEM]

Koch, C. (2012) Consciousness: Confessions of a romantic reductionist. MIT Press. [aEM, PED]

Koch, C. (2014) Consciousness. Colloquium delivered at the Redwood Center for Theoretical Neuroscience, December 2, 2014. University of California. [rEM]

Koch, C. \& Greenfield, S. A. (2007) How does consciousness happen? Scientific American 297:76-83. [aEM]

Koch, C. \& Tononi, G. (2011) A test for consciousness. Scientific American 304 (6):44-47. [LM]

Koch, C. \& Tsuchiya, N. (2007) Attention and consciousness: Two distinct brain processes. Trends in Cognitive Sciences 11:16-22. [PED]

Köhler, W. (1947) Gestalt psychology: An introduction to new concepts in modern psychology. Liveright. [aEM]

Kokkinos, V., Zountsas, B., Kontogiannis, K. \& Garganis, K. (2012) Epileptogenic networks in two patients with hypothalamic hamartoma. Brain Topography 25:327-31. [aEM]

Koriat, A. (2007) Metacognition and consciousness. In: The Cambridge handbook of consciousness, ed. P. D. Zelazo, M. Moscovitch \& E. Thompson, pp. 289-325. Cambridge University Press. [BLS]

Kornell, N. (2014) Where is the "meta" in animal metacognition? Journal of Comparative Psychology 128:143-49. [BLS]

Kosslyn, S. M. (1980) Image and mind. Harvard University Press. [LP]

Kosslyn, S. M., Ganis, G. \& Thompson, W. L. (2001) Neural foundations of imagery. Nature Reviews Neuroscience 2:635-42. [aEM]

Kosslyn, S. M., Thompson, W. L. \& Ganis, G. (2006) The case for mental imagery. Oxford University Press. [aEM]

Köster, E. P. (1971) Adaptation and cross-adaptation in olfaction. Unpublished doctoral dissertation. University of Utrecht. [BM]

Köster, E. P. (2002) The specific characteristics of the sense of smell. In: Olfaction, taste and cognition, ed. C. Rouby, B. Schaal, D. Dubois, R. Gervais \& A. Holley, pp. 27-43. Cambridge University Press. [BM]

Kostic, B., Booth, S. E. \& Cleary, A. M. (2015) The role of analogy in reports of presque vu: Does reporting the presque vu state signal the near retrieval of a source analogy? Journal of Cognitive Psychology 27:739-54. [BLS]

Kouider, S. \& Dupoux, E. (2004) Partial awareness creates the "illusion" of subliminal semantic priming. Psychological Science 15:75-81. [aEM]

Kozhevnikov, M. \& Hegarty, M. (2001) Impetus beliefs as default heuristics: Dissociation between explicit and implicit knowledge about motion. Psychonomic Bulletin and Review 8:439-53. [CH]

Koziol, L., Budding, D. \& Chidekel, D. (2011) From movement to thought: Executive function, embodied cognition, and the cerebellum. The Cerebellum 11:505-25. [JSJ]

Krauzlis, R. J., Bollimunta, A., Arcizet, F. \& Wang, L. (2014) Attention as an effect not a cause. Trends in Cognitive Sciences 18:457-64. [arEM]

Kriegel, U. (2007) A cross-order integration hypothesis for the neural correlate of consciousness. Consciousness and Cognition 16:897-912. [aEM]

Krist, H., Fieberg, E. L. \& Wilkening, F. (1993) Intuitive physics in action and judgment: The development of knowledge about projectile motion. Journal of Experimental Psychology: Learning, Memory and Cognition 19:952-66. $[\mathrm{CH}]$

Kutlu, G., Bilir, E., Erdem, A., Gomceli, Y. B., Kurt, G. S. \& Serdaroglu, A. (2005) Hush sign: A new clinical sign in temporal lobe epilepsy. Epilepsy and Behavior 6:452-55. [aEM]

Laing, D. G. (1983) Natural sniffing gives optimum odour perception for humans. Perception 12:99-117. [EAF]

Lamme, V. A. (2001) Blindsight: The role of feedback and feedforward cortical connections. Acta Psychologica 107:209-28. [aEM]

Lamme, V. A. F. \& Roelfsema, P. R. (2000) The distinct modes of vision offered by feedforward and recurrent processing. Trends in Neuroscience 23(11):57179. [LM]

Lamme, V. A. F. \& Spekreijse, H. (2000) Modulations of primary visual cortex activity representing attentive and conscious scene perception. Frontiers in Bioscience 5:D232-43. [aEM]

Långsjö, J. W., Alkire, M. T., Kaskinoro, K., Hayama, H., Maksimow, A., Kaisti, K. K., Aalto, S., Aantaa, R., Jääskeläinen, S. K., Revonsuo, A. \& Scheinin, H. (2012) Returning from oblivion: Imaging the neural core of consciousness. The Journal of Neuroscience 32:4935-43. [arEM]
Lashley, K. S. (1942) The problem of cerebral organization in vision. In: Visual mechanisms: Biological symposia, vol. 7, ed. H. Kluver, pp. 301-22. Cattell Press. [aEM]

Lashley, K. S. (1951) The problem of serial order in behavior. In: Cerebral mechanisms in behavior: The Hixon symposium, ed. L. A. Jeffress, pp. 112-46. Wiley. [aEM]

Lashley, K. S. (1956) Cerebral organization and behavior. Proceedings of the Association for Research in Nervous and Mental Diseases 36:1-18. [arEM]

Lathe, R. (2001) Hormones and the hippocampus. Journal of Endocrinology 169:205-31. [RL]

Lau, H. C. (2008) A higher-order Bayesian decision theory of consciousness. Progress in Brain Research 168:35-48. [aEM]

Lau, H. C. (2009) Volition and the functions of consciousness. In: The Cognitive Neurosciences IV, ed. M. Gazzaniga, pp. 1191-200. MIT Press. [aEM]

Lau, H. C., Rogers, R. D. \& Passingham, R. E. (2007) Manipulating the experienced onset of intention after action execution. Journal of Cognitive Neuroscience 19:81-90. [aEM]

Laurent, G. \& Davidowitz, H. (1994) Encoding of olfactory information with oscillating neural assemblies. Science 265(5180):1872-75. [rEM]

Laureys, S. (2005) The neural correlate of (un)awareness: Lessons from the vegetative state. Trends in Cognitive Sciences 12:556-59. [aEM]

Lawless, H. T. (1997) Olfactory psychophysics. In: Tasting and Smelling: Handbook of Perception and Cognition, ed. G. K. Beauchamp \& L. Bartoshuk, pp. 125-74. Academic Press. [aEM]

LeDoux, J. E. (1996) The emotional brain: The mysterious underpinnings of emotional life. Simon and Schuster. [arEM]

LeDoux, J. E. (2000) Emotion circuits in the brain. Annual Review of Neuroscience 23:155-84. [rEM]

LeDoux, J. E. (2012) Rethinking the emotional brain. Neuron 73:653-76. [rEM]

Lee, U., Kim, S., Noh, G. J., Choi, B. M., Hwang, E. \& Mashour, G. (2009) The directionality and functional organization of frontoparietal connectivity during consciousness and anesthesia in humans. Consciousness and Cognition 18:1069-78. [aEM]

Lee, U., Kim, S., Noh, G. J., Choi, B. M., Hwang, E. \& Mashour, G. (2009) The directionality and functional organization of frontoparietal connectivity during consciousness and anesthesia in humans. Consciousness and Cognition 18:1069-78. [rEM]

Lehar, S. (2003) Gestalt isomorphism and the primacy of the subjective conscious experience: A Gestalt bubble model. Behavioral and Brain Sciences 26:37544. [aEM]

Lenzi, D., Trentini, C., Pantano, P., Macaluso, E., Iacoboni, M., Lenzi, G. L. \& Ammaniti, M. (2009) Neural basis of maternal communication and emotional expression processing during infant preverbal stage. Cerebral Cortex 19 (5):1124-33. doi: 10.1093/cercor/bhn153. [JES]

Levelt, W. J. M. (1989) Speaking: From intention to articulation. MIT Press. [arEM]

Leventhal, H. (1987) A perceptual motor theory of emotion. In: Advances in experimental social psychology, vol. 17, ed. L. Berkowitz, pp. 117-82. Academic Press. [GG]

Levine, D. S. (2012) I think therefore I feel: Possible neural mechanisms for knowledge-based pleasure. In: Proceedings of the 2012 International Joint Conference on Neural Networks (IJCNN), Brisbane, Australia, June 10-15, 2012, pp. 1-5. IEEE Press. doi: 10.1109/IJCNN.2012.6252413. [LP]

Levitin, D. J. (2014) The organized mind: Thinking straight in the age of information overload. Dutton. [BH]

Lewin, K. (1935) A dynamic theory of personality. McGraw-Hill. [aEM]

Lewis, L. D., Weiner, V. S., Mukamel, E. A., Donoghue, J. A., Eskandar, E. N., Madsen, J. R., Anderson, W., Hochberg, L. R., Cash, S. S., Brown, E. N. \& Purdon, P. L. (2012) Rapid fragmentation of neuronal networks at the onset of propofol-induced unconsciousness. Proceedings of the National Academy of Sciences USA 109(49):E3377-86. [arEM]

Lhermitte, F. (1983) "Utilization behavior" and its relation to lesions of the frontal lobes. Brain 106:237-55. [aEM]

Li, W., Lopez, L., Osher, J., Howard, J. D., Parrish, T. B. \& Gottfried, J. A. (2010) Right orbitofrontal cortex mediates conscious olfactory perception. Psychological Science 21:1454-63. [aEM]

Libet, B. (2004) Mind time: The temporal factor in consciousness. Harvard University Press. [aEM]

Lieberman, M. D. (2007) The X- and C-systems: The neural basis of automatic and controlled social cognition. In: Fundamentals of social neuroscience, ed. E. Harmon-Jones \& P. Winkielman, pp. 290-315. Guilford. [aEM]

Lin, Z. \& He, S. (2009) Seeing the invisible: The scope and limits of unconscious processing in binocular rivalry. Progress in Neurobiology 87(4):195-211. [ZL]

Lin, Z. \& Murray, S. O. (2013) Visible propagation from invisible exogenous cueing. Journal of Vision 13(11):1-15. [ZL]

Lin, Z. \& Murray, S. O. (2014a) Priming of awareness or how not to measure visual awareness. Journal of Vision 14(1):1-17. [ZL]

Lin, Z. \& Murray, S. O. (2014b) Unconscious processing of an abstract concept. Psychological Science 25(1):296-98. [ZL] 
Lin, Z. \& Murray, S. O. (2015a) Automaticity of unconscious response inhibition: Comment on Chiu and Aron (2014) Journal of Experimental Psychology: General 144(1):244-54. [ZL]

Lin, Z. \& Murray, S. O. (2015b) More power to the unconscious: Conscious, but not unconscious, exogenous attention requires location variation. Psychological Science 26(2):221-30. [ZL]

Liu, C. H., Tzeng, O. J., Hung, D. L., Tseng, P. \& Juan, C. H. (2012) Investigation of bistable perception with the "silhouette spinner": Sit still, spin the dancer with your will. Vision Research 60:34-39. [DB]

Liu, G., Chua, R. \& Enns, J. T. (2008) Attention for perception and action: Task interference for action planning, but not for online control. Experimental Brain Research 185:709-17. [aEM]

Liu, Y., Paradis, A.-L., Yahia-Cherif, L. \& Tallon-Baudry, C. (2012) Activity in the lateral occipital cortex between 200 and $300 \mathrm{~ms}$ distinguishes between physically identical seen and unseen stimuli. Frontiers in Human Neuroscience 6, article 211. (Online journal). [aEM]

Livnat, A. \& Pippenger, N. (2006) An optimal brain can be composed of conflicting agents. Proceedings of the National Academy of Sciences USA 103:3198-202. [rEM]

Llinás, R. R. \& Ribary, U. (2001) Consciousness and the brain: The thalamocortical dialogue in health and disease. Annals of the New York Academy of Sciences 929:166-75. [aEM]

Llinás, R., Ribary, U., Contreras, D. \& Pedroarena, C. (1998) The neuronal basis for consciousness. Philosophical Transactions of the Royal Society of London: B 353:1841-49. [aEM]

Loewenstein, G. (1996) Out of control: visceral influences on behavior. Organizational Behavior and Human Decision Processes 65:272-92. [rEM]

Logan, G. D., Taylor, S. E. \& Etherton, J. L. (1999) Attention and automaticity: Toward a theoretical integration. Psychological Research 62:165-81. [rEM]

Logan, G. D., Yamaguchi, M., Schall, J. D. \& Palmeri, T. J. (2015) Inhibitory control in mind and brain 2.0: Blocked-input models of saccadic countermanding. Psychological Review 122:115-47. [arEM]

Logothetis, N. K. \& Schall, J. D. (1989) Neuronal correlates of subjective visual perception. Science 245:761-62. [arEM]

Lorenz, K. (1963) On aggression. Harcourt, Brace \& World. [arEM]

Lotze, R. H. (1852) Medizinische Psychologie oder Physiologie der Seele. Weidmann'sche Buchhandlung. [aEM]

Lucas, M. (2000) Semantic priming without association: A meta-analytic review. Psychonomic Bulletin and Review 7:618-30. [aEM]

Lundström, J. N., Boyle, J. A., Zatorre, J. C. \& Jones-Gotman, M. (2008) Functional neuronal processing of body odors differs from that of similar common odors. Cerebral Cortex 18:1466-74. doi:10.1093/cercor/bhm178. [BM]

Lundström, J. N. \& Olsson, M. J. (2005) Subthreshold amounts of social odorant affect mood, but not behavior, in heterosexual women when tested by a male, but not a female, experimenter. Biological Psychology 70:197-204. [BM]

MacGregor, J. N. \& Ormerod, T. (1996) Human performance on the traveling salesman problem. Perception and Psychophysics 58(4):527-39. [DB]

Mack, A. \& Rock, I. (1998) Inattentional blindness. MIT Press. [PED]

MacLeod, C. M. \& MacDonald, P. A. (2000) Interdimensional interference in the Stroop effect: Uncovering the cognitive and neural anatomy of attention. Trends in Cognitive Sciences 4:383-91. [rEM]

Macphail, E. M. (1998) The evolution of consciousness. Oxford University Press. [arEM]

Mainland, J. D. \& Sobel, N. (2006) The sniff is part of the olfactory percept. Chemical Senses 31:181-96. [arEM, EAF]

Mak, Y. E., Simmons, K. B., Gitelman, D. R. \& Small, D. M. (2005) Taste and olfactory intensity perception changes following left insular stroke. Behavioral Neuroscience 119:1693-700. [aEM]

Mandairon, N., Poncelet, J., Bensafi, M. \& Didier A. (2009) Humans and mice express similar olfactory preferences. PLoS ONE 4(1):e4209. doi:10.1371/ journal.pone.0004209. $\quad[\mathrm{BM}]$

Marcel, A. J. (1993) Slippage in the unity of consciousness. In: Experimental and theoretical studies of consciousness. Ciba Foundation Symposium 174, ed. G. R. Bock \& J. Marsh, pp. 168-80. Wiley. [rEM]

Marchetti, C. \& Della Sala, S. (1998) Disentangling the alien and anarchic hand. Cognitive Neuropsychiatry 3:191-207. [aEM]

Marcus, G. (2008) Kluge: The haphazard construction of the mind. Houghton Mifflin. [aEM]

Mareschal, D., Johnson, M. H., Sirois, S., Spratling, M. W., Thomas, M. C. S. \& Westermann, G. (2007) Neuroconstructivism, vol. 1: How the brain constructs cognition. Oxford University Press. [HD]

Maril, A., Simons, J. S., Weaver, J. J. \& Schacter, D. L. (2005) Graded recall success: An event-related fMRI comparison of tip of the tongue and feeling of knowing Neuroimage 24:1130-38. [BLS]

Maril, A., Wagner, A. D. \& Schacter, D. L. (2001) On the tip of the tongue: An event-related fMRI study of semantic retrieval failure and cognitive conflict. Neuron 31:653-60. [BLS]

Markman, A. B. (1999) Knowledge representation. Erlbaum. [aEM]
Markowitsch, H. J. (1982) Thalamic mediodorsal nucleus and memory: A critical evaluation of studies in animals and man. Neuroscience and Biobehavioral Reviews 6:351-80. [aEM]

Marois, R., Yi, D. J. \& Chun, M. M. (2004) The neural fate of perceived and missed events in the attentional blink. Neuron 41:465-72. [PED]

Marr, D. (1982) Vision: A computational investigation into the human representation and processing of visual information. W. H. Freeman. [ $[\mathrm{rEM}]$

Martin, C., Beshel, J. \& Kay, L. M. (2007) An olfacto-hippocampal network is dynamically involved in odor-discrimination learning. Journal of Neurophysiology 98(4):2196-205. [rEM]

Martin, G. N. (2013) The neuropsychology of smell and taste. Psychology Press. [aEM]

Masataka, N. \& Perlovsky, L. I. (2012) The efficacy of musical emotions provoked by Mozart's music for the reconciliation of cognitive dissonance. Scientific Reports 2, Article number: 694. (Online publication). doi:10.1038/srep00694. [LP]

Mashour, G. A. (2004) Consciousness unbound: Toward a paradigm of general anesthesia. Anesthesiology 100:428-33. [rEM]

Masicampo, E. J. \& Baumeister, R. F. (2013) Conscious thought does not guide momentto-moment actions - it serves social and cultural functions. Frontiers in Psychology 4, article 478. (Online journal). doi:10.3389/fpsyg.2013.00478. [arEM]

Massaro, D. W. (1998) Perceiving talking faces: From speech perception to a behavioral principle. MIT Press. [DM]

Mast, T. G. \& Samuelsen, C. L. (2009) Human pheromone detection by the vomeronasal organ: Unnecessary for mate selection? Chemical Senses 34:529-31. doi:10.1093/chemse/bjp030. [BM]

Mathalon, D. H. \& Ford, J. M. (2008) Corollary discharge dysfunction in schizophrenia: Evidence for an elemental deficit. Clinical EEG and Neuroscience 39:82-86. [aEM]

Mattler, U. (2005) Flanker effects on motor output and the late-level response activation hypothesis. Quarterly Journal of Experimental Psychology 58A:577601. [aEM]

Mayr, E. (2001) What evolution is. Weidenfeld \& Nicolson. [rEM]

McClelland, J. L. (1979) On the time-relations of mental processes: An examination of systems of processes in cascade. Psychological Review 86:287-30. [arEM]

McFarland, D. J. \& Sibly, R. M. (1975) The behavioural final common path. Philosophical Transactions of the Royal Society (London) 270:265-93. [aEM]

McGurk, H. \& MacDonald, J. (1976) Hearing lips and seeing voices. Nature 264:746-48. [aEM]

McKay, L. C., Evans, K. C., Frackowiak, R. S. J. \& Corfield, D. R. (2003) Neural correlates of voluntary breathing in humans determined using functional magnetic resonance imaging. Journal of Applied Physiology 95:1170-78. [aEM]

McClintock, M. K. (2000) Human pheromones: Primers, releasers, signalers, or modulators? In: Reproduction in context, ed. K. Wallen \& J. E. Schneider, pp. 355-420. MIT Press. [BM]

Meador, K. J., Ray, P. G., Echauz, J. R., Loring, D. W. \& Vachtsevanos, G. J. (2002) Gamma coherence and conscious perception. Neurology 59:847-54. [arEM]

Meier, B. P., Robinson, M. D., Crawford, L. E. \& Ahlvers, W. J. (2007) When "light" and "dark" thoughts become light and dark responses: Affect biases brightness judgments. Emotion 7:366-76. [aEM]

Melcher, T., Weidema, M., Eenshuistra, R. M., Hommel, B. \& Gruber, O. (2008) The neural substrate of the ideomotor principle: An event-related fMRI analysis. NeuroImage 39:1274-88. [aEM]

Melcher, T., Winter, D., Hommel, B., Pfister, R., Dechent, P. \& Gruber, O. (2013) The neural substrate of the ideomotor principle revisited: Evidence for asymmetries in action-effect learning. Neuroscience 231:13-27. [aEM]

Melzack, R. \& Casey, K. L. (1968) Sensory, motivational, and central control determinants of pain: A new conceptual model. In: The skin senses, ed. D. R. Kenshalo, pp. 423-39. Charles C. Thomas. [rEM]

Mendl, M. \& Paul, E. S. (2004) Consciousness, emotion and animal welfare: Insights from cognitive science. Animal Welfare 13(Suppl.):S17-25. [AV]

Merker, B. (2005) The liabilities of mobility: A selection pressure for the transition to consciousness in animal evolution. Consciousness and Cognition 14(1):89-114. doi: 10.1016/S1053-8100(03)00002-3. [AKS]

Merker, B. (2007) Consciousness without a cerebral cortex: A challenge for neuroscience and medicine. Behavioral and Brain Sciences 30(1):63-81; discussion 81-134. [arEM, BM]

Merker, B. (2012) From probabilities to percepts: A subcortical "global best estimate buffer" as locus of phenomenal experience. In: Being in time: Dynamical models of phenomenal experience, ed. E. Shimon, F. Tomer \& N. Zach, pp. 37-80. John Benjamins. [arEM, BM]

Merker, B. (2013a) Body and world as phenomenal contents of the brain's reality model. In: The unity of mind, brain and world: Current perspectives on a science of consciousness, ed. A. Pereira, Jr. \& D. Lehmann, pp. 7-42. Cambridge University Press. [BM]

Merker, B. (2013b) Cortical gamma oscillations: The functional key is activation, not cognition. Neuroscience and Biobehavioral Reviews 37:401-17. [aEM]

Merker, B. (2013c) The efference cascade, consciousness, and its self: Naturalizing the first person pivot of action control. Frontiers in Psychology 4, article 501:120. (Online journal). doi:10.3389/fpsyg.2013.00501. [arEM] 
References/Morsella et al.: Homing in on consciousness in the nervous system

Merrick, C., Farnia, M., Jantz, T. K., Gazzaley, A. \& Morsella, E. (2015) External control of the stream of consciousness: Stimulus-based effects on involuntary thought sequences. Consciousness and Cognition 33:217-225. [rEM]

Merrick, M. C., Godwin, C. A., Geisler, M. W. \& Morsella, E. (2014) The olfactory system as the gateway to the neural correlates of consciousness. Frontiers in Psychology 4, article1011. (Online journal). doi:10.3389/fpsyg.2013.01011. [arEM, RL]

Metcalfe, J. \& Mischel, W. (1999) A hot/cool-system analysis of delay of gratification: Dynamics of willpower. Psychological Review 106:3-19. [aEM]

Metcalfe, J. \& Schwartz, B. L. (2016) The ghost in the machine: Self-reflective consciousness and the neuroscience of metacognition. In: Oxford handbook of metamemory, ed. J. Dunlosky \& S. Tauber, pp. 407-24. Oxford University Press. [BLS]

Metzinger, T. (2000) Neural correlates of consciousness. MIT Press. [aEM]

Miall, R. C. (2003) Connecting mirror neuron and forward models. Neuroreport 14:1-3. [aEM]

Miller, E. K. (2000) The prefrontal cortex and cognitive control. Nature Reviews Neuroscience 1:59-65. [aEM]

Miller, G. A. (1962) Psychology: The science of mental life. Adams, Bannister, \& Cox. [aEM]

Miller, N. E. (1959) Liberalization of basic S-R concepts: Extensions to conflict behavior, motivation, and social learning. In: Psychology: A study of a science, vol. 2, ed. S. Koch, pp. 196-292. McGraw-Hill. [arEM]

Milner, A. D. \& Goodale, M. (1995) The visual brain in action. Oxford University Press. [aEM]

Milner, B. (1966) Amnesia following operation on the temporal lobes. In: Amnesia, ed. C. W. M. Whitty \& O. L. Zangwill, pp. 109-33. Butterworths. [aEM]

Mink, J. W. (1996) The basal ganglia: Focused selection and inhibition of competing motor programs. Progress in Neurobiology 50:381-425. [EAF]

Minsky, M. (1985) The society of mind. Simon and Schuster. [rEM]

Mitchell, A. S., Baxter, M. G. \& Gaffan, D. (2007) Dissociable performance on scene learning and strategy implementation after lesions to magnocellular mediodorsal thalamic nucleus. The Journal of Neuroscience 27:11888-95. [aEM]

Mizobuchi, M., Ito, N., Tanaka, C., Sako, K., Sumi, Y. \& Sasaki, T. (1999) Unidirectional olfactory hallucination associated with ipsilateral unruptured intracranial aneurysm. Epilepsia 40:516-19. [aEM]

Molapour, T., Berger, C. C. \& Morsella, E. (2011) Did I read or did I name? Process blindness from congruent processing "outputs." Consciousness and Cognition 20:1776-80. [arEM]

Mori, K. \& Sakano, H. (2011) How is the olfactory map formed and interpreted in the mammalian brain? Annual Review of Neuroscience 34:467-99. doi:10.1146/ annurev-neuro-112210-112917. [BM]

Morsella, E. (2005) The function of phenomenal states: Supramodular interaction theory. Psychological Review 112:1000-21. [arEM, AK, BM]

Morsella, E. \& Bargh, J. A. (2007) Supracortical consciousness: Insights from temporal dynamics, processing-content, and olfaction. Behavioral and Brain Sciences 30:100. [aEM]

Morsella, E. \& Bargh, J. A. (2010a) Unconscious mind. In: The Corsini encyclopedia of psychology and behavioral science, vol. 4, 4th edition, ed. I. B. Weiner \& W. E. Craighead, pp. 1817-19. Wiley. [aEM]

Morsella, E. \& Bargh, J. A. (2010b) What is an output? Psychological Inquiry 21:354-70. [arEM]

Morsella, E. \& Bargh, J. A. (2011) Unconscious action tendencies: Sources of "unintegrated" action. In: The handbook of social neuroscience, ed. J. T. Cacioppo \& J. Decety, pp. 335-47. Oxford University Press. [arEM]

Morsella, E., Berger, C. C. \& Krieger, S. C. (2011) Cognitive and neural components of the phenomenology of agency. Neurocase 17:209-30. [arEM]

Morsella, E., Gray, J. R., Krieger, S. C. \& Bargh, J. A. (2009a) The essence of conscious conflict: Subjective effects of sustaining incompatible intentions. Emotion 9:717-28. [arEM]

Morsella, E., Krieger, S. C. \& Bargh, J. A. (2010) Minimal neuroanatomy for a conscious brain: Homing in on the networks constituting consciousness. Neural Networks 23:14-15. [aEM]

Morsella, E., Lanska, M., Berger, C. C. \& Gazzaley, A. (2009b) Indirect cognitive control through top-down activation of perceptual symbols. European Journal of Social Psychology 39:1173-77. [aEM]

Morsella, E. \& Poehlman, T. A. (2013) The inevitable contrast: Conscious versus unconscious processes in action control. Frontiers in Psychology 4, article 590. (Online journal). doi:10.3889/fpsyg.2013.00590. [aEM]

Morsella, E., Wilson, L. E., Berger, C. C., Honhongva, M., Gazzaley, A. \& Bargh, J. A. (2009c) Subjective aspects of cognitive control at different stages of processing. Attention, Perception and Psychophysics 71:1807-24. [arEM]

Morsella, E., Zarolia, P. \& Gazzaley, A. (2012) Cognitive conflict and consciousness. In: Cognitive consistency: A unifying concept in social psychology, ed. B. Gawronski \& F. Strack, pp. 19-46. Guilford Press. [rEM]

Moses-Kolko, E. L., Horner, M. S., Phillips, M. L., Hipwell, A. E. \& Swain, J. E. (2014) In search of neural endophenotypes of postpartum psychopathology and disrupted maternal caregiving. Journal of Neuroendocrinology 26(10):665-84. doi: 10.1111/jne.12183. [JES]

Mudrik, L., Breska, A., Lamy, D. \& Deouell, L. Y. (2011) Integration without awareness: Expanding the limits of unconscious processing. Psychological Science 22(6):764-70. [ZL]

Mudrik, L., Faivre, N. \& Koch, C. (2014) Information integration without awareness. Trends in Cognitive Sciences 18(9):488-96. [arEM, LM]

Mujica-Parodi, L. R., Strey, H. H., Frederick, B., Savoy, R., Cox, D., Botanov, Y., Tolkunov, D., Rubin, D. \& Weber, J. (2009) Chemosensory cues to conspecific emotional stress activate amygdala in humans. PLoS ONE 4(7):e6415. doi:10.1371/journal.pone.0006415. [BM]

Müller, J. (1843) Elements of physiology. Lea and Blanchard. [aEM]

Munakata, Y., Herd, S. A., Chatham, C. H., Depue, B. E., Banich, M. T. \& O’Reilly, R. C. (2011) A unified framework for inhibitory control. Trends in Cognitive Sciences 15:453-59. [rEM]

Muzur, A., Pace-Schott, E. F. \& Hobson, J. A. (2002) The prefrontal cortex in sleep. Trends in Cognitive Sciences 6:475-81. [aEM]

Nachev, P., Kennard, C. \& Husain, M. (2008) Functional role of the supplementary and pre-supplementary motor areas. Nature Reviews: Neuroscience 9(11):85669. doi: 10.1038/nrn2478. [JES]

Nagasako, E. M., Oaklander, A. L. \& Dworkin, R. H. (2003) Congenital insensitivity to pain: An update. Pain 101:213-19. [rEM]

Nagel, T. (1974) What is it like to be a bat? Philosophical Review 83:435-50. [rEM]

Nath, A. R. \& Beauchamp, M. S. (2012) A neural basis for interindividual differences in the McGurk effect, a multisensory speech illusion. NeuroImage 59:781-87. [rEM]

Neumann, O. (1987) Beyond capacity: A functional view of attention. In: Perspectives on perception and action, ed. H. Heuer \& A. F. Sanders, pp. 361-94. Erlbaum. [rEM]

Neville, K. R. \& Haberly, L. B. (2003) Beta and gamma oscillations in the olfactory system of the urethane-anesthetized rat. Journal of Neurophysiology 90 (6):3921-30. [rEM]

Nieuwenhuys, R., Ten Donkelaar, H. J. \& Nicholson, C. (1997) The central nervous system of vertebrates. Springer. [RL]

Nisbett, R. E. \& Wilson, T. D. (1977) Telling more than we can know: Verbal reports on mental processes. Psychological Review 84:231-59. [rEM]

Nishizumi, H. \& Sakano, H. (2015) Decoding and deorphanizing an olfactory map. Nature Neuroscience 18:1432-33. [BM]

Norman, D. (2013) The design of everyday things. (Revised and expanded edition.) Basic Books. [JdV]

Oatley, K. \& Johnson-Laird, P. (1987) Toward a cognitive theory of emotions. Cognition and Emotion 1:29-50. [GG]

Oberauer, K. \& Hein, L. (2012) Attention to information in working memory. Current Directions in Psychological Science 21:164-69. [ [rEM]

Obhi, S. S. \& Haggard, P. (2004) Free will and free won't. American Scientist 92:358-65. [DM]

Obhi, S., Planetta, P. \& Scantlebury, J. (2009) On the signals underlying conscious awareness of action. Cognition 110:65-73. [aEM]

Öhman, A., Carlsson, K., Lundqvist, D. \& Ingvar, M. (2007) On the unconscious subcortical origin of human fear. Physiology and Behavior 92:180-85. [aEM]

Öhman, A. \& Mineka, S. (2001) Fears, phobias, and preparedness: Toward an evolved module of fear and fear learning. Psychological Review 108:483-522. [arEM]

Ojemann, G. (1986) Brain mechanisms for consciousness and conscious experience. Canadian Psychology 27:158-68. [aEM]

Olsson, A. \& Phelps, E. A. (2004) Learned fear of "unseen" faces after Pavlovian, observational, and instructed fear. Psychological Science 15:822-28. [arEM]

Ortinski, P. \& Meador, K. J. (2004) Neuronal mechanisms of conscious awareness. Archives of Neurology 61:1017-20. [aEM]

O'Shea, R. P. \& Corballis, P. M. (2005) Visual grouping on binocular rivalry in a splitbrain observer. Vision Research 45:247-61. [aEM]

Pacherie, E. (2008) The phenomenology of action: A conceptual framework. Cognition 107:179-217. [aEM]

Pahlavan, F. \& Arouss, M. (2015) Executive regulation of anxiety and aggression. Journal of Behavioral and Brain Science, Special Issue on "Emotion, Cognition, and Behavior." 6:53-80. doi: http://dx.doi.org/10.1017/S0140525X15000643 [FP]

Pahlavan, F., Bonnet, P. \& Duda, D. (2000a) Human motor responses to simultaneous aversive stimulation and failure on a valued task. Psychological Reports 86:232-42. [FP]

Pahlavan, F., Duda, D. \& Bonnet, P. (2000b) Direction of human motor responses by men and women to aversive stimulation. Psychological Reports: Perceptual and Motor Skills 90:415-22. [FP]

Pahlavan, F. \& Lubart, T. (2005) Negative affective states' effects on perception of affective pictures. Consciousness and Emotion 1:57-79. [FP]

Pahlavan, F. \& Lubart, T. (2007) Motivational effects of success versus failure feedback. Psychological Reports 100:585-603. [FP] 
References/Morsella et al.: Homing in on consciousness in the nervous system

Pahlavan, F., Mouchiroud, C. \& Nemlaghi-Manis, E. (2012) Does experience of failure decrease executive, regulatory abilities and increase aggression? Global Journal of Health Science 4(6):60-87. [FP]

Panagiotaropolous, T. I., Deco, G., Kapoor, V. \& Logothetis, N. K. (2012) Neuronal discharges and gamma oscillations explicitly reflect visual consciousness in the lateral prefrontal cortex. Neuron 74:924-35. [arEM]

Panagiotaropoulos, T. I., Kapoor, V. \& Logothetis, N. K. (2013) Desynchronization and rebound of beta oscillations during conscious and unconscious local neuronal processing in the macaque lateral prefrontal cortex. Frontiers in Psychology 4, article 603. (Online journal). doi:10.3389/fpsyg.2013.00603. [aEM]

Panksepp, J. (1998) Affective neuroscience: The foundations of human and animal emotions. Oxford University Press. [aEM]

Panksepp, J. (2005) Affective consciousness: Core emotional feelings in animals and humans. Consciousness and Cognition 14:30-80. [AV]

Panksepp, J. (2007) Emotional feelings originate below the neocortex: Toward a biology of the soul. Behavioral and Brain Sciences 30:101-103. [aEM]

Parkinson, J. \& Haggard, P. (2014) Subliminal priming of intentional inhibition. Cognition 130(2):255-65. doi: 10.1016/j.cognition.2013.11.005. [AKS]

Pascual-Leone, A. \& Walsh, V. (2001) Fast backprojections from the motion to the primary visual area are necessary for visual awareness. Science 292:510-12. [aEM]

Pashler, H. (1993) Doing two things at the same time. American Scientist 81:48-55. [DAR, rEM]

Passingham, R. (1995) The frontal lobes and voluntary action. Oxford University Press. [rEM]

Penfield, W. \& Jasper, H. H. (1954) Epilepsy and the functional anatomy of the human brain. Little, Brown. [aEM]

Penfield, W. \& Roberts, L. (1959) Speech and brain mechanisms. Princeton University Press. [aEM]

Perlovsky, L. I. (1998) Conundrum of combinatorial complexity. IEEE Transactions on Pattern Analysis and Machine Intelligence 20(6):666-70. [LP]

Perlovsky, L. I. (2001) Neural networks and intellect: Using model-based concepts. Oxford University Press. [LP]

Perlovsky, L. I. (2006) Toward physics of the mind: Concepts, emotions, consciousness, and symbols. Physics of Life Reviews 3(1):22-55. [LP]

Perlovsky, L. I. (2007) Neural dynamic logic of consciousness: The knowledge instinct. In: Neurodynamics of higher-level cognition and consciousness, ed. L. I. Perlovsky \& R. Kozma, pp. 73-108. Springer-Verlag. [LP]

Perlovsky, L. I. (2009) "Vague-to-crisp" neural mechanism of perception. IEEE Transactions on Neural Networks 20(8):1363-67. [LP]

Perlovsky, L. I. (2010a) Intersections of mathematical, cognitive, and aesthetic theories of mind. Psychology of Aesthetics, Creativity, and the Arts 4(1):11-17. doi: 10.1037/a0018147. [LP]

Perlovsky, L.I. (2010b) Musical emotions: Functions, origin, evolution. Physics of Life Reviews 7(1):2-27. doi:10.1016/j.plrev.2009.11.001. [LP]

Perlovsky, L. I. (2012a) Cognitive function of music: Part I. Interdisciplinary Science Reviews 37(2):129-42. [LP]

Perlovsky, L. I. (2012b) Cognitive function, origin, and evolution of musical emotions. Musicae Scientiae 16(2):185-99. doi: 10.1177/1029864912448327. [LP]

Perlovsky, L. I. (2013a) A cognitive model of language and conscious processes. In: The unity of mind, brain and world, ed. A. Pereira, Jr. \& D. Lehmann, pp. 26568. Cambridge University Press. [LP]

Perlovsky, L. I. (2013b) Language and cognition - joint acquisition, dual hierarchy, and emotional prosody. Frontiers in Behavioral Neuroscience 7, article 123. (Online journal). doi:10.3389/fnbeh.2013. 00123. [LP]

Perlovsky, L. I. (2013c) Learning in brain and machine - complexity, Gödel, Aristotle. Frontiers in Neurorobotics 7, article23. (Online journal). doi:10.3389/ fnbot.2013.00023. [LP]

Perlovsky, L. I. (2014a) Aesthetic emotions, what are their cognitive functions? Frontiers in Psychology 5, article 98. (Online journal) doi:10.3389/ fpsyg.2014.00098. Available at: http://www.frontiersin.org/Journal/10.3389/ fpsyg.2014.00098/full. [LP]

Perlovsky, L. I. (2014b) The cognitive function of music: Part II. Interdisciplinary Science Reviews 39(2):162-86. [LP]

Perlovsky, L. I. (2015) Origin of music and the embodied cognition. Frontiers in Psychology 6, article 538. (Online publication). Available at: http://dx.doi.org/ 10.3389/fpsyg.2015.00538. [LP]

Perlovsky, L. I., Bonniot-Cabanac, M.-C. \& Cabanac, M. (2010) Curiosity and pleasure. Webmed Central Psychology 1(12):WMC001275. Available at: http:// www.webmedcentral.com/ article_view/1275 [LP]

Perlovsky, L. I., Cabanac, A., Bonniot-Cabanac, M.-C. \& Cabanac, M. (2013) Mozart effect, cognitive dissonance, and the pleasure of music. Behavioural Brain Research 244:9-14. [LP]

Philippi, C. L., Feinstein, J. S., Khalsa, S. S., Damasio, A., Tranel, D., Landini, G. Williford, K. \& Rudrauf, D. (2012) Preserved self-awareness following extensive bilateral brain damage to the insula, anterior cingulate, and medial prefrontal cortices. PLoS ONE 7:e38413. [aEM]
Pilon, M. \& Sullivan, S. J. (1996) Motor profile of patients in minimally responsive and persistent vegetative states. Brain Injury 10:421-37. [aEM]

Pinker, S. (1997) How the mind works. Norton. [arEM]

Plailly, J., Howard, J. D., Gitelman, D. R. \& Gottfried, J. A. (2008) Attention to odor modulates thalamocortical connectivity in the human brain. Journal of Neuroscience 28:5257-67. doi:10.1523/JNEUROSCI.5607-07.2008. [aEM, BM]

Poellinger, A., Thomas, R., Lio, P., Lee, A., Makris, N., Rosen, B. R. \& Kwong, K. K. (2001) Activation and habituation in olfaction-an fMRI study. Neuroimage 13 (4):547-60. [rEM]

Poehlman, T. A., Jantz, T. K. \& Morsella, E. (2012) Adaptive skeletal muscle action requires anticipation and "conscious broadcasting." Frontiers in Cognition 3, article 369. (Online journal). doi: 10.3389/fpsyg.2012.00369. [arEM]

Porte, H. S. (2013) From Freud to acetylcholine: Does the AAOM suffice to construct a dream? Behavioral and Brain Sciences 36(6):626-28. [HSP]

Porter, J., Anand, T., Johnson, B., Khan, R. M. \& Sobel, N. (2005) Brain mechanisms for extracting spatial information from smell. Neuron 47:581-92. [BM]

Posner, M. 1. \& Rothbart, M. K. (2000) Developing mechanisms of self-regulation. Development and Psychopathology 12:427-41. [FP]

Postle, B. R. (2009) The hippocampus, memory, and consciousness. In: The neurology of consciousness: Cognitive neuroscience and neuropathology, ed. S. Laureys \& G. Tononi, pp. 326-38. Academic Press. [aEM]

Power, R. P. \& Thompson, W. T. (1970) Simulation of introversion and extraversion on the lemon test. British Journal of Psychology 61:91-93. [AK]

Prechtl, H. F. R. (1993) Principles of early motor development in the human. In: Motor development in early and later childhood: Longitudinal approaches, ed. A. F. Kalverboer, B. Hopkins \& R. Geuze, pp. 35-50. Cambridge University Press. [HD]

Preston, J. \& Wegner, D. M. (2009) Elbow grease: The experience of effort in action. In: Oxford handbook of human action, ed. E. Morsella, J. A. Bargh \& P. M. Gollwitzer, pp. 469-86. Oxford University Press. [rEM]

Preston, S. D. (2013) The origins of altruism in offspring care. Psychological Bulletin 139(6):1305-41. doi: 10.1037/a0031755. [JES]

Price, J. L. (1985) Beyond the primary olfactory cortex: Olfactory-related areas in the neocortex, thalamus, and hypothalamus. Chemical Senses 10:239-85. [aEM]

Price, J. L., Carmichael, S. T., Carnes, K. M., Clugnet, M.-C., Kuroda, M. \& Ray, J. P. (1991) Olfactory input to the prefrontal cortex. In: Olfaction: A model system for computational neuroscience, ed. J. L. Davis \& H. Eichenbaum, pp. 101-20. MIT Press. [aEM]

Prinz, J. (2007) The intermediate level theory of consciousness. In: The Blackwell companion to consciousness, ed. M. Velmans \& S. Schneider, pp. 248-60. Blackwell. [arEM]

Prinz, W. (1984) Modes of linkage between perception and action. In: Cognition and motor processes, ed. W. Prinz \& A. F. Sanders, pp. 185-93. Springer. [WP]

Prinz, W. (2003a) Emerging selves: Representational foundations of subjectivity. Consciousness and Cognition 12:515-28. [WP]

Prinz, W. (2003b) How do we know about our own actions? In: Voluntary action: Brains, minds, and sociality, ed. S. Maasen, W. Prinz \& G. Roth, pp. 21-33. Oxford University Press. [arEM]

Prinz, W. (2012) Open minds: The social making of agency and intentionality. MIT Press. [arEM, WP]

Prinz, W., Aschersleben, G. \& Koch, I. (2009) Cognition and action. In: Oxford handbook of human action, ed. E. Morsella, J. A. Bargh \& P. M. Gollwitzer, pp. 35-71. Oxford University Press. [aEM]

Proctor, G. B. \& Carpenter, G. H. (2007) Regulation of salivary gland function by autonomic nerves. Autonomic Neuroscience 133:3-18. [AK]

Proctor, R. W. \& Vu, K.-P. L. (2010) Action selection. In: The Corsini encyclopedia of psychology, vol. 1, ed. I. B. Weiner \& E. Craighead, pp. 20-22. Wiley. [aEM]

Proffitt, D. R. (2006) Embodied perception and the economy of action. Perspectives on Psychological Science 1(2):110-22. [JSJ]

Puttemans, V., Wenderoth, N. \& Swinnen, S. P. (2005) Changes in brain activation during the acquisition of a multifrequency bimanual coordination task: From the cognitive stage to advanced levels of automaticity. Journal of Neuroscience 25:4270-78. Available at: http://dx.doi.org/10.1523/JNEUROSCI.3866-04.2005 $[\mathrm{rEM}]$

Pylyshyn, Z. W. (1984) Computation and cognition: Toward a foundation for cognitive science. MIT Press. [aEM]

Pylyshyn, Z. W. (1999) Is vision continuous with cognition? The case for cognitive impenetrability of visual perception. Behavioral and Brain Sciences 22:341423. [aEM]

Rakison, D. H. \& Derringer, J. L. (2008) Do infants possess an evolved spiderdetection mechanism? Cognition 107:381-93. [aEM]

Ramachandran, V. S. (1999) Phantoms in the brain: Probing the mysteries of the human mind. Harper Perennial. [rEM]

Raymond, J. E., Shapiro, K. L. \& Arnell, K. M. (1992) Temporary suppression of visual processing in an RSVP task: An attentional blink? Journal of Experimental Psychology: Human Perception and Performance 18:849-60. [PED]

Reder, L. M., ed. (1996) Implicit memory and metacognition. Erlbaum. [BLS] 
Riddle, T. A., Rosen, H. J. \& Morsella, E. (2015) Is that me? Sense of agency as a function of intra-psychic conflict. Journal of Mind and Behavior 36:27-46. [rEM]

Riss, W., Halpern, M. \& Scalia, F. (1969) Anatomical aspects of the evolution of the limbic and olfactory systems and their potential significance for behaviour. Annals of the New York Academy of Sciences 159:1096-111. [RL]

Rizzolatti, G., Fogassi, L. \& Gallese, V. (2004) Cortical mechanisms subserving object grasping, action understanding, and imitation. In: The cognitive neurosciences III, ed. M. S. Gazzaniga, pp. 427-40. MIT Press. [aEM]

Rizzolatti, G., Sinigaglia, C. \& Anderson, F. (2008) Mirrors in the brain: How our minds share actions, emotions, and experience. Oxford University Press. [aEM]

Robertson, L. C. (2003) Binding, spatial attention and perceptual awareness. Nature Reviews: Neuroscience 4:93-102. [aEM]

Robinson, L. (2010) The relationship between perceived physical competence and fundamental motor skills in preschool children. Child: Care, Health and Development 37(4):589-96. [CH]

Rochat, P. (2001) The infant's world. Harvard University Press. [HD]

Roe, A. \& Simpson, G. G. (1958) Behavior and evolution. Yale University Press. [arEM]

Rolls, E. T., Judge, S. J. \& Sanghera, M. (1977) Activity of neurons in the inferotemporal cortex of the alert monkey. Brain Research 130:229-38. [aEM]

Rolls, E. T. \& Treves, A. (1998) Neural networks and brain function. Oxford University Press. [aEM]

Rosenbaum, D. A. (2002) Motor control. In: Stevens' handbook of experimental psychology: Vol. 1. Sensation and perception, 3rd edition, ed. S. Yantis, pp. 31539. [Series editor: H. Pashler]. Wiley. [arEM]

Rosenbaum, D. A. (2005) The Cinderella of psychology: The neglect of motor control in the science of mental life and behavior. American Psychologist 60:308-17. [aEM]

Rosenbaum, D. A. (2010) Human motor control, 2nd edition. Academic Press/ Elsevier. [DAR]

Rosenbaum, D. A. (2014) It's a jungle in there: How competition and cooperation in the brain shape the mind. Oxford University Press. [DAR]

Roser, M. \& Gazzaniga, M. S. (2004) Automatic brains-interpretive minds. Current Directions in Psychological Science 13:56-59. [arEM]

Rossetti, Y. (2001) Implicit perception in action: Short-lived motor representation of space. In: Finding consciousness in the brain: A neurocognitive approach, ed. P. G. Grossenbacher, pp. 133-81. John Benjamins. [aEM]

Russell, J. A. (2003) Core affect and the psychological construction of emotion. Psychological Review 110(1):145-72. Available at: http://doi.org/10.1037/0033295X.110.1.145 [HLM]

Safavi, S., Kapoor, V., Logothetis, N. K. \& Panagiotaropoulos, T. I. (2014) Is the frontal lobe involved in conscious perception? Frontiers in Psychology 5 , article 1063. (Online journal). doi:10.3389/psyg.2014.01063. [aEM]

Saunders, R. C., Mishkin, M. \& Aggleton, J. P. (2005) Projections from the entorhinal cortex, perirhinal cortex, presubiculum, and parasubiculum to the medial thalamus in macaque monkeys: Identifying different pathways using disconnection techniques. Experimental Brain Research 167:1-16. doi: 10.1007/ s00221-005-2361-3. [BM]

Sauseng, P. \& Klimesch, W. (2008) What does phase information of oscillatory brain activity tell us about cognitive processes? Neuroscience and Biobehavioral Reviews 32(5):1001-13. [rEM]

Savic, I., Hedén-Blomqvist, E. \& Berglund, H. (2009) Pheromone signal transduction in humans: What can be learned from olfactory loss. Human Brain Mapping 30:3057-65. doi: 10.1002/hbm.20727. [BM]

Saxton, T. K., Lyndon, A., Little, A. C \& Roberts, S. C. (2008) Evidence that androstadienone, a putative human chemosignal, modulates women's attributions of men's attractiveness. Hormones and Behavior 54:597-601. [BM]

Schachter, S. \& Singer, J. E. (1962) Cognitive, social, and physiological determinants of emotional state. Psychological Review. 69:379-99. [AKS]

Schachter, D. L. (1987) Implicit memory: History and current status. Journal of Experimental Psychology 13:501-18. [FP]

Schacter, D. L. (1996) Searching for memory: The brain, the mind, and the past. Basic Books. [aEM]

Schaffer, M. K.-H., Eiden, L. E. \& Weihe, E. (1998) Cholinergic neurons and terminal fields revealed by immunohistochemistry for the vesicular acetylcholine transporter: I. Central nervous system. Neuroscience 84:331-59. [HSP]

Schelling, F. (1800/1978) System of transcendental philosophy, trans. P. Heath. University Press of Virginia. (Original work published in 1800). [FP]

Schlaghecken, F., Bowman, H. \& Eimer, M. (2006) Dissociating local and global levels of perceptuo-motor control in masked priming. Journal of Experimental Psychology: Human Perception and Performance 32(3):618-32. [AA, rEM]

Schlaghecken, F. \& Eimer, M. (2006) Active masks and active inhibition: A comment on Lleras and Enns (2004) and on Verleger, Jaśkowski, Aydemir, van der Lubbe, and Groen (2004). Journal of Experimental Psychology: General 135 (3):484-94. [AA]

Schmahmann, J. D. (1998) Dysmetria of thought: Clinical consequences of cerebellar dysfunction on cognition and affect. Trends in Cognitive Sciences 2:36271. [aEM]
Schmahmann, J. D. (2001) The cerebrocerebellar system: Anatomic substrates of the cerebellar contribution to cognition and emotion. International Review of Psychiatry 13(4):247-60. [JSJ]

Schoeller, F. (2015) The shivers of knowledge. Human and Social Studies 4(3):26-41. ISSN (Online) 2285-5920, DOI: 10.1515/hssr-2015-0022, November 2015. [LP]

Schoeller, F. \& Perlovsky, L. (2015) Great expectations - Narratives and the elicitation of aesthetic chills. Psychology 6(16):2098-102; doi: 10.4236/ psych.2015.616205. Available at: http://www.scirp.org/journal/psych [LP]

Schoenbaum, G. \& Eichenbaum, H. (1995) Information coding in the rodent prefrontal cortex: I. Single-neuron activity in orbitofrontal cortex compared with that in pyriform cortex. Journal of Neurophysiology 74:733-50. [aEM]
[a.

Scholl, B. J. (2001) Objects and attention: The state of the art. Cognition 80:9-17. [aEM]

Schopenhauer, A. (1818/1819) The world as will and representation, vol. 1. Dover. (Original work published in 1818). [aEM]

Schroter, M., Spoormaker, V., Schorer, A., Wohlschlager, A., Czish, M., Kochs, E., Zimmer, C., Hemmer, B., Schneider, G., Jordan, D. \& Ilg, R. (2012) Spatiotemporal reconfiguration of large-scale brain functional networks during propofolinduced loss of consciousness. Journal of Neuroscience 32:12832-40. [arEM]

Schrouff, J., Perlbarg, V., Boly, M., Marrelec, G., Boveroux, P., Vanhaudenhuyse, A., Bruno, M. A., Laureys, S., Phillips, C., Pélégrini-Isaac, M., Maquet, P. \& Benali, H. (2011) Brain functional integration decreases during propofol-induced loss of consciousness. NeuroImage 57:198-205. [arEM]

Schubert, R., Blankenburg, F., Lemm, S., Villringer, A. \& Curio, G. (2006) Now you feel it - now you don't: ERP correlates of somatosensory awareness. Psychophysiology 43:31-40. [aEM]

Schurger, A., Sarigiannidis, I., Naccache, L., Sitt, J. D. \& Dehaene, S. (2015) Cortical activity is more stable when sensory stimuli are consciously perceived. Proceedings of the National Academy of Sciences USA 112(16):E2083-92. [LM]

Schwartz, B. L. \& Cleary, A. M. (2016) Tip-of-the-tongue states, déjà vu and other metacognitive oddities. In: Oxford handbook of metamemory, ed. J. Dunlosky \& S. Tauber, pp. 95-108. Oxford University Press. [BLS]

Schwartz, B. L. \& Metcalfe, J. (2011) Tip-of-the-tongue (TOT) states: Retrieval, behavior, and experience. Memory and Cognition 39:737-49. [BLS]

Scott, M. (2013) Corollary discharge provides the sensory content of inner speech. Psychological Science 24:1824-30. [aEM]

Searle, J. R. (2000) Consciousness. Annual Review of Neurosciences 23:557-78. [arEM]

Sela, L., Sacher, Y., Serfaty, C., Yeshurun, Y., Soroker, N. \& Sobel, N. (2009) Spared and impaired olfactory abilities after thalamic lesions. Journal of Neuroscience 29:12059-69. [aEM]

Sela, L. \& Sobel, N. (2010) Human olfaction: A constant state of change-blindness. Experimental Brain Research 205:13-29. doi: 10.1007/s00221-010-2348-6. [BM]

Selfridge, O. G. (1959) Pandemonium: A paradigm for learning. In: Mechanization of thought processes: Proceedings of a Symposium held at the National Physics Laboratory, November 1958, vol. 1, ed. D. V. Blake \& A. J. Uttley. pp. 511-26. Her Majesty's Stationary Office. [rEM]

Sergent, C. \& Dahaene, S. (2004) Is consciousness a gradual phenomenon? Evidence for an all-or-none bifurcation during the attentional blink. Psychological Science 15:720-28. [arEM]

Seth, A. K. (2007) The functional utility of consciousness depends on content as well as on state. Behavioral and Brain Sciences 30(1):106. [aEM]

Seth, A. K. (2013) Interoceptive inference, emotion, and the embodied self. Trends in Cognitive Sciences 17(11):565-73. doi: 10.1016/j.tics.2013.09.007. [AKS]

Seth, A. K. (2015) The cybernetic bayesian brain: From interoceptive inference to sensorimotor contingencies. In: Open MIND, ed. T. K. Metzinger \& J. M. Windt, pp. 1-24. MIND Group. [AKS]

Seth, A. K., Verschure, P., Blanke, O., Butz, M., Ford, J. M., Frith, C. D., Jacob, P., Kyselo, M., McGann, M., Menary, M., Morsella, E. \& O’Regan, J. K. (2016) Action-oriented understanding of consciousness and the structure of experience. In: The pragmatic turn: Toward action-oriented views in cognitive science, ed. A. K. Engel, K. J. Friston, \& D. Kragic, pp. 261-285. [Strüngmann Forum Reports, vol. 18; J. Lupp, series editor]. MIT Press. [AKS]

Shallice, T. (1972) Dual functions of consciousness. Psychological Review 79:38393. $[\mathrm{rEM}]$

Shariff, A. F., Schooler, J. \& Vohs, K. D. (2008) The hazards of claiming to have solved the hard problem of free will. In: Are we free? Psychology and free will, ed. J. Baer, J. C. Kaufman \& R. F. Baumeister, pp. 181-204. Oxford University Press. $[\mathrm{BH}]$

Sheerer, E. (1984) Motor theories of cognitive structure: A historical review. In Cognition and motor processes, ed. W. Prinz \& A. F. Sanders, pp. 77-98. Springer-Verlag. [aEM]

Shen, Y. C. \& Franz, E. A. (2005) Hemispheric competition in left-handers on bimanual reaction time tasks. Journal of Motor Behavior 37:3-9. [EAF]

Shepherd, G. M. (2006) Smell images and the flavour system in the human brain. Nature 444(7117):316-21. [rEM]

Shepherd, G. M. (2007) Perspectives on olfactory processing, conscious perception, and orbitofrontal cortex. Annals of the New York Academy of Sciences 1121:87101. [arEM, RL] 
References/Morsella et al.: Homing in on consciousness in the nervous system

Shepherd, G. M. \& Greer, C. A. (1998) Olfactory bulb. In: The synaptic organization of the brain, 4th edition, ed. G. M. Shepherd, pp. 159-204. Oxford University Press. [aEM]

Sherman, S. M. \& Guillery, R. W. (2006) Exploring the thalamus and its role in cortical function. MIT Press. [aEM]

Sherrington, C. S. (1906) The integrative action of the nervous system. Yale University Press. [aEM]

Siegel, M., Donner, T. H. \& Engel, A. K. (2012) Spectral fingerprints of large-scale neuronal interactions. Nature Reviews Neuroscience 13:121-34. [rEM]

Simons, D. J. \& Levin, D. T. (1997) Change blindness. Trends in Cognitive Sciences 1:261-67. [rEM]

Simpson, G. G. (1949) The meaning of evolution. Yale University Press. [arEM]

Singer, W. (2011) Consciousness and neuronal synchronization. In: The neurology of consciousness, ed. S. Laureys \& G. Tononi, pp. 43-52. Academic Press. [arEM]

Skinner, B. F. (1953) Science and human behavior. Macmillan. [arEM]

Slevc, L. R. \& Ferreira, V. S. (2006) Halting in single word production: A test of the perceptual loop theory of speech monitoring. Journal of Memory and Language 54:515-40. [aEM]

Slotnick, B. M. \& Risser, J. M. (1990) Odor memory and odor learning in rats with lesions of the lateral olfactory tract and mediodorsal thalamic nucleus. Brain Research 529:23-29. [aEM]

Smith, D. \& Over, R. (1987) Male sexual arousal as a function of the content and the vividness of erotic fantasy. Psychophysiology 24(3):334-39. [AK]

Smith, P. K., Bowers, L., Binney, V. \& Cowie, H. (1993) Relationships of children involved in bully/victim problems at school. In: Learning about relationships. Understanding Relationship Processes Series, vol. 2, ed. S. Duck, pp. 184-215. Sage. $[\mathrm{FP}]$

Sobel, N., Prabhakaran, V., Hartley, C. A., Desmond, J. E., Glover, G. H., Sullivan E. V. \& Gabrieli, J. D. E. (1999) Blind smell: Brain activation induced by an undetected airborne chemical. Brain 122:209-17. [JdV]

Sobel, N., Prabhakaran, V., Zhao, Z., Desmond, J. E., Glover, G. H., Sullivan, E. V. \& Gabrieli, J. D. (2000) Time course of odorant-induced activation in the human primary olfactory cortex. Journal of Neurophysiology 83(1):537-51. [rEM]

Sokolov, E. N. (1963) Higher nervous functions: The orienting reflex. Annual Review of Physiology 25:545-80. doi:10.1146/annurev.ph.25.030163.002553. [BM]

Soon, C. S., Brass, M., Heinze, H.-J. \& Haynes, J. D. (2008) Unconscious determinants of free decisions in the human brain. Nature Neuroscience 11:543-45. [RL]

Spear, N. E. \& Campbell, B. A. (1979) Ontogeny of learning and memory. Erlbaum. [aEM]

Spence, C. (2011) Mouth-watering: The influence of environmental and cognitive factors on salivation and gustatory/flavor perception. Journal of Texture Studies 42:157-71. [AK]

Sperry, R. W. (1952) Neurology and the mind-brain problem. American Scientist 40:291-312. [arEM, EAF]

Spivey, M. (2008) The continuity of mind. Oxford University Press. [HLM]

Squire, L. R. (1987) Memory and brain. Oxford University Press. [aEM]

Squire, L. R. (2004) Memory systems of the brain: A brief history and current perspective. Neurobiology of Learning and Memory 82(3):171-77. [MGa]

Squire, L. R., Wixted, J. T. \& Clark, R. E. (2007) Recognition memory and the medial temporal lobe: A new perspective. Nature Reviews in Neuroscience 8 (11):872-83. [MGa]

Srinivasan, R., Russell, D. P., Edelman, G. M. \& Tononi, G. (1999) Increased synchronization of neuromagnetic responses during conscious perception. The Journal of Neuroscience 19:5435-48. [aEM]

Srull, T. K. (1997) The vicissitudes of social behavior and mental life. In: The automaticity of everyday life: Advances in social cognition, vol. 10, ed. R. S. Wyer Jr., pp. 203-16. Erlbaum. [FP]

Stefanucci, J. K. \& Geuss, M. N. (2009) Big people, little world: The body influences size perception. Perception 38:1782-95. [aEM]

Stepp, N. \& Turvey, M. T. (2015) The muddle of anticipation. Ecological Psychology 27(2):103. doi:10.1080/10407413.2015.1027123. [JdV]

Stevenson, R. J. (2009) Phenomenal and access consciousness in olfaction. Consciousness and Cognition 18:1004-17. doi:10.1016/j.concog.2009.09.005. [arEM, BM]

Stopfer, M., Bhagavan, S., Smith, B. H. \& Laurent, G. (1997) Impaired odour discrimination on desynchronization of odour-encoding neural assemblies. Nature 390(6655):70-74. [rEM]

Stroop, J. R. (1935) Studies of interference in serial verbal reactions. Journal of Experimental Psychology 18:643-62. [aEM]

Stuss, D. T. \& Anderson, V. (2004) The frontal lobes and theory of mind: Developmental concepts from adult focal lesion research. Brain \& Cognition 55:69-83. [aEM]

Suhler, C. L. \& Churchland, P. S. (2009) Control: Conscious and otherwise. Trends in Cognitive Sciences 13:341-47. [arEM]

Sumner, P., Nachev, P., Morris, P., Peters, A. M., Jackson, S. R., Kennard, C. \& Husain, M. (2007) Human medial frontal cortex mediates unconscious inhibition of voluntary action. Neuron 54(5):697-711. [AA]

Sutton, J. \& Smith, P. K. (1999) Bullying as a group process: An adaptation of the participant role approach. Aggressive Behavior 25:97-111. [FP]
Suzuki, T., Itoh, S., Arai, N., Kouno, M., Noguchi, M., Takatsu, M. \& Takeda, K. (2012) Ambient echolalia in a patient with germinoma around the bilateral ventriculus lateralis: A case report. Neurocase 18:330-35. [aEM]

Swain, J. E. (2007) Critical developmental periods of increased plasticity program ritualized behavior. Behavioral and Brain Sciences 29(3):242-43. [JES]

Swain, J. E., Dayton, C. J., Kim, P., Tolman, R. M. \& Volling, B. L. (2014a) Progress on the paternal brain: Theory, animal models, human brain research, and mental health implications. Infant Mental Health Journal 35(5):394-408. doi: 10.1002/imhj.21471. [JES]

Swain, J. E., Kim, P., Spicer, J., Ho, S. S., Dayton, C. J., Elmadih, A. \& Abel, K. M (2014b) Approaching the biology of human parental attachment: Brain imaging, oxytocin and coordinated assessments of mothers and fathers. Brain Research 1580:78-101. doi: 10.1016/j.brainres.2014.03.007. [JES]

Swain, J. E., Konrath, S., Brown, S. L., Finegood, E. D., Akce, L. B., Dayton, C. J. \& Ho, S. S. (2012) Parenting and beyond: Common neurocircuits underlying parental and altruistic caregiving. Parenting, Science and Practice 12(2-3):11523. doi: 10.1080/15295192.2012.680409. [JES]

Swain, J. E., Mayes, L. C. \& Leckman, J. F. (2004) The development of parent-infant attachment through dynamic and interactive signaling loops of care and cry. Behavioral and Brain Sciences 27(4):472-73. [JES]

Swenson, R. (1997) Evolutionary theory developing: The problem(s) with Darwin's dangerous idea. Ecological Psychology 9(1):47-96. [CH]

Synofzik, M., Vosgerau, G. \& Newen, A. (2008) I move, therefore I am: A new theoretical framework to investigate agency and ownership. Consciousness and Cognition 17:411-24. [aEM]

Tallon-Baudry, C. (2012) On the neural mechanisms subserving attention and consciousness. Frontiers in Psychology 2, article 397. (Online journal). doi: 10.3389/ fpsyg.2011.00397. [aEM]

Tanaka, Y., Miyazawa, Y., Akaoka, F. \& Yamada, T. (1997) Amnesia following damage to the mammillary bodies. Neurology 48:160-65. [aEM]

Tassinari, G. \& Berlucchi, G. (1993) Sensory and attentional components of slowing of manual reaction time to non-fixated visual targets by ipsilateral primes. Vision Research 33(11):1525-34. [EAF]

Taylor, J. A. \& Ivry, R. B. (2013) Implicit and explicit processes in motor learning. In: Action science, ed. W. Prinz, M. Beisert \& A. Herwig, pp. 63-87. MIT Press. [aEM]

Taylor, J. L. \& McCloskey, D. I. (1990) Triggering of preprogrammed movements as reactions to masked stimuli. Journal of Neurophysiology 63:439-46. [aEM]

Taylor, J. L. \& McCloskey, D. I. (1996) Selection of motor responses on the basis of unperceived stimuli. Experimental Brain Research 110:62-66. [aEM]

Tetlock, P. E. (2002) Social functionalist frameworks for judgment and choice: Intuitive politicians, theologians, and prosecutors. Psychological Review 109:45171. [rEM]

Thagard, P. \& Stewart, T. C. (2014) Two theories of consciousness: Semantic pointer competition vs. information integration. Consciousness and Cognition 30:7390. [ arEM]

Tham, W. W. P., Stevenson, R. J. \& Miller, L. A. (2009) The functional role of the medio dorsal thalamic nucleus in olfaction. Brain Research Reviews 62:109 26. [aEM]

Tham, W. W. P., Stevenson, R. J. \& Miller, L. A. (2011) The role of the mediodorsal thalamic nucleus in human olfaction. Neurocase 17:148-59. [aEM]

Thelen, E. \& Smith, L. B. (1996) A dynamic systems approach to the development of cognition and action. MIT Press. [HD]

Thompson, E. \& Varela, F. J. (2001) Radical embodiment: Neural dynamics and consciousness. Trends in Cognitive Sciences 5(10):418-25. [CH]

Thorndike, E. L. (1905) The functions of mental states. In: The elements of psychology, ed. E. L. Thorndike, pp. 111-19. A. G. Seiler. [rEM]

Tipper, S. P. (1985) The negative priming effect: Inhibitory priming by ignored objects. The Quarterly Journal of Experimental Psychology 37A:571-90. [rEM]

Todorov, A. \& Bargh, J. A. (2002) Automatic sources of aggression. Aggression and Violent Behavior 7:53-68. [FP]

Tolman, E. C. (1948) Cognitive maps in rats and men. Psychological Review 55:189208. [JdV, rEM]

Tong, F. (2003) Primary visual cortex and visual awareness. Nature Reviews Neuroscience 4:219-29. [aEM]

Tononi, G. (2012) Phi: A voyage from the brain to the soul. Pantheon. [aEM]

Tononi, G. (2013) Integrated information theory of consciousness: An updated account. Archives Italiennes de Biologie 150(2-3):290-326. [LM]

Tononi, G. \& Edelman, G. M. (1998) Consciousness and complexity. Science 282 (5395):1846-51. [aEM, LM]

Tranel, D. \& Welsh-Bohmer, K. A. (2012) Pervasive olfactory impairment after bilateral limbic system destruction. Journal of Clinical and Experimental Neuropsychology 34:117-25. [aEM]

Treisman, A. M. (2003) Consciousness and perceptual binding. In: The unity of consciousness: Binding, integration, and dissociation, ed. A. Cleeremans, pp. 95-113. Oxford University Press. [LM]

Tsotsos, J. K. (1995) Behaviorist intelligence and the scaling problem. Artificial Intelligence 75:135-60. [rEM] 
Tsotsos, J. K. (2011) A computational perspective on visual attention. MIT Press. $[\mathrm{rEM}]$

Tsushima, Y., Sasaki, Y. \& Watanabe, T. (2006) Greater disruption due to failure of inhibitory control on an ambiguous distractor. Science 314:1786-88. [aEM]

Tucker, M. \& Ellis, R. (2004) Action priming by briefly presented objects. Acta Psychologica 116(2):185-203. [AA]

Turvey, M. T. (2015) Quantum-like issues at nature's ecological scale (the scale of organisms and their environments). Mind and Matter 13:7-44. [JdV]

Tye, M. (1999) Phenomenal consciousness: The explanatory gap as cognitive illusion. Mind 108:705-25. [aEM]

Uhlhaas, P. J., Pipa, G., Lima, B., Melloni, L., Neuenschwander, S., Nikolic, D. \& Singer, W. (2009) Neural synchrony in cortical networks: History, concept and current status. Frontiers in Integrative Neuroscience 3, article 17. (Online journal). doi:10.3389/neuro.07.017.2009. [arEM]

Vallacher, R. R. \& Wegner, D. M. (1987) What do people think they're doing? Action identification and human behavior. Psychological Review 94:3-15. [DAR]

van Gaal, S., Ridderinkhof, K. R., Fahrenfort, J. J., Scholte, H. S. \& Lamme, V. A. F. (2008) Frontal cortex mediates unconsciously triggered inhibitory control. Journal of Neuroscience 28:8053-62. [aEM]

Van Opstal, F., Gevers, W., Osman, M. \& Verguts, T. (2010) Unconscious task application. Consciousness and Cognition 19:999-1006. [rEM]

van Veen, V., Cohen, J. D., Botvinick, M. M., Stenger, V. A. \& Carter, C. C. (2001) Anterior cingulate cortex, conflict monitoring, and levels of processing. Neuroimage 14:1302-308. [aEM]

Vanderwolf, C. H. \& Zibrowski, E. M. (2001) Pyriform cortex beta-waves: Odorspecific sensitization following repeated olfactory stimulation. Brain Research 892:301-308. [rEM]

Varela, F., Lachaux, J. P., Rodriguez, E. \& Martinerie, J. (2001) The brainweb: Phase synchronization and large-scale integration. National Review of Neuroscience 2:229-39. [aEM]

Velly, L., Rey, M., Bruder, N., Gouvitsos, F., Witjas, T., Regis, J. M., Peragut, J. C. \& Gouin, F. (2007) Differential dynamic of action on cortical and subcortical structures of anesthetic agents during induction of anesthesia. Anesthesiology 107:202-12. [aEM]

Velmans, M. (1991) Is human information processing conscious? Behavioral and Brain Sciences 14(4):651-69. [aEM]

Vierkant, T. (2013) Managerial control and free mental agency. In: Decomposing the will, ed. A. Clark, J. Kiverstein \& T. Vierkant, pp. 285-97. Oxford University Press. [aEM]

Vinson, D. W., Jordan, J. S. \& Hund, A. M. (2015) Perceptually walking in another's shoes: Goals and memories constrain spatial perception. Psychological Research 79(5):1-9. doi:10.1007/s00426-015-0714-5. [JSJ]

Vishton, P. M., Stephens, N. J., Nelson, L. A., Morra, S. E., Brunick, K. L. \& Stevens, J. A. (2007) Planning to reach for an object changes how the reacher perceives it. Psychological Science 18(8):713-19. doi: 10.1111/j.14679280.2007.01965.x. [AKS]

von Hofsten, C. (2007) Action in development. Developmental Science 10:54-60. [HD]

von Stein, A. \& Sarnthein, J. (2000) Different frequencies for different scales of cortical integration: From local gamma to long range alpha/theta synchronization. International Journal of Psychophysiology 38(3):301-13. [rEM]

Voss, M. (2011) Not the mystery it used to be: Theme program: Consciousness. APS Observer 24(6), July/August 2011 edition. Available at: http://www. psychologicalscience.org [aEM]

Vroomen, J. \& de Gelder, B. (2003) Visual motion influences the contingent auditory motion aftereffect. Psychological Science 14:357-61. [aEM]

Wachowski, A. \& Wachowski, L. (1999) The Matrix. Warner Bros. [BLS]

Ward, L. M. (2003) Synchronous neural oscillations and cognitive processes. Trends in Cognitive Sciences 7:553-59. [rEM]

Ward, L. M. (2011) The thalamic dynamic core theory of conscious experience. Consciousness and Cognition 20:464-86. [aEM]

Wegner, D. M. (2002) The illusion of conscious will. MIT Press. [aEM]

Wegner, D. M. \& Bargh, J. A. (1998) Control and automaticity in social life. In: Handbook of social psychology, 4th edition, ed. D. Gilbert, S. T. Fiske \& G. Lindzey, pp. 446-96. McGraw-Hill. [FP]

Weiskrantz, L. (1992) Unconscious vision: The strange phenomenon of blindsight The Sciences 35:23-28. [aEM]

Weiskrantz, L. (1997) Consciousness lost and found: A neuropsychological exploration. Oxford University Press. [aEM]

Welford, A. T. (1952) The "psychological refractory period" and the timing of highspeed performance: A review and a theory. British Journal of Psychology 43:219. $[\mathrm{rEM}]$

Werner, G. (2007) Metastability, criticality and phase transitions in brain and its models. Biosystems 90(2):496-508. doi:10.1016/j.biosystems.2006.12.001. [CH]

Werner, H. \& Kaplan, B. (1963) Symbol formation. Wiley. [rEM]

Wessel, J. R., Haider, H. \& Rose, M. (2012) The transition from implicit to explicit representations in incidental learning situations: More evidence from highfrequency EEG coupling. Experimental Brain Research 217:153-62. [arEM]
Westwood, D. A. (2009) The visual control of object manipulation. In: Oxford handbook of human action, ed. E. Morsella, J. A. Bargh \& P. M. Gollwitzer, pp 88-103. Oxford University Press. [aEM]

White, K. D. (1978) Salivation: The significance of imagery in its voluntary control. Psychophysiology 15:196-203. [AK]

Wiers, R. W., Field, M. \& Stacy, A. W. (2014) Passion's slave? Cognitive processes in alcohol and drug abuse. In: Oxford handbook of substance use and substance use disorders, vol. 1, ed. K. J. Sher. Oxford Handbooks Online/Oxford University Press. doi:10.1093/oxfordhb/9780199381678.013.009. [Published Online, July 2014. Available at: http://www.oxfordhandbooks.com/view/ 10.1093/oxfordhb/9780199381678.001.0001/oxfordhb-9780199381678]. [BH]

Wilke, M., Mueller, K.-M. \& Leopold, D. A. (2009) Neural activity in the visual thalamus reflects perceptual suppression. Proceedings of the National Academy of Sciences USA 106:9465-70. doi:10.1073/pnas.0900714106. [BM, rEM]

Withagen, R. \& van Wermeskerken, M. (2010) The role of affordances in the evolutionary process reconsidered: A niche construction perspective. Theory and Psychology 20:489-510. [JdV]

Wolford, G., Miller, M. B. \& Gazzaniga, M. S. (2004) Split decisions. In: The cognitive neurosciences III, ed. M. S. Gazzaniga, pp. 1189-99. MIT Press. [aEM]

Wong, R. O. L. (1999) Retinal waves and visual system development. Annual Review of Neuroscience 22:29-47. [HSP]

Woodman, G. F. \& Vogel, E. K. (2005) Fractionating working memory: Consolidation and maintenance are independent. Psychological Science 16:106-113. [rEM]

Woodworth, R. S. (1915) A revision of imageless thought. Psychological Review 22:1-27. [aEM]

Wundt, W. (1900) Die sprache. Engelmann. [raEM]

Wundt, W. (1902/1904) Principles of physiological psychology, trans. E. B. Titchener. Sonnenschein. (Original work published in 1902; English translation from the 5th German edition [1904]). [arEM]

Wyart, C., Webster, W. W., Chen, J. H., Wilson, S. R., McClary, A., Khan, R. M. \& Sobel, N. (2007) Smelling a single component of male sweat alters levels of cortisol in women. Journal of Neuroscience 27:1261-65. doi:10.1523/JNEUROSCI.4430-06.2007. [BM]

Wysocki, C. J. \& Preti G. (2004) Facts, fallacies, fears, and frustrations with human pheromones. Anatomical Record (Part A) 281A:1201-11. [BM]

Xu, F., Greer, C. A. \& Shepherd, G. M. (2000) Odor maps in the olfactory bulb Journal of Comparative Neurology 422(4):489-95. [rEM]

Yamadori, A. (1997) Body awareness and its disorders. In: Cognition, computation and consciousness, ed. M. Ito, Y. Miyashita \& E. T. Rolls, pp. 169-76. American Psychological Association. [aEM]

Yamamoto, T. \& Yasoshima, Y. (2007) Electrophysiological representation of taste memory. In: Neural plasticity and memory: From genes to brain imaging, ed. F. Bermudez-Rattoni, pp. 113-28. CRC Press. [MGa]

Yates, J. (1985) The content of awareness is a model of the world. Psychological Review 92:249-84. [aEM]

Yeshurun, Y. \& Sobel, N. (2010) An odor is not worth a thousand words: From multidimensional odors to unidimensional odor objects. Annual Review of Psychology 61:219-41. doi:10.1146/annurev.psych.60.110707.163639. [BM]

Zatorre, R. J. \& Jones-Gotman, M. (1991) Human olfactory discrimination after unilateral frontal or temporal lobectomy. Brain 114:71-84. [aEM]

Zeki, S. \& Bartels, A. (1999) Toward a theory of visual consciousness. Consciousness and Cognition 8:225-59. [aEM]

Zelazo, P. D. (2004) The development of conscious control in childhood. Trends in Cognitive Sciences 8:12-17. [HDS]

Zelli, A., Cervone, D. \& Huesmann, L. R. (1996) Behavioral experience and social inference: Individual differences in aggressive experience and spontaneous versus deliberate trait inference. Social Cognition 14:165-90. [FP]

Zelli, A., Huesmann, L. R. \& Cervone, D. (1995) Social inference and individual differences in aggression: Evidence for spontaneous judgements of hostility. Aggressive Behavior 21:405-17. [FP]

Zhang, P., Jamison, K., Engel, S., He, B. \& He, S. (2011) Binocular rivalry requires visual attention. Neuron 71:362-69. [PED]

Zhang, W. \& Rosenbaum, D. A. (2008) Planning for manual positioning: The endstate comfort effect for abduction-adduction of the hand. Experimental Brain Research 184:383-89. [aEM]

Zhou, W. \& Chen, D. (2009) Fear-related chemosignals modulate recognition of fear in ambiguous facial expressions. Psychological Science 20:177-83. doi: 10.1111/ j.1467-9280.2009.02263.x. $\quad[\mathrm{BM}]$

Zhu, Q. \& Bingham, G. (2010) Learning to perceive the affordance for long-distance throwing: Smart mechanism or function learning? Journal of Experimental Psychology: Human Perception and Performance 36(4):862-75. [CH]

Zibrowski, E. M. \& Vanderwolf, C. H. (1997) Oscillatory fast wave activity in the rat pyriform cortex: Relations to olfaction and behavior. Brain Research 766:3949. [rEM]

Zucco, G. M., Priftis, K. \& Stevenson, R. J. (2014) From blindsight to blindsmell: A mini review. Translational Neuroscience 6:8-12. [JdV] 